\title{
1st International Congress on Clinical Neurology and Epidemiology
}

Munich, Germany, August 27-30, 2009

\section{Abstracts}

\section{Editors}

V.L. Feigin, Auckland, New Zealand

M.G. Hennerici, Mannheim, Germany

Extended abstracts of Plenary and Teaching Sessions as well as Round Table will be published in fall 2009 in Neuroepidemiology. 
Programme Committee

\section{Chairman}

Feigin, Valery L., New Zealand

Members

Barnes, Michael, UK

Hennerici, Michael G., Germany

Homma, Akira , Japan

Rocca, Walter, USA

Tuomilehto, Jaakko, Finland

\section{Organising Committee}

\section{Chairman}

Hennerici, Michael G., Germany

\section{Members}

Amarenco, Pierre, France

Bäzner, Hansjörg, Germany

Diener, Hans C., Germany

Feigin, Valery L., New Zealand

Hartung, Hans-Peter, Germany

Oertel, Wolfgang, Germany

Orgogozo, Jean-Marc, France

Rothwell, Peter, UK

\section{About the Congress}

The First International Congress on Clinical Neurology and Epidemiology (Neuroepidemiology) scheduled to take place in Munich, Germany in August 27-30, 2009 is a unique congress for many reasons.

Neuroepidemiology has been perceived for a long time as a science of incidence, prevalence, risk factors, natural history and prognosis of neurological disorders. However, it is only one part of neuroepidemiology called nonexperimental neuroepidemiology. The other integral, but commonly forgotten, part of neuroepidemiology is an experimental neuroepidemiology, a research based on clinical trials of effectiveness or efficacy of various interventions in neurological disorders.

This International Congress, for the first time, will bring together scientists and experts in all major fields of experimental and non-experimental neuroepidemiology.

The Congress will feature internationally recognized invited speakers, platform lectures, oral presentations and poster sessions, and will provide an ideal platform for continuing education in all fields of experimental and nonexperimental clinical neuroepidemiology. 


\section{Contents}

\section{Oral Presentations}

SS1 - Stroke Epidemiology and Management (Part 1)

SS2 - Parkinson's Disease and Other Movement Disorders - Epidemiology and Management (Part 1)

SS3 - Multiple Sclerosis Epidemiology

SS4 - Epidemiology of Traumatic Brain Injury

SS5 - Migraine and Other Pain Disorders Epidemiology

SS6 - Stroke Epidemiology and Management (Part 2)

SS7 - Epilepsy and Other Seizure/Neurological Disorders Epidemiology (Part 1)

SS8 - Stroke Epidemiology and Management (Part 3)

SS9 - Epilepsy and Other Seizure/Neurological Disorders Epidemiology (Part 2)

SS10 - Parkinson's Disease and Other Movement Disorders - Epidemiology and Management (Part 2)

SS1 1 - Stroke Epidemiology and Management (Part 4)

SS12 - Motor Neuron and Parkinson's Disease Epidemiology

SS13 - Stroke Epidemiology and Management (Part 5)

SS14 - Epidemiology of CNS Tumours and Infections

SS15 - Stroke Epidemiology and Management (Part 6)

SS16 - Translational Neurogenetic Neuroepidemiology Research

SS17 - Epidemiology of Dementia and Other Cognitive Disorders

SS18 - Stroke Epidemiology and Management (Part 7)

SS19 - Other Neurological Conditions / Issues

\section{Poster Presentations}

P2 - Epidemiology of CNS Tumours and Infections

P3 - Epidemiology of Dementia and Other Cognitive Disorders

P4 - Developmental Disorders of the Nervous System

P5 - Epilepsy and Other Seizure Disorders Epidemiology

P6 - Migraine and Other Pain Disorders Epidemiology

P8 - Multiple Sclerosis and Other Demyelinative

P9 - Neurological Aspects of Aging

P13 - Neuromuscular Disorders

P15 - Peripheral Neuropathy

P18 - Traumatic Brain Injury

P19 - Other Neurological Disorders 


\section{SS1 \\ Stroke Epidemiology and Management (Part 1)}

August 28, 2009

09.00-10.00

\section{SS1/1 \\ A Clinical Epidemiology Perspective on the Dissemination of Stroke Thrombolysis Across Sweden}

Stegmayr B. ${ }^{1}$, Jonsson F.2 Appelros P. ${ }^{3}$, Hulter Asberg K. ${ }^{4}$ Norrving B. ${ }^{5}$, Terént A. ${ }^{6}$, Asplund $K^{2}{ }^{2}$, The Riks-Stroke Collaboration

${ }^{1}$ National Board of Health and Welfare, Stockholm, Sweden, ${ }^{2}$ Department of Medicine, University Hospital, Riks-Stroke, Umea, Sweden, ${ }^{3}$ Department of Neurology, University Hospital, Orebro, Sweden, ${ }^{4}$ Department of Medicine, Enkoping Hospital, Enkoping, Sweden, ${ }^{5}$ Department of Neurology, University Hospital, Lund, Sweden, ${ }^{6}$ Department of Medicine, Akademiska University Hospital, Uppsala, Sweden

Background: Improved knowledge about how new techniques are disseminated in stroke care may help to improve rapid nationwide implementation of novel interventions. We have explored how thrombolysis for acute ischemic stroke has been disseminated across Sweden.

Methods: The Riks-Stroke register covers all hospitals in Sweden admitting acute stroke patients. During the years 200108 , more than 90000 patients $<==80$ years with acute ischemic stroke were registered.

Results: After the approval by the national regulatory authority in 2003, the uptake of thrombolysis increased from $0.9 \%$ in 2003 to $5.4 \%$ in 2007 and $6.3 \%$ in 2008 (preliminary). Dissemination of thrombolysis occurred at a similar pace in men and women. Median age of patients receiving thrombolytic therapy increased slightly from 66 years in 2001-02 to 68 years in 2007 . There were marked regional differences in the implementation of thrombolysis but, as of 2008, patients in all 21 counties in the country have access to thrombolysis. A similar proportion of patients receiving thrombolysis was reached about 2 years later in community hospitals compared to university hospitals. University, intermediate and community hospitals had similar proportions of patients with early improvement following thrombolysis and similar frequencies of intracerebral hemorrhage with clinical deterioration within 36 hours.
Conclusions: Nationwide implementation of thrombolysis has been slow but it has accelerated in the last few year. The implementation can be performed with preserved patient safety but it has been fragmented with considerable regional inequity in the access to this new intervention.
SS1/2

\section{Long-Term Exposure to Air Pollution and Ischemic Stroke: A Case-Control Study in Southern Sweden}

\author{
Oudin A. ', Stroh E. ', Strömberg U. ${ }^{1}$, Björk J. ${ }^{2}$, \\ Jakobsson $K^{3}$ \\ ${ }^{1}$ Department for Occupational and Environmental \\ Medicine, Lund University, Lund, Sweden, ${ }^{2}$ Competence \\ Centre for Clinical Research, Lund, Sweden, ${ }^{3}$ Lund \\ University Hospital, Lund, Sweden
}

Background: Long-term exposure to air pollution is a suggested risk factor for ischemic stroke. We investigated such an association in the region of Scania in Southern Sweden, where levels of air pollution are generally low.

Methods: We used a two-phase case-control study design. First-phase cases were defined as all Scanian first-time ischemic stroke patients registered in the Swedish stroke register 20012005 and born 1923-1965 ( $\mathrm{N}=4$ 904). As first-phase controls we selected all individuals born 1923-1965 and residing in Scania in $2002(\mathrm{~N}=556$ 912). We defined second-phase cases as cases for whom we had information on smoking status, diabetes, and medication for hypertension ( $\mathrm{N}=4$ 375). Information on these covariables for the controls was available from a public health survey $(\mathrm{N}=4716)$. As a proxy for exposure to air pollution, individual residential outdoor annual mean level of $\mathrm{NO}_{\mathrm{X}}$ was modelled. The median annual mean of $\mathrm{NO}_{\mathrm{X}}$ was $12.5 \mu \mathrm{g} / \mathrm{m}^{3}\left(5^{\text {th }}\right.$ and $95^{\text {th }}$ percentile: 5.6 and $30.0 \mu \mathrm{g} / \mathrm{m}^{3}$ ).

Results: In this study, using a complete study base, highquality ascertainment of cases, and individually modeled exposure, we did not observe any clear association between $\mathrm{NO}_{\mathrm{x}}$ and risk of ischemic stroke. As in all studies of this kind, misclassification of exposure was an issue. To further improve our study and decrease misclassification of exposure, questionnaires were sent out to around 5000 cases and 5000 controls, with $71 \%$ response rate among cases and $73 \%$ among controls.

Conclusions: Preliminary results and conclusions where questionnaire data are used, will be presented at the conference.

\begin{tabular}{ll}
\hline KARGER & (c) 2009 S. Karger AG, Basel \\
0251-5350/09/0332-0131\$26.00/0 \\
$\begin{array}{l}\text { Fax +4161306 1234 } \\
\begin{array}{l}\text { E-Mail karger@karger.ch } \\
\text { www.karger.com }\end{array}\end{array}$ & $\begin{array}{l}\text { Accessible online at: } \\
\text { www.karger.com/ned }\end{array}$
\end{tabular}




\section{$\mathrm{SS} 1 / 3$}

\section{Epidemiology of Stroke and a Community- Based Intervention Trial in China}

\author{
Wang W.Z. ${ }^{1}$, Hong Z. ${ }^{2}$, Yang O.D. ${ }^{3}$, Li S.C. ${ }^{4}$
}

${ }^{1}$ Beijing Neurosurgical Institute, Neuroepidemiology, Beijing, China, ${ }^{2}$ Shanghai Fudan University, Huashan Hospital, Shanghai, China, ${ }^{3}$ Zhongnan University, Xiangya Hospital, Changsha, China, ${ }^{4}$ Beijing Neurosurgical Institute, Beijing, China

Background: In order to investigate the morbidity and mortality of stroke in China and to explore the effective and suitable measures for reducing incidence and mortality of stroke in community populations.

Methods: Two large scale door-to-door surveys were conducted in 7 cities and 21 rural populations in China, and a community-based comprehensive intervention trial among 300,000 individuals in three urban communities in Beijing, Shanghai, and Changsha during 1991 to 2000.

Results: The average prevalence, incidence and mortality of stroke in urban populations were 719 per 100,000, 219 per 100,000 , and 116 per 100,000 respectively. The average prevalence, incidence and mortality in 21 rural areas were 394 per $100,000,185$ per 100,000 , and 142 per 100,000. Significant geographic differences were identified from the two surveys. Morbidity and mortality of stroke in populations in the north were substantially higher than that in southern parts of China. In the result of the community-based intervention trial, incidence and mortality of stroke in the intervention community decreased by $52.0 \%$ and 53.6\% respectively between 1991 and 2000. During the same period, incidence and mortality of stroke in the control community only decreased a little. The difference between the groups was statistically significant.

Conclusions: Stroke has been the leading cause of death in China in recent years. Use of comprehensive measures to prevent and manage patients with hypertension, together with regular education and health promotion activities in community populations, could reduce the incidence and mortality of stroke.

\section{SS1/4 \\ Randomisation is not Assuring Equalization if Male/Female Inclusion is Uneven. An Analysis Based on 25,607 Stroke Patients}

Olsen T.S. ${ }^{1}$, Andersen K.K. ${ }^{2}$

${ }^{1}$ Hvidovre University Hospital, The Stroke Unit, Hvidovre, Denmark, ${ }^{2}$ Technical University of Denmark, Informatics and Mathematical Modeling, Section for Statistics, Lyngby, Denmark

Background: Mortality is end-point in most randomized cardiovascular studies. We have demonstrated a lower post-stroke mortality in women compared to men especially in the early post stroke phase. In randomized cardiovascular studies the male/ female $(\mathrm{M} / \mathrm{F})$ ratio is often $2 / 3$ vs $1 / 3$. Hence, minor differences in the $\mathrm{M} / \mathrm{F}$ ratio between randomized groups may lead to differences in mortality between groups despite lack of treatment effect. We aimed to predict differences in mortality between two groups equalized in regard to age and stroke severity but with uneven $\mathrm{M} / \mathrm{F}$ inclusion differing $5 \%$ (62/38\% vs. 57/43\%; JAMA 1999 ; 21: 2019).

Method: Data are extracted from a Danish stroke registry of 25,607 patients with information on stroke severity and relevant stroke risk factors. Curves of one-month mortality in men/ women related to age and with fixed stroke severity scores (The Scandinavian Stroke Scale (SSS) 0-58) were established.

Results: One-month mortality adjusted for stroke severity was higher in men than women throughout life. One-month mortality at 70 years and 80 years of age (SSS fixed at 12) differed 9.8\% (M/F: 42.2\%/32.4\%) and 15.0\% (MF: 56.2\%/ 41.2\%) respectively. Assuming no treatment effect, an $\mathrm{M} / \mathrm{F}$ ratio of $62 / 38 \%$ in the treatment group and $57 / 43 \%$ in the placebo group the difference in mortality between groups turned out to be $1.3 \%$ at 70 years and $1.4 \%$ at 80 years of age.

Conclusion: If $\mathrm{M} / \mathrm{F}$ inclusion is uneven the randomized design does not assure equalization. We question inclusion of both sexes in randomized studies on severe stroke unless $\mathrm{M} / \mathrm{F}$ inclusion is even.

\section{SS1/5 \\ Improving Attention Deficits After Stroke: A Randomised Controlled Trial of Attention Process Training}

Barker-Collo S ${ }^{1}$, Feigin V ${ }^{2}$ Lawes $C^{3}$ Parag V ${ }^{4}$ on behalf of the START Study Team

${ }^{1}$ University of Auckland, Psychology, Auckland, New Zealand, ${ }^{2}$ Auckland University of Technology, National Centre for Stroke, Neuroscience and Neurorehabilitation, Auckland, New Zealand, ${ }^{3}$ The University of Auckland, Clinical trials Research Unit, Auckland, New Zealand, ${ }^{4}$ The University of Auckland, Clinical Trials Research Unit, Auckland, New Zealand

Background: Impairments in attention contribute to poor stroke outcomes, and attention is the basis for other areas of cognition (e.g., memory and communication). Attention Process Training (APT) has been shown using small samples to be effective in reducing attention deficits in persons with traumatic brain injury. There is no robust evidence for the effectiveness of APT in stroke patients.

Aims: To determine the effectiveness of APT in improving attention and health related quality of life (HRQoL) in stroke survivors at 6-months after the stroke.

Participants: Participants were 78 stroke patients admitted to rehabilitation units over an 18-month period, identified via neuropsychological assessment as having an attention deficit. Exclusion criteria: (1) inability to give informed consent; (2) severe cognitive deficits precluding participation; (3) medically unstable; (4) not fluent in English; or (5) any condition that could impact results. Participants were randomly allocated to receive 
30 hours of APT or standard care. This study had $80 \%$ power to detect significant 1SD change in theIVA-CPT.

Outcome: When adjusted for baseline IVA-CPT and stratification factors (age, gender, ethnicity, Barthel Index, group) change in IVA-CPT Full Scale Attention z-score for those receiving APT was on average 1.61 points greater than that of the standard care group $(\mathrm{p}=.004)$. These findings are of significance to evidencebased planning of rehabilitation and health service utilisation, and improving stroke outcomes; suggesting that APT is both a viable and effective means of improving attention in the months immediately after first-ever stroke.

\section{SS1/6 \\ Early Stroke and ABCD2 Stroke Risk Scores in a Large Transient Ischaemic Attack (TIA) Cohort}

Ghia D. ${ }^{1}$, Thomas P. ${ }^{2}$, Epstein D. ${ }^{3}$, Worthington J. ${ }^{4}$, Cordato D. ${ }^{4}$, Hodgkinson S. ${ }^{4}$, Beran R. ${ }^{4}$

${ }^{1}$ Liverpool Health Service, Department of Neurophysiology, Sydney, Australia, ²iverpool Health Service, Department of Neurology, Sydney, Australia, ${ }^{3}$ Bankstown Hospital, Sydney, Australia, ${ }^{4}$ The University of New South Wales, South West Sydney Clinical School, Sydney, Australia

Background: $\mathrm{The}_{\mathrm{ABCD}}^{2}$ stroke risk score was developed, using databases from the UK and USA, to identify TIA patients at high risk of early stroke. In some settings the score is used to determine urgency of TIA care. Our aim was to determine the rate of early ischaemic stroke after emergency diagnosis of TIA and predictive value of the $\mathrm{ABCD}^{2}$ score in an Australian cohort.

Method: Using administrative data-sets we identified first ever TIA emergency diagnoses admitted to two Sydney hospitals, where TIA admission was routine, during 3 years (2004 to 2006). $\mathrm{ABCD}^{2}$ scores were determined by expert record review. PostTIA presentations of stroke were sought in five surrounding hospitals and adjacent area health services. Positive predictive values of $\mathrm{ABCD}^{2}$ scores were determined.

Results: At Liverpoool and Bankstown hospitals there were 418 and 409 first ever TIA emergency diagnoses, respectively, from which 392 (93.8\%) and 397 (97.1\%) records could be expertly reviewed. Stroke rates in expert reviewed TIA at Liverpool and Bankstown hospitals were $0.5 \%$ and $2.3 \%$ at 30 days and $2.2 \%$ and $2.7 \%$ at 90 days respectively. In expertly reviewed TIA $(n=789)$ positive predictive value of moderatehigh $\mathrm{ABCD}^{2}$ scores $(>3)$ was just $0.9 \%$ at 30 days and $2.1 \%$ at 90 days. A low $\mathrm{ABCD}^{2}$ score had a positive predictive value of $2.6 \%$ and $3.4 \%$ at 30 and 90 days.

Conclusion: Stroke rates in this cohort of TIA patients admitted to hospital were low and the $\mathrm{ABCD}^{2}$ risk score had a low positive predictive value for early stroke occurrence.
SS2

Parkinson's Disease and Other Movement Disorders - Epidemiology and Management (Part 1)

August 28, 2009

11:30-12:30

SS2/1

Restless Legs Syndrome in a Population of
Northern Tanzania: A Cross-Sectional,
Community-Based Study

Winkler A. ${ }^{1}$, Trendafilova A. ${ }^{2}$, Meindl M. ${ }^{3}$, Tütüncü E. ${ }^{2}$, Kaaya J. ${ }^{4}$, Kassubek J. ${ }^{2}$, Schmutzhard E. ${ }^{3}$

${ }^{1}$ Departments of Interdisciplinary Palliative Care and Neurology, Ludwig-Maximilians-University, Munich, Germany, ${ }^{2}$ University of Ulm, Neurology, UIm, Germany, ${ }^{3}$ Medical University of Innsbruck, Neurology, Innsbruck, Austria, ${ }^{4}$ District General Hospital Babati, General Medicine, Babati, Tanzania, United Republic of Tanzania

Background: Restless legs syndrome (RLS) is a highly prevalent movement disorder. However, prevalences seem to vary amongst different ethnicities. To date, no community-based prevalence studies on RLS have been reported from the African continent.

Methods: We have conducted a cross-sectional, door-todoor study in northern Tanzania. Over a period of 16 months, 7,654 people aged 14 years and older were screened for RLS. Questionnaire and diagnoses were based on the Essential Criteria of the International Restless Legs Syndrome Study Group (IRLSSG). Sampling was performed according to the method of 'multistage cluster sampling'. People who screened positive where re-interviewed and physically examined by a specialist neurologist.

Results: During the screening phase, ten people answered 'yes' to at least one of the screening questions. After re-interviewing those people, the result was confirmed in five people only. After careful re-evaluation of the results and physical examination, one person was diagnosed with RLS, yielding a prevalence of $0.013 \%$ (age-adjusted $0.02 \%$ ).

Conclusion: The prevalence of RLS in a large northern Tanzanian population, as investigated by strict application of the IRLSSG Essential Diagnostic Criteria, was found to be very low (and thus much lower than that in Caucasians). If, beyond putative genetic factors, environmental factors were involved, has to be the subject of future studies on RLS prevalence and incidence in African populations. 


\section{SS2/2 \\ Physical Activities at Various Life Periods in Relation to Parkinson's Disease}

\author{
Xu Q. ${ }^{1}$, Park Y. ${ }^{2}$, Huang $X^{3}{ }^{3}$, Blair A. ${ }^{2}$, Hollenbeck A. ${ }^{4}$, \\ Schatzkin A. ${ }^{2}$, Chen $H$. \\ ${ }^{1}$ National Institute of Environmental Health Sciences, \\ Research Triangle Park, USA, ${ }^{2}$ National Cancer Institute, \\ Rockville, USA, 3Pennsylvania State University, Hersey, \\ USA, ${ }^{4}$ AARP, Washington DC, USA
}

Backgrounds: Experimental data showed that forced exercises before or after neurotoxin treatments saved dopaminergic neurons and alleviated movement abnormalities. However, epidemiological evidence on exercise and Parkinson's disease (PD) risk is preliminary and inconsistent.

Methods: We evaluated physical activities over four lifeperiods as reported in 1996-1997 in relation to PD among 214905 participants of the NIH-AARP Diet and Health Study. PD cases were identified from the 2004-2006 follow-up survey and were classified by the year of first diagnosis.

Results: The primary analyses included 1,161 PD cases diagnosed after 1995. Higher moderate to vigorous activities in two recent periods were each associated with lower PD occurrence. Compared with participants who never or rarely excised in the past 10 years before the 1996-1997 survey, the odds ratio (OR) for those who spent $>7$ hours / week on moderate to vigorous activities was 0.66 (95\% confidence interval: $0.54-0.81, \mathrm{P}$ for trend $<0.0001)$. The corresponding OR for age period 35-39 was $0.68(0.55-0.85$, $\mathrm{P}$ for trend $=0.01)$. The results were similar for men and women and in analysis excluding cases diagnosed before 2000. Further, participants whose moderate to vigorous activities remained high or increased from medium to high during these two periods had statistically lower PD risk than those who remained inactive. The corresponding ORs were $0.56(0.43-0.74)$ and 0.60 (0.44-0.82) respectively. Neither moderate to vigorous activities at early ages nor light activities in any life periods were significantly associated with PD occurrence.

Conclusion: The results suggest that vigorous exercise protects against $\mathrm{PD}$.

\section{$\mathrm{SS} 2 / 3$ \\ Idiopathic Parkinson's Disease and Hypercholesterolemia: Results from a Case-Control Study}

D'Amelio M., Ragonese P., Di Benedetto N., Callari G., Famoso G., Terruso V., Aridon P., Savettieri G.

Dipartimento di Neuroscienze Cliniche, Università degli

Studi di Palermo, Palermo, Italy

Objective: To assess the co-occurrence of hyperlipidaemia in patients with Parkinson s disease (PD).

Background: recent findings suggest a role of lipid and cholesterol metabolism in the pathogenesis of PD.
Methods: We designed a case-control study to investigate the co-occurrence of hyperlipidaemia in PD patients. PD diagnosis was based on the presence of two out of four cardinal signs in people who were not on anti-parkinsonian therapy, while at least one sign was required in individuals who were taking antiparkinsonian therapy. Vascular and other parkinsonism were excluded. PD individuals were matched to PD free individuals (1:1). Hyperlipidaemia diagnosis was ascertained by a structured questionnaire and use of a cholesterol- lowering drugs was also investigated. Odds Ratios (OR) were calculated and adjusted for known risk factors.

Results: Three-hundred-ten pairs (51.6\% women) were included in the study. PD patients had a mean age at interview of 66.7 years, mean age at PD onset 60.7 years and mean PD duration 5.9 years. Twenty-six PD patients $(8.4 \%)$ and 35 controls $(11.3 \%)$ were affected by hyperlipidaemia. Univariate analysis showed a slight inverse association between PD and hypercholesterolemia (OR 0.7; 95\% CI 0.4, 1.2). The inverse association was stronger in men (OR 0.6 ; 95\% CI $0.2,1.3)$, than in women (OR $0.9 ; 95 \%$ CI 0.4, 1.8).

Conclusions: Our findings support previous evidences of an inverse association between PD and hyperlipidaemia. Whether PD is etiologically linked to lower level of serum lipid or cholesterol-lowering agents have a neuroprotective effect deserve further studies.

\section{SS2/4 \\ Validation of a Screening Tool for Tic Disorders}

Cubo E. 1, Trejo Gabriel y Galán J.M. 1, Cordero Guevara J. ${ }^{2}$, Saez Velasco S. ${ }^{3}$, Ausin Villaverde V. ${ }^{3}$, Delgado Benito V. ${ }^{3}$, Macarrón Vicente J. ${ }^{1}$, Martín Santidrián A. ${ }^{1}$, Benito J. , Louis E.D. ${ }^{5}$

${ }^{1}$ Hospital General Yague, Neurology, Burgos, Spain, ${ }^{2}$ Primary Health Management, Burgos, Spain, ${ }^{3}$ Hospital General Yague, Burgos, Spain, ${ }^{4}$ Hospital 12 de Octubre, Madrid, Spain, ${ }^{5}$ College of Physicians and Surgeons, Columbia University, New York, USA

Introduction: Tic disorders are fluctuating, neurodevelopmental disorders. Prevalence estimates are divergent, likely related to differences in methodology.

Objective: To validate a screening method for tic disorders in mainstream (MS) and special education centers (SEC).

Methods: This was a pilot, community-based survey designed to quantify the prevalence of tics in youngsters (8-13 years) in Burgos, Spain. Possible tic disorder was defined based on an ad hoc screening questionnaire administered to teachers, parents, and observation (trained observers). Ascertained diagnosis (gold standard) was assigned by the neurologist (DSM-IV TR, excluding functional impairment criteria). Sensitivity, specificity, and positive/negative predictive value (PPV, NPV) were calculated.

Results: Of 205 potential subjects, $67 \%$ in $\mathrm{MS}$ and $51 \%$ in SEC consented to participate. Tic disorders were found in 12/64 $(19 \%)$ children in MS and 11/54 (20\%) children in SEC. The sensitivity of tic disorder was 58\% (MS) and 36\% (SEC) for observ- 
ers, $40 \%$ (MS) and 73\% (SEC) for teachers, and 85\% (MS) and $44 \%$ (SEC) for parents. By combining data from all the 3 screening sources, the sensitivity, PPV and NPV were $92 \%, 38 \%$ and $97 \%$ in MS, and $82 \%, 20 \%$, and $81 \%$ in SEC.

Conclusions: Tic disorders were frequent among these schoolchildren. For epidemiological studies of fluctuating disorders such as tics, combined multiple sources appear to be adequate for screening. This methodology may be more valid in MS compared to SEC settings. However, parents and teachers seem to be the best single screening source in MS and SEC, respectively.

\section{SS2/5}

Analysis of Surgical Intrathecal [i.t.] Baclofen [ITB] Implant Results Emphasizing Revision Surgery in a Mixed Pediatric/Adult Population

\section{Awaad Y. \\ King Fahad Medical City, Pediatric Neurology, Riyadh, Saudi Arabia}

Introduction: Increasingly, spasticity is managed with ITB using surgically implanted programmable pumps [Medtronic, Inc.]. ITB revision surgery unrelated to programmable pump end-of-life is not uncommon, requiring special attention during pre-, intra-, and post-operative management. We retrospectively reviewed our recent revision surgery experience based upon 1) primary implants at our own institution ['primary-implant-patients'], and 2) operative revisions for cases originally implanted outside our institution ['revision-only-patients'].

Methods and Materials: Since 2002 we treated 41 patients [21F/20M; 22 children $(14 \mathrm{~F} / 8 \mathrm{M})$ vs. 19 adults $(7 \mathrm{~F} / 12 \mathrm{M}) ; 30$ 'primary-implant-patients': 16 children $(10 \mathrm{~F} / 6 \mathrm{M})$ vs. 14 adults $(5 \mathrm{~F} / 9 \mathrm{M}) ; 11$ 'revision-only-patients': 6 children $(4 \mathrm{~F} / 2 \mathrm{M})$ vs. 5 adults $(2 \mathrm{~F} / 3 \mathrm{M})]$. Clinical charts, operative and imaging reports were reviewed to evaluate reasons for revision surgery and diagnostic work-up requirements.

Results: Eight of 30 'primary-implant-patients' required 14 revisions and 7 of 11 'revision-only-patients' needed 13 procedures. Seven patients with slowly increasing baclofen-resistant spasticity had either 1) unsuspected pump-catheter connector defects $[\mathrm{N}=4]$ with a subcutaneously dislocated i.t. catheter $[\mathrm{N}=1], 2)$ an $\mathrm{X}$-ray-documented pump-catheter connector defect $[\mathrm{N}=1]$ or 3) an X-ray-demonstrated fractured catheter with i.t. fragment $[\mathrm{N}=2]$ requiring laminectomy $[\mathrm{N}=1]$. Injection studies revealed i.t. peri-catheter arachnoiditis $[\mathrm{N}=1$; managed without laminectomy], and connector-related dye leakage [ $\mathrm{N}=3$ ]. Implant infections occurred in 4 cases [3 were multiply pre-operated]. Scintigraphy revealed occult CSF leakage $[\mathrm{N}=1]$. Intrinsic pump failure was rare $[\mathrm{N}=1]$.

Conclusions: ITB, although very gratifying, has a high, predominantly technique-related complication incidence during implant life. Meticulous surgical technique, high clinical suspicion, appropriate work-up, and timely surgical management are emphasized to reduce surgical ITB complications.

\section{SS2/6 \\ Metric Properties of the SCOPA-AUT Brazilian Version}

\author{
Carod Artal F.J. ${ }^{1}$, da Silveira Ribeiro L. ${ }^{1}$, Kummer W. ${ }^{1}$, \\ Martínez Martin P. \\ ${ }^{1}$ Sarah Hospital, Neurology, Brasilia, Brazil, ${ }^{2}$ National \\ Center for Epidemiology and CIBERNED, Carlos III \\ Institute of Health, Neuroepidemiology, Madrid, Spain
}

Background/Objective: Cross-cultural adaptation and assessment of the psychometric properties of the Scales for Outcomes in Parkinson's disease-Autonomic questionnaire (SCOPA-AUT) Brazilian version.

Methods: Consecutive Parkinson's disease (PD) patients able to reply questionnaires were evaluated by means of the Scales for Outcomes in Parkinson's disease-Motor scale (SCOPA-M), Cognition scale (SCOPA-COG), Hoehn and Yahr staging (H\&Y), Non-motor Symptoms Scale (NMSS), Berg Balance Scale, PD Sleep Scale (PDSS), PD Questionnaire (PDQ-39) and SCOPAAUT. The following metric attributes were explored for the SCOPA-AUT: acceptability, scaling assumptions, reliability, precision, and construct validity.

Results: 150 patients were assessed (mean age $63.1 \pm 11.1$ years; $56.7 \%$ males; mean duration of illness $8.7 \pm 5.3$ years; $H \& Y$ median: 2). Mean SCOPA-AUT score was 23.0 \pm 11.2 . Floor and ceiling effect were not detected. SCOPA-AUT domains reached Cronbach alpha from 0.56 to 0.81 . Intraclass correlation coefficient for SCOPA-AUT total score was 0.71 , whereas weighted kappa for individual items ranged from 0.15 to 0.71 (14 items, $61 \%$, showing values $>0.60)$. Standard error of measurement value for SCOPAAUT was 6.04 (0.53 to 2.3 for the domains). The SCOPA-AUT total score significantly correlated with the NMSS total score $\left(r_{S}=0.65\right)$ and the correlation between corresponding dimensions of both scales resulted moderate to high $\left(\mathrm{r}_{\mathrm{S}}=0.46-0.67\right.$; all, $\mathrm{p}<$ 0.0001). SCOPA-AUT correlated at a moderate level with the following scales: $\mathrm{H} \& \mathrm{Y}\left(\mathrm{r}_{\mathrm{S}}=0.35\right)$, SCOPA-MS total score $\left(\mathrm{r}_{\mathrm{S}}=0.39\right)$, $\operatorname{PDSS}\left(\mathrm{r}_{\mathrm{S}}=-0.60\right)$ and PDQ-39 Summary Index $\left(\mathrm{r}_{\mathrm{S}}=0.61\right)(\mathrm{all}, \mathrm{p}<$ $0.0001)$. SCOPA-AUT significantly increased as H\&Y increased (Kruskal-Wallis, $\mathrm{p}<0.0001$ ).

Conclusions: The SCOPA-AUT Brazilian version is a reliable and valid questionnaire to evaluate autonomic dysfunction in PD. 
SS3

Multiple Sclerosis Epidemiology

August 28, 2009

16.00-17.00

SS3/1

Epidemiology of Multiple Sclerosis (MS) in Ireland: Prevalence in Dublin, Wexford and Donegal in Relation to Vitamin D Status

\author{
Lonergan R. ${ }^{1}$, Kinsella K. ${ }^{1}$, Fitzpatrick P. ${ }^{2}$, Duggan M. ${ }^{1}$, \\ Jordan S. ${ }^{1}$, Brady J. ${ }^{3}$, Murray B. ${ }^{3}$, Mc Kenna M. ${ }^{3}$, \\ Hutchinson M. ${ }^{1}$, Tubridy N. \\ 'St. Vincent's University hospital, Neurology, Dublin, \\ Ireland, ${ }^{2}$ School of Public Health and Population Science, \\ University College Dublin, College of Life Sciences, \\ Dublin, Ireland, '3St. Vincent's University hospital, \\ Metabolism, Dublin, Ireland
}

Background: Reports suggest increasing MS prevalence. Prevalence increases with latitude, and this latitudinal variation was demonstrated in Ireland in 2001.Genetic factors contribute but vitamin $\mathrm{D}$ deficiency is a possible co-factor.

We hypothesised that, in Ireland:

1 - Vitamin D is lower in patients with MS than in controls.

2- Greater prevalence at higher latitudes (Donegal) relates to lower vitamin D levels in comparison to southern areas (Wexford, South Dublin).

3- Prevalence is greater than in 2001.

Methods: Patients and controls were identified in Donegal, Wexford and South Dublin through multiple sources. They attended research clinics from November 2007 to March 2008. Serum samples were taken for vitamin D measurement.

Results: Prevalence significantly increased in Donegal (252.5/100,000: 95\%CI 224.3-283.7) compared with 2001 (184.6/ 100,000: CI 162- 209.5). In Wexford there was a non-significant increase in prevalence. Prevalence is significantly higher in Donegal than in Wexford (125.6/100,000: 95\%CI 106.7-147.8 p $<0.0001)$ and South Dublin (99.3/100,000: 95\%CI 81.3-121.2, p $<0.0001)$.

Overall, mean vitamin D levels were low in both patients $(38.57 \mathrm{nmol} / \mathrm{L})$ and controls $(36.41 \mathrm{nmol} / \mathrm{L}$; winter normal $>50 \mathrm{nmol} / \mathrm{L}$ ) and did not differ significantly However, levels were significantly higher in South Dublin (mean 50.74nmol/L, $\mathrm{p}<$ 0.0001 ), the area with lowest prevalence

Conclusion: Vitamin D deficiency is common in Ireland. Latitudinal variation in MS probably relates more to genetic factors (HLA) than to vitamin D levels alone. Dietary vitamin D supplementation may explain higher levels in South Dublin.

\section{SS3/2}

The Role of Relapses Affecting the LongTerm Disability Outcome in the London Ontario Multiple Sclerosis Database

Scalfari A. ${ }^{1}$, Degenhardt A. ${ }^{2}$, Neuhaus A. ${ }^{3}$, Harner $N^{3}{ }^{3}$ Seemueller S. ${ }^{3}$, Nicholas R. ${ }^{1}$, Muraro P. ${ }^{1}$, Daumer M. ${ }^{3}$, Ebers G. $^{2}$

${ }^{1}$ Imperial College, Cellular and Molecular Neuroscience, London, United Kingdom, ${ }^{2}$ Oxford University, Oxford,

United Kingdom, ${ }^{3}$ Sylvia Lawry Centre, Munich, Germany

Background: The prevention of relapses is now a therapeutic reality. We aim to reassess the effect of relapses on the development of advanced disability.

Methods: We used the updated version of the London Ontario database comprising 28,000 patient-years of follow-up. Life table analysis was used to compare survival in patients stratified according to number of relapses in the first two years $(1 ; 2$; $>2)$, first inter-attack interval $(0-2 ; 3-5 ; \geq 6$ years $)$ and number of relapses during the relapsing remitting phase of the disease (1-2; $3-4 ; \geq 5)$.

Results: The survival analysis confirmed that the mean time to the assignment of each disability score from the onset of the disease was significantly longer in patients with a lower number of relapses. However, once DSS 6 was reached no differences between groups were observed in further accumulating disability. Patients with a longer time to the second attack took also longer to reach high disability levels and to convert to secondary progressive MS. No differences in attaining the endpoints analyzed were observed when patients were stratified according to the total number of attacks during the relapsing remitting phase.

Conclusions: Relapses during the first two years and the length of the first inter-attack interval are important determinants of time to high DSS levels; their predictive role disappears once established (DSS 6) disability occurs. The total number of relapses before the onset of progression didn't affect the long term disability outcome. These results confirm and extend what was reported after 12 years of observation 


\section{SS3/3}

Multiple Sclerosis in Northeast of Catalonia: An Epidemiologic Study

\author{
Carmona O. ${ }^{1}$ Masuet C. ${ }^{2}$, Montero C. ${ }^{1}$, Díaz L. ${ }^{1}$, \\ Forment J.C. ${ }^{3}$, Caula J. ${ }^{4}$, Ramió-Torrentà L. ${ }^{5}$, Perkal H. ${ }^{6}$, \\ Paz M. ${ }^{7}$, Primary Health Care Practitioners of Alt Empordà \\ ${ }^{1}$ Fundació Salut Empordà, Neurology, Figueres, Spain, \\ ${ }^{2}$ Hospital de Bellvitge, Public Health Department, \\ L'Hospitalet, Spain, ${ }^{3}$ Fundació Salut Empordà, Primary \\ Health Care Coordinator, Figueres, Spain, ${ }^{4}$ CS, Public \\ Health Technician, Girona, Spain, ${ }^{5}$ Hospital Josep Trueta, \\ Neurology, Girona, Spain, ${ }^{6}$ Hospital Vall d'Hebró, MS Unit,

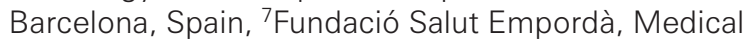 \\ Department Chief, Figueres, Spain
}

Background: Alt Empordà is a well-defined sanitary area of 124.000 habitants, belonging to Girona district, which has experienced an overgrowth of population due to immigration in the last ten years.

\section{Objectives:}

- To obtain the prevalence of Multiple Sclerosis (MS) in $31^{\text {st }}$ December 2006 and a retrospective diagnose incidence data between 2001 and 2006

- To analyse differences respect MS evolution between local and immigrant patients.

Methods: We obtained MS diagnoses codes of all public primary health care sites and the reference area Hospital in Figueres. These data were confirmed by MS referees in Girona and Barcelona districts. Inclusion criteria were: 1) Definite MS diagnosis 2) Patients born and living in Alt Empordà during study period or immigrants living in our country before 15 years'old. Demographic and clinical data were recorded.

Results: Seventy-eight patients with MS were identified: $63 \%$ were women and $74 \%$ were born in our district. Median age was 48 years and mean duration of MS was 10 years. Median EDSS was 2.0 .

The prevalence rate was 62.3 patients/100.000 habitants and the mean period diagnose incidence was $5 / 100.000$ habitants per year. Median EDSS between Alt Empordà and immigrant patients were different ( 2 versus $4.5, \mathrm{p}=0.3$ ) and was maintained when we analysed separately UE and North-African population.

Conclusions: Prevalence and incidence data obtained in our study are congruent with epidemiologic studies published in Spain. A more aggressive form of multiple sclerosis in immigrant population respect to local patients is suggested.

\section{SS3/4 \\ GERONIMUS Study: Progression Rates to Multiple Sclerosis According to McDonald and Poser Criteria in Patients with Clinically Isolated Syndrome}

\author{
D'Alessandro R. ${ }^{1}$, Baldin E. ', Benedetti M.D. ${ }^{2}$, Delaj L. ', \\ Galeotti M. ${ }^{3}$, Granella F.4, Leone M. ${ }^{5}$, Lugaresi A. ${ }^{6}$, \\ Motti L. ${ }^{7}$, Neri W. ${ }^{8}$, Santangelo M. ${ }^{9}$, Tola M.R. ${ }^{10}$, \\ Vignatelli L. ${ }^{1}$, Guidolin L. ${ }^{11}$, on behalf of GERONIMUS \\ Group \\ ${ }^{1}$ Institute of Neurological Sciences, Bologna, Italy, \\ ${ }^{2}$ Neurological Institute, Verona, Italy, ${ }^{3}$ Neurological Unit, \\ Lugo, Italy, ${ }^{4}$ Institute of Neurological Sciences, Parma, \\ Italy, ${ }^{5}$ Institute of Neurological Sciences, Novara, Italy, \\ ${ }^{6}$ Neurological Unit, Chieti, Italy, ${ }^{7}$ Neurological Unit, Reggio \\ Emilia, Italy, ${ }^{8}$ Neurological Unit, Forlì, Italy, ${ }^{9}$ Neurological \\ Unit, Carpi, Italy, ${ }^{10}$ Institute of Neurological Sciences, \\ Ferrara, Italy, ${ }^{11}$ Biogen Dompé, Milano, Italy
}

Objectives: GERONIMUS is a multicentre prospective cohort study aiming to evaluate the prognosis of patients with symptoms suggestive of Multiple Sclerosis (MS). We present the progression rate to MS according to McDonald Criteria and Poser Criteria after two years of follow-up.

Methods: Patients with symptoms suggestive of MS in the previous six months were included over a two-year period (20052007), after a standardised MRI examination and exclusion of other pathological conditions. Neurological evaluation was performed at baseline and yearly in the follow-up phase. KaplanMeier estimates were used to construct progression to MS curve according to Poser criteria (clinically definite MS - CDMS) and 2001 McDonald criteria.

Results: 196 patients were enrolled (64 M, 132 F, median age 31 years, range $17-55$ years); $88 \%$ of subjects completed 2 years of follow-up. The diagnosis of MS was established by McDonald Criteria in $54 \%$ of patients and by Poser Criteria in $34 \%$. The median number of MRI lesions at enrolment was 8.0 (25-75\%ile $3-17)$ in the whole sample, 11.5 (25-75\%ile 5-18) in the patients with CDMS, 12 (25-75\%ile 6-19) in patients with McDonald MS.

Conclusion: The findings of this study will be discussed in view of both prospective observational studies on conversion rate to CDMS of patients with Clinically Isolated Syndrome (CIS) and the prognosis of patients in the placebo arms of randomized controlled trials (RCTs) testing immunomodulatory treatment. A preliminary analysis seems to disclose a better prognosis in the GERONIMUS study patients compared to RCTs. 


\section{SS3/5}

\section{Adjunct Glucosamine Sulfate Do Not Reduce the Risk of Relapsing-Remitting Multiple Sclerosis Progression}

\author{
Janghorbani M. ${ }^{1}$, Shaygannejad V. ${ }^{2}$, Savoj M.R. ${ }^{2}$, Ashtari $F^{2}$ \\ ${ }^{1}$ Isfahan University of Medical Sciences, Epidemiology \\ and Biostatistics, Isfahan, Iran, Islamic Republic of, \\ ${ }^{2}$ Department of Neurology, Medical School, Isfahan \\ University of Medical Sciences, Isfahan, Iran, Islamic \\ Republic of Iran
}

Objective: The aim of this preliminary study was to evaluate the effect of glucosamine sulfate in combination with current disease modifying therapy in the prevention of progression of relapsing-remitting multiple sclerosis (RRMS).

Methods: A phase II double-blind placebo-controlled randomized clinical trial conducted between February and October 2007. 97 definite RRMS patients age 17 to 55 years were randomly allocated to receive a 6 -months treatment course of either oral glucosamine sulfate $(1000 \mathrm{mg} /$ day $)$ or placebo in combination with disease modifying therapy. Response to treatment was assessed at 6 months after start of therapy. Primary and secondary outcome measures included changes in mean EDSS and number of relapse.

Results: Of the 46 patients treated with glucosamine sulfate, the mean (SD) of relapse rate decreased from $1.1(0.7)$ at baseline to $0.4(0.5)$ at the end of study period $(\mathrm{P}<0.05)$. Correspondingly, in the 51 patients treated with placebo, the mean (SD) of relapse rate did not change. After 6 months, $63.0 \%$ of patients receiving glucosamine sulfate remained relapse free compared with $54.9 \%$ of those given placebo $(\mathrm{P}>0.05)$. The average EDSS at the end of trial did not changed between glucosamine sulfate and placebo group (mean difference, $-0.1 ; 95 \% \mathrm{CI},-0.5,0.2$ ).

Conclusion: Adding glucosamine sulfate to routine disease modifying therapy had no significant effect on the relapse rate or disease progression in the treatment period. A larger phase III multicenter study of glucosamine sulfate in RRMS is warranted in order to more definitely assess the efficacy of this intervention.

\section{SS3/6}

\section{Chronic Neuro-Ophthalmological Manifestations in Multiple Sclerosis: Epidemiological Study of $\mathbf{3 0 0}$ Patients}

\author{
Jasse L. ${ }^{1}$, Vukusic S. ${ }^{2}$, Piras C. ${ }^{3}$, Vardanian $C^{3}{ }^{3}$, Bernard M. ${ }^{3}$, \\ Pélisson D. ${ }^{1}$, Vighetto A. ${ }^{4}$, Tilikete $C^{4}$ \\ ${ }^{1}$ INSERM U864, Bron, France, ${ }^{2}$ Hôpital Neurologique et \\ Neurochirurgical P. Wertheimer, Service de Neurologie A, \\ Bron, France, ${ }^{3}$ Hôpital Neurologique et Neurochirurgical P. \\ Wertheimer, Service de Neuro-Ophtalmologie, Bron, \\ France, ${ }^{4}$ INSERM U864, Hôpital Neurologique et \\ Neurochirurgical P. Wertheimer, Service de Neuro- \\ Ophtalmologie, Bron, France
}

Among chronic symptoms in multiple sclerosis (MS), visual deficits are observed in $40 \%$ of patients. However, physiopatho- logical origins and functional repercussion of such troubles are not well known.

The aim of this study is to focus on chronic visual deficit (CVD) in a large group of MS patients, in determining its frequency, functional repercussion and physiopathological origin.

Among three hundred questioned MS patients, the patients who complained of CVD passed an exhaustive neuro-ophthalmological examination including visual function and oculomotor tests. Descriptive analyses were conducted for all data.

$34 \%$ of MS patients complained of CVD and the most frequently of blurred vision. Visual acuity in our 81 patients was 20/32. Monocular visual field was impaired in $21 \%$ of patients and binocular in $44 \%$. $17 \%$ disclosed impaired colour perception for one eye and $47 \%$ for two eyes. Contrast sensitivity was impaired in one eye for $28.5 \%$ and for two eyes in $21 \%$. Oculomotor and orthoptic examination showed an oculomotor paresis in $57 \%$ of patients and abnormal spontaneous eye movements in $79 \%$ of patients.

Principal mechanisms of CVD were an optic neuropathy $(60 \%)$ which was binocular in $38 \%$, an internuclear ophthalmoplegia $(46 \%)$ or gaze-evoked nystagmus $(46 \%)$.

11 of 81 patients disclosed signs of bilateral optic neuropathy without previous attack of optic neuritis. 11 out of 81 patients disclosed isolated oculomotor troubles without any impairment of afferent visual pathway.

Our study shows numerous patients complained of CVD. Moreover, some optic neuropathy could be of chronic evolution, and several patients disclosed exclusively oculomotor deficit.

\section{SS4}

Epidemiology of Traumatic Brain Injury

August 28, 2009

17:00-18:00

SS4/ 1

\section{Long-Term Survival After Traumatic Brain Injury (TBI): A Population-Based Analysis Using Severity-Matched, Non-TBI Injured Controls}

Brown A. ${ }^{1}$, Leibson C. ${ }^{2}$, Mandrekar J. ${ }^{3}$, Malec J. , Ransom J. ${ }^{3}$

${ }^{1}$ Mayo Clinic, Physical Medicine and Rehabilitation, Rochester, USA, ${ }^{2}$ Mayo Clinic, Epidemiology, Rochester, USA, ${ }^{3}$ Mayo Clinic, Biomedical Statistics \& Informatics, Rochester, USA, ${ }^{2}$ Rehabilitation Hospital of Indiana, Indianapolis, USA

Background: The contribution of non-head injuries to reduced long-term survival following TBI is unknown. 
Methods: From all 45,791 Olmsted County, MN residents with diagnoses indicating potential TBI 1985-2000, 7,175 were randomly sampled for record review to characterize TBI events as severe, mild or no TBI. For each confirmed case $(\mathrm{N}=1,429)$, one age-sex-matched non-TBI control was selected from the general population (regular controls). For each case seen in Emergency Department (ED) with accompanying non-head injuries $(\mathrm{N}=221)$, a severity score for non-head injuries was calculated and two 'special' controls (seen in ED with similar severity score) were assigned. Subjects' vital status was followed through 12/31/2008. Survival was analyzed 0-6 months and among 6-month survivors, overall and stratified by TBI severity and age $(<16,16-64$, and $65+$ ). Cases were compared with controls, first considering 1429 regular controls and second considering 1208 regular plus 442 special controls.

Results: Within 0-6 months, overall mortality was substantially increased for TBI cases vs. controls, regardless of control assignment. Among 6-month survivors, analyses consisting only of regular controls revealed significantly increased mortality for cases, overall (hazard ratio, 95\% confidence interval $=1.57,1.08$ 2.27) and among mild TBI cases age 16-64 (1.93, 1.01-3.68). In analyses including special controls, significant differences among 6-month survivors disappeared, overall $(1.12,0.81-1.55)$ and among mild cases age 16-64 (1.32, 0.76-2.28). No significant increases in mortality among 6-month survivors were observed for TBI cases age $<16$ or $65+$.

Conclusion: When investigating long-term survival after TBI, it is important to adjust for injuries that accompany TBI.

\section{SS4/2}

\section{Functional Level During Sub-Acute Rehabilitation After Traumatic Brain Injury: Course and Predictors}

\author{
Sandhaug M. ${ }^{1}$, Andelic N. ${ }^{2}$, Vatne A. ${ }^{3}$, Seiler S. ${ }^{4}$, \\ Mygland $A{ }^{5}$ \\ ${ }^{1}$ University of Agder, Faculty for Health- and Sports, \\ Kristiansand, Norway, ${ }^{2}$ Department of Physical Medicine \\ and Rehabilitation, Oslo University Hospital Ulleval, Oslo, \\ Norway, ${ }^{3}$ Department of Neurology, Sørlandet Hospital, \\ Kristiansand, Norway, ${ }^{4}$ University of Agder, Faculty of \\ Health- and Sports, Kristiansand, Norway, ${ }^{5}$ Department of \\ Habilitation, Hospital for Rehabilitation, Oslo University \\ Hospital, Kristiansand, Norway
}

Background: To describe the functional level during subacute rehabilitation after severe and moderate traumatic brain injury (TBI), and to evaluate the impact of pre-injury and injuryrelated factors as predictors of early recovery.

Methods: A prospective study of 55 patients with moderate $(n=21)$ and severe $(n=34)$ TBI who received specialized, sub-acute inpatient rehabilitation. Functional level was assessed by the Functional Independence Measure (FIM). Possible predictors were analyzed in a regression model using FIM total score at discharge as outcome.

Results: At discharge from sub-acute rehabilitation, on average $53( \pm 24)$ days post-injury, $95 \%$ of patients with moderate
TBI, and $62 \%$ with severe TBI had a good functional level with a FIM total score of 109-126. Among 34 patients with severe TBI $24 \%$ had a poorer functional level with a FIM total score $<72$ and 1 patient did not improve. Predictors of functional level were FIM total score at rehabilitation admission $(\mathrm{B}=0.411)$, Glasgow Coma Scale (GCS) score at rehabilitation admission $(B=6.127)$, length of post-traumatic amnesia (PTA) $(\mathrm{B}=-0.140)$, and length of stay (LOS) in the rehabilitation unit $(\mathrm{B}=0.318)$.

Functional measures at admission and discharge from sub-acute rehabilitation

\begin{tabular}{lrrr}
\hline & Amission rehab & Discharge rehab & p-value \\
\hline Moderate TBI $(\mathrm{n}=21)$ & & & \\
FIM total & 108 & 122 & 0.000 \\
FIM-M & 81 & 90 & 0.002 \\
FIM-COG & 27 & 32 & 0.000 \\
Severe TBI $(\mathrm{n}=34)$ & & & \\
FIM total & 76 & 97 & 0.000 \\
FIM-M & 57 & 73 & 0.000 \\
FIM-COG & 20 & 25 & 0.000 \\
\hline
\end{tabular}

Conclusion: A high FIM score at admission, a high GCS score at admission, a short PTA period, and long stay in the rehabilitation unit are positive predictors of functional level at discharge from sub-acute rehabilitation.

\section{SS4/3 \\ Profiles of Brain Trauma Patients in A/E Department of a Hospital in Jakarta, Indonesia January-June 2005}

Wreksoatmodjo $B$.

Atmajaya Catholic University, Neurology, Jakarta Utara, Indonesia

Background: Head/brain trauma are among the most frequent cases seen in A/E department of a hospital; and the incidence is expected to increase, particularly in cities. To be optimally managed, we need to know the characteristics of trauma cases.

Methods: Patients' data obtained from admission record in one of the hospitals in Jakarta, Indonesia during 6-month period (January-June 2005); characteristics of patients, GCS (Glasgow Coma Score), diagnosis, CT scan results as well as the final management were recorded. Data was then tabulated.

Results: There were 172 trauma cases among 7835 admissions; the incidence of brain/head trauma was $2.20 \%$, mostly affected men (102-59.30\%) in productive age (21-50 year-old $59.89 \%)$. The majority of cases are Mild Head Injury (70.93\%). Brain CT scan was done in most cases $(79.65 \%)$ and reveal that contusional bleeding / hemorrhage can be found even in clinically mild cases (11 in 13 cases); brain hemorrhage found was consisted of epidural hemorrhage (11 cases),subdural hemorrhage (17 cases), intracerebral hemorrhage ( 7 cases) and subarachnoid hemorrhage (9 cases); multiple hemorrhages also found. Skull fractures was found in 21 cases.

Conclusion: Brain/head trauma mostly affected men in productive age, and in milder form.Brain CT scan should be done 
in most cases because it can reveal contusional bleeding even in mild cases.

SS4/4

\section{Epidemiology and Classification of Head Injury Sequelae}

\author{
Likhterman L. ${ }^{1}$, Nepomnyashy V. ${ }^{2}$, Akshulakov S. ${ }^{3}$ \\ ${ }^{1}$ Department of Neurotrauma, The Burdenko \\ Neurosurgery Institute, Moscow, Russian Federation, \\ ${ }^{2}$ The Burdenko Neurosurgery Institute, Moscow, Russian \\ Federation, ${ }^{3}$ Institute of Neurosurgery, Astana, Kazakhstan
}

Introduction: Head injury sequelae are accumulated in aging population. This is a public health problem. But unlike acute head injury their epidemiology is almost unknown and systematic classification is non-existent.

Aims: to develop clinico-pathological classification of head injury sequelae and to study the incidence of its surgically relevant forms.

Material and Methods: Our study is based upon epidemiological and clinical analysis of 4000 cases of head injury sequelae confirmed by CT and/or MRI.

Results: We proposed a clinico-pathological classification which includes three types of head injury sequelae: 1) tissue (local and diffuse brain atrophy, meningo-cerebral scars, skull defects etc.), CSF (hydrocephalus, porencephalus, CSF fistulae etc) and vascular (posttraumatic aneurisms, carotid-cavernous fistulae, chronic subdural haematomas etc.). According to our populationbased epidemiological study of acute head injury and its follow-up the incidence of surgically treatable forms of head injury sequelae is $10,6 \pm 0,7$ for 100.000 head injury survivors. A considerable number of patients had two or more clinical forms of head injury sequelae (an average number of surgically treatable forms per patient was 1, 44 for new cases).

Conclusion: Extrapolation of our results suggests at least 25.000 cases of surgically treatable head injury sequelae in Russia and more than 3.000 cases in Kazakhstan. In depth study of epidemiology of head injury sequelae is needed.

\section{SS5}

Migraine and Other Pain Disorders Epidemiology

August 29, 2009

11:30-12:30

\section{SS5/1}

\section{Prevalence of Migraine, Probable Migraine and Tension Type Headache in Croatian Population}

\author{
Vuković V. ${ }^{1}$, Pavelin S. ${ }^{2}$, Janculjak D. ${ }^{3}$, Ivanković M. ${ }^{4}$, \\ Plavec D. ${ }^{5}$, Lovrenčić Huzjan A. ${ }^{1}$, Demarin V. ${ }^{1}$ \\ 'Department of Neurology, University Hospital 'Sestre \\ milosrdnice', Zagreb, Croatia, '2Department of Neurology, \\ University Hospital Split, Split, Croatia, ${ }^{3}$ Department of \\ Neurology, University Hospital Osijek, Osijek, Croatia, \\ ${ }^{4}$ Department of Neurology, General Hospital Dubrovnik, \\ Dubrovnik, Croatia, ${ }^{5}$ Research Department, Childrens \\ Hospital 'Srebrnjak', Zagreb, Croatia
}

Objective: The aims of this study were to assess the prevalence of migraine $(\mathrm{M})$, probable migraine (PM) and tension type headache (TTH) in the Croatian population, medical attendance and to report the degree of disability.

Methods: The design of the study was a cross-sectional survey using a self-completed questionnaire. The questionnaires were distributed to adults $>18$ years of age to contain a mix of urban, suburban and rural settings, and a spread of social class. Prevalence and demographic characteristics of headache types were analysed. Disability was measured using the Migraine Disability Assessment (MIDAS).

Results: Of the total 1542 responders, 640 (41,5\%) fullfiled the questionnaire indicating they suffer from headache. The proportion of females was $60,1 \%$ and males $38,1 \%$. The prevalence of M was 7,5\%, PM 11,5\%, M+PM 18,9\% and TTH 21,2\%. Patients with high level of education are more likely to have M or PM than TTH $(p=0.052)$. Marital status does not influence the presence of migraine or TTH $(\mathrm{p}=0.3)$, neither does the employment status $(\mathrm{p}=0.07)$. The prevalence of $\mathrm{M}$ and $\mathrm{PM}$ is higher in subjects living in cities $(\mathrm{p}<0,0001)$. Patients with $\mathrm{M}$ are more likely to visit a doctor regularly. More than a half of patients with TTH have never visited a doctor. Patients with PM have worse MIDAS assessment.

Conclusions: The prevalence of primary headaches is relatively high in Croatian population. Low percentage of patients searching for profesional help and poor MIDAS assessment indicates that the treatment of primary headaches should be improved in Croatia. 


\section{SS5/2}

\section{Prevalence of Chronic Daily Headaches and Socio Economic Factors in Countries of the Former Soviet Union}

\author{
Katsarava Z. ${ }^{1}$, Moldovanu I. ${ }^{2}$ \\ ${ }^{1}$ University of Essen, Essen, Germany, ${ }^{2}$ State Medical \\ University, Chishinau, Moldova
}

Objective: To estimate the 1-year prevalences of chronic daily headache $(\mathrm{CDH})$ and identify their principal risk factors in the Countries of former Soviet Union (e. g. Georgia and Moldova).

Methods: In a community-based door-to-door survey, medical residents interviewed pre defined numbers of biologically unrelated adults in the capitals and rural areas in Georgia and Moldova.

Results: The target populations were 1,145 respondents in Georgia and 1750 respondents in Moldova. The 1-year prevalence of CDH in Georgia was 7.6\% [95\% CI 6.0\%-9.1\%], in Moldova $6.8 \%$ [95\%CI 5.7\%-8.0\%]. Female gender, low socioeconomic status and frequent use ( $\geq 10$ days/month) of acute headache drugs were risk factors.

Conclusion: We present the first population based studies on headache from post Soviet Countries. In contrast to studies in Europe and USA, the prevalence of chronic headache in Post Soviet is remarkably high. The results further demonstrate the importance of socioeconomic factors in developing countries and unmask the unmet needs of people suffering from headache disorders.

\section{SS5/3 \\ Prevalence of Rare Trigeminal and Trigeminal Autonomic Headaches in a Population-Based Sample in Germany: A GHC Study}

\author{
Katsarava Z. ${ }^{1}$, Müller D. ${ }^{2}$, Yoon M.-S. ${ }^{2}$, Obermann M. ${ }^{2}$, \\ Dommes P. ${ }^{2}$, Diener H.-C. ${ }^{2}$ \\ ${ }^{1}$ University of Essen, Neurology, Essen, Germany, \\ ${ }^{2}$ University of Essen, Essen, Germany
}

Aim: We aimed to estimate the prevalence of the trigeminal neuralgia (TN), idiopathic facial pain (IFP) and trigeminal autonomic headaches (Cluster headache, $\mathrm{CH}$ ) in a population based sample in Germany.

Methods: The study was embedded into the large scaled population based study of the German Headache Consortium (GHC). In this part 6000 inhabitants of the city of Essen older than 18 years were screened by using a self assessing questionnaire. Three thoUSAnd three hundred and six people (56\%) responded. Of those $\mathrm{CH}$ was supscted in 182 subjects. 327 subjects reported having recurrent or daily facial pain. Both groups were by one of six neurologists.
Results: The diagnosis of $\mathrm{CH}$ was confirmed in 4 cases, of $\mathrm{TN}$ in 10 cases, of IFP in one case. The prevalence of $\mathrm{CH}$ was therefore was therefore estimated to be 119 per 100.000 [95\% CI $3-238$ per 100.000$]$, of TN $0.3 \%$ [95\% $0.1-0.5 \%$ ], the prevalence of PIFP $0.03 \%$ [95\% CI $<0.08 \%$ ].

Conclusion: Here we present the first data on the prevalence of rare trigeminal headaches in Germany.

\section{SS5/4 \\ Prevalence of Chronic Daily Headache, Chronic Migraine with and without Medication Overuse in a Population Based Sample in Germany}

\author{
Katsarava Z. ${ }^{1}$, Yoon M.-S. ${ }^{2}$, Obermann M. ${ }^{2}$, Dommes $P^{2}$ \\ Diener H.-C. ${ }^{2}$ \\ ${ }^{1}$ University of Essen, Neurology, Essen, Germany, \\ ${ }^{2}$ University of Essen, Essen, Germany
}

Objective/Methods: The German Headache Consortium (GHC) mailed questionnaires to a random sample of 15,705 18-65 year-olds residing in demographically diverse regions including a large (City of Essen) and midsize city (Münster) and a rural area (Sigmaringen) of Germany. Non-respondents were interviewed by telephone when possible. CHD was defined as $\geq 15$ headache days per month with or without medication overuse (intake of any kind of acute headache drugs on more than 15 days per month). We used three definitions of CM: CM_I ( $\geq 15 \mathrm{HA} /$ days a month with at least $50 \%$ of headaches being migraine or probable migraine days), CM_II ( $\geq 15$ HA days/month with at least 8 migraine or probable migraine days), CM_III ( $\geq 15$ HA days/month with reported any migraine or probable migraine days).

Results: There were 9,968 respondents (response rate $=63.4 \%$ ). The prevalence of $\mathrm{CDH}$ was $\mathrm{CH} 2.8 \%$; of those $12 \%$ overused the acute headache drugs. CM_I definition was the most restrictive (prevalence $0.4 \% ; \mathrm{MO}=27 \%$ ) followed by CM_II (prevalence 0.5\%; MO = 31\%), CM_III (prevalence $1.9 \% ; \overline{\mathrm{MO}}=11 \%$ ). Epidemiologically all groups were fairly similar: females $(\mathrm{CM} \mathrm{I}=70 \%$; CM II $=69 \%$; CM III $=71 \%$; $\mathrm{CDH}=64 \%)$, mean age: $44-46$ years $\overline{(} \pm 14)$, mean BMI $25.3-26.5$, low (non high school) education $(\mathrm{CM} I \mathrm{I}=70 \%$; CM_II $=73 \%$; CM_III $=78 \%$; $\mathrm{CDH}=72 \%$ ).

Conclusions: The prevalence of $\mathrm{CDH}$ in Germany is in line with the reports from other European countries and US. CM prevalence varies by case definition, whereby CM_I is a subset of CM II which in turn is a subset of CM III and all are subsets of $\mathrm{CD} \overline{\mathrm{H}}$. Demographic similarities among the definitions suggest these groups may overlap biologically. 
SS6

Stroke Epidemiology and Management (Part 2)

August 29, 2009

14:00-15:00

SS6/1

Proportion, Risk Factors, and Outcome of Lacunar Infarct in Chinese: A Hospital-Based Study

Wu B., LiU M.

Department of Neurology, West China Hospital, Sichuan University, Chengdu, China

Background and Purpose: The aim of this study was to investigate the proportion, risk factors, case fatality, and functional outcome of a first-ever lacunar infarct and to determine the relevant prognostic variables.

Methods: Prospective registry data entered within one month after a first-ever stroke between March 2002 and March 2007 were used from 4 Chinese hospitals. Data regarding stroke risk factors and baseline characteristics were examined in a crosssectional long-term follow-up. Case fatality, death or dependency, and stroke recurrence were assessed at 30, 90, 180 days, and one year. Predictive factors for death at 1-year in patients with lacunar infarct were tested by logistic regression analysis.

Results: A total of 3905 patients with first-ever cerebral infarct were included, of whom 1650 (42.3\%) had a lacunar infarct and $2255(57.7 \%)$ a non-lacunar infarct. Case fatality was lower in patients with lacunar infarct at all four time points $(5.3$, $6.4,7.8$, and $17.4 \%$, respectively), as compared to patients with non-lacunar infarct $(9.5,14.3,16.5$, and $20.1 \%$, respectively). Age, diabetes, smoking, and recurrent stroke were the independent predictors for 1-year case fatality in patients with lacunar infarct. Death or dependency was also lower in lacunar infarct patients than in non-lacunar infarct patients.

Conclusions: The proportion of lacunar infarct in China was much higher than that of in western countries. The long-term prognosis of lacunar infarct is not benign. Secondary prevention of lacunar infarct is the most important measure for decreasing death or dependency in the long term.
SS6/2

\section{The Austrian Acute Stroke Registry for Monitoring Stroke Units Shows High Efficiency for Short Transport Times and High Thrombolysis Rates}

\author{
Eckhardt R. ${ }^{1}$, Lang W. ${ }^{2}$, Tatschl C. ${ }^{1}$, Ferrari J. ${ }^{2}$, Hofer C. ${ }^{3}$ \\ Brainin M. ${ }^{4}$, Austrian Stroke Unit Registry Study Group \\ ${ }^{1}$ Landesklinikum Donauregion Tulln, Neurology, Tulln, \\ Austria, ${ }^{2}$ Hospital Barmherzige Brueder, Neurology, \\ Vienna, Austria, ${ }^{3} \mathrm{GÖG}$ - Gesundheit Österreich $\mathrm{GmbH}$, \\ Vienna, Austria, ${ }^{4}$ Danube University Krems, Center of \\ Clinical Neurosciences, Krems, Austria
}

In Austria, acute stroke units have been set up within the last decade following evidence-based principles of time constraints ('Time is Brain'). Transport times to all units are now less than 45 minutes from most regions in Austria, with the exception of very few mountain regions. Stroke units have uniform standardized equipment including CT (and most also MRI), neurosonography, laboratory and monitoring of vital parameters.

For concomitant assessment of efficiency and quality control a multicenter online registry has been set up as collaboration between the Austrian Society for Stroke Research and the National Research and Planning Institute for Health Care (Gesundheit Österreich $\mathrm{GmbH})$. This acute stroke registry involves most of the 34 stroke units and now covers more than $60 \%$ of all hospital admissions for stroke. It includes a 3 month follow-up. Evaluating 32.000 stroke patients assessed between January 2003 and April 2009 it shows a high level of efficiency in all monitored parameters including short transport times, access to early diagnosis and imaging. The overall thrombolysis rate is increasingly high, in the year 2008 even more than $13 \%$. When patients are selected that are eligible for thrombolysis according to licensed criteria (age under 80 years, etiology ischemic stroke, NIHSS more than 3 points, arrival within 120 minutes,) the thrombolysis rate now exceeds $50 \%$. Thus, such a system of competence can be considered suitable for managing the time constraints of acute stroke care and to give the best possible chances for monitoring efficiency and quality issues over time.

SS6/3

A Progress in Stroke Prevention: The Decrease in the Incidence of Stroke from Atrial Fibrillation, in Dijon, for 20 Years

\section{Bejot Y., Jacquin A., Osseby G.-V., Durier J., Rouaud O., Giroud M. \\ University of Burgondy, Neurology and Epidemiology, Dijon, France}

Background: Epidemiological studies are needed to evaluate the impact of the recent changes in clinical practice on the incidence of cardioembolic stroke with atrial fibrillation (CE/AF stroke).

Methods: Overall ischemic strokes were prospectively ascertained in the population-based registry of Dijon, France, from 
1985 to 2006 . We evaluated trends in incidence rates, risk factors and prestroke therapy in CE/AF stroke.

Results: 3064 ischemic strokes including 572 (18.7\%) CE/ AF strokes were recorded. Over the 22 years, a decrease in the incidence of overall CE/AF stroke was noted ([IRR] 0.9858, 95\% CI $0.9731-0.9986 ; \mathrm{p}=0.03)$. We observed a higher prevalence of previous AF, previous myocardial infarction, and patients aged $>70$ years in CE/AF stroke $(\mathrm{p}<0.0001)$ whereas hypercholesterolemia was more prevalent in other ischemic strokes $(p=0.003)$. A significant increase in the use of anticoagulants and antiplatelet agents was noted, and was particularly pronounced for $\mathrm{CE} / \mathrm{AF}$ stroke with previous AF.

Conclusions: The decrease in the incidence of $\mathrm{CE} / \mathrm{AF}$ stroke may reflect the increase in the utilization of antithrombotic therapy in patients with AF, but the use of such therapies will have to increase further because of the expected ageing of the population in coming years.

\section{SS6/4}

\section{Individual Stroke Subtype Classifications Match with the "Causative Classification of Stroke System (CCSS)' and the 'A-S-C-O Classification'}

\author{
Wolf M., Szabo K., Förster A., Kern R., Hennerici M.
}

Department of Neurology, Universitätsmedizin Mannheim, University of Heidelberg, Mannheim, Germany

Background: Precise analysis of stroke subtypes is of clinical and epidemiological interest. Many classifications of stroke subtypes exist, but considering interests, the gain of knowledge and new diagnostic techniques, some have become outdated. Ay et al. developed a revised internet accessible TOAST classification (CCSS), furthermore, a new phenotypic classification (ASCO) has been proposed by Amarenco et al. and needs further evaluation.

Methods: We assigned 106 patients from our stroke data base, analysed data and compared our classification (MA) with the CCSS and ASCO. Subjects were randomly selected into three groups $(\mathrm{NIH} \leq 3, \mathrm{NIH} \geq 4, \mathrm{NIH} \geq 4$ with intravenous thrombolysis). Clinical features and complementary investigations were recorded according to a standardized acute stroke care protocol.

Results: General concordance between MA and CCSS evaluation was high (83\%), especially for large vessel disease $(93.75 \%)$ and cardio-embolic mechanisms (97.44\%). Analysis of subgroups showed concordance of $74 \%$ for $\mathrm{NIH} \leq 3,85 \%$ for $\mathrm{NIH} \geq 4$ and $91 \%$ for $\mathrm{NIH} \geq 4$ with thrombolysis. Most discordances appeared for DWI negative TIAs, classified as small vessel in MA but remaining undetermined with the CCSS. Comparing data with the ASCO, few discordances were found, about half of the patients got classified with more than one etiologic category.

Discussion: Concordance between evaluation systems was high, however there are considerable differences concerning TIA patients with DWI negative MRI between MA and CCSS. ASCO had a high concordance with our results and has a more comprehensive character than previous classifications especially in cases of concomitant etiologies.

\section{SS6/5 \\ Differences in Stroke Incidence Among City Health Districts: Could They Be a Tool to Evaluate Primary Prevention?}

\author{
Cabral N. ${ }^{1}$, Eluf-Neto J. ${ }^{2}$, Gonçalves A. ${ }^{1}$, Longo A. ${ }^{1}$, \\ Moro C. ${ }^{1}$, Ferst P. ${ }^{1}$, Oliveira ${ }^{3}{ }^{3}$, Vieira ${ }^{3}{ }^{3}$, Fonseca L.A.M. ${ }^{4}$ \\ ${ }^{1}$ University of the Region of Joinville-Univille, Joinville, \\ Brazil, ${ }^{2}$ Department of Preventive Medicine Faculdade de \\ Medicina da Universidade de Sao Paulo, Sao Paulo, Brazil, \\ ${ }^{3}$ Department of Geography of University of Region of \\ Joinville-Univille, Joinville, Brazil, ${ }^{4}$ Department of \\ Preventive Medicine Faculdade de Medicina da \\ Universidade de Sao Paulo, Joinville, Brazil
}

Background: In Joinville, Brazil, stroke incidence rate (SIR) in 2005-6 was 105.4 per $10^{5}$, which is similar to the rates found in developed countries. Current evidences suggest an association between socioeconomic status and stroke incidence. As Joinville and Brazil as a whole both present social inequalities and also geographic differences in SIR, a potential for primary prevention may exist. Thus, our aim was to measure the SIR on each of the city's nine health districts (CHD) in order to assess whether there are gradients in rates among them.

Methods: We prospectively ascertained all possible stroke cases occurring on the city of Joinville during 2005-2007. We determined age-adjusted incidence for each of the nine CHD, as well as their mean income, years of education, mean age and the proportion of the district's covered by a governmental primary health care program (GPHP).

Results: Of the 1734 stroke cases registered, 1034 were firstever strokes. The Joinville crude SIR per $10^{5}$ was 69.5 (95\% CI, 65.3-73.9). The SIR among CHD ranged from 43 to 85 per $10 .^{5}$ A multiple linear regression analysis with all variables included showed that SIR differences were only correlated with years of education $(R=0.839, p=0,02)$.

Conclusions: Years of education can explain a wide SIR variation among districts. By the other hand, GPHP not seem to influence SIR. These results may be useful to guide the allocation of the resources in primary prevention politics. 


\section{SS6/6 \\ Acute Case-Fatality of First-Ever Stroke and Related Pre-Hospital Predictors: Results from Takashima Stroke Registry, Japan (1988-2002)}

Turin T. ${ }^{1}$, Kita Y. ${ }^{1}$, Rumana N. ${ }^{1}$, Nakamura Y. ${ }^{2}$, Takashima N. ${ }^{1}$, Ichikawa M. ${ }^{3}$, Sugihara $H^{3}{ }^{3}$, Morita $Y^{4}$, Hirose $K^{5}$,

Okayama A. ${ }^{6}$, Miura K. ${ }^{1}$, Ueshima $H^{1}{ }^{1}$

${ }^{1}$ Department of Health Science, Shiga University of Medical Science, Otsu City, Japan, ${ }^{2}$ Kyoto Women's University, Kyoto, Japan, ${ }^{3}$ Takashima General Hospital, Takashima, Shiga, Japan, ${ }^{4}$ Makino Hospital, Takashima, Shiga, Japan, ${ }^{5}$ Otsu Red Cross Hospital, Otsu City, Shiga, Japan, ${ }^{6}$ The First Inst. for Health Promotion and Health Care, Tokyo, Japan

Objective: Few population based studies have evaluated the factors influencing or predicting acute case-fatality of stroke in an unselected series of patients. The purpose of this study was to define predictors of case-fatality within 28-days after a first-ever stroke for stroke subtypes of cerebral infarction (CI) and cerebral hemorrhage $(\mathrm{CH})$.

Methods: Between 1988 and 2002, all individuals with a suspected acute stroke who were resident in a geographically defined region of Takashima County, Shiga prefecture, Japan, with a population of $\approx 55,000$ people, were registered prospectively and assessed according to standardized diagnostic criteria. The 28 -day case-fatality rates and $95 \%$ confidence intervals $(95 \% \mathrm{CI})$ were calculated considering the day of onset as day zero. In multivariate analyses, the influence of age, gender, co-morbidities, and neurological deficits at admission on risk of acute fatality was investigated.

Results: There were 1177 registered first-ever CI patients (men:665 and women:512) and 380 registered first-ever $\mathrm{CH}$ patients (men:188 and women:192) during the study period. The 28-day case-fatality was $22.6 \%(86 / 380)$ for $\mathrm{CH}$ and $7.4 \%(87 / 1177)$ for CI. Multivariate analysis revealed that initial level of consciousness at admission, hematoma volume, and admission after 3 hours of onset was independent predictors of death at 28-days for $\mathrm{CH}$. For CI, previous history of TIA, levels of consciousness at admission were independent predictors of acute fatality.

Conclusion: $\mathrm{CH}$ remains a stroke subtype associated with a higher fatality than CI. For both subtypes the level of consciousness on admission is the strongest predictor of acute fatal outcome.

\section{SS7}

Epilepsy and Other Seizure/ Neurological Disorders Epidemiology (Part 1)

August 29, 2009

14:00-15:00

\section{SS7/1 \\ First Stroke and Acute Symptomatic Seizures: A Prospective Cohort Study. The Epistroke Group}

\begin{abstract}
Beghi E. ${ }^{1}, D^{\prime}$ Alessandro R. ${ }^{2}$, Consoli D. ${ }^{3}$, Crespi V. ${ }^{4}$ Delaj L. ${ }^{2}$, Gandolfo C. ${ }^{5}$, Greco G. ${ }^{6}$, Manfredi M. ${ }^{7}$, Mattana F. ${ }^{1}$ Musolino R. ${ }^{8}$, Provinciali L. ${ }^{9}$, Santangelo M. ${ }^{6}$, Specchio L. ${ }^{10}$, EPISTROKE Group

'Istituto di Ricerche Farmacologiche 'Mario Negri', Milano, Italy, ${ }^{2}$ Dipartimento di Scienze Neurologiche, Unità di Neuroepidemiologia, Bologna, Bologna, Italy, ${ }^{3}$ U.O. di Neurologia, A.S. Ospedale 'G.Jazzolino', Vibo Valentia, Italy, ${ }^{4}$ U.O. di Neurologia, A.O. 'Ospedale Civile', Vimercate, Italy, ${ }^{5}$ Dipartimento di Neuroscienze, Oftalmologia e Genetica, Centro Ictus, Genova, Italy, 'Unità Operativa di Neurologia, 'Ospedale Ramazzini', Carpi, Italy, ${ }^{7}$ Clinica Neurologica, Università 'La Sapienza', Roma, Italy, ${ }^{8}$ U.O.C. di Clinica Neurologica, Policlinico Universitario, Messina, Italy, ${ }^{9}$ Clinica di Neurologia, Università Politecnica delle Marche, Ancona, Italy, ${ }^{10} \mathrm{Clinica}$ delle Malattie del Sistema Nervoso, A.O. Universitaria Ospedali Riuniti, Foggia, Italy
\end{abstract}

Background: Acute symptomatic seizures (ASS) have been reported in $2-33 \%$ of patients with stroke. Differing populations and methods of case ascertainment are possible explanations.

Objectives: To assess incidence and predictors of ASS in a prospective cohort of patients with first stroke.

Patients and Methods: Patients hospitalized in 32 Italian centers were recruited. Demographic data, disease characteristics and risk factors were collected. ASS ( $\leq 7$ days) were recorded and correlated to age, gender, family history of epilepsy, lesion type, site and size.

Results: 639 patients (278 women and 361 men aged 30 to 97 years) were enrolled. $542(84.8 \%)$ had ischemic stroke (32 with hemorrhagic infarction) and $97(15.2 \%)$ had hemorrhagic stroke. 123 patients $(19.2 \%)$ had a large lesion $(>3 \mathrm{~cm})$ and $263(41.1 \%)$ a cortical lesion. 39 patients $(6.1 \%)$ presented ASS, 19 with cerebral infarction (3.8\%), 4 with hemorrhagic infarction (12.5\%), and 15 $(15.5 \%)$ with cerebral hemorrhage. The relative risk of seizures was 1.51 (95\% CI 1.1-2.0) with hemorrhagic stroke, $4.46(95 \%$ CI 1.2-16.4) with a large lesion, and 1.64 (95\% CI 1.1-12.5) with a cortical lesion. The risk with hemorrhagic stroke was $4.01(95 \%$ CI 1.1-14.3) after adjusting for age, gender, lesion size and site, and family history of epilepsy. The adjusted risk of Rankin $>2$ at one month in patients with ASS was 1.05 (95\% CI 1.0 - 1.1). 
Conclusions: The incidence of ASS is low in patients with first stroke. Type of stroke is the only independent predictor. ASS may be a prognostic factor of greater disability.

\section{$\mathrm{SS7/2}$ \\ Incidence and Mortality Rates of Epilepsy in the City of Kolkata, India}

\author{
Banerjee T.K. ${ }^{1}$, Das S.K. ${ }^{2}$, Roy $T^{2}{ }^{2}$, Roy B. ${ }^{2}$, Jena S. ${ }^{2}$, \\ Hazra A. ${ }^{3}$, Chaudhuri A. ${ }^{4}$ \\ ${ }^{1}$ Department of Neurology, National Neurosciences Centre \\ Calcutta, Kolkata, India, ${ }^{2}$ Department of Neurology, \\ Bangur Institute of Neurosciences and Psychiatry, Kolkata, \\ India, ${ }^{3}$ Department of Pharmacology, Institute of Post- \\ graduate Medical Education \& Research, Kolkata, India, \\ ${ }^{4}$ Indian Statistical Institute, Kolkata, India
}

Aim: There is a dearth of prospective studies on epilepsy in India. To our knowledge, this was the first urban Indian study on Incidence and Mortality of Epilepsy.

Methods: This was a longitudinal descriptive study comprising 2-stage door-to-door survey of a stratified randomly selected sample population of the city of Kolkata, the eastern metropolis of India. The survey was conducted annually for consecutive 5 years from 2003 through 2008.

Results: The population surveyed was 52,377 (27,626 men, 24,751 women). There were 64 new cases of epilepsy. The average incidence rate of epilepsy is 24.44 (95\% CI, 22.3-26.7) per 100,000 persons per year. When age-standardized to world standard population, the incidence rate is 26.40 (95\% CI, 24.1-28.8) per 100,000 persons per year. The age-specific incidence rate of epilepsy shows bimodal distribution. During the 5-year period of study, 18 cases of epilepsy died. The mortality rate of active epilepsy, age-standardized to Indian general population is 17.74 (95\% CI, 16.2-19.4) per 1000 person-years. The standardized mortality ratios (SMR) are 2.8 and 3.1 compared to Indian urban and Kolkata population, respectively.

Conclusion: The age-standardized incidence and mortality rates of epilepsy in our study are similar to that observed in the western nations.

\section{SS7/3 \\ History of the Development of Neuroepidemiology in the People's Republic of China}

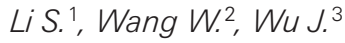 \\ ${ }^{1}$ China Association against Epilepsy, Beijing, China, \\ ${ }^{2}$ Beijing Neurosurgical Institute, Neuroepidemiology, \\ Beijing, China, ${ }^{3}$ Beijing Neurosurgical Institute, Beijing, \\ China
}

Neuroepidemiology was introduced to China in early 1980's by Dr. Bruce Schoenberg, NINCDS, NIH, United States. First pilot door-to-door survey on prevalence of neurological diseases was conducted in 1981 in urban Beijing. Extended surveys in 6 cities and rural area of 22 provinces followed and thus get ting the first group of data on morbidity and mortality of neurological diseases in China were figured out. From 1986 to 1995, an intervention trial on stroke prevention and control was carried out and good results reported. At the same time, a feasibility test of community control for epilepsy led by WHO was conducted.

In the 21 century, under the coordination of the Global Campaign against Epilepsy (GCAE) by ILAE/IBE/WHO, a demonstration project was implemented in rural area of 6 provinces. Using a fixed protocol, the trained township medical workers to treat and follow-up convulsive epileptic patients with phenobarbital, and good results reported. Then an extensive project on epilepsy control in rural area has been implementing till now, which covered around 40 million population and 30,000 patients with convulsive epilepsy were under treatment. Presently, a followup study for the GCAE demonstration project and the impact on cognition by phenobarbital are being carried out. Some concrete figures from those studies are reported in the presentation.

\section{SS7/4}

\section{2-24 Year Mortality Data from the National General Practice Study of Epilepsy (NGPSE)}

\author{
Neligan A., Bell G.S., Shorvon S.D., Sander J.W. \\ Department of Clinical and Experimental Epilepsy, UCL \\ Institute of Neurology, London, United Kingdom
}

Background: The National General Practice Study of Epilepsy (NGPSE) was set up in 1984 and is one of the longest established epilepsy community-based studies in existence. The study has followed up 792 patients with definite or possible epilepsy and 220 patients with febrile seizures who were enrolled by their General Practitioners (GPs). Mortality data in the cohort were presented in 19941 and 2001.

Methods: A long-term prospective community based study of an incident cohort of patients diagnosed with epilepsy or febrile convulsions in the early 1980s.

Results: At seven years of follow-up 150 deaths had occurred in those with definite or possible epilepsy and the Standardised Mortality Ratio (SMR) was 2.5 (95\% CI 2.1 to 2.9). The SMR was highest in the first year after presentation (SMR 5). At 14 
years of follow-up there were 199 deaths in this cohort and the SMR was 2.1 (95\% CI 1.8 to 2.4). Current follow-up, after 24 years, shows that 290 people have died; additionally, two who presented with febrile seizures have also died. On primary analysis, the most frequent causes of death included pneumonia, cancer (eg brain, breast, lung, and bladder), myocardial infarction and stroke. There were few reported epilepsy-related deaths - a further one in addition to the five already reported.

Conclusions: This study continues to confirm the increased premature mortality in people with epilepsy. The community basis of the study is important to put this risk into perspective as this is often exaggerated in hospital-based studies.

\section{LB-SS/SS7/5 \\ Automated On-Line Monitoring of Health- Related Quality of Life and Fatigue in Relapsing Remitting Multiple Sclerosis Patients Treated with Glatiramer Acetate (Optivit Study)}

\author{
Jongen P.J.H., Sanders E., Visser L., Zwanikken C., \\ Peters G., van der Tol M., Koopmans P., van Noort E., \\ OPTIVIT Study Group \\ MS4 Research Institute, Nijmegen, Netherlands
}

Objectives: To study by repeated automated on-line assessments health-related quality of life (HR-QoL), fatigue and treatment satisfaction in relapsing remitting multiple sclerosis (RRMS) patients in the first year of glatiramer acetate (GA) (Copaxone $\left.{ }^{\circledR}\right)$ treatment.

Methods: Patient-oriented, observational, prospective, multicentre study in the Netherlands. Automated assessments at baseline, month 6 (M6) and month12 (M12) by an on-line program using short validated questionnaires - Leeds Quality of Life Scale (LQoLS), Modified Fatigue Impact Scale (MFIS) -, and for treatment satisfaction a Visual Analogue Scale (VAS). Pre-defined subgroup analyses of interferon-beta (INF-beta) pre-treated and treatment-naïve patients with intra-group and without inter-group comparisons. Mean values with confidence interval (CI) or standard deviation (SD) are given.

Results: Patients. 43 neurologists included 176 patients. 167 patients were analysed, 86 treatment-naïve and 81 INF-beta pre-treated. Disease duration was 5.2, 3.8 and 6.7 yrs., Expanded Disability Status Scale score 2.8, 2.1 and 3.6, and annualised relapse rate $1.1,0.9$ and 1.3 , respectively. 130 (78\%) patients completed both the two questionnaires and the VAS at M0, M6 and M12, irrespective of GA continuation. At M12 29 (17\%) patients had discontinued GA. LQoLS. LQoL scores increased from 19.6 at M0 (90\% CI: $19.0-20.2)$ to 21.7 at M6 (90\% CI: $21.1-22.3)$ and 22.2 at M12 (90\% CI: 21.6-22.8) (F 1,135 = 55.8, p<0.001). MFIS. MFIS scores decreased from 12.0 at M0 (90\%CI: $11.4-$ 12.6) to 10.9 at M6 (90\% CI: $10.3-11.5)$ and 10.2 at M12 (90\% CI: 9.5-10.8) (F 1,135 = 22.7, p<0.001). INF-beta pre-treatment was related to higher MFIS score $(\mathrm{p}=0.005)$. Treatment satisfaction. VAS score at M0 was 6.5 (SD 2.4), at M6 7.0 (SD 2.0) and at $\mathrm{M} 127.0$ (SD 2.2) $(\mathrm{F} 1,129=4.4, \mathrm{p}=0.038)$.
Conclusion: Six-monthly automated on-line assessments had a high complete response of $78 \%$ and showed $13 \%$ increase of HR-QoL and 15\% decrease of fatigue. We conclude that in RRMS patients in their first year of GA treatment changes in HR-QoL and fatigue as documented by automated on-line assessment are significant and comparable to those obtained by paper-and-pencil evaluations. Automated on-line monitoring may be an informative and patient-friendly tool for evaluating therapeutic aspects of immunomodulation.

Study was funded by Teva Pharma Nederland and Sanofiaventis Netherlands B.V.

\section{LB-SS/SS7/6 \\ The Spectrum of Demyelinating Inflammatory Idiopathic Diseases in the Southeast Region of Brazil}

Papais-Alvarenga R.M. ${ }^{1}$, Vasconcelos C. ${ }^{1}$, Alvarenga M. ${ }^{2}$, Brum D. ${ }^{3}$, Barreira A. ${ }^{4}$, Camargo S. ${ }^{5}$, Papais-Alvarenga M. ${ }^{1}$, Grupo de neuroimunologia da Academia Brasileira de Neuologia

${ }^{1}$ UNIRIO, Neurology, Rio de Janeiro, Brazil, ${ }^{2}$ Hospital Carlos Haya, Neurology, Malaga, Spain, ${ }^{3}$ Ribeirão Preto, Neurology, Ribeirão Preto, Brazil, ${ }^{4}$ USP - Ribeirão Preto, Neurology, Ribeirão Preto, Brazil, ${ }^{5}$ Hospital da Lagoa, Neurology, Rio de Janeiro, Brazil

Background: The spectrum of the idiopathic inflammatory demyelinating diseases of the CNS was amplified at the last decade. A substantial change in the MS diagnostic occurred with the inclusion of specific MRI criteria. A recurrent form of NMO was recognized. Brazil is a tropical country with a great ethnic diversity. The first MS Brazilian survey (South Atlantic project-1997) analyzed the clinical course of MS in patients classified by Poser et al (1983) in the categories clinical definite or clinical probable.

Objective: To review the diagnosis of MS after 10 years.

Methods: The medical records of $256 \mathrm{MS}$ patients from Rio de Janeiro (Hospital da Lagoa, UNIRIO, Clinica Bambina) or São Paulo (USP- Ribeirão Preto) were reviewed. Only patients on treatment were selected for revision and classified by clinical, MRI and CSF data in the spectrum of IIDD (2008).

Results: 220 patients were analyzed (RJ- 189; SP-31). IIDD was confirmed in $96 \%$. The diagnosis was changed in $4 \%$ - Arnold Chiari (1), HAM-TSP (2) lacunar stroke (3) spinal cord ischemia (1) convertion (2). The spectrum was MSRR (130), NMO (37), EMPP (26), ADEM (9), ATM (4), NO (3), pseudotumor (2).

Conclusion: The Poser criteria did not allow differential diagnosis among MS and other IIDD; 73,3\% of the Brazilian patients remained after 10 years with the MS diagnosis; 20,8\% had NMO or related conditions. All MS epidemiological studies in tropical areas must be revised to apply both criteria (MS and NMO). 
SS8

Stroke Epidemiology and Management (Part 3)

August 29, 2009

15:00-16:00

$\mathrm{SS} 8 / 1$

\section{Ischaemic Stroke Outcomes in Patients with Atrial Fibrillation: An Analysis of Linked Health and Mortality Data from an Australian Population}

\author{
Gattellari M. ${ }^{1}$, Worthington J. ${ }^{2}$, Aitken R. ${ }^{3}$, Jalaludin B. ${ }^{1}$
}

${ }^{1}$ The University of New South Wales, School of Public Health and Community Medicine, Sydney, Australia, ${ }^{2}$ The University of New South Wales, South West Sydney Clinical School, Sydney, Australia, ${ }^{3}$ NSW Health, Sydney, Australia

Background: Few studies have reported outcomes for stroke and atrial fibrillation (AF). We aimed to determine mortality of patients with AF up to 12-months after an ischaemic stroke.

Methods: We identified patients separated from hospital with a primary diagnosis of ischaemic stroke (ICD-10, I63.x) from July 2000 to June 2006 within New South Wales, Australia (population $\sim 6.5$ million). Deaths were ascertained using the state death registry.

Results: Of the 25,806 patients with ischaemic stroke, $23.8 \%(n=6136)$ had a recorded diagnosis of AF. Patients with AF were older (Mean $=78.5$ vs 72.2 years), more likely to be female $(56.2 \%$ vs $45.6 \%)$ and had higher Charlson Comorbidity scores $($ Mean $=2.2$ vs 1.7$)$. Patients with AF were more likely to develop pneumonia and receive intensive care during hospitalisation (17.8\% vs $7.9 \%$ and $8.1 \%$ vs $4.5 \%$, respectively) $(\mathrm{p}<$ 0.001 ) and to have longer hospital stays (Median $=20$ vs 12.2 days) $\left(\mathrm{P}^{\prime} \mathrm{s}<0.001\right)$

Crude mortality at 30-days, 90-days and 12-months was $19.4 \%, 27.9 \%$ and $38.7 \%$, respectively, compared with $11.1 \%$, $15.4 \%$ and $22.3 \%$ in patients without $\mathrm{AF}\left(\mathrm{P}^{\prime} \mathrm{s}<0.001\right)$. Almost one-fifth (17.5\%) of patients with AF younger than 65 died within 12 -months while $55.9 \%$ of those over 85 had died. In 30-day survivors, $23.9 \%$ with $\mathrm{AF}$ had died within 12-months compared with $12.6 \%$ of patients without $\mathrm{AF}$ ( $\mathrm{p}<0.001)$. When adjusting for age, sex, Charlson score and year of separation, AF conferred a 1.55 greater odds of death at 12 -months $(95 \% \mathrm{CI}=1.45-1.66)$.

Conclusion: Patients with AF have a high risk of death in the year following ischaemic stroke.
SS8/2

\section{Patient Dissatisfaction with Acute Stroke Care. A Nationwide Analysis}

\author{
Asplund K. ${ }^{1}$, Jonsson F. ${ }^{1}$, Eriksson M. ${ }^{1}$, Stegmayr B. ${ }^{2}$, \\ Appelros P. ${ }^{3}$, Norrving B. ${ }^{4}$, Terént A. ${ }^{5}$, Hulter Asberg K. ${ }^{6}$, \\ The Riks-Stroke Collaboration \\ ${ }^{1}$ Riks-Stroke, University Hospital, Umea, Sweden, \\ ${ }^{2}$ National Board of Health and Welfare, Epidemiological \\ Center, Stockholm, Sweden, ${ }^{3}$ Department of Neurology, \\ University Hospital, Orebro, Sweden, ${ }^{4}$ Department of \\ Neurology, Lund University Hospital, Lund, Sweden, \\ ${ }^{5}$ Akademiska University Hospital, Department of \\ Medicine, Uppsala, Sweden, ${ }^{6}$ Department of Medicine, \\ Enkoping Hospital, Enkoping, Sweden
}

Background: Riks-Stroke, the Swedish Stroke Register, was used to explore the determinants of patient dissatisfaction with acute in-hospital care.

Methods: All 79 hospitals in Sweden admitting acute stroke patients participate in Riks-Stroke. During 2001-2007, 104,876 patients ( $87 \%$ of survivors) responded to a follow-up 3 months after acute stroke. In univariate and multivariate analyses, dissatisfaction with acute stroke care was related to stroke care and patient characteristics.

Results: The proportion of patients being dissatisfied with acute stroke care declined over the years 2001-2007. It was more common in women than in men and closely associated with severity of stroke at onset, functional outcome at 3 months and with poor self-perceived general health and feeling of being depressed. Dissatisfaction with acute stroke care was closely linked to dissatisfaction with other aspects of care, including rehabilitation and support by community services. After adjustment for case-mix, the proportion of dissatisfied patients was significantly higher in large compared to small hospitals. Patients treated in stroke units were less often dissatisfied than patients in general wards, as were patients who had participated in discharge planning and who had an out-patient follow-up visit.

Conclusions: Dissatisfaction with in-hospital acute stroke care is part of a more extensive complex comprising poor functional outcome, depressive mood, poor self-conceived general health and dissatisfaction not only with acute care but also with healthcare and social services at large. Several aspects of stroke care organization, including access to care in stroke units, may alleviate the risk of dissatisfaction. 


\section{SS8/3 \\ Sex-Specific Differences in 1-Week to 1-Year Survival After Stroke in Post-Menopausal Women and Men of Similar Age}

Olsen T.S. ${ }^{1}$, Andersen K.K. ${ }^{2}$

${ }^{1}$ Hvidovre University Hospital, The Stroke Unit, Hvidovre, Denmark, ${ }^{2}$ Technical University of Denmark, Informatics and Mathematical Modeling, Section for Statistics, Lyngby, Denmark

Background: Hormonal factors especially estrogens are thought to be related to the gender gap in longevity. We studied sex-specific differences in survival after stroke in post-menopaUSAl women (age $>52$ years) and men of similar age.

Method: A registry was started 2001 aiming to register all hospitalized stroke patients in Denmark. Complete information on risk factors, stroke severity (Scandinavian Stroke Scale (SSS), 0-58) and CT-scan were obtained in 25607 patients. Survival was followed up to one year from stroke. Curves of 1-week, 1-month, 3 -months and 1-year survival in men and women related to age, with fixed stroke severity scores were established.

Results: 1-week, 1-month, 3-months and 1-year survival adjusted for stroke severity was higher in women throughout the entire life span. The female survival advantage increased significantly with increasing age. At the menopause (age 52 years; SSS fixed at 12.) the female survival advantage was $15 \%$ (1-week survival), 13\% (1-month survival) and 6\% (3-months survival and 1 -year survival). At the age of $60 / 70 / 80$ years the survival advantage had increased by factor $2.0 / 3.1 / 5.0$ (1-week survival), 1.7 / $3.1 / 4.7$ (1-month survival), 2.2 / 4.2 / 5.1 (3-months survival) and 1.7 / 2.5 / 2.8 (1-year survival).

Conclusion: The female survival advantage continues after the menopause and increases with age especially as regards to early survival (1-week to 3-months) suggesting an effect especially on stroke-lesion related mortality.

\section{SS8/4 \\ Patients with Acute Ischemic Stroke Are Arriving Later to Emergency Departments After Symptom Onset}

\author{
Kleindorfer D. ', Khoury J. ${ }^{2}$, Alwell K. 1, Moomaw C. ${ }^{1}$, \\ Woo D. ', Flaherty M. ', Khatri P. ${ }^{1}$, Adeoye O. ${ }^{3}$, Ferioli S. ${ }^{1}$, \\ Kissela $B^{1}$ \\ ${ }^{1}$ University of Cincinnati, Neurology, Cincinnati, USA, \\ ${ }^{2}$ Cincinnati Children's Hospital, Cincinnati, USA, ${ }^{3}$ University \\ of Cincinnati, Emergency Medicine, Cincinnati, USA
}

Background: The most important reason acute ischemic stroke (IS) patients are not given thrombolytic therapy is delay in presenting to medical attention. Previously we reported a marginal improvement between 1993/94 and 1999 in how quickly patients arrive to the emergency department after stroke symptom onset. We hypothesized that recent nationwide efforts to improve stroke awareness and to certify stroke centers would further improve arrival times in 2005 within our large, biracial population.

Methods: Using ICD-9 codes 430-436, we ascertained all IS events that presented to a local emergency department within our population of 1.3 million in 7/93-6/94, 1999, and 2005. Comparisons were made between study periods, and by race and gender using chi square.

Results: There were 1,792 IS patients who presented to an ED in 1993/94, 1,973 in 1999, and 1,787 in 2005. Documentation of exact onset times improved between 1993/94 and 1999 (38\% vs. 42\%) but worsened in 2005 (34\%). The percentage of patients arriving in under 3 hours improved between 1993/94 vs. 1999 ( $23 \%$ vs. $26 \%, p=0.03)$, but worsened in $2005(23 \%, p=0.008)$. Blacks and women were less likely to arrive in less than three hours than whites and men, respectively, in 2005, and there was a significant race-sex interaction $(\mathrm{p}=0.03)$.

Conclusions: Nationwide efforts to improve public awareness regarding stroke were not reflected in arrival times after symptom onset. Few patients arrive in the 3-8 hour window, hampering attempts to lengthen the acute treatment time window.

\section{SS8/5 \\ Do Multiple Prior Transient Ischemic Attacks Affect Short-Term Risk of Stroke? \\ Kleindorfer D. ${ }^{1}$, Khoury J. ${ }^{2}$, Alwell K. ${ }^{1}$, Moomaw C. ', Woo D. ${ }^{1}$, Adeoye O. ${ }^{3}$, Flaherty M. ${ }^{1}$, Khatri P. ${ }^{1}$, Ferioli S. ${ }^{1}$, Broderick J. ${ }^{1}$, Kissela $B{ }^{1}$ \\ ${ }^{1}$ University of Cincinnati, Neurology, Cincinnati, USA, ${ }^{2}$ Cincinnati Children's Hospital, Cincinnati, USA, 3University of Cincinnati, Emergency Medicine, Cincinnati, USA}

Background: Transient ischemic attacks (TIAs) are followed by stroke within 30 days in $8-10 \%$ of cases. Some patients present with a history of multiple recent TIAs. Clinical teaching suggests that repeated, stereotyped transient events are less likely to progress to stroke. Therefore, we hypothesized that the risk of stroke for patients with multiple TIAs would be lower than for patients with a single TIA.

Methods: The Greater Cincinnati/Northern Kentucky Stroke Study ascertained all strokes and TIAs among its residents for 2005. TIA was defined as an event with focal neurologic deficits lasting $<24 \mathrm{hrs}$. The last event occurring during the study period was considered the index event.

Results: Among 3,379 subjects with $\geq 1$ stroke or TIA, the index event was an ischemic or hemorrhagic stroke for 2,501 (74\%) and a TIA for 878 (26\%); 100 strokes (4\%) and 178 TIAs (20\%) were preceded by a TIA within 90 days. Multiple TIAs, ranging from 2 to 34 events, occurred in 282 patients. The overall risk of stroke after TIA was $8.2 \%$ within 30 days and $10.2 \%$ within 90 days. For patients with a single TIA, risk of stroke was $9.3 \%$; for those with $\geq 2$ TIAs the risk was $13.6 \%(p=0.07)$.

Discussion: Our results, showing higher risk of stroke for patients with multiple TIAs, contradicts our original hypothesis. Thus, clinicians should remain vigilant for future stroke risk in TIA patients, even among patients with multiple similar TIAs in 
the recent past. Further analysis of time between events will be presented.

\section{SS8/6 \\ Validation of Emergency and Final Diagnosis Coding in Transient Ischaemic Attack (TIA)}

\author{
Ghia D. 1, Thomas P. ${ }^{2}$, Cordato D. ${ }^{3}$, Worthington J. ${ }^{3}$, \\ Hodgkinson S. ${ }^{3}$, Beran R. ${ }^{3}$ \\ ${ }^{1}$ Department of Neurophysiology, Liverpool Health \\ Service, Sydney, Australia, ${ }^{2}$ Department of Neurology, \\ Liverpool Health Service, Sydney, Australia, ${ }^{3}$ The \\ University of New South Wales, South West Sydney \\ Clinical School, Sydney, Australia
}

Background: Validity of hospital coding has important implications for stroke research using administrative data sets. Our aim was to determine accuracy of Emergency Department (ED) transient ischaemic attack diagnoses and the validity of hospital final diagnosis coding for TIA, stroke and mimic disorders in an Australian tertiary referral hospital.

Methods: Using administrative datasets we identified all patients with an ED diagnosis of TIA (435.9 ICD-9) admitted to Liverpool Hospital, Sydney Australia, from January 2003 to December 2007. ED and hospital admission records were matched and hospital final diagnosis (ICD-10-AM) codes recorded. All records were expertly reviewed to determine the validity of diagnostic coding.

Results: 570 patients were admitted with an ED diagnosis of TIA. According to hospital final diagnosis (ICD-10-AM) coding 263 patients $(46 \%)$ had TIA, $165(29 \%)$ a stroke and $142(25 \%)$ another (TIA mimic) diagnosis. Expert review determined a final diagnosis of TIA in 293 (51.4\%), stroke in 149 (26.1\%) and TIA mimic in $128(22.5 \%)$. Sensitivity and specificity of final hospital diagnoses (1CD-10-AM) were $79 \%$ and $88 \%$ for TIA, $86 \%$ and $91 \%$ for stroke and $87 \%$ and $93 \%$ for TIA mimic, respectively.

Conclusions: Only half of the emergency diagnoses retained a diagnosis of TIA. The 1CD-10-AM hospital final diagnosis codes for TIA, stroke and TIA mimic had high sensitivity and specificity at expert review.
SS9

Epilepsy and Other Seizure/ Neurological Disorders Epidemiology (Part 2)

August 29, 2009

15:00-16:00

\section{SS9/1 \\ Non-Epileptic Seizures: One Condition or Many?}

\section{Gigineishvili D., Shakarishvili $R$.}

Department of Neurology and Neurosurgery, Javakhishvili State University, Tbilisi, Georgia

Background: Recurrent paroxysmal events usually are regarded by physicians as epilepsy. If no positive evidence was found after investigation, the diagnosis of non-epileptic seizure is suspected. What and how many medical conditions may resemble epilepsy are still unclear, so the precise diagnostic categorization of seizure disorder would lead to appropriate management and better outcome.

Methods: Retrospective analysis of all patients' medical records hospitalized during one calendar year in the Sarajishvili Institute of Neurology \& Neurosurgery epilepsy unit. Multidisciplinary approach for determining nature of a paroxysmal disorder including special neuropsychiatric assessment.

Results: 136 patients $\geq 10$ years of age received clear discharge diagnosis. Of the 33 patients with established non-epileptic events, psychogenic origin was recognized in 26 cases (79\%). Other patients were diagnosed as having physiologic non-epileptic seizures. Among the latter, neurocardiogenic syncope was a predominant diagnosis (3 out 7), endocrine and metabolic disorders were seen in two cases. Somatoform and dissociative disorders were the most frequent current diagnoses in the psychogenic group (69\%). Remaining diagnoses included anxiety, stress related and personality disorder. Coexistence of epilepsy (active or in remission) and non-epileptic seizures was seen in four cases.

Conclusion: The results showed that epilepsy, psychiatric and cardiologic pathology are the conditions most frequently seen in the epilepsy unit. Therefore, physician should always consider mental and organic disorders other than epilepsy in the differential diagnosis of seizure disorder. Multidisciplinary diagnostic approach will help him in making an accurate diagnosis of nonepileptic events. 


\section{SS9/2 \\ Risk Factors for a First Epileptic Seizure Symptomatic of Brain Tumor: A Case Control Study}

\begin{abstract}
Leone M.A. ${ }^{1}$, Ivashynka A. ${ }^{2}$, Tonini C. ${ }^{3}$, Beghi E. ${ }^{4}$, ARES (Alcohol RElated Seizures) Group

${ }^{1}$ Clinica Neurologica, Ospedale Maggiore della Carità, Novara, Italy, ${ }^{2}$ National Neurology and Neurosurgery Research Center, Minsk, Belarus, ${ }^{3}$ Ospedale G. Salvini, Garbagnate M.ese, Italy, ${ }^{4}$ Istituto di Ricerche

Farmacologiche Mario Negri, Laboratorio di Malattie Neurologiche, Milano, Italy
\end{abstract}

Background: The risk of seizures is increased in patients with brain tumors, but not all risk factors are known. We aimed to identify factors that increase the risk of a first seizure in patients with brain tumor.

Methods: Multicenter case-control study of 102 patients with a first seizure symptomatic of brain tumor matched to 123 hospital stroke controls by center, gender and age.

Results: The risk of first seizure was evaluated for family history of seizures, pre- and peri-natal factors (complications of pregnancy, low gestational age, low birth weight, complications of delivery), post-natal factors (psychomotor retardation, febrile seizures, history of hypertension, metabolic alterations at admission, use of epileptogenic drugs, history of cerebral palsy), lifestyle factors (history of drug addiction, average daily intake of absolute alcohol). None of this significantly increased the risk of a first seizures, except a family history of seizures: Odds Ratio $(\mathrm{OR})=2.6,95 \%$ Confidence Limits $(\mathrm{CL})=1.0-7.8$. Regarding CT-scan characteristics, the risk increased for supratentorial lesions (9.1, 2.1-40.2) and for cortical involvement (4.6, 2.5-8.7), but not for size of the tumor, the presence of edema, cortical atrophy and leukoaraiosis. The risk was higher for astrocytoma and oligodendroglioma $(3.6 ; 1.3-11.2)$ than for metastasis $(0.9 ; 0.6$ 1.7), glioblastoma (0.4; 0.2-0.7), and meningioma $1.0(0.6-2.1)$.

Conclusions: Cortical involvement, supratentorial lesions, and a positive family history for seizures increase the risk of a first epileptic seizure in patients with brain tumors.

\section{SS9/3 \\ Current Difficulties of Epidemiological Studies in the UK: The Case of a Prospective Study of the Outcome of Epilepsy}

\section{Neligan A., Bell G.S., Shorvon S.D., Sander J.W. \\ Department of Clinical and Experimental Epilepsy, UCL Institute of Neurology, London, United Kingdom}

Introduction: The National General Practice Study of Epilepsy \& Epileptic seizures (NGPSE) is one of the largest prospective community based study of an incident cohort of patients with epilepsy. It was set up in 1984 to determine the long term prognosis for people with epileptic and febrile seizures. Due to changes in legislation no o follow-up data (other than mortality) has been obtained since 2001 .

Methods: The legal and administrative obstacles faced attempting to resume the study are described.

Results: After 20 months of applications to various ethical and administrative bodies at national and local level, we are still not able to proceed with the next stage of this study: namely to send questionnaires to the patients' General Practitioners to obtain further information regarding seizure status.

The study has been severely hindered by the dilution of the information we are allowed to obtain, differing legislation between the different constituent parts of the UK, and poor communication between the different administrative bodies.

Conclusions: Based on our experience, the legislative constraints introduced in the past 10 years have had a profound effect on epidemiology in the UK; whether such projects are now feasible remains in doubt. This impact is evidenced by the dwindling number of long-term epidemiological projects in existence and the proportion of research funding that such studies obtain.

It raises the sombre thought that UK-based epidemiology may not have a viable future.

\section{LB-SS/SS9/4 \\ Assessment of Response to Rehabilitative Treatments in Children and Adolescents: Improving the Inter-Rater Agreement}

\author{
Chiappedi M. ${ }^{1}$, Beghi E. ${ }^{2}$, Biserni P. ${ }^{3}$, Ferrari-Ginevra O. ${ }^{4}$, \\ Ghezzo A. ${ }^{5}$, Maggioni E. ${ }^{6}$, Mattana F. ${ }^{2}$, Spelta P. \\ Stefanini M.C. ${ }^{8}$, Tonali P.A. ${ }^{8}$ \\ ${ }^{1}$ Fondazione Don Carlo Gnocchi ONLUS, Salice Terme, \\ Italy, ${ }^{2}$ Mario Negri Institute, Milan, Italy, ${ }^{3}$ Don Carlo \\ Gnocchi ONLUS Foundation, Salice Terme, Italy, ${ }^{4}$ Don \\ Carlo Gnocchi ONLUS Foundation, Pessano con Bornago, \\ Italy, 5 Don Carlo Gnocchi ONLUS Foundation, Falconara, \\ Italy, ${ }^{6}$ Don Carlo Gnocchi ONLUS Foundation, Milano, Italy, \\ ${ }^{7}$ Don Carlo Gnocchi ONLUS Foundation, Inverigo, Italy, \\ ${ }^{8}$ Don Carlo Gnocchi ONLUS Foundation, Rome, Italy
}

Background: The response to cognitive and motor rehabilitation depends on subjective assessment and may vary across physicians. We wanted to test the inter-rater agreement (before and after training) in the evaluation of children's and adolescents' response to rehabilitative treatments.

Methods: Five child neurologists ('raters') were invited to draw 2-3 short case reports among

those most commonly seen. 14 case histories were presented and raters used a structured questionnaire to report changes after selected rehabilitation programs. Response was coded as 'Yes', 'No', or 'Uncertain' in different functional domains (Motor, Social, Alimentary, Communication, Personal Autonomy). Interrater agreement was measured using the kappa statistic and categorized as almost perfect $(>0.80)$, substantial $(0.61-0.80)$, moderate (0.41-0.60), fair (0.21-0.40), slight and poor $(<0.21)$. Raters where then asked to discuss any reason for disagreement. The test was repeated with different cases (16 case histories) adding a sixth rater, who had participated to the discussion. 
Results: The agreement mostly ranged from poor to good in the first test (worse for Social, Personal Autonomy and Communication). Training improved agreement in almost all domains. There were no frank outliers.

The agreement was lower with a specific approach (i.e. grouping 'Uncertain' to 'No') than with a sensitive approach (i.e. grouping 'Uncertain' to 'Yes').

Conclusions: Inter-rater agreement on the response to rehabilitation is variable but it improves after discussion; agreement in some functional domains (Social, Personal autonomy, Communication) is more difficult to obtain than in others.

\section{LB-SS/SS9/5}

\section{Predictors of Long Term Mortality in an Elderly Cohort. Preliminary Data from the NEDICES Cohort Study}

\author{
Morales J.M. ${ }^{1}$, Bermejo-Pareja F. ${ }^{2}$, Benito-León J.2, \\ Rodríguez C. ${ }^{2}$, Trincado R. ${ }^{3}$, Boix R. ${ }^{4}$, Vega $S .{ }^{5}$, \\ Medrano M.J. ${ }^{4}$, Neurological Disorders in Central Spain (N \\ ED/CES) Study Group \\ ${ }^{1}$ Ministry of Health and Social Policy, Department of \\ Research, Madrid, Spain, 'University Hospital '12 de \\ Octubre', and CIBER of Neurodegenerative Disorders, \\ Neurology, Madrid, Spain, ${ }^{3}$ University Hospital '12 de \\ Octubre', Neurology, Madrid, Spain, ${ }^{\prime}$ Institute Carlos III, \\ Epidemiology National Center, Madrid, Spain, ${ }^{5}$ Espinar \\ Health Center, El Espinar, Spain
}

Background: To evaluate the main predictors of mortality, detected on the baseline wave (1994-5), at ten year of followup, in the participants in the Neurological Disorders in Central Spain (NEDICES) Cohort Study, a population-based cohort study in the elderly with 5,278 screened participants. The mortality of this cohort has been evaluated by means of the National Death Registry of Spain (NDRS) until 31-12-2004.

Methods: At baseline, an evaluation of the demographic, life style and health status of all participants was performed. This evaluation measured subjective and objective (co-morbidity) health status and also the presence of several neurological disorders. Several statistical analyses (mainly Cox regression) to evaluate the main predictors of mortality in this cohort were performed.

Results: At ten years (mean follow-up time $=8.6 \pm 3.3$ years; total observation time $=45,338$ person-years), 1,810 participants were dead. Several demographics (age, sex, educational level) and life style (smoking and alcohol intake habits), subjective (in men) and objective health status data were predictors of mortality. Of the neurological disorders investigated, stroke in men $(\mathrm{HR}=1.49 \mathrm{CI} 95 \% 1.11-1.99)$, dementia in both men $(\mathrm{HR}=2.69$ CI95\% 1.96-3.70) and women $(\mathrm{HR}=2.61 \mathrm{CI} 95 \% 2.02-3.36)$ and Parkinson disease, men $(\mathrm{HR}=1.77 \mathrm{CI} 95 \% 1.13-2.77)$ and women $(\mathrm{HR}=2.05 \mathrm{CI} 95 \%$ 1.30-3.22) were predictors of mortality in Cox regression adjusted by age, and change of age by time and other co-morbidities.

Conclusions: Multiple factors contribute to increased risk of all cause mortality among older people. Stroke (in men), dementia and Parkinson disease at baseline were the main neurological predictors of mortality at ten years in this cohort.

\section{LB-SS/SS9/6 \\ Neurological Diseases at a Primary Care Outpatient Clinic in Mid-North Mato Grosso, Brazil in 2008}

\author{
Siqueira H. ${ }^{1}$, Dalbem J. ${ }^{1}$, Alvarenga $R^{2}{ }^{2}$ \\ 'UNIRIO, Neurology, Cuiaba, Brazil, ${ }^{2}$ UNIRIO, Neurology, \\ Rio de Janeiro, Brazil
}

The Family Health Program, based on a family doctor practice, was widely implemented using multidisciplinary teams that included specialists.

Objectives: The objective of this study was to describe the prevalence of neurological syndromes in mid-north Mato Grosso, Brazil, and to compare these findings with a study carried out in South America.

Methods: A prospective, descriptive study was carried out to analyze the demographic and clinical data of neurological patients who consulted in 2008 (new and follow-up cases). The 57 healthcare agents of the consortium who look after the 277,427 inhabitants of the region refer cases with neurological signs to this healthcare center.

Results: Of the 33,291 outpatient consultations carried out at this healthcare center, 1,402 consisted of neurological consultations, 633 adults and 769 children. The mean age of the adult patients was 38.9 years and the majority $(67.9 \%)$ was female. The most common syndrome were cephalea $(49.1 \%)$. Of the pediatric consultations, there was a slight predominance of boys $(62.5 \%)$ and the mean age was 8.5 years. The most common syndromes were attention deficit/hyperactivity disorder (ADHD) (27.4\%).

Discussion: The results found are in agreement with publications from other Latin American studies, describing the principal reasons for consultations with neurologists in primary care. With respect to the pediatric population, there was also agreement with respect to ADHD as being the most common reason for consultation.

Conclusion: Neuroepidemiological studies are fundamental for the elaboration of health policies, as well as for the elaboration of educational programs designed to train regional healthcare professionals. 
SS10

Parkinson's Disease and Other Movement Disorders - Epidemiology and Management (Part 2)

August 29, 2009

16:30 - 17:30

\section{SS10/1 \\ Parkinsonism in a Population of Northern Tanzania: A Community-Based Door-to-Door Study in Combination with a Prospective Hospital-Based Evaluation}

Winkler A. ${ }^{1}$, Tütüncü E. ${ }^{2}$, Trendafilova A. ${ }^{2}$, Meindl M. ${ }^{3}$, Kaaya J. ${ }^{4}$, Kassubek J. ${ }^{2}$, Schmutzhard E. ${ }^{3}$

${ }^{1}$ Departments of Interdisciplinary Palliative Care and Neurology, Ludwig-Maximilians-University, Munich, Germany, ${ }^{2}$ University of UIm, Neurology, Ulm, Germany, ${ }^{3}$ Medical University of Innsbruck, Neurology, Innsbruck, Austria, ${ }^{4}$ District General Hospital Babati, General Medicine, Babati, Tanzania, United Republic of Tanzania

Background: The prevalence of Parkinson's disease (PD) in sub-Saharan Africa is still a matter of debate. The few studies that have been conducted have shown prevalences lower than those in the western world. Whether this represents a genuine finding is unclear to date.

Methods: In northern Tanzania, we have conducted a hospital-based evaluation and a community-based, door-to-door study to assess the prevalence of Parkinsonism, including that of PD. Over a period of eight months, all patients admitted to a mid-size rural hospital were screened for PD. In parallel, 1569 people aged $\geq 50$ years were recruited from the communities and assessed for Parkinsonism with standard questions. Sampling was performed according to the method of 'multistage cluster sampling'. Questions were pre-tested in a pilot study prior to the survey. People who screened positive were examined by a specialist neurologist.

Results: In hospital, eight of 740 people with neurological diagnoses had Parkinsonism, of whom three patients had a diagnosis of PD. In the community-based study, eighteen people answered positively to least one of the 12 screening questions. However, after examination the diagnosis of PD could not be confirmed in any of them.

Conclusion: The prevalence of PD in northern Tanzania was found to be very low. This result would need confirmation in studies with larger populations, ideally of different African ethnicities.

\section{SS $10 / 2$}

\section{Essential Tremor Is Not Associated with a Long Term Increase in Mortality. Data from the NEDICES Cohort Study}

\author{
Bermejo-Pareja F.1, Trincado R. ${ }^{2}$, Benito-León J. ${ }^{1}$, \\ Villarejo A. ${ }^{2}$, Posada I.J. ${ }^{2}$, Rodríguez C. ${ }^{1}$, Medrano M.J. ${ }^{3}$, \\ Louis E.D. ${ }^{4}$, Neurological Disorders in Central Spain \\ (NED/CES) Study Group \\ 'University Hospital '12 de Octubre', CIBERNED, \\ Neurology, Madrid, Spain, University Hospital '12 de \\ Octubre', Neurology, Madrid, Spain, '3nstitute Carlos III, \\ Epidemiology National Center, Madrid, Spain, ${ }^{4} \mathrm{GH}$ \\ Sergievsky Center, Neurology, New York, USA
}

Background: To evaluate the long term mortality (5 and 10 years) after the baseline wave (1994) of essential tremor participants in the Neurological Disorders in Central Spain (NEDICES) Cohort Study, a population-based cohort study in the elderly (65 years and more) with 5,278 screened participants at baseline. Their mortality at 3 years was increased (Neurology, 2007; 69:1982-9). The mortality of this cohort has been evaluated by means of the National Death Registry of Spain from 1994 to 2004. Methods: At baseline, diagnosis of essential tremor was performed by neurologists. The mortality risk was analysed with Cox proportionalhazards regression model with time-dependent variables after making adjustments for potential covariates (age, sex, educational level and an index of co-morbidity). Survival study was performed with Kaplan-Meier analysis.

Results: The mortality at ten years in the 5,025 participants without essential tremor was $33.9 \%$ (1,705 persons). In 225 essential tremor participants it was $36.4 \%$ ( 82 persons). When adjusted for socio-demographic variables and co-morbidity in the Cox proportional hazards model, suffering from essential tremor was not associated with an increased risk of mortality (Hazard Ratio $(H R)=0.87$; CI 95\%: 0.69-1.09). Using the Kaplan-Meier method, mean survival was 8.63 years (CI95\%: 8.53-8.72) for participants without essential tremor and 8.60 years (CI95\%: 8.16-9.04) for essential tremor participants, log rank test for equality of survival $\mathrm{p}=0.515$. The results at five years were similar.

Conclusions: Long term mortality (five and ten years after baseline wave) in essential tremor participants is not increase in this cohort. 


\section{SS10/3 \\ Double-Blind Controlled Randomized Study of the Use of Levetiracetam to Treat Tics in Children and Adolescents with Tourette Syndrome}

Awaad Y

King Fahad Medical City, Pediatric Neurology, Riyadh, Saudi Arabia

Background: Some drugs currently used to treat tics have drawbacks, including the risk of side effects such as tardive dyskinesia. Therapeutic options with better safety profiles are needed. Levetiracetam is an antiepileptie drug with atypical GABAcrgic effects that might be beneficial for this indication. To evaluate the effects of Ievetiracetam on motor and vocal tics, behavior and school performance in children and adolescents with tics and Tourette syndrome.

24 patients, age 6-18 years with tics and Tourette syndrome were enrolled in this prospective, double- blinded placebo randomized study for 8 weeks. Each group had 12 patients. The initial starting dose of levetiracetam was $250 \mathrm{mg} / \mathrm{d}$. The dosage was titrated over 3 "'weeks to 1,000 to $2,000 \mathrm{mg} / \mathrm{d}$. Clinical out-comes were assessed with the Clinical Global Impression Scale, Yale Global Tie Severity Scale, and Revised Conners' Scale.

Results: 10 out of 12 patients in the Levetiracetam group showed improvements based on all of the scales used and 4 patients improved with regard to behavior and school performance. 2 patients dropped out. 9 patients out of 12 patients in the placebo group showed no improvement, one patient showed a great placebo effect, and 2 patients dropped out of the study. Levetiracetam was generally well tolerated. 2 patients discontinued because of exaggeration of pre-existing behavioral problems.

Conclusions: Levetiracetam may be useful in treating tics in children and adolescents. Given its established safety profile, levetiracctam is a candidate for additional evaluation.

SS10/4

\section{Determinants of Health-Related Quality of Life in Patients with Primary Focal Dystonia}

Pekmezovic T. ${ }^{1}$, Svetel M. ${ }^{2}$, Ivanovic N. ${ }^{2}$, Petrovic I. ${ }^{2}$, Kisic Tepavcevic D. ${ }^{3}$, Kostic V. ${ }^{2}$

${ }^{1}$ Institute of Epidemiology and Institute of Neurology,

Belgrade, Serbia, ${ }^{2}$ Institute of Neurology, Belgrade, Serbia, ${ }^{3}$ Institute of Epidemiology, Belgrade, Serbia

Background: Little is known about factors associated with a poor health-related quality of life (HR-QoL) in patients with primary focal dystonia. The aim of the study was to identify factors influencing HR-QoL among patients with focal dystonia.

Methods: The study comprised 157 consecutive patients with primary focal dystonia treated and followed at the Institute of Neurology, Belgrade. Our outcome measure for HR-QoL was SF36. In order to measure disease related factors, the Global Severity Scale (GBS) was used. The level of social participation was measured by the Leisure Activity Scale (LAS). Coping behavior was assessed by the Acceptance of Illness Scale (AIS) and the Stigma Scale (SS). The Multidimensional Personal Support Scale (MPSS) was used to measure quantity and quality of support from partners, friends, and family members. Self esteem and a tendency to self deprecate were measured by the Self Esteem Subscale (SES) and Self Deprecation Subscale (SDS).

Results: Male patients $(p=0.026)$ and patients who finished at least high school $(\mathrm{p}=0.001)$ had significantly higher levels of LAS. Married patients had significantly higher degree of MPSS $(\mathrm{p}=0.001)$. Patients with pain in region affected by dystonia had significantly higher score of GSS $(\mathrm{p}=0.001)$. Patients with higher GSS, AIS, SS, and SDS values, had significantly $(\mathrm{p}<0.01)$ lower scores of PF, RP, RE and MH of SF-36. Significant $(p<0.01)$ positive correlation was obtained between the LAS and PF, RP, and RE.

Conclusions: We concluded that the dystonic patients present with a wide range of social disabilities and impairments of HR-QoL.

\section{SS10/5 \\ Gene-Demography Analysis Unmasks Susceptibility of Never Smokers to Park 2 Related Parkinsonism}

Vinish M., Prabhakar S., Khullar M., Anand A.

Postgraduate Institute of Medical Education and

Research, Neurology, Chandigarh, India

Background: Parkinson's disease (PD) is a complex neurological disorder without any known genotype-demography associations among sporadic variants. In order to examine the contribution of mutations in PD patients and their relation to different variables, we screened 120,000 patients that visited Neurology clinic from 2001 to 2006 and studied the association of PARK 2 mutations with such variables in 70 of these patients.

Methods: We screened 70 PD samples by PCR-SSCP and sequencing. Demographic- religion data from randomly selected 1010 of 120,000 patients visiting Neurology clinic was compared with state data bases.

Results: PARK 2 mutations were observed among $40 \%$ of patients all of whom were never smokers. Sikhs from rural background exhibited majority of these mutations. The frequency of PARK 2 mutations among females was significantly high as compared to males (p-0.015). The age of onset of patients with rural background is significantly less as compared to patients with urban background $(\mathrm{p}=0.004)$. The socio economic and demographic spectrum of the outpatient and the background population was found to be comparable.

Conclusions: As PD patients with PARK 2 mutations were found to be sporadic variants and never smokers, a non-redundant inverse relationship between founder PARK 2 mutations and smoking is implicated for its unexpected high frequency. The predisposition of Sikhs to PARK 2 mutations necessitates larger study among its familial variants and smoker PD population given that religion matched never smoker controls did not display any mutations. 


\section{SS10/6 \\ Smoking, Apolipoprotein E Epsilon2 Allele and Parkinson's Disease Risk in Israel}

\author{
Peretz C. ${ }^{1}$, Bar-Shira A. ${ }^{2}$, Orr-Urtreger A. ${ }^{2}$, Giladi N. ${ }^{3}$ \\ ${ }^{1}$ Tel-Aviv University, Epidemiology, Tel Aviv, Israel, ${ }^{2}$ Tel Aviv \\ Sourasky Medical Center, Genetics, Tel Aviv, Israel, ${ }^{3}$ Tel \\ Aviv Sourasky Medical Center, Neurology, Tel Aviv, Israel
}

Background: Parkinson's disease (PD) is currently considered as a multi-factorial disease caused by the interaction between genetic and environmental factors. Many epidemiological studies have shown a negative association between PD and smoking. The role of Apolipoprotein-E (APOE) polymorphism as a risk marker for PD is not clear. We aimed to investigate the relationship between smoking (ever/never), APOE and the risk of PD.

Methods: We designed a case-controls (1:2) study, with 150 non-demented, PD patients (cases) age 50-75, 150 spouses (first control group) and 150 patients with osteoarthritis (OA) (second control group). APOE genotype was assessed from either blood or saliva.

Results: The present analysis is based on $121 \mathrm{PD}$ patients, 84 PD patients' spouses and 106 patients with OA.

Ever smoking and carrying APOE-E2 were found to be less frequent among the PD patients when compared to the 2 controlgroups, but these differences were not significant. For smoking the ORs were $0.66,0.70(\mathrm{p}=.13, \mathrm{p}=.19)$ and for APOE-E2 the ORs were $0.49,0.72(\mathrm{p}=.06, \mathrm{p}=.44)$. When the interaction of these two factors- smoking*APOE-E2 was tested, we found a significant interaction effect when the PDs were compared to the 2 control-groups $(\mathrm{p}=.00, \mathrm{p}=.01)$. Only among smokers, carrying the APOE-E2 reduced significantly the risk of PD as follows: $\mathrm{OR}=0.24(\mathrm{p}=.0347)$ in the comparison of the PDs to the spouses, and $\mathrm{OR}=0.15(\mathrm{p}=.0023)$ in the comparison of the PDs to the OAs.

Conclusions: Carrying the APOE-E2 haplotype might have a protective effect for PD among ever smokers but this should be further investigated.
SS11

Stroke Epidemiology and Management (Part 4)

August 29, 2009

16:30-17:30

\section{SS11/1 \\ Stroke Recurrence in Akita, Japan \\ Suzuki K. ${ }^{1}$, Nagata K. ${ }^{2}$, Izumi M. ${ }^{3}$ \\ ${ }^{1}$ Research Institute for Brain and Vessels, Epidemiology, Akita, Japan, ${ }^{2}$ Research Institute for Brain and Blood Vessels, Neurology, Akita, Japan, ${ }^{3}$ Research Institute for Brain and Vessels, Cardiology, Akita, Japan}

Background: Although the recurrent stroke gives further physical disability and mental deterioration to the stroke victims, there are a few systematic epidemiological studies concerning stroke recurrence. Akita stroke onset registry (ASOR) which started in 1973 is the biggest epidemiological stroke registry in Japan. We analyzed the recurrent stroke based on the ASOR data.

Methods: We have traced 55,003 first-ever stroke cases in whom the diagnosis of stroke subtype was confirmed on the CT and/or MRI between 1983 and 2004. The diagnosis of the recurrent stroke was also confirmed on CT and/or MRI upon admission.

Results: 6,420 of $55,003(12 \%)$ cases had recurrent stroke: 5,463 cases had 2 stroke events, 812 cases had 3 events, 145 cases had 4 events, 33 cases had 5 events, and 5 cases had 6 events. One case had 7 events and another case had 8 events. In the analysis of stroke subtypes, $23 \%$ of those with cerebral infarction (CI) had recurrent stroke, $18 \%$ of those with cerebral hemorrhage $(\mathrm{CH})$ had recurrence, and $7 \%$ of those with subarachnoid hemorrhage (SAH) had recurrence. The stroke subtypes of recurrent stroke seem more complicated. In the recurrence of CI cases, $84 \%$ had $\mathrm{CI}, 14 \%$ had $\mathrm{CH}$ and $2 \%$ had SAH. In the recurrence of $\mathrm{CH}$ cases, $56 \%$ had $\mathrm{CH}$, and 41 had CI. Only $23 \%$ of those with SAH had $\mathrm{SAH}$ in the recurrence.

Conclusion: The complex configuration of subtypes in recurrent stroke may be linked to the diversity in the clinical strategy of prevention of recurrence. 


\section{SS11/2}

\section{Relationship Between ABCD ${ }^{2}$ Score and Early Risk of Stroke After a Transient Ischemic Attack in Chinese People}

\author{
Tan S., Xu Y., Zhao L., Song B., Li Z., Zhang R., Li S., Gao Y. \\ The First Affiliated Hospital of Zhengzhou University, \\ Neurology Department, Zhengzhou, China
}

Background: It was reported that the $\mathrm{ABCD}^{2}$ Score predicted well the early stroke after Transient Ischemic Attack (TIA). However, the similar studies aren't still found in yellow people. The study is to investigate the relationship between $\mathrm{ABCD}^{2}$ Score and stroke risk after TIA in Chinese people.

Methods: 1. Between 2007/02/01 and 2008/03/01, Consecutive inpatients and outpatients with TIA within first $48 \mathrm{~h}$ of symptom onset were prospectively registered and scored by $\mathrm{ABCD}^{2}$ in neurology department of the First Affiliated Hospital of Zhengzhou University. The follow-up was performed at day 2 and day 7 after TIA. 2. The log-rank test was utilized to analyze the differences in stroke-free survivals between groups stratified by $\mathrm{ABCD}^{2}$ score. Logistic regression was used to determine independent prediction of $\mathrm{ABCD}^{2}$ score for subsequent stroke.

Results: 136 patients with TIA were included. Subsequent stroke was observed in 9 patients at day 2 and in 16 patients at day 7 after TIA. The risk of stroke increased according to the increase of the $\mathrm{ABCD}^{2}$ score (Table 1). Multivariate analysis showed that $\mathrm{ABCD}^{2}$ score $\geq 4$ independently associated with subsequent stroke at 2-day(OR9.578, 95\%CI 1.146-80.059, $\mathrm{P}=0.037)$ and at 7-day(OR13.458, 95\%CI 2.516-71.978, $\mathrm{P}=0.002$ ). ABCD ${ }^{2}$ score $\geq 4$ had a positive predictive value of stroke of $88.9 \%$ at 2-day and $87.5 \%$ at 7 -day.

Conclusions: High $\mathrm{ABCD}^{2}$ score in patients with TIA independently associated with subsequent stroke in Henan Chinese population. $\mathrm{ABCD}^{2}$ score $\geq 4$ has potential value as an indicator of high risk of very early stroke after TIA. Further studies in larger sample are needed.

\section{SS11/3 \\ Mortality Due to Stroke in Uruguay}

Hackembruch H., Perna A., Ketzoian C.N.

Institute of Neurology, Neuroepidemiology Section, Montevideo, Uruguay

Objectives: To determine mortality rate due to stroke in Uruguay over the last 50 years, to compare it with international figures and to study modifications in the trends of mortality.

Methods: The study was retrospective.

Global as well as age and sex standardized rates due to stroke were calculated for Uruguay and for other countries.

Changes in trends of death for stroke taking into account new diagnostic techniques and new treatments were studied.

The linear regression analysis method was used to establish the trend of mortality throughout these years.
Results: Mortality rate showed an increasing trend from 1950 to $1971\left(\mathrm{r}^{2}=0.859, \mathrm{p}=0.0001\right)$. The maximum peak was recorded in 1971. From 1971 to 2004, figures decreased $\left(r^{2}=0.949\right.$, $\mathrm{p}=0.0001$ ) reaching in 2004113 cases per 100,000 inhabitants. This inflexion point coincided with the introduction of intensive care units (ICU) as from 1970. Computed tomography (CT scan) was introduced in 1980 .

When comparing Uruguay to other countries of the region and the world, we find that Uruguayan mortality rate is similar to the one in Brazil, higher than in other American countries and below East European countries.

Discussion: The results of this study indicate that mortality rates in Uruguay are at an intermediate level on an international scale; as far as the region is concerned, Uruguay is at a disadvantage.

Prevention campaigns and the establishment of specialized units for the treatment of stroke are the way to continue with this decreasing trend.

\section{SS11/4 \\ Regional Differences in Stroke Mortality Rates in Chile: Analysis of Socioeconomic and Cardiovascular Variables}

\author{
Diaz V. ${ }^{1}$, Lavados P. ${ }^{2}$, Delgado I. ${ }^{3}$, Jadue L. ${ }^{3}$ \\ ${ }^{1}$ Universidad de Chile, Neurology and Neurosurgery, \\ Santiago de Chile, Chile, ${ }^{2}$ Clinica Alemana de Santiago, \\ Department of Medicine, Santiago de Chile, Chile, \\ ${ }^{3}$ Universidad del Desarrollo, Epidemiology and Public \\ Health Department, Santiago de Chile, Chile
}

There are same reports showing regional differences in Stroke Mortality Rates (SMR). The cause is unclear.

Aims: To analyze regional SMR and to investigate the contributions of socioeconomic and cardiovascular variables.

Methods: Ecological study. The demographic variables were obtained from death certificates of categories I 60 to I 69 and $\mathrm{G}$ 45 and G 46 (CI-10). Education, income, poverty, rural or urban residence were obtained from national socioeconomic characterization (CASEN). The cardio vascular variables: were obtained from Quality of Live Questionnaire (MINSAL). We calculated age, sex, rural and urban SMR. Using lineal regression models, logistic regression models, and lineal multivariate models we investigated the correlation of SMR with independent variables.

Results: 83.672 deaths and $7.975(9.5 \%)$ were strokes. We found significant differences in regional SMR adjusted by sex and age. Bio-Bio had the biggest rate of stroke, 1.6 time of the national mean (50.1 x 100.000) and Aysén smallest one, 0.4. Santiago had a rate minor to the national mean (43.6 $\mathrm{x} 100.000)$. Age and poverty of the region were the selected variables that explained the standardized mortality ratio. The lineal model for regional differences selected cardiovascular risk of Framingham, sedentary life, diabetes, and hypertension, but they explain only $52 \%$ of the stroke mortality variability. A national lineal model with all vriables, socioeconomic and cardiovascular risk factors was even smaller $\left(r^{2}\right.$ of $\left.18 \%\right)$. 
Conclusions: The contribution of socioeconomic and cardiovascular variables is limited; probably there are other factors, like quality of medical services that might have a greater impact.

SS11/5

\section{A Randomized Clinical Trial in Comparison Between the Two Kinds of Operations in Treating Intracerebral Hemorrhage}

Wang W.Z. ${ }^{1}$, Liu H.M. ${ }^{2}$, Li D. ${ }^{2}$

${ }^{1}$ Beijing Neurosurgical Institute, Neuroepidemiology, Beijing, China, ${ }^{2}$ Beijing Neurosurgical Institute, Beijing, China

Objective: To assess and compare the efficacy between the microinvasive craniopuncture therapy and the clearance of hematoma by craniotomy with small bone flap in treating patients with $40 \sim 80 \mathrm{ml}$ hemorrhage in the basal ganglion of the brain.

Methods: A multi-center, randomized case-control method was used in this clinical trial. 304 cases of intracerebral hemorrhage in the basal ganglion of the brain from 22 hospitals were selected randomly to be entered the study. Using the method of telephone, the center for quality control told a randomized number to every calling hospital, which divided the patient into one of the two groups randomly (one was microinvasive craniopuncture group, and the other was craniotomy with small bone flap group).

Results: 159 cases were entered in the microinvasive craniopuncture group and 145 cases in the clearance of hematoma by craniotomy with small bone flap group. This study revealed that the main results as follows: (1) By the end of the $3^{\text {rd }}$ month, there was a significant difference in patients with favorable outcomes (Barthel index score $\geq 95)$ between the two groups $\left(X^{2}=3.95, \mathrm{P}<\right.$ $0.05)$, which showed a better prognosis in microinvasive craniopuncture group. (2) There was a remarkable decrease in cases fatality by the end of the $3^{\text {rd }}$ month in the microinvasive craniopuncture group, which showed a statistically significant between the two groups $\left(X^{2}=5.35, \mathrm{p}=0.02\right)$.

Conclusions: Compared with the control group, the microinvasive craniopuncture therapy was verified to reduce the case fatality by the end of the $3^{\text {rd }}$ month.

\section{SS11/6 \\ Predicting 30-Day Mortality in Sub-Saharan Black Africans with Spontaneous Parenchymal Brain Hemorrhage: Data from a Tertiary Hospital in Cameroon}

Lekoubou Looti A Z ${ }^{1}$ Njamnshi K.A. ${ }^{2}$ Djientcheu VD.P. Jemea B. ${ }^{4}$, Essono Mba R. ${ }^{4}$, Ndoumbe A. ${ }^{5}$, Nkam M. ${ }^{6}$

${ }^{1}$ Faculty of Medicine and Biomedical Sciences, University of Yaounde I, Neurology Service Central Hospital Yaounde, Intensive Care Unit YUHC, Internal Medicine, Yaounde, Cameroon, ${ }^{2}$ Faculty of Medicine and Biomedical Sciences, University of Yaounde I, Neurology Service Yaounde Central Hospital, Internal Medicine, Yaounde, Cameroon, ${ }^{3}$ Faculty of Medicine and Biomedical Sciences, University of Yaounde I, Neurosurgery Service Central Hospital Yaounde, Surgery and Specialties, Yaounde, Cameroon, ${ }^{4}$ Yaounde University Hospital Centre, Intensive Care Unit, Yaounde, Cameroon, ${ }^{5}$ Faculty of Medicine and Biomedical Sciences, University of Yaounde I, Neurosurgery Service YUHC, Surgery and Specialties, Yaounde, Cameroon, ${ }^{6}$ Faculty of Medicine and Biomedical Sciences, University of Yaounde I, Yaounde University Hospital Centre, Intensive Care Unit, Yaounde, Cameroon

Background: Despite a high proportion of hemorrhagic stroke in SSA countries, available early outcome predictive models after spontaneous parenchymal brain hemorrhage (SPBH) have been developed essentially out of the African continent. This study was designed to identify independent admission predictors of 30-day mortality after SPBH.

Methods: Admission records of all patients with CT-scan confirmed SPBH seen at the Yaounde teaching hospital from January 2004 through December 2008 were reviewed. The following variables age, sex, Glasgow Coma Scale (GCS), pupillary abnormalities, mean arterial pressure, pulse pressure, temperature, the presence of an infection, location of hematoma, parenchymal hemorrhage volume (PHV) and the presence of Intra-Ventricular Hemorrhage (IVH) were analyzed. Independent predictors of 30-day mortality were identified using logistic regression.

Results: Overall, We reviewed 97 consecutive files. 30-daymortality rate was $50 \%$ without seasonal variations. Independent predictive admission factors of 30-day mortality were the Glasgow coma scale (GCS $\leq 8$ vs. GCS $>8, P=0.001)$, the volume of hematoma ( $\geq 30 \mathrm{~mL}$ vs. $<30 \mathrm{~mL}, \mathrm{P}=0.048)$ and the presence of intraventricular hemorrhage $(\mathrm{P}=0.022)$. Age and infratentorial location were not predictors.

Conclusion: Mortality in patients with SPBH is high in our setting. Mortality risk could be easily identified using 3 easy-toassess predictors of early mortality. 
SS12

Motor Neuron and Parkinson's Disease Epidemiology

August 29, 2009

16:30-17:30
SS12/2

\section{Incidence and Clinical Features of Amyotrophic Lateral Sclerosis (ALS) in More and Romsdal County, Norway}

Gundersen M.D., Yaseen R., Midgard R.

Department of Neurology, Molde Hospital, Molde, Norway

Background: We studied the incidence of ALS from 1988 to 2007 in Møre and Romsdal County, Norway. There are few incidence studies of ALS in Norway. In Hordaland County, the ALS-incidence was $1,60 / 10^{5}$ in $1979-89$. The ALS-mortality rates in Norway have increased during the last decades. The mortality rate in Møre and Romsdal was 1,54/10 $0^{5}$ in 1961-69 increasing to 2.40 in 1990-94. This is the first ALS-incidence study performed in the county.

Methods: Retrospective study using the patient database at the Department of Neurology, Molde Hospital. Patient records from 1988 to 2007 were identified searching the classification codes (ICD-9, ICD-10) for motor neuron disease. The hospital serves a stable, homogenous population of 246,000. We recorded gender, age, municipality, symptoms and signs, time between onset, diagnosis and death.

Results: 92 patients met inclusion criteria. The mean crude annual incidence was $1.90 / 10^{5}$ in $1988-2007 ; 2.11$ in men and 1.70 in women. Mean age at onset was 66.3 in men and 70.8 in women. The mean interval from onset to diagnosis was 13.3 months, and the mean survival interval from diagnosis was 17.3 months. 66 patients $(71.8 \%)$ met the El Escorial \& Airlie House criteria at diagnosis.

Conclusion: The present study shows an annual ALSincidence in Møre and Romsdal County, Norway 1988-2007 of similar magnitude as previous studies from the Nordic countries during the past years. The short survival time corroborates prior studies and is stable throughout the two studied decades. one with a neurological (non-degener non-neurological (other than orthopedic) disease. Only traumatic events occurred before the onset of symptoms were recorded. For any trauma, the resulting functional disability (whether temporary or permanent) was reported Based on power calculations, the sample was to include 400 cases and 800 controls.

Results: The study is ongoing. The present sample includes 225 cases, 151 neurological controls and 162 non-neurological controls. One or more traumatic events were reported by 125 cases (55.6\%) and 154 controls (49.2\%)(OR 1.3; 95\% CI 0.9-1.8). One or more disabling events were reported by 79 cases $(35.1 \%)$ and 104 controls $(33.2 \%)$ (OR 1.1; 95\% 0.7-1.6). Compared to controls, patients with ALS had a slightly but non-significantly higher risk of reporting $3+$ traumatic events (OR 1.7; 95\% 0.83.6).

Conclusions: Antecedent trauma is not a risk factor for ALS.
SS12/3

The Use of Mortality Data As Surrogate of Incidence in Amyotrophic Lateral Sclerosis

Marin B. ${ }^{1}$, Couratier P. ${ }^{2}$, Preux P.M. ${ }^{1}$, Logroscino G. ${ }^{3}$

${ }^{1}$ Institut de NeuroEpidémiologie et de Neurologie Tropicale, EA 3174 NeuroEpidémiologie Tropicale et Comparée, Limoges, France, ${ }^{2} \mathrm{CHU}$ Limoges, Service de Neurologie, Centre Expert SLA, Limoges, France, ${ }^{3}$ Department of Neurology and Psychiatry, School of Medicine, University of Bari, Bari, Italy

Introduction: Assessing incidence rates for Amyotrophic Lateral Sclerosis (ALS) through morbidity studies is unusual due to uncertain feasibility and high costs. Mortality data have frequently been used to estimate incidence but the accuracy of such a perspective has to be questioned. Our aim, through a systematic review, was to identify the methodological prerequisite allowing the use of mortality rates of ALS as surrogate of incidence rates. 
Methods: A Medline literature search was performed to identify until 2009, studies on ALS and motor neuron disease (MND) mortality rates. The mesh-terms were 'ALS', 'MND', 'mortality', 'incidence'. Additional references were identified from article citations.

Results and Discussion: We propose 5 methodological points to be critically assessed when considering a mortality-databased study. (1) Mortality data must be based on " underlying " and « contributory » causes of death. (2) A study (that could be nested in mortality study) about the quality/accuracy of deaths certificates should be performed or available for the country and period of time that is investigated (true positivity rate, positive predictive value, proportion of unknown causes overall). (3) To compare mortality data among calendar times, using a uniform international classification of diseases (ICD) is essential (ICD 6-8 and 10 on one hand, and 9 on the other hand). (4) To compare mortality data among regions, comparable health care system and death certificate system (in term of high quality) is essential. (5) To compare mortality data between ethnic groups, critical approach of data (ethnic, access to health care) is indispensable.

\section{LB-SS/SS12/4 \\ Prognostic Significance of Various Nutritional Parameters in Patients Suffering from Amyotrophic Lateral Sclerosis}

\author{
Kajeu P.-J. ${ }^{1}$, Marin B. ${ }^{1}$, Desport J.C. ${ }^{2}$, Preux P.M. ${ }^{1}$, \\ Couratier $P^{3}$ \\ ${ }^{1}$ Université de Limoges, IFR 145 GEIST, Institut \\ d'Epidémiologie Neurologique et de Neurologie Tropicale, \\ EA 3174 NeuroEpidémiologie Tropicale et Comparée, \\ Limoges, France, ${ }^{2} \mathrm{CHU}$ Limoges, Service d'Hépato- \\ Gastro-Entérologie, Unité Fonctionnelle de Nutrition, \\ Limoges, France, ${ }^{3} \mathrm{CHU}$ Limoges, Service de Neurologie, \\ Limoges, France
}

Background: Malnutrition has been shown to be associated with survival in patients suffering from ALS. Malnutrition can be evaluated with weight loss, body mass index and other more sophisticated methods. Our aim was to assess the independently prognostic significance of various nutritional markers in survival of patients suffering from ALS.

Methods: Our study included all patients with available nutritional evaluation during their follow up in our referral center located in France. Evaluated variables were body mass index, weight, tricipital skin-fold thickness, midarm muscular circumference, and markers obtained thanks to total body impedance analysis : phase angle, lean mass, hydratation disorders (extracellular to intracellular water ratio $(\mathrm{E} / \mathrm{I}))$. We used the cox proportional hazard model to perform a survival analysis from time to diagnosis until death or censoring time. We considered variables collected at first evaluation as fixed variables and during the entire follow up as time varying covariates. Our analyses were adjusted on sociodemographic characteristics and neurological clinical status at baseline.

Results: Among 175 patients with at least one nutritional evaluation, we identified that an increase in phase angle was sig- nificantly and independently associated with a better survival and that hydratation disorders (extracellular to intracellular water ratio (E/I) increment) was significantly associated with a shorter survival.

Discussion: The identification of newly prognosis factors of ALS can provide useful informations for clinicians to monitor patients and for researchers to give birth hypotheses as regards physiopathology of the disease and target for the management of patients.

\section{LB-SS/SS12/5 \\ A Prospective Study of Glycemic Status and Risk of Parkinson's Disease (PD)}

Van Den Eeden S.K., Shan J., Albers K., Quesenberry C., Ferrara $A$.

Kaiser Permanente, Division of Research, Oakland, USA

Background: Diabetes has been found to increase the risk of PD in some but not all studies. We sought to estimate the risk of PD associated with blood glucose levels in a prospective cohort.

Methods: Participants were members of the Multiphasic Health Checkup (MHC) Cohort at Kaiser Permanente (KP) with data on socioeconomic, medical history, lifestyle and body habitus measures and random serum glucose and/or a 1-hour post-load (75 gram) glucose challenge tests obtained from 1964-1973. PD diagnoses $(n=1,303)$ were obtained and confirmed from electronic medical records from 1997-2008. End of follow was the earliest of a PD diagnosis, death, or leaving the KPNC system. The study cohort had a mean follow-up of approximately 36 years and a total of $1,802,492$ person-years contributed. Proportional hazard models were used to analyze these data.

Results: The risk of PD increased slightly with increasing levels of glucose. Relative to the lowest category, the $2^{\text {nd }}$ through $4^{\text {th }}$ (highest) quartile hazard ratios $(95 \% \mathrm{CI})$ were $1.06(0.88$ $1.27), 1.11$ (0.93-1.33), 1.14 (0.95-1.37), respectively. The hazard ratio for diabetes (relative to low glucose quartile) was 1.34 (0.921.94). We also assessed individuals who underwent a 1-hr glucose test. Relative to the $<140 \mathrm{mg} / \mathrm{dl}$, those in the $140-199 \mathrm{mg} / \mathrm{dl}$, $>200 \mathrm{mg} / \mathrm{dl}$, and diabetes (at baseline) categories had HRs of 1.12 (0.97-1.28), 1.12 (0.94-1.34) and 1.24 (0.85-1.80), respectively. Adjustment for BMI did not alter these results.

Conclusions: These results are consistent with another large prospective study that found having type 2 diabetes modestly increased the risk of PD. 
SS13

Stroke Epidemiology and Management (Part 5)

August 30, 2009

08:00-09:00

SS13/1

\section{Incidence of Spontaneous Intracerebral Hemorrhage by Localization in a Hispanic- Mestizo Population in Iquique, Chile: The PISCIS Study}

\author{
Lavados P. ${ }^{1}$, Sacks C. ${ }^{2}$, Prina L. ${ }^{3}$, Escobar A. ${ }^{3}$, Tossi C. ${ }^{3}$, \\ Salinas $R^{4}$ \\ ${ }^{1}$ Department of Medicine and Department of Neurological \\ Sciences, Clinica Alemana-Universidad del Desarrollo \& \\ Universidad de Chile, Neurology Service, Santiago, Chile, \\ 2Department of Medicine, Universidad de Valparaíso, \\ Cátedra de Neurología, Valparaíso, Chile, ${ }^{3}$ Department of \\ Medicine, Hospital Regional de lquique, Neurology Unit, \\ Iquique, Chile, ${ }^{4}$ Universidad de Chile, School of Public \\ Health, Santiago, Chile
}

Background: The incidence of intracerebral hemorrhage (ICH) seems be higher in Hispanic communities. Our aim was to prospectively ascertain the incidence of first-ever spontaneous intracerebral hemorrhage $(\mathrm{SICH})$ stratified by localization in a Hispanic-Mestizo population of the northern region of Chile.

Methods: Between July 2000 and June 2002 all possible cases of ICH were ascertained from multiple overlapping sources and were rapidly assessed by two field neurologists. All ascertained cases were adjudicated by 2 stroke neurologists according to localization and followed at six months. Cases with vascular malformations or non-adjudicable localizations were excluded from the analysis. Annual incidence rates were age-adjusted to reference populations in order to allow comparisons. Associations were analyzed with a regression model.

Results: A total of 69 cases of first-ever ICH were identified. After exclusion, 64 cases (92.7\%) were considered to have SICH of which 58 cases $(84 \%)$ were adjudicated to lobar or non-lobar localization. The age-adjusted incidences to the world population according to localization were: Non-lobar 13.8 and lobar 4.9 per 100,000. Older women had a higher risk of lobar SICH and young men of non-lobar SICH. Hypertension was the most frequent cardiovascular risk factor in both localizations. No significant association was found between localization and age, gender, hypertension, diabetes or antithrombotic use. Prognosis was similar in both localizations

Conclusions: The incidence and prognosis of SICH according to localization in this population was similar to that reported previously in other communities. Hypertension or other cardiovascular risk factors were not associated with a particular localization.

\section{SS13/2}

\section{Awareness of Risk Factors and Warning Signs Among Cameroonian Patients at High Risk of Stroke is Poor}

\author{
Lekoubou Looti A.Z. ', Djientcheu V.d.P.', \\ Nghemmogne Takam C. ${ }^{3}$, Njamnshi K. A. ${ }^{4}$ \\ ${ }^{1}$ Faculty of Medicine and Biomedical Sciences, University \\ of Yaounde I, Internal Medicine, Yaounde, Cameroon, \\ ${ }^{2}$ Service of Neurosurgery Central Hospital Yaounde, \\ Faculty of Medicine and Biomedical Sciences University of \\ Yaounde I, Yaounde, Cameroon, ㄴuniversité des \\ Montagnes, Faculty of health sciences, Bagangté, \\ Cameroon, ${ }^{4}$ Service of Neurology Central Hospital \\ Yaoundé, Faculty of Medicine and Biomedical Sciences \\ University of Yaounde I, Yaounde, Cameroon
}

Background: Despite the high morbidity and mortality of stroke in Cameroon, no information on awareness of stroke risk factor and warning signs is available in Cameroonians at risk.

Methods: A hospital-based, cross-sectional study conducted in two services of the largest teaching hospital of YaoundeCameroon. Patients with hypertension and/or diabetes were interviewed. The primary composite end-point was the knowledge of at least one stroke risk factor and at least one stroke warning sign.

Results: There were 243 responders out 291 interviewed with a mean age of $58 \pm 10$ years. Only $33 \%$ could identify at least one warning sign of stroke, mainly hemiplegia. In the final logistic regression model, the ability to identify at least one stroke risk factor was the main significant predictor of the knowledge of at least one stroke warning sign $(\beta=3,425,95 \%$ confidence interval $[\mathrm{CI}]=2.503-4.347, \mathrm{P}<0.001) .42 \%$ could correctly give at least one risk factor for stroke, most commonly diabetes $(33 \%)$ and hypertension $(32.5 \%)$. In multivariate logistic regression, a university or higher education level $(\beta=2.577, \mathrm{CI}=1.419-3.735$, $\mathrm{P}<0.001)$, a family history of stroke $(\beta=1,313, \mathrm{CI}=1.014$ $13.615, \mathrm{P}=0.048)$ and the ability to identify at least one warning sign of stroke $(\beta=3.502, \mathrm{CI}=2.533-4.470 \mathrm{P}<0.001)$ emerged as independent predictors of the knowledge of at least one stroke risk factor.

Conclusion: The level of awareness of stroke risk factors and warning signs is low among Cameroonian patients at increased risk for the disease. These results will serve as basis to improve education on stroke. 


\section{SS13/3}

\section{Is Transcranial Magnetic Stimulation a Useful Clinical Adjunct for Predicting Stroke Risk Following Transient Ischemic Attack? A Prospective Cohort Study}

\author{
Edwards J. ${ }^{1}$, Boyd L. ${ }^{2}$, Stinear C. ${ }^{3}$, Teal P. , Sobolev B. ${ }^{1}$, \\ Meehan S. ${ }^{5}$, Levy $A .{ }^{1}$ \\ ${ }^{1}$ University of British Columbia, School of Population and \\ Public Health, Vancouver, Canada, ${ }^{2}$ University of British \\ Columbia, Physical Therapy, Vancouver, Canada, \\ ${ }^{3}$ University of Auckland, Faculty of Medical and Health \\ Sciences, Auckland, New Zealand, ${ }^{4}$ University of British \\ Columbia, Neurology, Vancouver, Canada, ${ }^{5}$ University of \\ British Columbia, Rehabilitation Sciences, Vancouver, \\ Canada
}

Background: Transient ischemic attack (TIA) is an important predictor of stroke and stroke risk after TIA is considerable. However, efforts to estimate this risk have yielded inconsistent results. The purpose of this study is to determine if cortical excitability, measured using Transcranial Magnetic Stimulation (TMS), predicts stroke following motor TIA. We propose that TIA alters intracortical excitability, measured as a raised threshold for intracortical inhibition in the affected vs. unaffected primary motor cortex (M1) during TMS. Further, we hypothesize that altered excitability predicts an increased risk of stroke after TIA.

Methods: In this prospective cohort study, the exposure is interhemispheric asymmetry in intracortical thresholds for inhibition and facilitation during paired-pulse TMS in individuals with acute motor TIA. The primary outcome measure is stroke within 90 days of TIA. We calculated asymmetry indices for intracortical inhibition and facilitation thresholds after TIA and, using Cox regression, will evaluate the relationship between these indices and 90-day stroke risk.

Results: Results showed significant asymmetry in intracortical thresholds following TIA. We observed an increased threshold for inhibition in the affected vs. unaffected M1 and slightly higher thresholds for facilitation. No secondary events at 90 days have yet been reported.

Conclusions: This study will advance the understanding of TIA neurophysiology and determine the clinical utility of TMS in estimating secondary stroke risk. Data showed higher inhibitory thresholds in the TIA-affected M1, suggesting that interhemispheric asymmetry persists after clinical symptoms have resolved. Results will also delineate the relationship between excitability thresholds following TIA and 90-day stroke occurrence.

\section{SS13/4 \\ Seasonal and Meteorological Variations in Cerebral Stroke $(\mathrm{CI})$ Incidence in Central European Country}

\author{
Bartko D. ${ }^{1}$, Combor I. ${ }^{2}$, Blazicek P. ${ }^{3}$, Madarasz S. ${ }^{3}$, \\ Kovacik M. ${ }^{3}$, Petrik O. ${ }^{3}$, Danisova J. ${ }^{4}$ \\ ${ }^{1}$ Central Military University Hospital, Institute of Medical \\ Sciences, Neurosciences, Military Health, Ruzomberok, \\ Slovakia, ${ }^{2}$ Central Military University Hospital, \\ Ruzomberok, Slovakia, ${ }^{3}$ Department of Neurology, Central \\ Military University Hospital, Ruzomberok, Slovakia, \\ ${ }^{4}$ Department of Neurology, University Hospital, Bratislava, \\ Slovakia
}

Background: Seasonal and meteorological variation on CI onset has been reported in various areas. Controversies/discrepancies exist.

Aim: 1. Seasonal variation in stroke incidence (region Bratislava-Slovakia).

2. One year stroke registry data,

3. Relationship between actual values and inter/intradiurnal changes of atmospheric pressure (AtP), temperature (AtT), humidity (atmospheric steam pressure (AtSp), equivalent temperature (eT) and CI incidence.

Material: 1632 pts with clear dg of CI. Seasonal/meteorological data, stroke registry data(population of over 550 000)..Actual values, inter/intradiurnal changes of followed parameters(State Meteorological Institute) .Intra/interdiurnal changes for every day(morning-evening) data. Expected incidence calculated(special formula,period of 10yrs).

Results: Multicenter study. Highest CI occurence foud in April, compared with other months and expected CI occurence. Possible explanation by highest meteorological activity. Significatly higher CI occurrence found at AtT-15 to $-20{ }^{\circ} \mathrm{C}$, AtP above 99, $32 \mathrm{kPa}$ copmpared with expected number of cases. AtSp, eAt of less significance. Actual meteorological values at given time needn't to be the most important factors. More important could be their inter-/intra-diurnal changes..Statistically highest frequency of CI at interdiurnal AtTdecrease, AtSp and AtP decline were found.. Favorable meteoroological situation represent interdiurnal AtT, AtSp and AtPincrease Relationship between $\mathrm{CI}$ occurrence and biometeorological relationship cannot be interpreted isolately from individual situation of organiism (IHD, AS, biological.functions etc.)...

\section{Conclusions:}

1. Significant correlations between seasonal variation, mainly in April and stroke incidence were found.

2. The same relationship between actual changes and inter-/ intradiurnal cahnges of four meteorological parameters ( mainly AtP, AtT) and incidence of stroke were found.

3. Interdiurnal decrease of AtT and AtP are predictors for $\mathrm{CI}$ onset

Supported by the gov.grants APVV-0586-06,LPP0186-06,MS-008KU-8/ 2008

Oral Presentations

Neuroepidemiology 2009;33:131-223 
SS13/5

\section{One Year Outcomes After Transient Ischaemic Attack (TIA): A Prospective Cohort Study Using Administrative Health Data}

Worthington J. ${ }^{1}$, Gattellari M. ${ }^{2}$, Aitken R. ${ }^{3}$, Jalaludin B. ${ }^{2}$, Cordato $D .^{1}$

${ }^{1}$ The University of New South Wales, South West Sydney Clinical School, Sydney, Australia, ${ }^{2}$ The University of New South Wales, School of Public Health and Community Medicine, Sydney, Australia, ${ }^{3}$ New South Wales Health, Sydney, Australia

Background: Reports of a high early stroke risk following TIA have prompted a review of TIA care. We aimed to determine stroke and death in the year following hospitalisation for TIA in a large contemporary cohort.

Methods: We identified hospitalised patients with a primary diagnosis of TIA (ICD-10, G45.x) from July 2004 to June 2005 within New South Wales, Australia (population $\sim 6.5$ million). Re-admissions to hospital with primary diagnosis of stroke (ICD10 I60.x-I64) were ascertained. Death data were obtained from the state death registry.

Results: We identified 3,464 TIA patients admitted to hospital (Mean age $=72.5$ years, $S D=13.3)$. Half $(n=1744,50.3 \%)$ were male. Almost half $(47.1 \%, \mathrm{n}=1633)$ had recognised hypertension, $13.0 \%(\mathrm{n}=451)$ atrial fibrillation and $15.0 \%(\mathrm{n}=520)$ diabetes. Within 30-days, 39 (1.1\%) were readmitted to hospital with a primary diagnosis of stroke. Of 134 patients $(3.9 \%)$ admitted for stroke within 12-months, $14.2 \%$ had haemorrhagic stroke, $44.8 \%$ ischaemic stroke, $39.6 \%$ were not otherwise specified and $1.5 \%$ could not be determined. Death at 30-days and 12-months occurred in $0.9 \%(\mathrm{n}=30)$ and $8.3 \%(\mathrm{n}=287)$ of patients, respectively. Atrial fibrillation $(\mathrm{OR}=1.6)$, diabetes $(\mathrm{OR}=1.5)$, increasing age $(\mathrm{OR}=1.1)$ and male sex $(\mathrm{OR}=1.4)$ independently and significantly predicted increased risk of death or stroke at 12-months, while recognized hypertension was associated with reduced risk $(\mathrm{OR}=0.74)$.

Conclusion: Early and one year risk of stroke was low in a cohort of TIA patients admitted to hospital, while death at one year was notably higher than stroke risk. The reduced risk with recognised hypertension may reflect improved outcomes of treatment.

\section{SS13/6}

\section{Ischaemic Strokes in Sri Lanka - Data from the Ragama Stroke Registry}

\author{
Ranawaka U.K.' , Amensi A. ${ }^{1}$, Wickramaratna C., \\ Pathmeswaran A. ${ }^{1}$, Kulatunga A. ${ }^{2}$, Tissera N. ${ }^{2}$, Cooray M. ${ }^{2}$, \\ Tetsuya M. ${ }^{3}$, Makaya M. ${ }^{3}$, Wickramasinghe $R$. \\ ${ }^{1}$ Faculty of Medicine, University of Kelaniya, Ragama, Sri \\ Lanka, ${ }^{2}$ Colombo North Teaching Hospital, Ragama, Sri \\ Lanka, ${ }^{3}$ International Medical Centre of Japan, Tokyo, \\ Japan
}

Background: Stroke events, disability and mortality are commoner in developing countries, but data are limited from these countries, especially from the South Asian region. We present data on ischaemic strokes from the first prospective Stroke Registry in Sri Lanka.

Methods: Information regarding all patients admitted with acute stroke are recorded in the Stroke Registry at the Colombo North Teaching Hospital, Ragama. We analysed data of patients admitted over a one-year period regarding demographic characteristics, delays in presentation, clinical features, stroke subtypes, risk factors, stroke severity and initial disability.

Results: Out of 547 patients, 457 (83.5\%) were ischaemic strokes. Mean age (SD) was 64.1 (12.8) years, $62.6 \%$ were $>60$ years, and $59.7 \%$ were males. A large number (41.2\%) presented to hospital within 3 hours of noticing symptoms.

Lacunar infarction was the commonest subtype (55.6\%). Hypertension (56.2\%) and diabetes (30.2\%) were the commonest risk factors. Obesity (based on waist circumference - Asian cutoffs) was common in women (48.4\%), but not in men $(15.9 \%)$. Smoking was very common among men $(69.2 \%)$, but rare in women (1\%). Atrial fibrillation was seen in $16.3 \%$. A history of stroke was noted in $13.6 \%$ and a positive family history in $23.6 \%$. Stroke severity and initial disability were high; NIHSS score $>7$ in $46.4 \%$, Barthel index $<60$ in $71.6 \%$, and modified Rankin score of $>2$ in $82.5 \%$.

Conclusions: Lacunar infarcts were commoner than in most reported series. Hypertension was the commonest risk factor identified. Many presented to hospital within 3 hours, indicating opportunity for acute interventions. 
SS14

Epidemiology of CNS Tumours and Infections

August 30, 2009

08:00-09:00

$\mathrm{SS} 14 / 1$

Intracranial Suppurations, a Severe but Preventable Disease: The Case of YaoundeCameroon

Djientcheu V.D.P. ${ }^{1}$, Lekoubou Looti A.Z. ${ }^{2}$, Njamnshi A.K. ${ }^{2}$, Nguefack S. ${ }^{3}$, Kamga Y. ${ }^{1}$, Esiene A. ${ }^{4}$, Kobela M. ${ }^{5}$

'Service of Neurosurgery Central Hospital Yaounde, Faculty of Medicine and Biomedical Sciences University of Yaounde I, Neurosurgery, Yaounde, Cameroon, ${ }^{2}$ Service of Neurology Central Hospital Yaoundé, Faculty of Medicine and Biomedical Sciences University of Yaounde I, Neurology, Yaounde, Cameroon, ${ }^{3}$ Service of Pediatric Neurology, Pediatrics Gynecological and Obstetrics Hospital Yaounde, Faculty of Medicine and Biomedical Sciences University of Yaounde I, Yaounde, Cameroon, ${ }^{4}$ Service of Anesthesiology Central Hospital Yaounde, Faculty of Medicine and Biomedical Sciences University of Yaounde I, Yaounde, Cameroon, ${ }^{5}$ Service of Pediatrics Central Hospital of Yaounde, Yaounde, Cameroon

Background: Although there is increasing recent evidence for a different pattern of intracranial suppurations between developed and developing countries, no data is available in Cameroon on this subject.

Patients and Methods: All files of patients operated for intracranial space occupying lesion in the Central Hospital of Yaounde, from January 2000 through December 2008 were reviewed for evidence of intracranial suppuration.

Results: Forty-one consecutive patients were recruited including 31 males (73.2\%). Intracranial suppurations represented $14.3 \%$ of all intracranial space occupying lesions. The median age was 11 years (extremes: 1 month and 73 years). Eight patients out of 10 were 20 years or younger. Clinical features included fever $(87.0 \%)$, headaches $(83.8 \%)$, altered consciousness $(81.0 \%)$, convulsions $(63 \%)$, vomiting $(30.3 \%)$, motor deficit $(23 \%)$ and speech disorders $(10.7 \%)$. The Bergman triad was frequent $(58.6 \%)$. Potential source of infection [absolute number (frequencies)] for intracranial suppuration were meningitis 9 (22), sinusitis $6(14.6 \%)$, head injury 5 (12.2), facial cellulitis $3(7.3)$, otitis 2(4.9) and endocarditis 1 (2.4). No source of infection was found in 11 cases $(26.8 \%)$. Empyema, abscess and pyoventriculitis represented respectively 27 (65.8\%), 12 (29.3\%) and $2(4.9 \%)$ cases. Burr hole $(88 \%)$ was the surgical approach of empyema. Abscesses were puncture through a burr hole in $72.7 \%$ of cases. Mortality and morbidity were $14 \%$ and $19.5 \%$ respectively. The long term cognitive impact of children needs to be investigated.
Conclusion: While its prevalence is dropping in developed countries, the burden of intracranial suppurations remains high in the developing world. Our data calls for urgent preventive actions.

\section{SS $14 / 2$ \\ The Austrian Brain Tumour Registry: A Novel Population-Based Brain Tumour Registry}

Wöhrer A. ', Waldhör T. ${ }^{2}$, Heinzl H. ${ }^{3}$, Hackl M. ${ }^{4}$, Feichtinger J. ${ }^{5}$, Gruber-Mösenbacher U. ${ }^{6}$, Kiefer A. ${ }^{7}$, Maier H. ${ }^{8}$, Motz R. ${ }^{9}$, Reiner-Concin A. ${ }^{10}$, Richling B. ${ }^{11}$, Idriceanu C. ${ }^{12}$, Scarpatetti M. ${ }^{13}$, Sedivy R. ${ }^{14}$, Bankl H.-C. ${ }^{14}$, Stiglbauer $W^{15}{ }^{15}$ Preusser $M .{ }^{16}$, Rössler $K^{17}{ }^{17}$, Hainfellner J.A. ${ }^{1}$

${ }^{1}$ Austrian Society of Neuropathology / Institute of Neurology, Medical University of Vienna, Vienna, Austria, ${ }^{2}$ Centre for Public Health, Department of Epidemiology, Medical University of Vienna, Vienna, Austria, ${ }^{3}$ Core Unit for Medical Statistics and Informatics, Section of Clinical Biometrics, Medical University of Vienna, Vienna, Austria, ${ }^{4}$ Austrian National Cancer Registry, Statistics Austria, Vienna, Austria, ${ }^{5}$ Department of Pathology, Krankenanstalt Rudolfstiftung, Vienna, Austria, ${ }^{6}$ Department of Pathology, Feldkirch State Hospital, Feldkirch, Austria, ${ }^{7}$ Institute of Pathology, State Hospital Klagenfurt, Klagenfurt, Austria, ${ }^{8}$ Department of Pathology, Medical University of Innsbruck, Innsbruck, Austria, ${ }^{9}$ Department of Pathology and Neuropathology, State Neuropsychiatric Hospital Wagner-Jauregg, Linz, Austria, ${ }^{10}$ Institute of Pathology, Danube Hospital, Vienna, Austria, ${ }^{11}$ Department of Neurosurgery, Christian Doppler Clinic, Paracelsus Private Medical University, Salzburg, Austria, ${ }^{12}$ Department of Neurology, Christian Doppler Clinic, Paracelsus Private Medical University, Salzburg, Austria, ${ }^{13}$ Institute of Pathology, Medical University of Graz, Graz, Austria, ${ }^{14}$ Department of Clinical Pathology, General Hospital St. Pölten, St.Pölten, Austria, ${ }^{15}$ Institute of Pathology, General Hospital Wr.Neustadt, Wr.Neustadt, Austria, ${ }^{16}$ Department of Internal Medicine I, Medical University of Vienna, Vienna, Austria, ${ }^{17}$ Task Force for Neurosurgical Oncology, Austrian Society of Neurosurgery, Feldkirch, Austria

Background: In Austria, registration of malignant brain tumours is legally mandatory, whereas benign and borderline tumours are currently not reported. The Austrian Brain Tumour Registry (ABTR) was initiated under the auspices of the Austrian Society of Neuropathology for the registration of malignant and non-malignant brain tumours.

Methods: All Austrian neurooncology units $(n=11)$ involved in brain tumour diagnostics contribute data on primary brain tumours. Non-microscopically verified cases are added by the Austrian National Cancer Registry to ensure a population-based dataset. Data analysis is performed in interaction with epidemiologists and biomedical statisticians.

Results: In 2005 we registered a total of 1,688 incident primary brain tumours in a population of 8,2 million inhabitants with an overall age-adjusted incidence rate of $18.1 / 100,000$ popula- 
tion/year. Non-malignant cases constituted 866 cases $(51.3 \%)$. The incidence rate was higher in females $(18.6 / 100,000)$ as compared to males $(17.8 / 100,000) .95 / 1,688(5.6 \%)$ cases were diagnosed in children $(<18$ years). The most common histology was meningioma $(n=504,29.9 \%)$ followed by glioblastoma $(n=340$, $20.1 \%)$, and pituitary adenoma $(\mathrm{n}=151,8.9 \%)$.

Conclusions: The ABTR model led by neuropathologists in collaboration with epidemiologists and the Austrian National Cancer Registry presents a novel way to establish a populationbased brain tumour registry. Cancer registration is linked to the mission of medical practice and research. International comparison of initial ABTR findings shows high congruency, thus confirming a high data quality. The continued operation of ABTR will enable monitoring incidence changes and identifying regional disease clusters or geographic variations in brain tumour morbidity/mortality.

\section{SS14/3 \\ High Prevalence of Intracranial Meningioma in Jewish Population in Shiraz, Southern Iran}

Taghipour M. ${ }^{1}$, Razmkon A. ${ }^{1}$, Bakhtazad A. ${ }^{2}$

${ }^{1}$ Department of Neurosurgery, Shiraz University of Medical Sciences, Shiraz, Iran, Islamic Republic of Iran, ${ }^{2}$ Shiraz University of Medical Sciences, Shiraz, Iran, Islamic Republic of Iran

Background: There are few well-established causes of intracranial tumors of the brain and nervous system among adults, and investigators have looked to associations between incidence and sociodemographic variables for clues to etiology. In this study, we tried to evaluate the relative risk of meningioma in Shiraz (Southern city of Iran) Jewish population.

Methods: A historical cohort study of adult intracranial meningioma was conducted in 2007 at five hospitals in Shiraz. Religion was recorded on admission in patients' charts, while an interview was done with every patient. To minimize the chance of missing even one Jewish patient, we contacted a few well-trusted Jewish family physicians. Chi square test was used to calculate relative risks.

Results: Shiraz mean population in the aforementioned period of time was approximately $1,255,955$, and mean Jewish population was 5784 people. There were 356 non-Jewish and 15 Jewish cases with intracranial meningioma. The relative risk for development of meningioma in Jewish population was 9.10 (95\% CI: 4.81-14.01; $\mathrm{P}<0.01$ ). The prevalence of meningioma in Jewish population in our series was 259 (95\% CI: 128-390) per 100,000 population.

Conclusion: Our study showed an increased risk of intracranial meningioma among those of Jewish religion. Meningioma risk was elevated almost 9 fold among Jewish residents of the city of Shiraz. Clearly-established environmental risk factors were not found to cause such a higher risk in this study. Our findings indicate the interference of genetic factors in the higher risk of meningioma among jews.

\section{LB-SS/SS14/4 \\ Fourteen-Year Mortality from Creutzfeldt- Jakob Disease in Cyprus}

Papacostas S., Kyriakides T.

The Cyprus Institute of Neurology and Genetics, Clinical Sciences, Nicosia, Cyprus

Creutzfeldt-Jakob disease (CJD) is a spongiform encephalopathy occuring in distinct etiologic subtypes; the sporadic form, with an incidence of about $0.5-1.5$ cases per million population per year, and constitutes approximately $85 \%$ of all CJD cases; iatrogenic forms that can be linked to contaminated surgical instruments or human tissue transplantation; familial forms which are associated with mutations of the prion protein gene (PRNP); and variant CJD (vCJD) which was first identified in 1996, occurs predominantly in the United Kingdom and was linked to bovine spongiform encephalopathy.

Suspected cases were referred to a focal point. WHO and EU collaborative study diagnostic criteria were used. Data were collected between 1995 and 2009. 11 cases were identified, one familial.

Determination of the presence of protein 14-3-3 in cerebrospinal fluid, brain MRI scans and EEG were carried out in all cases. Postmortem examination was performed in 2 cases. Patients were followed up closely until their death. PrP gene analysis was performed on 4/11 cases.

Two definite, 7 probable and 1 possible sporadic CJD cases were identified. One familial case was diagnosed. No vCJD cases were found in Cyprus during the period of surveillance.

All definite/probable cases had typical EEGs characterized by periodic sharp and slow wave complexes and were positive for protein 14-3-3. No significant factors or medical history were identified that would have increased the risk for CJD.

The adjusted incidence of CJD in Cyprus for the 14-year period of surveillance was 0.78 cases per million population per year. 
SS15

Stroke Epidemiology and Management (Part 6)

August 30, 2009

09:00-10:00

SS15/1 Marked Increase in Life Expectancy After
Stroke

Boysen G. ${ }^{1}$, Luis Marott J. ${ }^{2}$, Grønbæk M. ${ }^{3}$, Hassanpour H. ${ }^{1}$, Truelsen $T^{1}$

'Department of Neurology, Bispebjerg University Hospital, Copenhagen, Denmark, ${ }^{2}$ Copenhagen City Heart Study, Bispebjerg University Hospital, Copenhagen, Denmark,

${ }^{3}$ National Institute of Public Health, Copenhagen, Denmark

Background: Trends in life expectancy after stroke is available from only few studies. The present study presents changes in one-week stroke survivors from 1978-82 to 1998-2002.

Methods: Participants in the Copenhagen City Heart Study $(\mathrm{n}=19,698)$ who experienced a first-time-ever stroke from 1978 to end of 2001 were followed. Stroke events were validated using the World Health Organization's definition of stroke. Linkage to The National Register of Causes of Death provided data on time of death. Survival in stroke patients was compared with survival in the general population.

Results: Of 2,051 patients with first-ever stroke 1801 died during follow-up. Causes of death were cerebrovascular disease in $37 \%$, other cardiovascular diseases in $28 \%$, cancer in $12 \%$, and other causes in $23 \%$. For all age groups survival was poorer in stroke patients than in non-stroke controls. Long-term survival improved steadily over time. Life expectancy after stroke increased with up to 4 years from 1978 to end of 2001 exceeding the increase of life expectancy in the general population. Life expectancy in men and women aged 50 years increased from 13.2 years and 14.2 years in 1978-1982 to 17.2 years and 17.6 years in 1998-2002.

Conclusion: In this cohort life expectancy after stroke gradually improved over three decades from 1978. The gain in remaining lifetime after stroke exceeded that of the general population.

\section{SS15/2}

\section{Short-Term Prognosis and Hemorrhagic Transformation of Ischemic Stroke}

Terruso V. ${ }^{1}$, D'Amelio M. ${ }^{1}$, Sarno C. ${ }^{2}$, Famoso G. ${ }^{1}$, Di Benedetto N. ${ }^{1}$, Mazzola M.A. ${ }^{1}$, Ragonese P. ${ }^{1}$, Pinzone $F^{3}$, Aridon P. ${ }^{1}$, Savettieri G. ${ }^{1}$

${ }^{1}$ Università degli Studi di Palermo, Dipartimento di Neuroscienze Cliniche, Palermo, Italy, ${ }^{2}$ Università degli Studi di Palermo, Dipartimento di Biotecnologie Mediche e Medicina Legale, Palermo, Italy, ${ }^{3}$ AUSL 6, Servizio Dipartimentale di Sanità Pubblica, Epidemiologia e Medicina Preventiva, Palermo, Italy

Background: Little is known about mortality following spontaneous hemorrhagic transformation (HT) of ischemic stroke (IS).

Methods: We compared the mortality of individuals with spontaneous HT to that of individuals without HT. We reviewed medical records of patients diagnosed with anterior IS discharged from our Department during the period 2004-2006. Living status data were obtained from the public record office of the municipality of Palermo or directly by telephone interview. Outcome measure was 30 days survival after IS. HT was stratified in hemorrhagic infarction (HI) and parenchymal hematoma (PH).

Results: Of the 240 individuals with anterior IS we were able to determine living status for 233 individuals (97.1\%). A significantly higher number of patients died in the HT group $(\mathrm{n}=6$; $20.7 \%)$ compared to the non HT group $(n=13 ; 6.4 \%)(p=0.008)$. A highly significant difference was observed between the survival of the two groups $(\mathrm{p}<0.006)$. At univariate analysis presence of early CT signs (OR 2.9.0; 95\% CI 1.1, 7.5; p = 0.02), PH (OR $6.3 ; 95 \%$ CI $1.5,27.2 ; \mathrm{p}=0.01)$, and low level of consciousness at admission (OR 18.7; 95\% CI 3.8, 91.5; $\mathrm{p}=0.003$ ), were significantly associated with death. At multivariate analysis, only $\mathrm{PH}$ (OR 8.4; 95\% CI 1.8, 38.2; $\mathrm{p}=0.005)$ and low level of consciousness (OR 16.7; 95\% CI 3.2, 87.5; $\mathrm{p}=0.0009$ ) remained significantly associated with death.

Conclusions: Parenchymal hematoma and low level of consciousness at admission were associated with greater risk of death after acute ischemic stroke.

SS15/3

\section{Management of Stroke in Middle and East Europe}

Demarin V., Vuković V., Ažman D.

Department of Neurology, University Hospital 'Sestre milosrdnice', Zagreb, Croatia

Objectives: Epidemiological studies show that stroke incidence is higher in eastern countries and stroke is the leading cause of death and 30-day case fatality. The aim of this study was to analyze current management of acute stroke in 17 European countries: Austria, Belarus, Bosnia and Hercegovina, Bulgaria, Croatia, Czech Republic, Estonia, Georgia, Hungary, Italy, 
Kosovo, Lithuania, Montenegro, Romania, Russian Federation, Serbia and Slovenia.

Methods: A questionnaire has been sent to collaborating stroke centers.

Results: Stroke incidence is within the range of 183-623/100 000 inhabitants. All countries have stroke units, except Georgia, Montenegro and Kosovo. The majority of stroke patients are treated in Stroke units, followed by traditional neurological ward, and a small percentage of stroke patients are treated on other wards than neurological.

Availability of diagnostic procedures in stroke management largely depends on urban/rural setting and is as follows: computerized tomography (CT) scan is performed in $93-100 \%$ of all stroke cases; however CT is performed in 30-70\% within the first 24 hours after stroke admission. Duplex sonography is performed in $25-100 \%$ of all stroke patients.

Thrombolysis is being performed in most countries, it is still not available in Romania, Georgia, Montenegro and Kosovo. The number of patients treated with thrombolysis is increasing every year. After demission, $20-100 \%$ (on average 20-40\%) of patients is sent to a rehabilitation center.

Conclusions: The results of our study showed that most eastern European countries have a well developed neurological care system for acute stroke and most apply thrombolysis for stroke treatment.

\section{SS15/4 \\ The Evaluation of the First Croatian Stroke Unit at the 'Sestre Milosrdnice' University Hospital, Zagreb: 1995-2006 Experience}

Supanc V., Vargek-Solter V., Basic-Kes V., Breitenfeld T., Zavoreo I., Demarin V.

\section{Department of Neurology, University Hospital 'Sestre} milosrdnice', Zagreb, Croatia

Aim: To evaluate the effects of the first Croatian Stroke Unit (SU) by comparing the in-hospital case fatality rate in the period before (1995-2000) and after (2001-2006) the implementation of SU and to compare the prevalence of risk factors, such as hypertension, diabetes mellitus (DM), atrial fibrillation (AF) and ischemic heart disease (IHD) among the patients who died.

Patients and Methods: The study was conducted in twelveyear period during which 10,901 stroke patients were admitted to hospital and 1,818 of them died. The endpoints were in-hospital case fatality rate and prevalence of risk factors among the patients who died.

Results: Before the SU period the case fatality rate was $20.1 \%$, whereas afterwards it decreased to $12.8 \%$. The RR was 1.57 , while the estimate of the OR showed a $71 \%$ increase in chances of death in the pre-SU period. The prevalence of DM, IHD and AF increased significantly, while hypertension was the only risk factor which significantly decreased.

Conclusion: We confirmed that the implementation of SU care is associated with a significant reduction of in-hospital case fatality rate of acute stroke patients which strongly suggest that development of the SU network in Croatia should be given priority in the health management.

\section{SS15/5 \\ Early Seizures After Moderate/Severe Stroke: Risk Factors and Prognosis}

\author{
Guekht A. 1, Gulyaeva N. ${ }^{2}$, Khaimovsky I. ', Lebedeva A. ${ }^{1}$, \\ Hauser W.A. ${ }^{3}$, Gusev E. \\ ${ }^{1}$ Russian State Medical University, Neurology $\&$ \\ Neurosurgery, Moscow, Russian Federation, ${ }^{2}$ Institute of \\ Higher Nervous Activity and Neurophysiology RAS, \\ Moscow, Russian Federation, ${ }^{3}$ Columbia University, \\ Departments of Neurology, Epidemiology, and Sergievsky \\ Center, New York, USA
}

Background: Especially in the elderly population, stroke is the most common cause of seizures and epilepsy. About $10 \%$ of all stroke patients experience seizures, $5 \%$ are early seizures (ES, within 7 days of stroke) and another 5\% are late-onset seizures. Risk of seizures after stroke is higher in hemorrhage (HS) vs. ischemic stroke (IS). Still data about risk factors and prognosis of ES are rather contradictory.

Methods: A prospective single-center study of 1427 patients (863 IS, 564 HS) with moderate/severe first-ever stroke, admitted to intensive care unit of the Neurology department.

Results: ES occurred in $91(6.3 \%)$ of patients, including 40 $(4.6 \%)$ with IS and $51(9.6 \%)$ with HS. Of all ES $67 \%$ in IS and $63 \%$ in HS occurred within the first day of stroke. ES were secondarily generalized in the majority of cases. The risk of ES (odds ratio, $95 \%$ confidence limits) in IS was $5.56(2.9-10.65)$ for history of myocardial infarction, 5.38 (2.48-11.65) for diabetes mellitus, 3.77 (1.38-10.32) for atrial fibrillation. For patients with HS - 2.9 (1.61-5.18) for diabetes mellitus, $2.17(0.99-4.75)$ for history of alcohol abuse. Forty-five percent of patients with IS and ES had cardioembolic stroke vs. $8 \%$ with IS without ES ( $p<0.0001)$. Mortality of patients with ES during the first week was $45 \%$ (for IS), $70.5 \%$ (for HS), both significantly higher ( $p=0.005$ for IS and $\mathrm{p}=0.04$ for HS) vs. patients without ES.

Conclusions: ES occur mostly within the first days after stroke and constitute an important risk factor for in-hospital mortality.

$\overline{168} \quad \overline{\text { Neuroepidemiology 2009;33:131-223 }}$

1st International Congress on Clinical Neurology and Epidemiology, Munich 2009 


\section{SS15/6 \\ High Incidence of Stroke in Iran: the Mashhad Stroke Incidence Study (MSIS)}

Azarpazhooh M.R. ${ }^{1}$, Eatemadi M.M. ${ }^{1}$, Majdi M.R. ${ }^{2}$, Mokhber N. ${ }^{3}$, Ghayour-Mobarhan M. ${ }^{4}$, Ghandehary K. ${ }^{1}$, Farzadfard M.T. ${ }^{1}$, Kiani R. ${ }^{1}$, Panahandeh M. ${ }^{1}$, Thrift A. G. ${ }^{5}$

${ }^{1}$ Department of Neurology, Ghaem Hospital, Mashhad University of Medical Science, Mashhad, Iran, Islamic Republic of Iran, ${ }^{2}$ Department of Health, Mashhad University of Medical Science, Mashhad, Iran, Islamic Republic of Iran, ${ }^{3}$ Department of Psychogeriatric and Psychiatry, Avecina Hospital, Mashhad University of Medical Science, Mashhad, Iran, Islamic Republic of Iran, ${ }^{4}$ Department of Nutrition and Biochemistry, Mashhad University of Medical Science, Mashhad, Iran, Islamic Republic of Iran, ${ }^{5}$ Baker IDI Heart \& Diabetes Institute, Stroke Epidemiology, Melbourne, Australia

Background: Most incidence studies of stroke have been performed in western countries and incidence rates in these regions are unlikely to apply to developing regions such as Iran. We aimed to determine stroke incidence in Mashhad, North-East of Iran.

Methods: We used multiple overlapping sources to prospectively identify all strokes occurring in 450,229 residents during a 12-month period of 2006-2007. A large number of Community Health Volunteers identified people with stroke who were not admitted to hospital. Potential cases were reviewed by a group of stroke experts before inclusion.

Results: A total of 624 first-ever strokes occurred during the study period. Imaging was performed in $98.4 \%$ of patients. The crude annual stroke incidence rate was 139 (95\% CI 128-149) per 100,000 residents. When adjusted to the European population 45 to 84 years stroke incidence was considerably greater than in most other countries: 616 (95\% CI 567-664) for ischaemic stroke, 94 (95\% CI 75-113) for intracerebral haemorrhage and 12 (95\% CI 5-19) for subarachnoid haemorrhage. When age-specific incidence rates of ischaemic stroke were compared to those of western countries, ischaemic stroke in Mashhad was found to occur approximately one decade earlier.

Conclusions: The results of this study provide evidence that the incidence of stroke in Iran is considerably greater than in most western countries, with stroke occurring at younger ages. The incidence of ischaemic stroke was also greater than reported in other regions.

\section{SS16 \\ Translational Neurogenetic- Neuroepidemiology Research \\ August 30, 2009 \\ 09:00-10:00}

\section{SS16/1 \\ Orexin (Hypocretin) Gene Transfer in Narcolepsy}

Shiromani P. ${ }^{1}$, Liu M. ${ }^{2}$, Blanco-Centurion C. ${ }^{2}$

1 VA Boston Healthcare System and Harvard Medical School, Neurology, West Roxbury, USA, ${ }^{2}$ Harvard Medical School, Neurology, West Roxbury, USA

Narcolepsy is a neurodegenerative disorder linked to the loss of orexin neurons. A behavioral phenotype that resembles narcolepsy occurs in mice when the orexin gene or the orexin neuron is deleted. Gene transfer has proven to be an effective neurobiological tool in a number of neurodegenerative diseases but it is not yet known if it can also correct a sleep disorder. We have constructed two viral vectors to transfer the gene for mouse preproorexin to reverse the symptoms of narcolepsy in orexin knockout and orexin-ataxin mice.

In-vitro tests (RT-PCR and immunohistochemistry) confirmed expression of the transferred orexin gene into cultured cells. The vector was delivered into the lateral hypothalamus $(\mathrm{LH})$ of orexin knockout $(\mathrm{n}=13)$ and orexin-ataxin mice $(\mathrm{n}=8)$ and sleep behavior assessed. Control mice $(\mathrm{n}=9)$ received vector carrying only the reporter gene (GFP).

Numerous orexin-A immunoreactive neurons were observed in the LH of orexin knockout and orexin-ataxin mice upto 36 days after gene transfer. Orexin-A was detected in the CSF indicating release of the peptide. In the orexin null mice orexin gene transfer into the LH decreased the incidence of cataplexy by $60 \%$ (versus control vector), and the levels of REM sleep during the second half of night were same as WT. Sleep data analysis is still not completed in the orexin-ataxin mice given the orexin gene.

This is the first study to demonstrate that gene transfer can improve symptoms of narcolepsy in an animal model of narcolepsy. Supported by NIH grants (NS030140, NS052287, HL91363) and VA Research Service 


\section{SS16/2}

\section{Influence on Repair of Neural Lesions by Expression of VEGF Following Crush Injuries of Sciatic Nerves in Rats}

Tang J., Ding $X$.

Nanjing Medical University, Neurology, Nanjing, China

Background: By recent researches, it was suggested that vascular endothelial growth factor (VEGF) had protective property to lesions of neurons or nerves in ischaemia of brain, disorder of spinal cord, and even impairment of peripheral nerve.

Methods: Sciatic nerve was crushed by pincers to establish the model of sciatic nerve crush lesion in rats. All of 320 SD rats were divided into control group, saline-treated group, alprostadil group and buflomedil group at random. SPSS 11.5 was used in statistical analysis. By means of RT-PCR, immunohistochemistry, electromicroscope and electrophysiology, the expression of VEGF of neurons in dorsal root ganglia (DRGs) and the changes of neural lesions and neural functions following crush injury of sciatic nerves were studied. The effects of two drugs of improving microcirculation, alprostadil and buflomedil, on expression of VEGF, repair of neural lesions and recovery of neural functions were also examed.

Results: We found that the expression of VEGF and the number of positive VEGF neurons significantly increased in alprostadil and buflomedil groups. Besides, these substances caused less pathological changes in DRGs, improvement of nerve conduction velocities (NCVs) of sciatic nerve and increase of toe spaces.

Conclusions: Our results indicate that the improvement of microcirculation may reduce the injury of peripheral nerve, improve the repair of the neural function following crush injury of peripheral nerve, which may have a relationship with the increase of expression of VEGF.

\section{SS16/3 \\ Effect of Brain Derived Neurotrophic Factor RNA Interference on Dopaminergic PC12 Cell Apoptosis}

Ding $X$.

Nanjing Medical University, Neurology, Nanjing, China

Objective: The exact role of brain derived neurotrophic factor (BDNF) in pathogenesis of Parkinson's disease (PD) was poorly understood. The aim of the present study was to explore the effect of BDNF RNA interference (RNAi) on dopaminergic PC12 cell apoptosis.

Methods: Specific small double-stranded interfering RNA (siRNA) targeting BDNF mRNA was transfected into PC12 cell line with or without BDNF protein protection. The expression of $\mathrm{BDNF}$ andBax/Bcl-2 mRNA were measured by real time PCR. Immunofluorescence was performed to analyze the expression of BDNF protein. Lactate dehydrogenase (LDH) release was assayed for determining neurotoxicity. Cell apoptosis was detected by Annexin V/PI double staining.
Results: The expression of BDNF mRNA reduced over $70 \%$ in the transfected cells as compared with the control group, but not in the scrambled siRNA transfected group. BDNF RNAi induced LDH release and cell apoptosis through bax signal pathway, which was in accord with 6-Hydroxydopamine (6-OHDA) induced neurotoxicity. However, cell apoptosis was alleviated when incubated with BDNF protein.

Conclusion: The present study suggested that BDNF RNAi could induce apoptosis of PC12 cells via bax pathway, which partly mimics 6-OHDA-induced neurotoxicity. BDNF may be a prospective therapeutic agent for treatment of PD.

\section{SS16/4 \\ Effect of Aqueous Extract of Valerian Officinalis Root on PTZ-Induced Seizure in Mice}

\author{
Namvaran A. ${ }^{1}$, Khayat Nouri M.H. ${ }^{2}$, Golabi M. ${ }^{1}$ \\ ${ }^{1}$ Islamic Azad University, Student of veterinary medicine, \\ Islamic Azad University Tabriz branch, and member of \\ Young Researchers Club, Tabriz-Iran, Pharmacology, \\ Tabriz, Iran, Islamic Republic of Iran, ${ }^{2}$ Islamic Azad \\ University, Department of Basic Science (Pharmacology), \\ Tabriz, Iran, Islamic Republic of Iran
}

Introduction: Phamacognostic studies shown that the root of valerian officinalis (VO) contains gamma-amino butyric acid and other materials. In traditional medicine anticonvulsant effect of VO is reminded. number of studies have been shown that this herb has anticonvulsant and hypnotic effects in a some of animal seizure models.

The aim of this study was to investigate the effect of aqueous extracts of VO on PTZ-induced clonic seizure threshold in mice.

Material and Methods: After determination of the intravenously PTZ-induced seizure threshold in control group, extract of valerian officinalis root $(0.25,0.5$ and $1 \mathrm{~g} / \mathrm{kg})$ orally administration and investigate the effects of VO on clonic seizure threshold.

Results: Our results shown that the PTZ-seizure threshold was in control group in animals pretreated with VO the PTZ seizure threshold dose dependently increased, significantly $(\mathrm{P}<$ 0.05).

Conclusion: Our results suggest that VO posse's anticonvulsant activity probably is via GABAergic effect. 


\section{SS16/5 \\ Effect of Rehabilitation Training on Nerve Regenerative Microenviroment After Spinal Cord Injury of Rats}

Lei $X$., Ding $X$.

Nanjing Medical University, Neurology, Nanjing, China

Background: Since Aguayo etc has proved that central nervous system has certain degree of regeneration after spinal cord injury, treatment pathway has changed from neural protection to promote nerve regeneration, namely the promotion of survival or replacement of the injuried neurons and supporting cells

Methods: Sciatic nerve was crushed by pincers to establish the model of sciatic nerve crush lesion in rats. All of $320 \mathrm{SD}$ rats were divided into control group, saline-treated group, alprostadil group and buflomedil group at random. SPSS 11.5 was used in statistical analysis. By means of RT-PCR, immunohistochemistry, electromicroscope and electrophysiology, the expression of VEGF of neurons in dorsal root ganglia (DRGs) and the changes of neural lesions and neural functions following crush injury of sciatic nerves were studied. The effects of two drugs of improving microcirculation, alprostadil and buflomedil, on expression of VEGF, repair of neural lesions and recovery of neural functions were also examed.

Results: We found that the expression of VEGF and the number of positive VEGF neurons significantly increased in alprostadil and buflomedil groups. Besides, these substances caused less pathological changes in DRGs, improvement of nerve conduction velocities (NCVs) of sciatic nerve and increase of toe spaces.

Conclusions: Our results indicate that the improvement of microcirculation may reduce the injury of peripheral nerve, improve the repair of the neural function following crush injury of peripheral nerve, which may have a relationship with the increase of expression of VEGFB

\section{LB-SS/SS16/6 \\ Personalised Modelling for Risk and Outcome Prognosis on a Case Study of Brain Disease}

Kasabov N. ${ }^{1}$, Hu Y. ${ }^{1}$, Liang L. ${ }^{2}$

${ }^{1}$ Auckland University of Technology, KEDRI, Auckland, New Zealand, ${ }^{2}$ Auckland University of Technology, Auckland, New Zealand

Despite the furore of interest in this area, there are at present no adequate data analysis methods and systems which can produce highly accurate and informative personalised models from data, especially related to complex brain diseases $[1,2]$.

The talk presents a new methodology for personalized modelling [5] and illustrates it on a case study problem of central nervous system (CNS) diseases response to treatment based on a large number of personalised genetic and clinical data. The presented method includes: (1) selection and ranking of important personal variables and samples in relation to a problem scenario;
(2) creation of a personalised classification/prognostic model using brain-inspired computational techniques called neural networks, and more specifically - spiking neural networks (SNN); (3) design of a personalised profile and improvement scenarios.

When applied on the CNS case study problem data, the method not only achieved a better accuracy ( $85 \%$, compared with the $78.3 \%$ in [3]), but produced a personalised profile for each patient that can be used for a potential personalised treatment and drug design.

The method can be applied on many other problems, specifically brain disease risk and outcome prognosis, where personalised data is available.

\section{References}

[1] N. Kasabov (2007) Evolving Connectionist Systems: The Knowledge Engineering Approach, Springer.

[2] N. Kasabov, Global, local and personalised modelling and profile discovery in Bioinformatics: An integrated approach, Pattern Recognition Letters, Vol. 28, 6, April 2007, 673-685.

[3] Pomeroy, S., Tamayo, P., et al.: Prediction of central nervous system embryonal tumour outcome based on gene expression. Nature 415 (2002), 436-442.

SS17

Epidemiology of Dementia and Other Cognitive Disorders

August 30, 2009

10:30-11:30
Background: Although Mild Cognitive Impairment (MCI) represents a high-risk factor for developing dementia, little is known about the epidemiology of MCI among patients of General Practitioners (GP). Thus, the aim of the study was to provide 
prevalence and incidence rates of MCI among elderly GP patients and to identify underlying risk factors.

Methods: Data were derived from the longitudinal German Study on Ageing, Cognition and Dementia in Primary Care Patients (AgeCoDe). Altogether, 3,327 GP patients aged 75+ were assessed by structured clinical interviews in a baseline and two follow-up waves. Prevalence of MCI at baseline was estimated as the percentage of the completely assessed non-demented subjects. Incidence was calculated according to the 'person-years-at-risk' method. Risk factors were analysed using multivariate logistic regression models.

Results: Prevalence of MCI was $15.4 \%(95 \%-\mathrm{CI}=14.1$ 16.6). Positive associations were found for older age (85+), apoE $\varepsilon 4$ allele, stroke and depressive symptoms. During the 3-year follow-up period, additional 350 patients developed MCI (person-years $=6198.20)$. The overall incidence of MCI was 56.5 $(95 \%$-CI $=50.7-62.7)$ per 1,000 person-years. Older age, vascular diseases and the apoE $\varepsilon 4$ allele were also identified as significant risk factors for future MCI. Furthermore, a higher risk was found for patients with subjective memory complaints.

Conclusions: MCI is frequent in older GP patients and should be followed with regard to an early detection of incipient cognitive deterioration up to dementia. Subjective complaints should be taken seriously for this early detection. Especially vascular risk factors provide the opportunity of preventive approaches.

\section{SS17/2 \\ Are There Specific Psychological and Behavioural Symptoms of Depression in Dementia Patients?}

\author{
Prado-Jean A. ${ }^{1}$, Nubukpo P. ${ }^{2}$, Couratier P.1, Bernard- \\ Bourzeix L. ${ }^{1}$, Dechamps N. ${ }^{1}$, Dantoine T. ${ }^{1}$, Clément J.P. ${ }^{1}$ \\ ${ }^{1}$ Research Memory Center of Limousin, Limoges, France, \\ ${ }^{2}$ Institute of Epidemiology and Tropical Neurology, \\ Limoges, France
}

Background: Psychological and behavioural symptoms in dementia (BPSD) are very frequent, so that $95 \%$ of patients have at least one. Third of persons with dementia have depressive symptoms, and concomitant BPSD are very frequent.Objective. The study aimed to characterize these psychological and behavioural manifestations of depression in patients with dementia.

Methods: We recruited 331 patients with dementia from several nursing homes of Limousin-France. The depression was diagnosed by the Cornell Scale for depression in dementia (CSDD) with a cut-off of 7, and the BPSD were assessed using Neuropsychiatric inventory (NPI).

Results: Of 331 individuals with dementia, 42.9\% $(n=142)$ had depression and 75.8\% $(n=251)$ had BPSD. All BPSD were significantly $(\mathrm{p}<0.0001)$ more present in depressed patients. But we did not have significantly difference for sex and age. The most common NPI symptoms in depressed patients other than depression were agitation (43.7\%), anxiety $(42.9 \%)$, apathy $(41.5 \%)$ and irritability (40.8\%). Four NPI-based factors were indentified ( $60.8 \%$ of the common variance): environmental factor (irritability, disinhibition, agitation), positive factors (elation, hallucination, delirium), emotional factor (anxiety, apathy, appetite disturbance) and circadian factor (aberrant motor behavior, sleep disturbance). The depression in dementia patients was significantly associated with environmental, positive and emotional factors.

Conclusion: BPSD are a common and major problem. Before to consider them as isolated symptoms, this is important to assess co-morbidity with depression in order to have better therapeutic attitudes.

\section{SS17/3 \\ Angina Pectoris Severity and Functional Class Among Coronary Patients Predict Poorer Cognitive Function}

\author{
Weinstein G. ${ }^{1}$, Goldbourt U. ${ }^{1}$, Schwartz R. ${ }^{2}$, Phillips T. ${ }^{1}$, \\ Tanne D. ${ }^{2}$ \\ ${ }^{1}$ Department of Epidemiology and Preventive Medicine, \\ Tel-Aviv University, School of Public Health, Sackler Faculty \\ of Medicine, Tel-Aviv, Israel, ${ }^{2}$ Sheba Medical Center, \\ Neuroscience Center and Department of Neurology, Tel- \\ Hashomer, Israel
}

Background: The relationship between coronary heart disease (CHD) and cognitive impairment is not yet elucidated, and atherosclerosis has been suggested as an underlying pathological process.

Methods: We examined the association between severity of angina pectoris and its accompanying characteristics and subsequent cognitive dysfunction among a subgroup of chronic CHD patients, who previously participated in a secondary prevention trial. Severity of angina was assessed at baseline (1990-1992) by the Canadian Cardiovascular Society angina classification, and functional capacity by the New-York Heart Association (NYHA) classification. Cognitive scores were assessed $15 \pm 3$ years later, using a validated set of computerized cognitive tests (Mindstreams Computerized Cognitive Battery; computing index scores summarizing performance in each cognitive domain and a global cognitive score). We compared means of cognitive scores normalized to age and education between severity classes, and performed linear regression models adjusting for potential confounders.

Results: Among 346 patients (mean age at cognitive assessment $72 \pm 6$ yrs, $95 \%$ males, $19 \%$ diabetics), global cognitive score was $95.3 \pm 11.5$ in patients without active angina at baseline and $94.7 \pm 10.8$ and $89.7 \pm 11.5$ in patients with angina classes 1 and $\geq 2$, respectively $(\mathrm{p}=0.003)$. The mean global score of patients with NYHA class $\geq 2$ was lower than of patients with class $1(90.1 \pm 11.4$ vs. $95.1 \pm 11.4, \mathrm{p}=0.002)$. Similar significant differences were observed for specific cognitive domains, namely memory, executive functions, attention, and for standard clock-drawing and Mini-Mental tests. These associations remained significant after adjusting for potential confounders.

Conclusions: In patients with pre-existing CHD, angina severity and functional class are associated with late-life poorer cognitive functions. 


\section{SS17/4 \\ Prevalence of Dementia and Cognitive Disorders in Elderly Living in Central Africa: Bangui and Brazzaville}

Guerchet M. ${ }^{1}$, M'Belesso P. ${ }^{2}$, Mouanga A.M. ${ }^{3}$, Bandzouzi B. ${ }^{4}$, Houinato D. ${ }^{5}$, Paraïso M.N. ${ }^{6}$, Aboyans V. ${ }^{7}$, Nubukpo P. ${ }^{6}$, Clément J.P. ${ }^{8}$, Preux P.M. ${ }^{6}$

${ }^{1}$ Université de Limoges, IFR 145 GEIST, Institut d'Epidémiologie Neurologique et de Neurologie Tropicale, EA3174 NeuroEpidémiologie Tropicale et Comparée, Limoges, France, ${ }^{2}$ Service de Neurologie de l'Hôpital de I'Amitié, BP 3183, Bangui, Central African Republic, ${ }^{3}$ Service de Psychiatrie, CHU de Brazzaville, BP32, BP32, Brazzaville, Congo, ${ }^{4}$ Service de Neurologie, $\mathrm{CHU}$ de Brazzaville, BP32, Brazzaville, Congo, ${ }^{5}$ Université d'Abomey Calavi, Faculté des Sciences de la Santé, Unité d'Enseignement et de Recherche de Neurologie, Cotonou, Benin, ${ }^{6}$ Université de Limoges, IFR 145 GEIST, Institut d'Epidémiologie Neurologique et de Neurologie Tropicale, EA3174 NeuroEpidémiologie Tropicale et Comparée, Limoges, France, ${ }^{7} \mathrm{CHU}$ Limoges, Service de Chirurgie Thoracique Cardiovasculaire et Angiologie, Limoges, France, ${ }^{8} \mathrm{CHU}$ Limoges, $\mathrm{CH}$ Esquirol, Centre Mémoire de Ressources et de Recherche, Limoges, France

Background: Dementia and related syndromes are of major public health concern because of the ageing of global population. Few researches have been carried out in developing countries, especially in Africa where data are scarce compared to developed countries.

Methods: Two epidemiological population surveys on dementia were performed in Central Africa (Central African Republic and Republic of Congo). Door to door surveys were carried out in districts of Bangui and Brazzaville. Participants aged $>65$ years old were interviewed for sociodemographical data, medical history and depressive disorders. The CSI-D cognitive tests were conducted. Subjects who had a poor performance $(<$ 25.5/30) at the cognitive section of the CSI-D or had less than ten at the Five-Word Test were clinically assessed by a neurologist. Further psychometrical tests were conducted as Free and Cued Selective Reminding Test, Isaacs Set Test, Zazzo's cancellation test. The DSM-IV and NINCDS-ADRDA criteria were required for dementia and Alzheimer's disease diagnoses.

Results: Among the 501 individuals interviewed in Bangui, $188(37,5 \%)$ subjects suspected of cognitive impairment or dementia, 165(32,9\%) were re-examined by the neurologist. Among the 520 participants interviewed in Brazzaville, 148(28, 5\%) were suspect for cognitive impairment or dementia. Confirmation of cases is in progress but estimations of prevalences in both countries seems to be under $10 \%$. Prevalences and clinical description will be presented for both sites.

Conclusion: These results will add to the few available figures from developing countries. Comparison of prevalences with other studies, in particular in developed countries, could lead to new physiopathological hypotheses.

\section{SS17/5 \\ Cognitive Impairment in Elderly Women and Fertile Life Characteristics. A Population Based Study in Sicily}

Ragonese P., Aridon P., Mazzola M.A., D'Amelio M., Salemi G., Savettieri G.

Dipartimento Universitario di Neuroscienze Cliniche, Università degli Studi di Palermo, Palermo, Italy

Background: Previous studies indicated gender differences in rates of cognitive impairment.

Methods: We performed a door-to-door population-based study in the city of Bagheria, Sicily (prevalence day September, $30^{\text {th }} 2006$ ). A cohort of 1195 women was randomly stratified by age, and city wards, obtaining a $25 \%$ sample of the whole women population aged 65 years or older. Cognitive function was assessed in the first phase using Mini-Mental State Examination (MMSE). Cognitive impairment was measured according to several cut-offs $(26,24,22)$ corresponding to percentiles of distribution in the studied population $\left(50^{\circ}, 30^{\circ}, 25^{\circ}\right)$. Linear and logistic regression models were also used to analyze the relationship between cognitive performance and characteristics of fertile life (age at menarche, age at menopause, fertile life duration, live birth pregnancies and abortions) by means of a structured face-to-face questionnaire.

Results: 1195 women were included, 1072 agreed to participate (84\%). Distribution of MMSE calculated as a continuous variable or stratified by percentile among gender showed a highly significant inverse linear trend related to the increasing age $(p<0.012)$, and total duration of pregnancies $(p<0.010)$. Logistic regression models showed a significant trend for the association between cognitive impairment and surgical menopause; the OR for the association between cognitive impairment with MMSE less than 24 and surgical menopause was 0.25 ; CI $=0.09-0.69$; $\mathrm{p}=0.008$.

Conclusion: These findings suggest and association between cognitive impairment and characteristics of fertile life in elderly women.

\section{LB-SS/SS17/6 \\ Local Differences in Stroke Management on the Basis of Polish National Stroke Registry}

Ryglewicz D. ${ }^{1}$, Lechowicz W. ${ }^{2}$

${ }^{1}$ Ist Department of Neurology, Institute of Psychiatry and Neurology, Warsaw, Poland, ${ }^{2}$ Institute of Psychiatry and Neurology, Warsaw, Poland

Background: In Poland epidemiological studies show differences regarding stroke mortality.

The aim of the study was to evaluate differences in delay of hospital admission time, availability of diagnostic procedures, percentage of patients treated in Stroke Units and the percentage of intravenous thrombolysis (IVT). 
Methodology: Polish National Stroke Registry is conducted in cooperation between Polish National Health Fund and the Institute of Psychiatry and Neurology under the auspicious of Polish National Project of Cardiovascular Disease (POLKARD). The data of all hospitalized patients with stroke are registered online in the form of unified questionnaires in all 16 districts of Poland.

Results: From January $1^{\text {st }}$ to December $31^{\text {st }} 2008$ data from 61,119 patients $(52.7 \%$ women, mean age 75.1 , and $47.3 \%$ men, mean age 68.7) were collected into the database. Regional hospitalization rates ranged from $162.1 / 100,000$ to $247 / 100,000$, admissions to hospital during the first 3 hours of stroke from $3 \%$ to $12.5 \%$. Ischemic strokes were diagnosed in $78 \%-86 \%$, primary intracerebral hemorrhage strokes in $9 \%-11 \%$, subarachnoid hemorrhage in $2 \%-3.5 \%$ and unclassified stroke in $2 \%-11 \%$ of patients. CT was performed in $92 \%-98.5 \%$, carotid USG in some districts only in $32 \%-44 \%$, vs. $82 \%-86 \%$ in others. Significant differences have been found in the percent of patients treated in Stroke Units, from $7.4 \%$ to $49 \%$. Among those IVT was performed in $0.5 \%$ $9.8 \%$. In-hospital fatality was $14.5 \%-26.4 \%$.

Conclusion: The results of our study demonstrate a positive trend in stroke outcome in some Polish districts most likely as the result of better management of acute stroke.

SS18

Stroke Epidemiology and

Management (Part 7)

August 30, 2009

10:30-11:30

\section{SS18/1 \\ Early Development of Spasticity Following Stroke: A Prospective, Controlled Trial

\begin{abstract}
Wissel J. ${ }^{1}$, Schelosky L. ${ }^{2}$, Scott J. ${ }^{1}$, Christe W. ${ }^{3}$, Faiss J. ${ }^{4}$, Wisse/ J. ${ }^{5}$

${ }^{1}$ Kliniken Beelitz GmbH, Beelitz-Heilstätten, Germany, ${ }^{2}$ Kantonsspital Münsterlingen, Münsterlingen,

Switzerland, ${ }^{3}$ Ernst von Bergmann Klinikum, Potsdam, Germany, ${ }^{4}$ Asklepios Fachkliniken Brandenburg, Teupitz, Germany, ${ }^{5}$ Vivantes Spandau, Berlin, Germany
\end{abstract}

Background and Purpose: Spasticity is one of the positive phenomena following upper motor neuron (UMN) lesions. Spastic muscles develop changes in their rheologic properties, contracture, fibrosis, and atrophy. The functional changes in the affected limbs may cause pain, dependency, and decline in the quality of patient's life and self esteem. Despite the high public burden of the sequels of UMN lesions little is known about the time course and risk factors for development of post-stroke spasticity.
Methods: This study closely followed a cohort of 103 patients at median 6 days, 6 and 16 weeks after stroke and recorded muscle tone (modified Ashworth scale score = MAS), pain, paresis, Barthel Index and quality of life score (EQ-5D).

Results: $24.5 \%$ of stroke patients developed an increase of muscle tone within two weeks after stroke. Patients with spasticity had significantly higher incidences of pain and nursing home placement and lower Barthel and EQ-5D scores than patients with normal muscle tone. Early predictive factors for presence of severe spasticity (MAS $\geq 3$ ) at final follow up were moderate increase in muscle tone at baseline and/or first follow up (MAS = 2), low Barthel Index at baseline, hemispasticity and involvement of more than 2 joints at first follow up, and paresis at any assessment point.

Conclusions: Increase in muscle tone develops very early after stroke. The study helps to identify patients at highest risk for permanent and severe spasticity, and advocates for early treatment in this group.

\section{SS18/2 \\ Seizures in Patients with Symptomatic Cervical Artery Occlusion by Dissection and by Atherosclerosis}

De Reuck J. ${ }^{1}$, Van Maele G. ${ }^{2}$

${ }^{1}$ Department of Neurology, University Hospital, Ghent, Belgium, ${ }^{2}$ Department of Medical Statistics, University Hospital, Ghent, Belgium

Background: The outcome of patients with occlusion by cervical dissection (OCD) is considered to be better than of those with atherosclerotic occlusion of cervical arteries (ATO). The present observational retrospective study investigates whether there are also differences in the occurrence of seizures between OCD and ATO patients.

Patients and Methods: The characteristics and the occurrence of seizures in 199 consecutive patients with a stroke due to a cervical artery occlusion are determined. Forty patients with OCD and 159 with ATO are compared.

Results: OCD patients are significantly younger and have less vascular risk factors than those with ATO. Stroke type, vascular territory, NIHS score and outcome are similar. Three $(7.5 \%)$ OCD and 29 (18.2\%) ATO patients developed seizures. However, this difference is not statistically significant $(\mathrm{P}=0.146)$. On multiple logistic regression analysis ageing and arterial hypertension are independent variables.

Discussion: The present study suggests a lower incidence of seizures in patients with OCD compared to those with ATO, although not statistically proven, due to the small sample size. Age and arterial hypertension can explain this difference. 


\section{SS18/3 \\ Short Term and Long Term Mortality After Stroke in Northwest of Iran}

Aminisani N. ${ }^{1}$, Savadi Oskuei D. ${ }^{2}$, Shamshirgaran S.M. ${ }^{3}$, Dastgiri S. ${ }^{4}$, Hashemilar M. ${ }^{2}$

${ }^{1}$ Tabriz University of Med/Sci. University of Sydney, Epidemiology, Sydney, Australia, ${ }^{2}$ Tabriz University of Med/Sci., Neurology, Tabriz, Iran, Islamic Republic of Iran,

${ }^{3}$ University of Western Sydney, Medicine, Sydney,

Australia, ${ }^{4}$ Tabriz University of Med/Sci., Community

Medicine, Tabriz, Iran, Islamic Republic of Iran

Background: There is no strong evidence related to survival after Stroke and related risk factors in Iran. The aim of this study was to analysis the short-term and long-term survival in first-ever stroke population in Ardabil province/North Western of Iran.

Methods: patients with first-ever stroke were recruited and followed up to evaluate short-term and long-term survival From 21 Feb. 2004 to 22 Dec. 2006. We prospectively recorded all cases of stroke (defined according to the ICD-10) in residents of Ardabil province who admitted to only referral hospital for Stroke in the capital city of this province. Initial Information about risk factors, type of stroke, and its clinical futures for every patient was collected by trained interviewers (one nurse and one medical student) in the hospital and after 1, 3 months, and 1 year, the information related mortality were completed by selected interweavers from health centres through the home visits.

Results: A total of 352 patients diagnosed with stroke included in the study and 328 of them completed the study for 1n year. $81.8 \%$ ischemic stroke and others had been diagnosed with intra-cerebral hemorrhagic or subarachnoid haemorrhage. 28 days case fatality rate was $20.3 \%, 24 \%$ died after 3 months and $30.8 \%$ after 1 year which was significantly higher in hemorrhagic stroke compared to ischemic stroke. Age, type of stroke and cardiac disease were the most important predictors of mortality after stroke.

Conclusion: There is a need to implement variety efforts for management of stroke in this area to improve survival

\section{SS $18 / 4$ \\ Clinical Outcome of Metabolic Syndrome (MS). Epidemiological Data and Their Impact on Cerebral Stroke (CI)}

\author{
Bartko D. ${ }^{1}$, Combor I. ${ }^{2}$, Barakova A. ${ }^{3}$, Blazicek P. ${ }^{1}$, \\ Kuchtova Z. ${ }^{3}$, Pechanova O. ${ }^{4}$, Lietava J. ${ }^{5}$, Madarasz S. ${ }^{6}$, \\ Kovacik M. ${ }^{6}$, Rohalova J. ${ }^{6}$ \\ ${ }^{1}$ Central Military University Hospital, Institute of Medical \\ Sciences, Neurosciences, Military Health, Ruzomberok, \\ Slovakia, ${ }^{2}$ Central Military University Hospital, \\ Ruzomberok, Solomon Islands, ${ }^{3}$ National Center of Health \\ Informatics and Statistics, Bratislava, Slovakia, ${ }^{4}$ Slovak \\ Academy of Sciences, Institute of Normal and \\ Pathological Physiology, Bratislava, Slovakia, ${ }^{5}$ 2nd \\ Department of Internal Medicine, University, Bratislava, \\ Slovakia, ${ }^{6}$ Department of Neurology, Central Military \\ University Hospital, Ruzomberok, Slovakia
}

Background: MS is considered as very important RF of CI with high impact

Aim: 1. MS prevalence in Slovak population,

2. impact of individual MS components on CI risk.

Material and Methods: two groups for analysis: a) incidence of MS in Slovak population, b) incidence of MS in CI pts. Out of 6847 respondents (aged 15-64yrs), 5306 were identified for analysis.5 yrs follow-up.The IDF criteria (2005) were used.1600 pts with clear dg of CI. All five MS components were precisely characterized.

Results: Occurence of MS in population was $31.1 \%$ (31.0\%-men: 31.2\%-women). Gradual increase from 15-20 yrs $(4.0 \%: 4.3 \%)$ to $55-64$ yrs of age $(58.3 \%: 67.1 \%)$. Highest prevalence in arterial hypertension (AH), mainly with BMI $>37$. Similar trend in wais circumstances. Dominant RF in hospitalized pts (CVD/IHD) were non-treated AH and not-controlled AH. Pts with MS were 4-times more frequently hospialized comparing those without MS.Hospitalization time: 1121 days in MS compared to 436 days in those without MS. Every 9th man and 13th woman with combination CVD/IHD and MS were hospitalized comparing every 36th man and 50th woman without MS. High proportion of non-controlled $\mathrm{AH}$ in MS (38\%-men:48\%-women).In the group of CI pts significant changes in all components of MS were found in various degree of significance.Promised results showed analysis of new original LDL3-7 subfraction analysis

Conclusions: Growing MS prevalence in population and in $\mathrm{CI}$ pts with $\mathrm{MS}$ (mainly $\mathrm{AH}$, waist circumference, obesity) represent very important medical (mortality, morbidity, QOL), economical problem(hospitalization time, invalidization). ICVD/ IHD incidence rate are stagnating, not decreasing. This is main challenge to establish new national cerebrovascular and cardiovascular program

Supported by Governemental grants LPP0186-06, APVV0586-06, KEGA 5034-07, MS-008KU-8/2008 


\section{LB-SS/SS18/5 \\ Ischemic Stroke in 10 Years Old Child With High Titers of Antiphosphatidyl Serine Autoantibodies}

Ali H.M.

Technical \& Health Institute, Dean, Zakho- Kurdistan

Region, Iraq

Background: A 10 year old female child was presented with complete right sided hemiparesis. She was admitted in March 2003 to the Neurology Ward, Al-Raze Hospital, Mosul City, North Iraq. The condition developed suddenly 5 days prior to the admission.

Methods and Results: The parents denied any previous history of autoimmune diseases, bleeding tendencies, coagulation diathesis, and previous cerebrovascular attacks. All the performed haematological and routine serological profiles were normal. Magnetic resonance imaging (MRI) study revealed a lesion in the left basal ganglia. Testing for antiphospholipid autoantibodies (APLAs) was carried on coincidently on dual blood samples with 6 weeks apart and revealed high titer of $\operatorname{IgM}$ and $\mathrm{IgG}$ antiphosphatidyl serine and being negative for other tested APLAs which were lupus anticoagulant, anticardiolipin, and anti- $\beta 2$ glycoprotein I dependent. Consequently, an aspirin therapy was initiated by the physician and the child showed a gradual neurological improvement and discharged after 2 months.

Conclusion: This is the first case report of a childhood stroke case related to antiphospholipid syndrome and association with significant titers of the non-cardiolipin antiphospholipids, the anti-phosphatidyl serine, in Iraq and the region.

\section{LB-SS/SS18/6 \\ Pre-Hospital Delay After Stroke Onset: A Developing Nation Scenario}

\section{Chandra S. ${ }^{1}$, Agarwal D. ${ }^{2}$, Surana A. ${ }^{3}$, Khan M. ${ }^{3}$}

${ }^{1}$ All India Institute of Medical Sciences, Medicine, New Delhi, India, ${ }^{2}$ Mayo Clinic, Emergency Medicine, Rochester, USA, ${ }^{3}$ Mysore Medical College and Research Institute, Preventive and Social Medicine, Myosre, India

Introduction: Early treatment with thrombolytics has increased odds of improvement at 24 hours and favorable 3-month outcome compared to patients who get delayed treatment. Hence, we planned this study to emphasize on the delay in reaching to the hospital in patients experiencing stroke.

Methods: A cross-sectional study has been conducted over the period of 2 years; studying patients hospitalized for stroke in a tertiary health care center with major input from rural-population. Data was analysed using SPSS version 11.5.

Results: 134 patients (86-male, 48 -female, $55.22 \%$-smokers, $46.76 \%$-alcoholics) were studied, mean age was $53.83 \pm 18.02$ years [significantly lower in females (mean difference-9.73years $\mathrm{p}=0.002)]$. 40.30\% were known hypertensive and $14.18 \%$ were newly-diagnosed. Majority $(92 \%)$ of patients had locomo- tor and speech deficits, while $6 \%$ had pure speech deficit. Only $14.93 \%$ patients presented to hospital with in 5 hours of onset of stroke. This delay was $>24$ hours in $50 \%$ of female $29.06 \%$ of male patients. Over all, females have reached to the hospital later than males though this could not reach the statistical significance $(\mathrm{p}=0.095)$.

Conclusion: In this part of world, majority of the patients with stroke do not present immediately to the emergency department. This delay makes them less likely to be benefited by thrombolytic therapy.

\section{SS19}

Other Neurological Conditions/lssues

August 30, 2009

10:30-11:30

SS19/1

\section{Albanian Project on Epidemiology of Some Neurological Disorders}

\author{
Kruja J. ${ }^{1}$, Zerbi D. ${ }^{2}$, Beghi E. ${ }^{3}$, Kapisyzi M. ${ }^{1}$, Dobi D. ${ }^{1}$, \\ Kuqo A. ${ }^{1}$, Pupillo E. ${ }^{3}$, Millul A. ${ }^{3}$ \\ ${ }^{1}$ UHC Mother Theresa, Tirana, Albania, ${ }^{2}$ Fondazione \\ Pierfranco e Luisa Mariani per la neurologia infantile, \\ Neurologia Infantile, Milano, Italy, ${ }^{3}$ sstituto di Ricerche \\ Farmacologiche Mario Negri, Neuroscienze, Milano, Italy
}

Background: Epidemiological studies on neurological disorders in Albania are scanty and there are no comparative findings in the same population.

Methods: A door-to-door survey was undertaken in two separate geographic areas (Tirana and Saranda) with different sociocultural and economical background. Two random samples of the local population underwent a structured interview to ascertain headache, epilepsy, stroke, dementia, Parkinson's disease, multiple sclerosis, polyneuropathy and cerebral palsy. Each diagnosis was made using standard diagnostic criteria for epidemiological studies. For each case, the diagnosis was confirmed by neurological examination and, where available, the review of personal medical records. Overall lifetime prevalence ratios with $95 \%$ confidence intervals $(\mathrm{CI})$ were calculated by study area for each clinical condition.

Results: 9869 individuals were screened (Tirana 4953; Saranda 4916). The sample included 4867 men aged 1-91 years (mean 38.7) and 5002 women aged 1-96 years (mean 37.8). The overall crude prevalence ratios (per 1,000 population) were, in decreasing order: headache 257.8 (95\% CI 249.2-266.5), polyneuropathy 35.9 (95\% CI 32.2-39.5), epilepsy 15.2 (95\% CI 12.8-17.6), stroke 13.3 (95\% CI 11.0-15.5), dementia $11.0(95 \%$ CI 9.0-13.1), Parkinson's disease 8.5 (95\% CI 6.7-10.3), cerebral palsy 5.3 (95\% CI 3.8-6.7), and multiple sclerosis 0.3 (95\% CI 
0.1-0.9). Except for polyneuropathy (Tirana 43.8; Saranda 27.9), ratios were not significantly different in the two study areas.

Conclusions: The prevalence of some neurological disorders in Albania tends to differ from other European countries. Differences may be explained study design, structure of the target population and/or differing genetic and environmental factors.

\section{SS19/2 \\ Characteristics of Studies on Acupuncture for Neurological Disorders in Cochrane Reviews}

Yang W., Liu M., Wang D.

West China Hospital, Sichuan University, Department of Neurology, Chengdu, China

Objectives: To understand characteristics of studies on acupuncture for neurological disorders in Cochrane reviews.

Methods: We searched the Cochrane Library (CD-version Issue 1, 2009) for reviews on acupuncture in the treatment of neurological disorders. We analyzed characteristics of studies included in these Cochrane reviews.

Results: Seventeen Cochrane systematic reviews were included, of which four were on stroke and three on headache . 111 randomized controlled trials (RCT) were included in the thirteen completed reviews, of which 43 trials were conducted in China, 24 in Germany, 15 in UK, 9 in Sweden and so on. Of all the included $111 \mathrm{RCTs}, 47$ trials $(42.3 \%)$ used sham or placebo acupuncture as control interventions, and only 15 trials were with relatively higher quality regarding randomization, blinding, allocation concealment and fewer losses to follow-up. 7 of the 15 relatively higher quality trials were finished in Germany and five in UK, only one in China. Ninety-one trials reported data on follow-up. Primary outcomes used in the Cochrane reviews were reported by 65 trials, adverse events reported only in 11 trials. Two hundred and eighty-three trials were excluded from these reviews. Two reviews concluded that acupuncture could be effective while the remaining suggested that no firm conclusions can be drawn.

Conclusion: Acupuncture trials were conducted in many western countries as well as in China. Methodological quality of acupuncture trials needs further improvement. Acupuncture for stroke was assessed more frequently. Among 13 completed reviews only 2 reviews on headache got positive results and conclusions.

\section{SS19/3}

\section{The Neurobiology of Sexual Orientation}

Goldstein J.

San Francisco Clinical Research Center, San Francisco, USA

Homosexuality is a constantly debated issue as to whether it is determined at birth or a choice (nature vs. nurture). The works of the Kinsey Reports and Dr. Evelyn Hooker published in the 1950s resulted in the removal of homosexuality from the DSM4 in 1973. Since then, it has been mentioned as an illness only in the context of being a putative exacerbating factor in anxiety states. Recent studies reveal a clear cut neurobiology to sexual orientation.

Neurobiologist Simon LeVay conducted a study of brain tissue samples from 41 human autopsies performed at several hospitals in New York and California. He found a significant size difference of the interstitial nuclei of the anterior hypothalamus between homosexual and heterosexual men.

In addition, Dr. Ivanka Savic-Berglund and Dr. Per Lindström of the Karolinska Institute, Stockholm, performed fMRI and PET measurements of cerebral blood flow. Using volumetric studies, they found significant cerebral size differences between homosexual and heterosexual subjects; the brains of homosexual men resembled heterosexual women and homosexual women resembled heterosexual men. Pheromonal studies also have added to the scientific knowledge of sexuality. Sex-atypical connections were found among homosexual participants. Amygdala connectivity differences were found to be statistically significant and provided evidence towards sexual dimorphism between heterosexual and homosexual subjects. Extensive controls were performed during testing to exclude analytical variability.

A totally evidence-based medicine presentation will provide current data regarding homosexuality showing differences, or similarities, between the brains of homosexuals and heterosexuals.

\section{SS19/4 \\ A Systematic Review of Myasthenia Gravis Epidemiology}

Fitzpatrick A. ${ }^{1}$, Cardwell C. ${ }^{2}$, Mccarron P. ${ }^{2}$, Mcconville J. ${ }^{3}$
${ }^{1}$ Royal Victoria Hospital, Neurology, Belfast, United
Kingdom, ${ }^{2}$ Queens University, Epidemiology and Medical
Statistics, Belfast, United Kingdom, ${ }^{3}$ Ulster Hospital
Dundonald, Neurology, Belfast, United Kingdom

Background: Our aim was to examine myasthenia gravis (MG) epidemiology to see if there is worldwide consensus in incidence rate (IR), prevalence rate (PR) and mortality rate (MR) of the disorder and, if not, to investigate the influence of environmental and technical factors on these statistics.

Methods: 58 population-based studies were identified using multiple electronic databases, bibliography search and discussion with experts. Subgroup heterogeneity examination and regression 
analysis was used to assess the influence of study methodology, year and geographical area on observed rates.

Results: IR ranged from 1.7 to 21.3 per million person years and PR from 15 to 179 per million reflecting the massive heterogeneity across studies: $\mathrm{I}^{2}=96 \%$ (C.I.:95-98\%) and $\mathrm{I}^{2}=98 \%$ (C.I.:97$98 \%$ ) respectively. This heterogeneity remained within studies of similar methodology and geographical area and from studies performed within decades. On multivariate analysis, only year was significantly associated with IR and PR $(p=0.01, p=0.00)$ with a trend to increasing IR and PR with time $(r=0.57, r=0.66)$.

Conclusions: Case ascertainment and availability of diagnostic tests undoubtedly affect these observed frequencies and trends. However, the data also implies that environmental factors influence symptom onset resulting in the observed variation in IR across geographically and genetically similar populations

\section{SS19/5 \\ Late Onset Myasthenia Gravis - A Population Based Epidemiological Study in Northern Ireland}

\author{
Fitzpatrick A. ${ }^{1}$, Carwell C. ${ }^{2}$, Mccarron P. ${ }^{2}$, Mcconville J. ${ }^{3}$ \\ ${ }^{1}$ Royal Victoria Hospital, Neurology, Belfast, United \\ Kingdom, ${ }^{2}$ Queens University, Epidemiology and Medical \\ Statistics, Belfast, United Kingdom, 3Ulster Hospital \\ Dundonald, Neurology, Belfast, United Kingdom
}

Background: Over the past 10 years a number of studies from across Europe and Asia have shown evidence of an increasing incidence of myasthenia gravis (MG) in the elderly. It is not clear, however, if the observed increase is due to improved ascertainment or an actual increase in the frequency of the condition.

Methods: From 1990-2009, we performed an epidemiological study of MG in Northern Ireland. Dividing the study into 5 -year periods, we examined incidence rate, age-sex structure, ethnicity, use of diagnostic tests, diagnostic delay and who made the diagnosis. Age of onset above 50 years defined late-onset MG (LOMG) in this study.

Results: 195 new cases of MG were diagnosed over the 20 -year study period. The incidence rate of (early-onset) EOMG remained constant; while that of LOMG rose steadily from 4.8 (C.I.:0.7, 9.0) to 40.1 (C.I.:31.3, 48.9) per million. There was no difference between mean age at onset, sex structure, ethnicity, diagnostic delay or use of diagnostic tests across the time periods. No difference was observed in the proportion of cases diagnosed by neurology, ophthalmology, general practitioners or other medical specialities.

Conclusion: Our data suggests that diagnostic and referral patterns have not changed significantly over this time period, either have the age-sex or ethnic structure of this population. The steady EOMG incidence rate refutes a marked change in ascertainment methods with time. Therefore we propose an, as yet unidentified, environmental cause for the increasing frequency of LOMG.

\section{LB-SS/SS19/6 \\ A Computational Modelling Approach for the Discovery of Gene Interaction Related to Normal and Impaired Learning and Memory During LTP}

\author{
Kasabov N. ${ }^{1}$, Benuskova L. ${ }^{2}$, Schliebs S. ${ }^{1}$, Platel M. ${ }^{3}$ \\ ${ }^{1}$ Auckland University of Technology, KEDRI, Auckland, \\ New Zealand, ${ }^{2}$ University of Otago, Dunedin, New \\ Zealand, ${ }^{3}$ Rothamsted Research, London, United Kingdom
}

To study dynamic genetic mechanisms of learning and memory $[1,2]$ we have analysed mouse DNA temporal microarray data of 79 selected genes out of 12,488 activity regulated genes [3].

The 79 genes were selected based on their demonstrated relation to long-term potentiation (LTP), to normal learning and memory processes, and to brain diseases related to impaired learning and memory functions, namely: Alzheimer's disease, fronto-temporal dementia, X-linked mental retardation, Rett syndrome, and schizophrenia.

First, the gene expression time series of the 79 genes were clustered into 14 distinct clusters. Using an evolutionary optimization algorithm we have inferred a computational model that represents an abstract gene regulatory network between the 14 temporal clusters of genes.

The GRN model reveals new patterns of interaction between the selected genes during LTP. The model can be used for 'insilico' experiments to predict the effect of deletion (suppression) or insertion (activation) of genes involved in LTP. This was illustrated and confirmed with data from independent transgenic and gene knock-in mice experiments.

\section{References}

1. Benuskova, L., and Kasabov, N. (2007a). Modeling L-LTP based on changes in concentration of pCREB transcription factor. Neurocomputing 70(10-12), 2035-2040.

2. Benuskova, L., and Kasabov, N. (2007b). Computational Neurogenetic Modeling (New York: Springer).

3. Park, C. S., Gong, R., and Tang, S.-J. (2006). Molecular network and chromosomal clustering of genes involved in synaptic plasticity in the hippocampus. J. Biol. Chem. 281(40), 30195-30211. 


\section{Poster Presentations}

Neuro

epidemiology

P2

\section{Epidemiology of CNS Tumours and Infections}

P2/1

\section{Epidemiological Review of Neurosurgical Interventions of Insular Neoplasms}

Hussein K. ${ }^{1}$, Pasedag T. ${ }^{2}$, Brandis A. ${ }^{1}$, Kreipe H. ${ }^{1}$, Hussein $S^{3}$

${ }^{1}$ Medizinische Hochschule Hannover, Instiute of Pathology, Hannover, Germany, ${ }^{2}$ Department of Neurology, Klinikum Nordstadt, Hannover, Hannover, Germany, ${ }^{3}$ Department of Neurosurgery (formerly Department of Neurosurgery, Medizinische Hochschule Hannover)، Klinikum Hildesheim, Hildesheim, Germany

Background: The neurosurgical resection of insular neoplams is challenging, mainly due to the central localisation, the variable vascularisation and the complex neurosurgical anatomy. Therefore only few publications on histological findings are edited.

Methods: In order to improve the knowledge on this challenging field of neuroepidemiology/neurosurgery we performed a review of articles which report on the surgery and morphological subtypes of insular and para-insular neoplasms $(n=289$ patients, 301 operations). Furthermore, we summarise the clinical data and pathological findings of an own series of patients $(n=24$ patients, 37 operations).

Results: The summary of all available studies (including our series) on insular tumours revealed that astrocytoma WHO grade II $(21 \%, \mathrm{n}=72)$, glioblastoma grade IV $(17 \%, \mathrm{n}=57)$, astrocytoma grade III $(15 \%, \mathrm{n}=52)$ and oligoastrocytoma grade II $(12 \%$, $\mathrm{n}=42$ ) were the most frequent entities found in operation specimens $(\mathrm{n}=338)$.

In our cohort of patients, the frequency of higher tumour-grades (malignisation) was $21 \%(5 / 24$ patients) during follow-up while a previous study reported a frequency of $13 \%$ (3/23 patients).

Conclusion: As indicated by Duffau et Capelle (2004), this analysis shows that i) astrocytoma WHO grade II is the most frequent entity in a large number of insular tumours and ii) the frequency of the insular manifestation $(21 \%)$ exeeds that of the general frequency of cerebral astrocytomas $(8.5 \%$, according to CBTRUS, 2007).

\section{$\mathrm{P} 2 / 2$}

\section{Opportunistic Microbiological Agents in Glioma Patients}

\author{
Gousias K. ${ }^{1}$, Artzoglou V. ${ }^{2}$, Markou M. ${ }^{3}$, Voulgaris S. , \\ Polyzoidis K. ${ }^{2}$, Alamanos $Y^{5}$, Kyritsis A. ${ }^{6}$ \\ ${ }^{1}$ University Hospital, Department of Neurosurgery, \\ loannina, Greece, and University Hospital, Department of \\ Neurosurgery, Bonn, Germany, University Hospital, \\ Department of Neurosurgery, Thessaloniki, Greece, \\ ${ }^{3}$ Godeshoehe Klinik, Neurology, Bonn, Germany, \\ ${ }^{4}$ University Hospital, Department of Neurosurgery, \\ loannina, Greece, ${ }^{5}$ Universtiy of Patras, Public Health, \\ Patras, Greece, ${ }^{6}$ University Hospital, Department of \\ Neurology, loannina, Greece
}

Background: The role of human herpesviruses and toxoplasma gondii in the pathogenesis of brain malignancies remains obscure. The aim of our study was to investigate the association between CMV, EBV and toxoplasma gondii infection and the risk of glioma.

Methods: For CMV and EBV, a case-control study was carried out in 41 patients referred to the Department of Neurosurgery of the University Hospital of Ioannina, Greece, with newly histologically confirmed glioma and 41 age and gender matched controls. Concerning the toxoplasma gondii investigation, there were 23 cases and 23 controls. IgM and IgG for CMV, EBV and toxoplasma were determined in serum specimens using ELISA assays. Proportion between cases and controls were compared using the McNemars test.

Results: The glioma group showed the following positivity for IgG (CMV 39/41, EBV 36/41, toxoplasma gondii 11/23) and for IgM (CMV 5/41, EBV 4/41, toxoplasma gondii 0/23) while the control group showed for IgG (CMV 37/41, EBV 35/41, toxoplasma gondii 10/23) and for IgM (CMV 2/41, EBV 2/41, toxoplasma gondii $0 / 23$ ). Patients with glioma were more likely to have positive IgM for CMV and EBV compared to controls. However, the differences observed between cases and controls were not statistically significant.

Conclusions: Our findings revealed a trend but no statistically significant increased presence of IgM for CMV and EBV in glioma patients. However, this could be related to the limited number of patients included in the study. Further studies are needed to investigate the role of CMV and EBV in the pathogenesis of glioma.

\section{KARGER}

Fax +41613061234

E-Mail karger@karger.ch

www.karger.com
(C) 2009 S. Karger AG, Basel

Accessible online at: www.karger.com/ned 


\section{P2/3}

\section{Prevalence of Neurological Disorders in Patients with Pulmonary Tuberculosis}

\author{
Ivashynka A., Likhachev S.
}

National Neurology and Neurosurgery Research Center, Minsk, Belarus

Aim: to study the prevalence of neurological disorders (ND) in different categories of patients having tuberculosis of respiratory organs; to reveal polyneuropathy development risk groups.

Materials: 159 cases of new-onset patients and 99 patients with recurrent tuberculosis, who were treated in the Research Institute of Pulmonology and Phthisiology in 2006-2008, were analyzed. Indexes of 124 patients without neurological disorders and 134 patients having the disorders were compared.

Results: the prevalence of ND varied from $20 \%$ in new-onset patients to $50 \%$ in patients with recurrent and chronic forms of tuberculosis. The occurrence positively grew with the patients' age: from $32.1 \%$ in patients aged $18-35$ to $54.2 \%$ in patients aged 36-60, and 91.4\% in patients over $60(\mathrm{p}<0.001)$, and in patients with concurrent pathologies. All the patients with ND were experienced smokers. While the difference in the clinical forms of tuberculosis was not found, the new-onset patients with ND had more complaints (38.0\% and 54.2\% correspondently, $\mathrm{p}<$ $0.05)$, intoxication syndrome $(15.0 \%$ and $32.2 \%, \mathrm{p}<0.05)$, anemia ( 0 and $11.4 \%, \mathrm{p}<0.001)$, than in patients without ND. It was revealed that patients with ND had heavier clinical course and cases of recurrent tuberculosis. The analysis of chemotherapy tolerance demonstrated that the indexes of patients with ND on the main groups of side-effects were positively worse: $31.8 \%$ against $17.0 \%$ in patients without ND $(\mathrm{p}<0.001)$.

Conclusions: patients with recurrent tuberculosis, chronic pulmonary tuberculosis, over 60 , smokers, with pronounced clinical presentations and signs of tubercular intoxication, with heavy concurrent pathology (ischaemia, diabetes, alcoholism)

\section{P3}

Epidemiology of Dementia and Other Cognitive Disorders

\author{
P3/4 \\ Vascular Risk Factors and Cognitive Decline \\ - Results from the Invade Study \\ Broenner M. ${ }^{1}$, Bickel H. ${ }^{1}$, Etgen T. ${ }^{2}$, Sander D. ${ }^{3}$, \\ Briesenick C. ${ }^{4}$, Foerstl $\mathrm{H}^{5}$ \\ ${ }^{1}$ Technical University of Munich, Psychiatry and \\ Psychotherapy, Munich, Germany, ${ }^{2}$ Klinikum Traunstein, \\ Neurology, Traunstein, Germany, ${ }^{3}$ Medical Park Loipl, \\ Neurology, Bischofswiesen, Germany, ${ }^{4}$ The Invade Study \\ Group, Baldham, Germany, ${ }^{5}$ Technical University of \\ Munich, Klinikum rechts der Isar, Psychiatry and \\ Psychotherapy, Munich, Germany
}

Background: To analyse the association between vascular risk factors and cognitive decline.

Methods: Data are from the INVADE project (INtervention project of cerebroVAscular disease and Dementia in the district of Ebersberg), a prospective, population-based study. The crosssectional association between vascular risk factors and disease, assessed by General Practitioners and the participants themselves, and cognitive decline, measured with the 6 Item Cognitive Impairment Test (6-CIT), was analysed by means of multiple regression analysis, adjusting for potential confounders.

Results: A total of 3909 patients attended the project (mean age 67.7 years, $\mathrm{SD} \pm 7.8$, age range $55-102$ years; $59 \%$ female). A complete 6-CIT score is available for 3907 participants. Cognitive decline as indicated by a 6 -CIT score $>7$ occurred in $11 \%$ $(n=430)$ of the participants.

After adjustment for potential confounders in multivariate logistic regression analyses, age $(\mathrm{OR}=1.06$; CI: 1.04-1.07; $\mathrm{p} \leq 0.001)$, physical inactivity $(\mathrm{OR}=1.89 ; \mathrm{CI}: 1.41-2.54 ; \mathrm{p} \leq 0.001)$, depressive symptoms (GDS score) $(\mathrm{OR}=2.27$; CI: 1.71-3.01; $\mathrm{p} \leq 0.001)$, elevated homocysteine levels $(\mathrm{OR}=1.63$; CI: 1.17-2.27; $\mathrm{p}=0.004)$, elevated fasting serum glucose levels $(\mathrm{OR}=1.45 ; \mathrm{CI}$ : 1.07-1.98; $\mathrm{p}=0.017)$, elevated HbA1c levels (OR = 1.76; CI: $1.26-2.46 ; \mathrm{p} \leq 0.001)$ and elevated total serum cholesterol levels $(\mathrm{OR}=1.41$; CI: $1.11-1.78 ; \mathrm{p}=0.005)$ were independently associated with the prevalence of cognitive decline.

Conclusions: Several vascular risk factors seem to be independently associated with cognitive decline. These results are in accordance with other studies. 


\section{P3/5 \\ Vascular Risk Factors and Dementia: A 6-Year Follow-Up Study in General Practice}

\author{
Bickel H. ${ }^{1}$, Sander D. ${ }^{2}$, Broenner M. ${ }^{1}$, Foerstl H. ${ }^{1}$, for the \\ INVADE Study Group \\ ${ }^{1}$ Klinikum rechts der Isar, Department of Psychiatry and \\ Psychotherapy, Munich, Germany, ${ }^{2}$ Klinikum rechts der \\ Isar, Department of Neurology, Munich, Germany
}

Aims: To examine the association between vascular risk factors and incident dementia.

Methods: Data are from the INVADE project, a prospective study of general practice patients aged 55 years and over from a defined area in Upper Bavaria, Germany, who were members of the health insurance company with the largest market share. Risk factors were determined by patients' self-reports, information from the general practitioners, laboratory examinations, and ultrasound imaging. Information on incident dementia was obtained from the diagnostic records of the health insurance company. The relationship between risk factors and dementia during a six-year period was analysed by means of Cox's proportional hazard regression.

Results: Data were available for 3,813 participants who according to their GP did not suffer from dementia at the outset (mean age 67.0 yrs., $40 \%$ male). During follow-up dementia was diagnosed in 222 subjects. In the multivariable model all-cause dementia was significantly associated with age $(\mathrm{HR}=1.14$ per year; 95\% CI: 1.12-1.16), mild cognitive impairment $(2.03 ; 1.48$ $2.63)$, subjective memory impairment with $(2.34 ; 1.41-3.87)$ and without worries $(1.72 ; 1.29-2.29)$, physical inactivity $(1.92 ; 1.43$ $2.56), \mathrm{HbA} 1 \mathrm{c}>7.0 \%(1.78 ; 1.20-2.66)$, plasma homocysteine level $>15.0 \mathrm{mmol} / \mathrm{L}(3.15 ; 1.97-5.05)$, history of alcohol abuse $(3.26 ; 1.84-5.78)$ and of stroke (1.88; 1.18-3.00). Hypertension, hypercholesterolemia, intima-media thickness of the carotid arteries, ankle-brachial index, obesity, and other preexisting diseases were not related to dementia risk in the fully adjusted model.

Conclusions: These results are in line with other studies which have found an independent association between a sedentary lifestyle, diabetes, stroke and dementia.

\section{P3/6}

\section{A Belgian Observational Study on the Diagnosis and Comprehensive Care of Patients with Dementia: The Cognos Study}

Mets T. ${ }^{1}$, De Deyn P.P. ${ }^{2}$, De Lepeleire J. ${ }^{3}$, Ivanoiu A. ${ }^{4}$, Pals ${ }^{5}{ }^{5}$, Vandewoude M. ${ }^{6}$, Ventura M. ${ }^{7}$, Albert $A .{ }^{8}$, Mignon A. ${ }^{9}$

${ }^{1}$ University Hospital Brussel, Gerontology and Geriatrics, Jette, Belgium, 2University of Antwerp, Ziekenhuisnetwerk Antwerpen, Neurology, Antwerp, Belgium, ${ }^{3}$ Catholic University Leuven, General Practice, Leuven, Belgium, ${ }^{4}$ Saint Luc Hospital, University of Louvain, Neurology, Brussels, Belgium, 5 St Maarten Hospital, Neurology, Duffel, Belgium, ${ }^{6}$ University of Antwerp,

Ziekenhuisnetwerk Antwerpen, Geriatrics, Antwerp, Belgium, ${ }^{7}$ CTR Brussels and CUB Hospital Erasme, Neurology, Brussels, Belgium, ${ }^{8}$ University of Liège, Medical Statistics, Liege, Belgium, ${ }^{9}$ Pfizer Primary Care, Medical, Brussel, Belgium

Background: The Belgian healthcare system requires that dementia diagnosis is confirmed by a specialist, e.g., neurologist, psychiatrist or geriatrician. The current study aimed at documenting the diagnostic, therapeutic and care management of patients diagnosed with Alzheimer's disease (AD) and at identifying factors affecting patient management.

Methods: Cognos is a prospective, multicentre, longitudinal observational study, approved by a university hospital ethics committee. Data collection was done at diagnosis and 6 months thereafter, including a patient's questionnaire at both visits. Classical statistical methods were used to describe the patient population and to compare baseline and 6-month data. Baseline visit data will be presented.

Results: 87 specialists ( 72 neurologists and 13 geriatricians) recorded data for 720 patients (aged 79; M/F: 37\%/63\%) and 414 questionnaires were collected. MMSE scores ranged from 15 to 20 in $29.4 \%$ of the patients and from 21 to 26 in $56.8 \%$. One third of the patients lived alone, $61.8 \%$ with family and $6 \%$ in a retirement home. Consultation was initiated by the caregiver in $42.3 \%$ of cases and in $41 \%$ by the General Practitioner (GP). In the geriatrician setting, patients had more co-morbidities and functional problems. In $85 \%$ of the cases, other healthcare professionals were involved in the care, mostly the GP $(65 \%)$. A cholinesterase inhibitor was started in $70 \%$ of patients. Institutionalization was advised in 9.3\%. Quality of life was perceived as average. Need of help was inversely related to the MMSE score.

Conclusions: Cognos allows identifying factors that influence the choice of care provided. 


\section{P3/7 \\ Role of BDNF Val66met Polymorphism on the Association Between Physical Activity and Incident Dementia}

Kim J.M., Kim S.W., Shin I.S., Yoon J.S.

Chonnam National University Hospital, Psychiatry, Gwangju, Korea, Republic of Korea

Background: Beneficial effects of increased physical activity on cognitive outcomes have repeatedly been found in epidemiological \& clinical studies. A role of brain-derived neurotrophic factor (BDNF) has been suggested in animal models, but not yet tested in human. This study investigated modification by BDNF val66met polymorphism of the association between physical activity and incident dementia, since the met allele is recognised to be associated with lower BDNF activity.

Methods: Of 625 elders without dementia at baseline, 518 $(83 \%)$ were followed over a 2.4 year period and were clinically assessed for incident dementia and Alzheimer's disease (AD). Self-reported physical activity was recorded on a 4-point scale. Genotypes for BDNF val66met and apolipoprotein E (APOE) e4 polymorphisms were ascertained. Covariates included age, sex, education, depression, and vascular risk factors.

Results: Significant associations between lower physical activity at baseline and incident dementia and AD were modified by BDNF val66met polymorphism. The strength of association increased incrementally with the number of met alleles, and was strongest in those with the met/met genotype.

Conclusions: This study supports a previously suggested neurobiological basis for the effects of physical activity on dementia involving the BDNF system.

\section{P3/8 \\ Mild Cognitive Impairment Is Not Associated with Increased Mortality in the NEDICES Cohort Study}

Villarejo A. ${ }^{1}$, Bermejo-Pareja F. ${ }^{2}$, Trincado R. $^{3}$, BenitoLeón J. ${ }^{4}$, Rodríguez C. ${ }^{2}$, Puertas-Martin V. ${ }^{3}$, Medrano M.J. ${ }^{5}$, Boix R. ${ }^{5}$, Vega S. $^{6}$

${ }^{1}$ Hospital Doce de Octubre, Neurology, Madrid, Spain, 'University Hospital '12 de Octubre', CIBERNED, Department of Neurology, Madrid, Spain, ${ }^{3}$ University Hospital '12 de Octubre', Department of Neurology, Madrid, Spain, 'University Hospital '12 de Octubre', CIBERNED, Madrid, Spain, ${ }^{5}$ Epidemiology National Centre, Institute Carlos III, Madrid, Spain, ${ }^{6}$ Espinar Health Center, Segovia, Spain

Objective: To evaluate the mortality, ten year after the baseline wave (1994), of participants suffering Mild Cognitive Impairment (MCI) in the Neurological Disorders in Central Spain (NEDICES) Cohort Study, a population-based cohort study in the elderly (65 years and more) with 5278 participants at baseline.
The mortality of this cohort has been evaluated by means of the National Death Registry of Spain (NDRS) at 31-12-2004.

Methods: At baseline, diagnosis of MCI was made psychometrically in subjects with an MMSE score 1.5 SD below than mean of the people not cognitively impaired and with functional activities preservation. Persons with dementia or questionable dementia were excluded from analysis. The mortality at ten years was obtained from 1-05-1994 to 31-12-2004. Data were analysed with Cox proportional hazards model after making adjustments for potential covariates (age, sex, educational level and an index of co-morbidity).

Results: Of the 4883 non-demented participants at baseline, the crude mortality at ten years was: 221 deaths in $590(37.5 \%)$ MCI subjects and 1301 in 4293 (30.3\%) subjects with normal cognition. When adjusted for socio-demographic variables and co-morbidity, MCI was not statistically associated with an increased risk of mortality $(\mathrm{HR}=1.11$; CI 95\%: 0.96-1.29). Using the Kaplan-Meier method, mean survival was 8.7 years (CI 95\%: 8.5-9) for MCI and 8,9 years (CI 95\%:8.8-9.0) for normal cognition.

Conclusions: MCI is a heterogeneous condition and it remains controversial if it is associated with increased mortality. In our Spanish cohort, psychometrically defined MCI, is not statistically associated with an increased risk of mortality.

\section{P3/9}

\section{Memory Impairment in a Simple Word-Recall Task Increases Mortality in Non-Demented Individuals: A Population Based Study}

\author{
Villarejo A. ${ }^{1}$, Bermejo-Pareja F. ${ }^{2}$, Trincado R. ${ }^{1}$, Olazarán J. ${ }^{3}$, \\ Puertas-Martín V. ${ }^{1}$, Rodríguez C. ${ }^{2}$, Benito-Leon J. ${ }^{2}$, Vega S. , \\ NED/CES \\ ${ }^{1}$ Hospital 12 de Octubre, Neurology, Madrid, Spain, \\ ${ }^{2}$ Hospital 12 de Octubre, CIBERNED, Neurology, Madrid, \\ Spain, ${ }^{3}$ Hospital Universitario Gregorio Marañón, \\ Neurology, Madrid, Spain, ${ }^{4}$ Espinar Health Center, \\ Segovia, Spain
}

Objectives: To evaluate whether memory impairment in a simple three-word recall task increases the risk of mortality in a population-based survey of central Spain.

Design: Population census-based study. Evaluation of mortality after 10.67 years.

Setting and Participants: 3778 non-demented individuals included in the NEDICES (Neurological Diseases in Central Spain) cohort study, aimed at detecting age-associated neurological diseases in people aged 65 and over, living in one rural and two urban communities in Central Spain.

Main outcome measures: Cox's proportional hazards regression models were used to evaluate the risk of mortality according to performance in the three-word memory task included in the Minimental State Examination (score 0-3), adjusting for potential covariates (sex, age, level of education and comorbidity).

Results: After adjusting for confounding covariates, mortality was $52 \%$ greater in persons with the worst memory score 
(0) vs. persons with the best score (3). Hazard ratios showed a tendency to an increase in mortality from the best to the worst memory score, which was statistically significant for the groups with none (HR 1.52; CI: 1.27-1.80) or one (HR: 1.24; CI: 1.041.48 ) word recall. Older age, male sex and comorbidity were also associated with mortality, but level of education was not.

Conclusion: Memory impairment in the three-word delayed free recall, a very simple task used by physicians worldwide, increases the risk of mortality at ten years in non-demented elderly.

$\mathrm{P} 3 / 10$

\section{A New Scale for Gait Assessment in Alzheimer Disease: Preliminary Data for Validation}

Martinez-Martin P. ${ }^{1}$, Osa-Ruiz E. ${ }^{2}$, Gonzalez-Hervias L. ${ }^{2}$, Mendez-Robles N. ${ }^{2}$, Prieto-Jurcynska C. ${ }^{3}$, Kurtis M. ${ }^{4}$

${ }^{1}$ Carlos III Institute of Health, Alzheimer Disease Research Unit, Madrid, Spain, ${ }^{2}$ Alzheimer Center Reina Sofia Foundation-CAM, Physiotherapy and Rehabilitation, Madrid, Spain, ${ }^{3}$ Alzheimer Center Reina Sofia FoundationISCIII, Alzheimer Disease Research Unit, Madrid, Spain, ${ }^{4}$ Hospital Ruber Internacional, Department of Neurology, Madrid, Spain

Background: Study aimed at assessing the psychometric attributes of the new Rating Scale for Gait Evaluation in Alzheimer disease (RSGE-AD).

Methods: AD patients at any Global Deterioration Scale (GDS) stage admitted to the Alzheimer Center Reina Sofia Foundation without deficiencies impeding gait were included. Demographic data were recorded and the following measures applied: GDS, modified MMSE (MEC), Barthel Index (BI), Tinetti scale (TS), Timed Up \& Go test (TUGT), 7m test (steps and seconds to walk 7m), Global Evaluation of Gait (GEG), assessment of apraxia (upper limbs) and axial mobility (Steiger and Berman, 1993), and the tested scale. The RSGE-AD is a 14-item scale, each item scoring from 0 (normal) to 3 (severe), evaluating Functional ability (items 1 to 4 ) and gait (items 5 to 14), which may be summed to furnish a total score.

Results: 107 patients, age (mean \pm SD) $83.2 \pm 4.9$ years, $83.2 \%$ females, GDS stages 2 to 7 (median 5); and MEC score, $10.7 \pm 8.0$ $(\max =35$ points) participated. Mean RSGE-AD score was 17.1 \pm 11.2 (range: 0-41). No floor or ceiling effects were observed. For both subscales Cronbach's alpha was 0.94. Factor analysis identified one factor (70\% of variance). Correlations between RSGE-AD and other scales were: BI, -0.81; TS, -0.95; TUGT, 0.80 ; steps multiplied by seconds, 0.75 ; GEG, 0.95 ; apraxia scale, -0.47 ; and axial scale, -0.73 . Correlation between RSGE-AD subscales was 0.95 . RSGE-AD scores discriminated significantly among GDS levels $(\mathrm{p}<0.0003)$ and correlated moderately with $\operatorname{MEC}\left(\mathrm{r}_{\mathrm{S}}=-0,43\right)$.

Conclusion: The RSGE-AD performed as a promising tool for evaluating gait disorders in AD.

\section{P3/11 \\ Predictors of Institutionalization in Dementia-Free Older Adults}

Luppa M. ${ }^{1}$, Luck T. ${ }^{1}$, Matschinger H. $^{2}$, König H.-H. ${ }^{3}$, Riedel-

Heller S.G. ${ }^{1}$

${ }^{1}$ Department of Psychiatry, University of Leipzig, Public Health Research Unit, Leipzig, Germany, ${ }^{2}$ Department of Psychiatry, University of Leipzig, Leipzig, Germany, ${ }^{3}$ Department of Psychiatry, University of Leipzig, Health Economics Research Unit, Leipzig, Germany

Background: In previous decades a substantial number of community-based studies mostly including dementia cases examined predictors of nursing home admission (NHA) among the elderly. However, no one study has analyzed predictors of NHA for individuals without developing dementia before NHA.

Methods: For a population-based sample of 1,024 dementiafree adults aged 75 years and older, socio-demographic, clinical, and psychometric parameters were requested every 1.5 years over six waves. Kaplan-Meier estimates were used to determine mean time to NHA. Cox proportional hazards regression was used to examine predictors of long-term institutionalization.

Results: Of the overall sample, 7.8 percent of the nondemented elderly $(n=59)$ were institutionalized during the study period. Characteristics associated with a shorter time to NHA were increased age, living alone, functional and cognitive impairment, major depression, stroke, myocardial infarction, a low number of specialist visits and paid home helper use.

Conclusions: Although factors leading to NHA among individuals not developing dementia before NHA are similar to those driving the entry in population-based studies including dementia cases, the effect of severe physical or psychiatric diseases and living alone on NHA is considerably increased for dementia-free individuals.

\section{P3/12 \\ Dementia in a Hmong Community in French Guiana}

Nubukpo P., Pribisynscki V., Clément J.-P., Preux P.-M.

EA3174 Faculté de Médecine, Limoges, France

Backgrounds: Dementia, accounting for a major part of public health spending in many developed societies is becoming an economic burden in developing countries or societies. The review of the literature allows the report of a weak prevalence of dementia in the So call developing countries in particular of tropical and equatorial areas, close to 1 to $5 \%$ contrasting with higher prevalence in the Western Countries ( 5 to $20 \%$ ).

The aim of this study was to estimate the prevalence of dementia within the Hmong, a community coming from Laos and which migrated in French Guiana.

Methods: It is a cross-sectional study. Twenty six elderly aged more than 65 years in a rural population of 905 inhabitants were interviewed using the Community Screening Interview in 
Dementia (CSID) questionnaire. It was carried out by a single investigator assisted by a translator pertaining to the entourage of the questioned subject.

Results: The frequency of the cognitive impairment in this population is $0.66 \%$. The prevalence of the Alzheimer type dementia is $0.55 \%$, and vascular type dementia, $0.77 \%$. The female sex, the cardiovascular factors, the low level of schooling, the psychological traumatisms could be the risk associated factors of cognitive decline in this community.

Conclusion: A genetic origin or the presence of an environmental toxic (food, agriculture, pollution), could be considered. The cognitive disorders are well tolerated by the family.

\section{$\mathrm{P} 3 / 13$ \\ Prevalence of Dementia in Zaria, Northwestern Nigeria: Relationship to Age, Gender, Education, and Laboratory Test}

Ogboi J.S. ${ }^{1}$, Adeleye A. ${ }^{2}$, Obiakor O.R. ${ }^{2}$, Sabitu K. ${ }^{1}$, Abdulwahab A. ${ }^{3}$

${ }^{1}$ Ahmadu Bello University, Community Medicine, Zaria, Nigeria, ${ }^{2}$ Ahmadu Bello University Teaching Hospital, Medicine, Zaria, Nigeria, ${ }^{3} \mathrm{~A} h m a d u$ Bello University, Human Physiology (Neurophysiology), Zaria, Nigeria

Background: Dementia has become a public health problem in Nigeria due to the increase in elderly populations and lifestyle modifications leading to loss of productive life and disability. The prevalence of dementia and its relationship in northern Nigeria are unknown.

Aim: To estimate the prevalence of dementia and its clinical subtypes among northerners aged 55years or older and to examine associations with age, gender, and laboratory test.

Methodology: A simple cross-sectional descriptive study was carried out in Medicine department, Ahmadu Bello university teaching hospital, Zaria, Nigeria. Of 147 people screened with a vernacular adaptation of the Mini-Mental State Examination (MMSE), all those scoring at or below the cut-off of 23 were further evaluated. Diagnostic laboratory test investigations were employed to analyze serum samples tested for HIV antibodies using two sequential rapid HIV tests and one ELISA confirmatory test kit as recommended by the national algorithm for rapid HIV testing.

Result: A total of 147 patients were screened with the vernacular adaptation of MMSE comprising of $82 \%$ male and 18 female with a mean age of $62.2 \pm 4.4$. The prevalence of dementia was $34.1 \%$. The condition was early in $24.5 \%$, middle in $68 \%$, and late in $7.5 \%$.Of the subjects with dementia, $32 \%$ had vascular dementia, $1.4 \%$ had AIDS-related dementia, and $0.7 \%$ had senile dementia. Age and low education was significantly associated with dementia.

Conclusion: The prevalence of dementia among study group was high and dementia is an important health problem of the elderly population with intellectual disability. Age and low education are strongly associated with dementia. Identification of risk factors points towards the possibility of prevention

\section{LB-P3/102 \\ Dementia Mortality in NEDICES Cohort. Dementia Intensity is More Predictive than Dementia Aetiology}

Hernández Ramos F.J. ${ }^{1}$, Ramirez Moreno J.M. ${ }^{1}$, Varona F.', Sanchez-Ferro A. ${ }^{3}$, Trincado R. ${ }^{3}$, Boix R. ${ }^{4}$, Vega S. ${ }^{5}$,

Benito León J. ${ }^{6}$, Bermejo-Pareja F. ${ }^{6}$

${ }^{1}$ University Hospital of Badajoz, Neurology, Badajoz, Spain, ${ }^{2}$ Monteprincipe Hospital, Internal Medicine, Madrid, Spain, ${ }^{3}$ University Hospital 12 de Octubre, Neurology, Madrid, Spain, ${ }^{4} E$ pidemiology National Centre. Institute Carlos III, Madrid, Spain, ${ }^{5}$ Espinar Health Center, Segovia, Spain, 'University Hospital '12 de Octubre', CIBERNED, Neurology, Madrid, Spain

Objective: To evaluate the main predictors of mortality in demented patients detected in the baseline wave (1994-5).

Background: The neurological disorders in Central Spain (NEDICES) cohort study is a population-based cohort study in the elderly (65 years and more) with 5278 screened participants at baseline. The mortality in this cohort has been evaluated by means of the National Death Registry of Spain (NDRS) until 31-12-2004.

Methods: The main clinical characteristics of the 306 cases of dementia detected in the first wave (1994-5) were evaluated as mortality predictors by means of several statistical analysis (mainly COX regression and Kaplan-Meier survival analysis).

Results: The dementia subtypes were: Alzheimer disease (AD), 206 cases; vascular and mixed dementias (VaD) 57; Parkinsonism and dementia 14; secondary 5; undetermined 24. The dementia intensity (DSM-III-R) was; mild (93 patients), moderate (101patients), severe (79 patients) and undefined in 33 participants. At ten years, 1810 participants were dead. Age, sex (to be men) and the intensity of dementia were predictors of mortality in Cox regression analysis at ten years. Moderate dementia (HR 1,49 ; CI 95\%: 1.05-2.13); severe dementia (HR 2,53; CI: $1,74-3,68)$ vs. mild dementia. The mean survival time of VaD cases; 5,2 years (CI 95\% 4.0-6.5) was greater than in AD cases 4.7 years (CI 95\% 4,2-5,3) but not statistically significant.

Conclusions: Age, sex and dementia intensity but not aetiology were clear predictors of mortality at ten years in the NEDICES cohort study. 


\section{LB-P3/106 \\ Saliva Levels of Abeta1-42 as Potential Biomarker of Alzheimer's Disease: A Pilot Study}

Carro E. ', Antequera D. ${ }^{1}$, Vargas T. ${ }^{1}$, Molina J.A. ${ }^{2}$,

Puertas V. ${ }^{3}$, Bermejo-Pareja F.

'University Hospital '12 de Octubre', and

Neurodegenerative Diseases Biomedical Research Center (CIBERNED), Neuroscience Laboratory, Research Center,

Madrid, Spain, 'University Hospital '12 de Octubre', and

Neurodegenerative Diseases Biomedical Research Center (CIBERNED), Neurology Service, Madrid, Spain,

${ }^{3}$ University Hospital '12 de Octubre', Neurology Service,

Madrid, Spain

Background: Alzheimer's disease (AD) is diagnosed on clinical grounds, after excluding other pathological causes. A noninvasive biochemical marker that would support the clinical diagnosis and distinguish AD from other causes of cognitive decline would be of great value in the evaluation of AD patients.

Methods: Salivary samples were obtained from subjects with AD, age-sex-matched controls and Parkinson's disease (PD) patients. We analyzed saliva levels of $b$ amyloid (Ab) using a highly sensitive ELISA kit.

Results: We found a significant increase $(\mathrm{p}<0.01)$ in saliva of $\mathrm{Ab}_{42}$ levels in $\mathrm{AD}$ patients $(\mathrm{n}=42 ; \mathrm{M}: 10.54 \pm 4.1-\mathrm{SE}-\mathrm{pg} / \mathrm{ml})$ versus healthy controls $(\mathrm{n}=38 ; 2.81 \pm 0.9 \mathrm{pg} / \mathrm{ml})$ and PD patients $(\mathrm{n}=38 ; 3.82 \pm 0.58 \mathrm{pg} / \mathrm{ml})$. Saliva $\mathrm{Ab}_{40}$ levels were unchanged within all studied samples.

Conclusions: Saliva $\mathrm{Ab}_{42}$ levels could be considered a potential peripheral marker of $\mathrm{AD}$ allowing for discrimination of other types of neurodegenerative disorders if this preliminary finding is confirmed in a larger study.

\section{LB-P3/107 \\ Long-Term Reliability from a Spanish Adaptation of the Foltein's Minimental State Examination. Data from the NEDICES Cohort}

Moreno T.' Tapias E. ${ }^{2}$, Villarejo A. ${ }^{1}$, Trincado R. ${ }^{1}$, GarcíaRamos R. ${ }^{3}$, Puertas V. ${ }^{1}$, Vega S. ${ }^{4}$, Benito-León J. ${ }^{\text {, }}$ Bermejo-Pareja F. ${ }^{1}$

${ }^{1}$ Department of Neurology University Hospital "12 de Octubre", Madrid, Spain, ${ }^{2}$ Comillas Health Center, Madrid, Spain, ${ }^{3}$ Department of Neurology, Clinic University Hospital San Carlos, Madrid, Spain, ${ }^{4}$ Espinar Health

Center, El Espinar (Segovia), Spain

Objective: To evaluate the three years reliability from a Spanish adaptation of the Foltein's MiniMental State (MMSE-37) in the NEDICES participants free of neurological disorders.

Background: The NEDICES Study is a population-based cohort study in the elderly (65 years and more) with 5, 278 screened participants at baseline (1994-5) reviewed in a second wave (1997-8). MMSE test-retest reliability is rarely studied in census based populations.

Methods: A long term (three years) correlation (intraclass analysis) between the MMSE-37 scores in the first and second waves were performed. Participants suffering from neurological disorders affecting cognitive performance such as dementia and mild cognitive impairment, Parkinson and stroke in any of the two waves were excluded from the statistical analysis.

Results: At baseline, 4,029 participants performed the MMSE-37. Of them, 2,901 had another MMSE-37 at the second wave, but only 1,771 were free of neurological disorders affecting cognitive performance. The intraclass correlation between the two measurements was about 0.8 (0.81 in 1,326 participants younger than 75 years and 0.79 in 445 older than 75 years)

Conclusions: The long term MMSE-37 reliability was high in the participants of this cohort. Cognitive performance is quite stable in the elderly free of neurological disorders affecting cognitive performance.

\section{P4 \\ Developmental Disorders of the Nervous System}

\section{$\mathrm{P} 4 / 14$ \\ Neuroepidemiological Analysis of Developmental Articulation Disorder}

\author{
Golubovic S. ${ }^{1}$, Colic G. ${ }^{2}$ \\ ${ }^{1}$ Faculty of Special Education and Rehabilitation, Belgrade, \\ Serbia, ${ }^{2}$ Faculty of Special Education and Rehabilitation, \\ University of Belgrade, Belgrade, Serbia
}

Introduction: A child has an articulation disorders when he or she produces sounds, syllables, or words incorrectly so that listeners do not understand what is being said or pay more attention to the way the words sound than to what they mean. Most errors fall into one of three categories-omissions, substitutions, or distortions.

Method: Sample was made of 1000 children, age of 3,5- 7,0 years, 493 boys and 507 girls. Children were axamined by Test of articulation and Test of lateralisation.

Results: Correct articulation had 585 children (58,5\%), while articulation disorder had 415 children (41,5\%), 46,0\% of boys, and $37 \%$ of girls. Using lateralization had: right handed 888 $(88,8 \%)$, left handed $88(8,8 \%)$, and ambidexter $24(2,4 \%)$ children. Gestual lateralization had: right handed $637(63,7 \%)$, left handed $235(23,5 \%)$, and ambidexter $128(12,8 \%)$. Visual lateralization had: right handed 629 (62,9\%), left handed 298 (29,8\%), and ambidexter $73(7,3 \%)$. Auditive lateralization had: right handed $625(62,5 \%)$, left handed $241(24,1 \%)$, and ambidexter $133(13,3 \%)$. Lateralization of lower limbs: right $737(73,7 \%)$, left $108(10,8 \%)$, and ambidexter $155(15,5 \%)$. 
Conclusion: A child's overall speech pattern will usually become more undersandable as he or she matures, but some children will need direct training to eliminate all articulation errors. The exact speech pattern of the individual child will determine the answer to this question.

\section{P4/15 \\ Genetic and Clinical Analysis in Patients with Mental Retardation with Epilepsy or Developmental Delay}

\author{
Nakagawa E. ', Goto Y.-I. ${ }^{2}$, Japan Mental Retardation Resea \\ rch Consortium \\ 'Department of Child Neurology, National Center Hospital \\ of Neurology and Psychiatry, National Center of Neurology \\ and Psychiatry (NCNP), Kodaira, Japan, ${ }^{2}$ Department of \\ Mental Retardation and Birth Defect Research, National \\ Institute of Neuroscience, NCNP, Kodaira, Japan
}

Purpose: Recent studies have identified a series of genes involved in mental retardation (MR), but the current understanding of the genetic factors associated with MR is far from complete. Japan Mental Retardation Research Consortium has organized a research system at NCNP for genetic analysis of mental retardation. We evaluated the genetic and clinical features on MR accompanied with epilepsy or developmental delay.

Method: We have already established the research resource facility and collected samples under informed consent by providing the diagnostic service for known genetic defects or chromosomal abnormalities, such as genes for fragile $\mathrm{X}, U B E 3, A T R X$, MeCP2, FMR1, ARX, PQBP1, RPS6KA3, IL1RAPL1, TM4SF2, OPHN1, PAK3, FACL4, AGTR2, ARHGEF6, GDI1, SLC6A8, FTSJ1, ZNF41, DLG3, and copy number aberrations in X-tiling array CGH or high density oligonucleotide array analysis.

Results: As of the end of April in 2009, 260 pedigrees consisted of 125 families and 135 sporadic cases have been registered. We found $52 \%$ cases accompanied with epilepsy and $25 \%$ cases with developmental delay. We identified 35 positive genetic results for the examined about 150 cases by analyses of 19 $\mathrm{X}$-linked genes and array $\mathrm{CGH}$, which suggests in approximately $15 \%$ of unexplained mental retardation candidate causal genes were detected. In genetic positive cases, we found 10 cases with epilepsy and 10 cases with developmental delay.

Conclusion: By analyses of 19 X-linked genes and array $\mathrm{CGH}$ for unexplained mental retardation, in approximately $15 \%$ of patients possible disease-related genes were detected. In registered cases, $52 \%$ accompanied with epilepsy and $25 \%$ with developmental delay.

\section{P4/16 \\ Neuroepidemiological Analysis of Developmental Dysgraphy}

\section{Golubovic S.}

Faculty of Special Education and Rehabilitation, University of Belgrade, Belgrade, Serbia

Introduction: Dysgraphia is a disorder which expresses itself primarily during writing or typing, although in some cases it may also affect eye-hand coordination in such direction or sequence oriented processes as tying knots or carrying out a repetitive task. Impaired written language ability may include impairments in handwriting, spelling, organization of ideas, and composition.

Method: It was examined 1.000 children, age of 8 to 10 years. In some children it was examined writing and lateralisation: used, gestual, visual, auditive and lateralisation of lower limbs. For examining dysgraphy, The Scale for the Assessment of the Handwriting Dysgraphy (Ajuriaguerra \& Ozias, 1971. according to Cordic, A., \& Bojanin, S.1992) consists of taking handwriting samples in the three forms of writing: dictation, a free composition/spontaneous writing/description of an experience and rewriting. The texts of the dictation and rewriting were identical and contained all the 30 letters of the Cyrillic alphabet.

Results: Results show that fluency of appearance of dysgraphy in total sample is $25 \%$, and in boys $35 \%$, while in girls is 18 $\%$, Right used lateralisation have $88,8 \%$ children, lefthanded $8.8 \%$ and ambidexter 2,4\%. Right gestual lateralisation have $63,7 \%$, left $23,5 \%$ and ambidexter $12,8 \%$. Right visual lateralisation have $62,9 \%$, left $29,8 \%$, ambidexter $7,3 \%$. Auditive lateralisation to right have $73,7 \%$, left $10,8 \%$, ambidexter $15,5 \%$.

Conclusion: It is confirmed that fluency in appearance of dysgraphy in children of younger school age in this school is significantly bigger, in relation with frequency of dysgraphy which confirmed in earlier searches.

\section{P4/17 \\ Hemisphere Asymmetry by Charge Syndrome}

Kountouris D., Bougioukou A.

Neurological Diagnostic Center of Athens, Athens, Greece

Background: Charge syndrome is characterized by developmental disorder like ophthalmic coloboma, choanal atresia, hearing loss, ears malformations, small penis, facial palsy, dysphagia and patent ductus arteriosus.

In five years old boy with Charge syndrome we noticed a significant asymmetrical function of encephalic asymmetry.

Case Report: The child had an MRI brain examination that showed a characteristic encephalic asymmetry, and the smaller hemisphere to collateral side, where cited all the rest malformations, such as of the side of the ophthalmic colobomaa. The 24-hours EEG registration showed that the right (healthy) hemisphere had the more physiological functions (e.g. basic rhythmus) and the less pathological (e.g. epileptoform disorders). 
Conclusions: In Charge syndrome there is a dysfunctional EEG disorder only from the 'smaller' hemisphere.

\section{LB-P4/97 \\ Comparison of Neurodevelopmental Disability of Extremely Low Birth Weight Infants in Osaka for 2 Periods (1997-2001 and 2002-2006)

\author{
Shimamoto T. ${ }^{1}$, Fujimura M. ${ }^{2}$ \\ ${ }^{2}$ Osaka Medical Center and Research Institute for \\ Maternal and Child Health, Izumi City, Japan
} \\ ${ }^{1}$ Nara University, College of Liberal Arts, Nara City, Japan,}

Aims: The aim of this study is to survey mortality and neurodevelopment disability of extremely low birth weight infants in Osaka prefecture in 1997-2001 and 2002-2006, since health check systems for babies changed between these 2 periods. We also aim to evaluate the follow-up system between maternal and neonatal intensive care systems (operated in Osaka by 40 hospitals ` NICU) and public health centers.

Methods: Perinatal, neonatal and neurodevelopmental data at 18 months of 601 live-born ELBW infants born in 1997-2001, and 703 infants in 2002-2006 in Osaka, were analyzed according to the records from public health centers.

Results:

(1) Mortality rates of live-born ELBW infants in Osaka in 1997-2001 and 2002-2006 were $17.8 \%$ and $19.3 \%$ ( Total neonatal death rate).

(2) The rates of periventricular leukomalacia were $11.6 \%$ vs $10.5 \%$, cerebral palsy were $6.7 \%$ vs $5.3 \%$.

(3) The rates of severe neurodevelopmental impairment (major neurosensory abnormality with severe mental retardation) slightly decrease from $6.1 \%$ to $4.3 \%$, while the rates of slight mental retardation increased from $7.4 \%$ to $11.3 \%$.

(4) The rates of autism increased from $0.2 \%$ to $1.1 \%$.

(5) The percentages of missing cases increased from $10.5 \%$ to $23.8 \%$.

Conclusions: Cases with severe neurodevelopmental disability decrease while cass with slight mental retardation and autism increase, though further study is needed to check diagnostic methods used in different faculties. The follow-up system of ELBW infants between maternal and neonatal intensive care systems and public health centers in Osaka is now being revised to improve the results shown by this study.

\section{P5}

Epilepsy and Other Seizure Disorders Epidemiology

\section{P5/18 \\ Patterns of Emergency Admission to the Epilepsy Unit}

Gigineishvili $D$.

Department of Neurology and Neurosurgery, Javakhishvili State University, Tbilisi, Georgia

Background: Patients with seizure disorder present a challenge to specialists both in diagnostic categorization and in further management. This study investigated the pattern of seizure-related hospital admissions and its role in differential diagnosis of paroxysmal events.

Methods: All medical records of patients over age 10 admitted to the Epilepsy Unit at Sarajishvili Institute of Neurology \& Neurosurgery during one calendar year were reviewed. Data were compared using chi-square trend tests.

Results: 134 patients with clear discharge diagnosis were included in final analysis. Proven epileptic seizures were an admission reason in 100 cases, in remaining cases no active epilepsy was found. Female/male ratio was 3:7 and 10:7 accordingly $(\mathrm{p}=0.003)$. One-third of patients were admitted by emergency indications, i.e. within 24 hours after symptoms onset. But, emergency medical services [EMS] were used in $28 \%$ : only $25(25 \%)$ patients with epileptic seizures and 13 (38\%) with non-epileptic events were urgently transported to the hospital $(\mathrm{P}=0.139)$. Acute symptomatic seizures accounted for about $32 \%$ of patients with epileptic seizures delivered by EMS. Only 9 out of 100 patients with epileptic and 3 out of 34 with non-epileptic seizures were managed in an intensive care unit. True status epilepticus was observed in six cases.

Conclusions: Our results did not show significant differences of emergency admission pattern between those with definite epileptic and non-epileptic seizures. However, subgroup analysis identified high users of EMS among those with acute brain or mental illness. Further studies might shed light on the possible associations between these two categories of patients. 


\section{P5/19}

\section{Vagus Nerve Stimulation (VNS) in a Pediatric Population - Surgical Technique Considerations in Young Children}

Awaad Y.

King Fahad Medical City, Pediatric Neurology, Riyadh, Saudi Arabia

Vagus nerve stimulation (VNS) is beneficial for patients with intractable seizures (SZ's). Therefore, children are increasingly managed with VNS. However, there are specific technical surgical challenges in this population, which we evaluated in our own pediatric VNS implant series. Prospectively collected and reviewed detailed operative VNS implant reports (2002-2006) were correlated with out-patient follow-up evaluation, emphasizing the development of surgical technique adaptations in the small child as compared to older patients. Twenty-six children (age range: 14 months through 17 years; gender: 16 boys and 10 girls) with medically intractable SZ's (a VNS implant: 19 children were younger than twelve years. One six-year-old girl had a repeat VNS implant.

Results: All VNS electrode placements on the vagus nerve were performed using a transverse cervical incision which with increasing experience evolved into a key-hole type neurovascular exposure. The amount of anchor bulk and shape related skin irritation could be reduced by anchor customization. Pulse generator implant through an anterior axillary approach minimized incision visibility. All incisions remained cosmetically satisfactory. Three children required repeat surgeries: subcutaneous lead protrusion (7 and 5 years-old), pulse generator skin dehiscence (5 year-old), or generator dislodgement (14 years-old). One additional 5-yearold patient required implant removal because of infection. VNS implantation in small children poses additional challenges related to the relative implant bulk: wound complications are more common. Also, manipulative implant dislodgement is more common. Technique suggestions and pearls will be illustrated based upon our pediatric experience.

\section{$\mathrm{P} 5 / 20$}

\section{The Prevalence of Epilepsy in Korea}

Lee S.-Y. ${ }^{1}$, Jung K.-Y. ${ }^{2}$, Lee I.K. ${ }^{3}$, Yie S.-D. ${ }^{4}$,

Korean Epilepsy Society

${ }^{1}$ Kangwon National University, Neurology, Chuncheon-Si, Korea, Republic of Korea, ${ }^{2}$ Korea University, Seoul, Korea, Republic of Korea, ${ }^{3}$ Seoul Brain Neurology Clinic, Seoul, Korea, Republic of Korea, ${ }^{4}$ Keimyung University, Daegu-Si, Korea, Republic of Korea

Background: For Korean, there are no official epidemiologic data of epilepsy. We have an advantage to get data due to government-run medical security system that enrolls all citizens. We purpose to assess the prevalence of epilepsy in Korean population.
Methods: We used nationwide database from Korean National Health Insurance Cooperation. We defined epilepsy patients by diagnostic codes used for medical insurance claim in 2007. We calculated the prevalence of each age group, gender, and region.

Result: Overall prevalence was 3.1/1000. Age specific prevalence showed U-shape curve, lowest in 40s. In all age groups, the prevalence was higher in males than in females. There are more epilepsy patients in urban area than in rural area. The prevalence was highest, 5.2/1000 in Cheju Island.

Conclusions: The pattern of epilepsy prevalence in Korea is similar to that reported in recent studies. The prevalence is lower than that in studies performed before 1980s.

\section{P5/21 \\ Does Pre-Eclampsia Increase the Risk for Epilepsy in Adolescence and Early Adulthood? \\ Cossu P. ${ }^{1}$, Leoni S. ${ }^{2}$, Balata A. ${ }^{3}$, Riise T. ${ }^{1}$, Rosati G. ${ }^{2}$, \\ Department of Public Health and Primary Health Care, University of Bergen, Bergen, Norway, ${ }^{2}$ Department of Neuroscience and Mother and Child Care, University of Sassari, Sassari, Italy, ${ }^{3}$ Department of Neonatology, University Hospital of Sassari, Sassari, Italy}

Background: Preeclampsia has been found to be a risk factor for childhood epilepsy in the offspring, but whether the risk remains high even in adolescence and early adulthood is unclear.

Methods: We carried out a retrospective cohort study of 2557 live newborns between 01.01.1980 and 31.12.1981 at the Sassari University Dept. of Obstetrics and Gynaecology using medical charts compiled by neonatologists. Information on epileptic patients from the Epilepsy Case Register held at University Dept. of Clinical Neurology, the Sassari Health District discharge (ICD-9M 345) and prescription charge exemption records (017) was obtained. The cohort was followed from birth until either onset of epilepsy, death, emigration, or 31.12.2007. The association between preeclampsia and epilepsy was analysed calculating the incidence rate ratio ( $\mathrm{p}<0.05$ two-tailed test).

Results: We identified $69(2.7 \%)$ children who had been exposed to preeclampsia during their prenatal life. In 27 years of follow-up of the entire cohort 18 individuals received a diagnosis of epilepsy. Prenatal exposure to preeclampsia was associated with a 10.8-fold increased risk for epilepsy (CI 95\%: 2.381, 37.09; $\mathrm{p}<0.005)$. No significantly increased risk for epilepsy with onset before 12 years of age was found associated to pre-eclampsia.

Conclusions: This could suggest that either pre-eclampsia or its treatment may have an adverse effect on the developing brain and predispose to epileptogenesis, or that the association is due to confounders, such as preterm birth.

Acknowledgements: Regione Autonoma della Sardegna (Assessorato Igiene, Sanità e Assistenza Sociale, 2005); A Russo, PG Annicchiarico, I Spano, ML Turnu. 
P5/22

\section{Prospective Cohort Studies of Epilepsy - An Overview}

\section{Hylkema M. ${ }^{1}$, Leijten F. ${ }^{1}$, Wassenaar M. ${ }^{1}$, Uijl S. ${ }^{2}$ \\ ${ }^{1}$ University Medical Center Utrecht, Department Clinical \\ Neurophysiology \& Rudolf Magnus Institute of \\ Neuroscience, Utrecht, Netherlands, ${ }^{2}$ University Medical \\ Center Utrecht, Julius Center for Health Sciences and \\ Primary Care, Utrecht, Netherlands}

Background: A significant number of epilepsy patients remain refractory to medication, despite many studies on optimal treatment strategy. Research on management of epilepsy focuses on refractory patients actively seeking hospital care. A genetic basis of drug resistance has been proposed and can be investigated by a prospective cohort study which comprises a large number of patients with different responses to treatment. The objective of this review study is to give an overview of the main prospective cohorts of epilepsy patients over the last three decades. We want to see if these cohorts could be used to answer this question.

Methods: We searched for cohorts through a publication search with Pubmed. We used the terms: epilepsy, cohort, consecutive, follow up, follow-up, followed up, prospect*. Results were restricted to humans and publication date between January $1^{\text {st }}, 1980$ and June $15^{\text {th }}, 2008$. We excluded reviews, intervention studies, case reports, pilot studies, studies that included less than 200 persons, and journals with an impact factor below 1.5.

Results: This resulted in 79 publications, referring to 13 different cohorts. Remarkably, only one cohort was communitybased, whereas the others deal with patients in hospitals or specialised epilepsy clinics. Genetics was never the main objective.

Conclusions: We concluded that cohorts included a paucity of patients that responded well to treatment, probably because most cohorts were not population-wide. For a study into the genetic basis of medical intractability, a new cohort should be formed including patients treated by general practitioners or through pharmacy databases.

\section{P5/23}

\section{Characterization of Elderly Patients with Epilepsy in Two Hospitals in Bogotá, Colombia}

Velez van Meerbeke A. ', Gutiérrez-Álvarez A.M. ${ }^{1}$ Quintero Cusguen P. ${ }^{2}$, Palau D. 1, Ortiz Salas P. NeURos Research Group

${ }^{1}$ Universidad del Rosario, Research Department, Bogota, Colombia, ${ }^{2}$ Hospital de la Samaritana, Neurology Unit, Bogota, Colombia, ${ }^{3}$ Hospital Occidente de Kennedy, Neurology Unit, Bogota, Colombia

Background: Global prevalence of epilepsy in Colombia is $1.13 \%$. Despite the people is becoming older in the last decade and the group of 60 years and older are $10 \%$ of the general popu- lation, there's little information about this disease in this group of age.

Methods and Materials: A cross sectional, descriptive study in two hospitals in Bogotá, Colombia, among 2005-2008 was developed. Data bases and selected clinical records from epileptic patients 65 years and older were reviewed. Demographic data and information related to their condition and treatment was extracted.

Results: $179 / 211$ clinical records were reviewed and selected. The median age was 75 years (65-98) and the average of epilepsy onset was 67.5 years (7-93). In $64.4 \%$ the disease began after 65 years old. $84 \%$ of the seizures were classified as partial and $11 \%$ as generalized. The usual diagnosis was Focal Symptomatic Epilepsy (94.4\%). Etiology was reported as unknown in 58\% and cerebrovascular disease in $34 \%$. First generation antiepileptic drugs were most used (99\%) especially Phenytoin (68\%). 81/104 patients were not free of seizures.

Conclusions: De Novo epilepsy in the elderly is frequent. Inaccuracies in clinical registries, errors in classification, diagnosis and use of medication, lack of cognitive evaluation and adverse event reporting were found; these are major deficiencies in the treatment of epilepsy in the elderly. It's important to design clinical guidelines to answer the needs of these patients.

P5/24

\section{A Prevalence Investigation of Convulsive Epilepsy in Zayul County of Tibet Autonomous Region}

\author{
Yuhua Z. ${ }^{1}$, Dong Z. ${ }^{2}$, Long N. ${ }^{3}$, Yang C. ${ }^{4}$, Hong J. ${ }^{4}$, Mu L. ${ }^{4}$ \\ ${ }^{1}$ Neurologic Department, Sichuan University, Chengdu, \\ China, ${ }^{2}$ West China Hospital of Sichuan University, \\ Chengdu, China, ${ }^{3}$ Next Zayul Town Public Health Center, \\ Chayu County, TAR, Zayul, China, ${ }^{4}$ Next Zayul Town Public \\ Health Center, Zayul, China
}

Objective: To find out the prevalence rate of epilepsy, its treatment gap in Zayul county of Tibet Autonomous Region (TAR).

Methods: Cluster sampling was adopted to conduct a doorto-door epidemiological survey among 5903 rural population in ON and NEXT Chayu town located in Zayul county of TAR.

Results: One hundred -eighty epilepsy patients were diagnosed as convulsive epileptic patients, and the prevalence was $3.04 \%$. One hundred-five patients $(97.22 \%)$ were diagnosed as active epilepsy, with a prevalence rate of $2.96 \%$. The number of patients of cause of disease unclear are 145 (80.56\%); Ethyl alcohol related are 25 (13. 89\%): Skull flesh wound is $1(0.56 \%)$; Brain cysticercus is $1(0.56 \%)$, has the family history are $8(4$. $44 \%$ ). $99 \%$ patients diagnose the basis only to depend on the clinical outbreak. The activity epilepsy patients never had gotten treatment, treatment gap is $100 \%$. The locality non-medical personnel receive regular training which prevents and controls to epilepsy, the health agency have also not had one kind of conventional antiepileptic drug.

Conclusions: The prevalence rate and the percentage of untreated patients are higher than that previously reported in other 
regions, but there is still a $\mathrm{t}$ greater treatment gap. The patient has not accepted the treatment basically, it is urgent o provide local medical staff with training on treatment and prevention of epilepsy, and to heighten public health education.

$\mathrm{P5} / 25$

\section{A Study of the Significant of Changes of Neuropepide $Y$ and Neuron-Specific Enolase in Brain of Pentylenetrazol-Induced Rats}

\author{
Chen L. ${ }^{1}$, Chen Q. ${ }^{1}$, Ji X. ${ }^{2}$, Zou Z. ${ }^{2}$, Shen X. ${ }^{3}$, Lin L. ${ }^{3}$ \\ ${ }^{1}$ Fujian Provincial Hospital, Pediatrics, Fuzhou, China, \\ 2Fujian Provincial Governmental Hospital, Neurology, \\ Fuzhou, China, ${ }^{3}$ Fujian Provincial Key Laboratory For \\ Cardiovascular Disease, Fuzhou, China
}

Background: To evaluate the relationship between the NPY and epilepsy, and the effect of epileptogenesis upon brain damage.

Methods: 50male adult SD rats were randomly divided into experimental group and control group. The experimental group was injected IP with PTZ $(50 \mathrm{mg} / \mathrm{kg})$, and the control group $(\mathrm{A}=10)$ was injected IP with $\mathrm{n} / \mathrm{s}$. The treated group was divided into groups that seizured and a group that didn't seizure $(B=7)$ which were decapitated immediately. Groups of seizures were sacrificed at $T=0 \mathrm{~h}(\mathrm{C}=10), 6 \mathrm{~h}(\mathrm{D}=11)$ and $24 \mathrm{~h}(\mathrm{E}=10)$ after seizures. Blood samples were collected before sacrificed. EIA was used to evaluate the value of NPY and NSE in mesencephalon, hippocampus, striatum, frontal cortex and serum. The tissue expression of NPY and NSE in hippocampus was detected by immunocytochemical technics.

Results: The level of NPY in mesencephalon and frontal cortex of Group B is higher than that of Group A $(P<0.05)$. The level of NPY in the brain of seizure group is significant higher than that of control group. 24 hours after seizure, the level of NPY recovery to the values of non-seizure group. The level of NSE in serum, striatum and frontal cortex of Group B is higher than that of Group A $(P<0.05)$, it shows significant difference in hippocampus $(P<0.001)$. At 24 hours after seizure, the level of NSE in hippocampus and serum was still higher than that of control group $(P<0.01)$.

Conclusion: This experiment suggests that NPY may play an important role in epilepsy and seizure may cause the damage of brain tissue.

\section{P5/26
Brain Maldevelopment During Perinatal Period May Play an Important Role in Etiology of Cryptogenic Epilepsy}

Wang M., Wu L.

\section{Department of Neurology, Peking Union Medical College Hospital, Beijing, China}

It is widely accepted that idiopathic epilepsy is mainly affected by genetic factors. However, there are no good explains on cryptogenic epilepsy because any clues about history and imaging cannot be found. We hypothesized that the underlying damage caused during the perinatal period plays a key role in development of cryptogenic epilepsy but not idiopathic epilepsy. Data for this study was taken from the medical records of 2037 outpatients continuously visiting the epilepsy center of Beijing Union Hospital between 1/4/2007-1/4/2008. According to the 1989 proposal from ILAE, 468 cases of idiopathic epilepsy and 407 cases of cryptogenic epilepsy were included. The distribution of birth moth between these two groups was compared. The results showed that more cryptogenic epilepsy were born in December $(13.5 \% \mathrm{vs} 8.1 \%)$ and less were born in June $(5.9 \% \mathrm{vs} 9.8 \%)(\mathrm{Chi} 2=10.223, \mathrm{df}=2, \mathrm{p}=0.006)$ compared with idiopathic epilepsy. Within cryptogenic group, a December excess was also significant $\left(\mathrm{Chi}^{2}=24.735, \mathrm{df}=11, \mathrm{p}=0.010\right)$, while within idiopathic epilepsy, there was no significant difference in the frequency of birth month $\left(\mathrm{Chi}^{2}=2.513 \mathrm{df}=11, \mathrm{p}=0.996\right)$. The December excess and June absence in the patients with cryptogenic epilepsy will be a stronger support to the hypothesis that underlying damage caused during the perinatal stage may play a important role in development of cryptogenic epilepsy.

\section{P5/27 \\ Clinical Study on the Treatment of Acute Cerebral Infarction through Different Dosage Forms and Methods of Heparin}

Wang $Z$.

Hebei Medical University of China, Cangzhou City, China

Objective: To discuss the exact curative effective of heparin which were used to treat acute cerebral infarction, and different dosage forms, dosage and method were to discussed.

Methods: By stochastic, double-blind, the comparative clinical research, patients which conform to the standard are stochastically divided into 4 groups. After 3 weeks, the nerve functional scale, the case-fatality rate, the adverse terminal events were separately observed.

Results: There was significant difference in groups, each group were separately $94.46 \%, 86.69 \%, 85.02 \%$ and $76.33 \%(\mathrm{P}<$ 0. 01); Case fatality rates were separately $1.95 \%, 4.87 \%, 3.26 \%$ and $6.08 \%(\mathrm{P}<0.05)$, The difference had the statistics significance. The adverse terminal events of all the first 3 groups were higher than D group, but there were no statistics differences in the 
first 3 groups. Only 3 cerebral hemorrhage cases happened in the 615 examples.

Conclusion: Using the heparin through venous transfusion may remarkably reduce the case-fatality rate, increase cure and effective rate. Compared with LMWH and Blood-Activating Drugs it has even better effect. Furthermore, continued transfusion can achieve heparinized as soon as possible with the least amount and the best effect compared with injection once every day. Most important, the complications of hemorrhage didn't surpass the permission scope

P5/28

\section{Surveillance of Croatian Pregnant Women with Epilepsy and Effects of Antiepileptic Drugs Exposure in Their Offspring}

\author{
Miškov S. ${ }^{1}$, Gjergja Juraški R. ${ }^{2}$, Fučić A. ${ }^{3}$, Ivičević Bakuli T. ${ }^{4}$, \\ Cvitanović Šojat L. ${ }^{2}$, Vuković V. ${ }^{1}$, Basic Kes V. ${ }^{1}$, Demarin V. ${ }^{1}$ \\ ${ }^{1}$ Department of Neurology, University Hospital 'Sestre \\ milosrdnice', Zagreb, Croatia, 'Department of Pediatrics, \\ University Hospital 'Sestre milosrdnice', Zagreb, Croatia, \\ ${ }^{3}$ Institute for Medical Research and Occupational Health, \\ Zagreb, Croatia, ${ }^{4}$ Department of Obstetrics and \\ Gynecology, University Hospital 'Sestre milosrdnice', \\ Zagreb, Croatia
}

Introduction: The aim was to survey pregnancies exposed to AED and their offspring in order to assess teratogenic/neurodevelopmental effect of newer generation AEDs.

Methods: This is prospective surveillance of pregnancies in women with epilepsy (May2003-May2008). Pregnancy planning data, folic acid (FA) supplementation, seizure frequency and AED therapy were obtained.

Results: From 47 pregnancies: 83\% (39/47) exposed to monotherapy: 23 to lamotrigine (LTG): 13 live-births(LB), 2 premature deliveries, 2 spontaneous abortions (SA), 1 artificial abortion, 1 intrauterine death and 4 ongoing pregnancies(OP). Seven LB were exposed to carbamazepine (CBZ), 1 LB was under phenitoine (PHT) and 1 under phenobarbiton (PB) with EPH gestosis/ peripartal asphyxia. One preterm LB(ASD), severe psychomotor delay and epilepsy) was exposed to gabapentine (GBP), 3LB and $1 \mathrm{OP}$ were under valproic $\operatorname{acid}(\mathrm{VP})$. One LB and $1 \mathrm{SA}$ were under phenobarbiton (PB). Six pregnancies were exposed to polytherapy: topiramate(TPM)/VP(1 LB, 1 SA, 1 OP) CBZ/PB (1stillbirth); TPM/CBZ/PHT(1LB)with intrauterine growth retardation and dysmorphism); VP/clonazepam (CZP) (1 OP). Two women without AED therapy delivered healthy LB. From 35\% planned pregnancies, $20 \%$ took FA properly. About $25.5 \%$ of these women had their second or third pregnancy during our survey and their pregnancy planning was above $50 \%$ mainly due to preconceptional counseling.

Conclusion: Pregnancies under polytherapy resulted in larger proportion of complications. Besides 4 SA, 2 still-births, 2 premature deliveries, we have noted 1 possible intrauterine AED effect and 1 premature LB with ASD, psychomotor delay and epilepsy. Adequate preconceptional counseling in women with epilepsy resulted in higher pregnancy planning and FA intake. Follow up of LB till school age is needed.

\section{P6 \\ Migraine and Other Pain Disorders Epidemiology}

\section{P6/29 \\ Anticephalgic Photoprotective Premedicated Mask: A Report of a Successful Study of a Treatment for Migraine and/or Tension Headaches \\ Hyson $M$. \\ University of Nevada School of Medicine, Neurology, Las Vegas, USA}

Objectives: This study was performed to determine the efficacy of an anticephalgic photoprotective mask in conjunction with a topical medication containing bryonia and rhus toxicodendron in the treatment of migraine and/or tension headache.

Methods: Thirty-three patients were given masks and tubes of topical medication containing the bryonia and rhus toxicodendron. They were instructed to apply the medication to their frontalis and/or temporalis regions in the event they should suffer a headache and apply a photoprotective mask. Furthermore, they were instructed to take their usual oral or parenteral medications if required for the relief of the headache. They subsequently filled out forms rating the degree of relief which they attributed to the topical medication and the mask using a 0-10 scale. At the interview following the completion of their participation in the study, the patients were also simply asked if this form of treatment helped or not.

Results: Thirty out of 33 patients stated the medication and the mask were effective over and above the normal degree of relief they were receiving from their oral and/or parenteral medications. This study demonstrated a significant efficacy rate $(91 \%)$ in the treatment of migraine and/or tension headache with the anticephalgic mask in conjunction with a topical cream containing bryonia and rhus toxicodendron.

Conclusions: This study demonstrated a significant efficacy rate in the treatment of migraine and/or tension headache with the anticephalgic mask in conjunction with a topical cream containing bryonia and rhus toxicodendron. 


\section{P6/30}

\section{Prevalence of Migraine Headache in a Rural Area in Northern Tanzania: A Community- Based Door-to-Door Survey}

\begin{abstract}
Dent W. ${ }^{1}$, Stelzhammer B. ${ }^{2}$, Kerschbaumsteiner K. ${ }^{2}$, Meindl M. ${ }^{2}$, Kaaya J. ${ }^{3}$, Matuja W.B.P. ${ }^{4}$ Schmutzhard E. ${ }^{2}$, Winkler A.S. ${ }^{5}$

${ }^{1}$ Department of Neurosurgery, University Hospital of Zürich, Zurich, Switzerland, '2Department of Neurology, Medical University of Innsbruck, Innsbruck, Austria, ${ }^{3}$ Babati District Hospital, Manyara, Tanzania, United Republic of Tanzania, ${ }^{4}$ Department of Neurology, Muhimbili University of Health and Allied Sciences, Dar es Salaam, Tanzania, United Republic of Tanzania, ${ }^{5}$ LudwigMaximilians-University, Interdisciplinary Centre for Palliative Care and Department of Neurology, Munich, Germany
\end{abstract}

Background: In 2004, the initiative 'Lifting the burden: The global campaign against headache' was launched. One goal is to bring out all existing evidence about the burden of headache worldwide. In our paper, we shed more light on headache in subSaharan Africa (SSA) by reporting the prevalence of migraine headache in a rural population of northern Tanzania.

Methods: From December 2003 till June 2004 a communitybased door-to-door survey was carried out, using the criteria of the International Headache Society (IHS). 1,192 households with 7,412 individuals were included.

Results: We found an overall 1-year migraine prevalence of $4.3 \%(316 / 7,412 ; 95 \%$ CI $3.8,4.7)$ with an age-adjusted rate of $6.0 \%$ and a male:female ratio of $1: 2.9(p<0.001)$. One hundred eighty-four people met all criteria for migraine resulting in a 1-year prevalence of $2.5 \%(184 / 7,412 ; 95 \%$ CI $2.1,2.9)$ and a male:female ratio of $1: 2.5(\mathrm{p}<0.001)$. The remaining 132 individuals did not fulfill all criteria for migraine headache, hence, these people were classified as migrainous disorders resulting in a crude prevalence rate of $1.8 \%(132 / 7,412 ; 95 \%$ CI $1.5,2.1)$.

Conclusions: The present survey shows that migraine headache is not uncommon in northern Tanzania. The recorded migraine prevalence is located within the median of previous African prevalence surveys, which confirms the trend of lower migraine frequencies in rural Africa compared to western countries. Based on these findings, the burden of migraine may be calculated; data analysis currently is underway.

\section{P6/32 \\ Nociceptive Responses in Primary Somatosensory Cortex of the Rat}

Chen C.-P., Jaw F.-S.

Institute of Biomedical Engineering, Naional Taiwan

University, Taipei, Taiwan, Republic of China

Background: Nociception or pain in clinical is an important issue in past decades. It is known that the primary somatosensory cortex (SI) mainly contributes on discrimination in many sensations, however, nociception in this area is still unclear due to its complexity. In recent years, infrared laser pulses have gained widespread clinical and experimental use. However, laser pulse would induce heat and noxious sensation which would be transmitted through $\mathrm{A}_{-\mathrm{d}}$ and $\mathrm{C}$-fiber. In the present work, a pincher that has a synchronous output signal was used to collect pinch-evoked action potentials. A spike-sorting program (designed by our lab) was used to detect nociceptive specific (NS) units and to manifest the distribution of NS neurons within laminars of SI.

Methods: Wistar rat weighted 250 350 g were used. Experimental protocols were carried out ethically. Pinches were applied on the receptive (i.e. rat tail) field. Detection of action potentials was carried out by a program written in MatLab.

Results: The numbers of the NS units induced by pinch is more than control (without pinch). Their distribution among laminars in SI showed that the highest NS activity was in a depth of $800 \mu \mathrm{m}$, which corresponding to laminar IV.

Conclusion: The present work combined a quantified pinch stimulator with a synchronous trigger output to identify the NS units and a sorting algorithm to intuitively display comparison of the neuronal activities among laminars of the cortex. This preliminary result suggests that different laminars of the rat cortex may have engaged in handling different modalities of sensations.

P6/33

\section{Atorvastatin Attenuates NF-kappaB Activation in Trigeminal Nucleus Caudalis in a Rat Model of Migraine}

\author{
Yin Z., Fang Y., Wang X., Zhang A., Li X., Ren L. \\ Department of Neurology, the First Affiliated Hospital, Sun \\ Yat-Sen University, Guangzhou, China
}

Background: NO-induced NF-kappaB activation in trigeminal nucleus caudalis(TNC) is believed to be involved in the pathogenesis of migraine. Atorvastatin, an inhibitor of HMG-CoA reductase, is thought to has pleiotropic effects including neuroprotection as well as anti-inflammatory properties in addition to its original role in lowering cholesterol. Morever, there are several lines of evidences that atorvastatin inhibits NF-kappaB activation in blood mononuclear cells. We herein test the hypothesis that atorvastatin can also attenuate NF-kappaB activation in rat TNC in a NO-induced migraine model.

Methods: Male Sprague-Dawley rats (200-230 g) were divided into 3 groups as followings:(1) control group(no drug administration),(2) nitroglycerin (NTG) administration (10mg/ $\mathrm{kg}$, i.p.), (3)atorvastatin pre-treatment followed by NTG administrtion:rats were orally pre-treated with atorvastatin $(1,10$ $\mathrm{mg} / \mathrm{kg}$ respectively) 2 hours prior to the administration of NTG $(10 \mathrm{mg} / \mathrm{kg}$, i.p.). Four hours after injection of NTG, rats were transcardially perfused and the cervical part of the TNC was removed for NF-kappaB analysis using fluorescent immunohistochemistry and western blotting technique.

Results: A significant increase of nuclear immunostaining of p65, an indicator of NF-kappaB activation, was detected in TNC in rats following injection with NTG when compared with the 
control group $(\mathrm{p}<0.05)$. However,the NO-induced NF-kappaB activation in TNC was attenuated by pre-treatment with atorvastatin in a dose-dependent way $(\mathrm{p}<0.05)$.

Conclusions: .These results suggest that atorvastatin may be a novel and underlying candidate for treatment of migraine via attenuating activation of NF-kappaB in TNC .

P6/34

\section{Clinical Characteristics of Migraine and Probable Migraine in General Population}

\author{
Vuković V. ${ }^{1}$, Plavec D. ${ }^{2}$, Lovrenčić-Huzjan A. ${ }^{1}$, Budišić M. ${ }^{1}$, \\ Demarin $V^{1}$ \\ ${ }^{1}$ Department of Neurology, University Hospital 'Sestre \\ milosrdnice', Zagreb, Croatia, 'Department of Research, \\ Childrens Hospital 'Srebrnjak', Zagreb, Croatia
}

Background: Migraine is a frequent primary headache, but often under diagnosed. Probable migraine is even more a neglected condition due to lacking symptoms which masks the diagnosis. The aim of this study was to determine the clinical characteristics of strict migraine (SM) and probable migraine (PM) in general population.

Methods: The data were collected from a cross-sectional survey of an adult population sample. Randomly selected individuals ( $>18$ years of age) from the general population were asked to fulfill a self-completed questionnaire. The questionnaire was designed aiming to determine the clinical characteristics of the headache according to the ICHD-2 criteria for SM and PM.

Results: The study included 115 patients with SM and 174 patients with PM. Significant difference was observed in the presence of nausea (SM 53.0\% vs. PM 40.2\%, P =0.007), photophobia or phonophobia (SM $60.9 \%$ vs. $49.4 \%, \mathrm{P}=0.04$ ) and the aggravation by physical activity (SM $40.0 \%$ vs. PM $48.3 \%, \mathrm{P}=0.005$ ). There was no significant difference in reported unilateral headache (SM 62.6\% vs. PM 64.4\%), pulsating quality (SM 54.8\% vs. PM 58.1\%), intensity, duration of headache (SM 1.38 vs. PM 1.29 , mean number of days with headache), frequency (SM 2.03 vs. PM 2.08, mean number of headache attacks per month), relation to menstruation and the presence of aura, all $\mathrm{P}>0.05$.

Conclusions: Patients with strict migraine more frequently report nausea and photophobia or phonophobia while patients with probable migraine report aggravation of headache with physical activity. Bringing to attention clinical differences should help clinicians to diagnose each condition more accurately.

\section{$\mathrm{P} 6 / 35$}

\section{Prevalence of Chronic Headache in Croatia}

Vuković V. ${ }^{1}$, Lovrenčić-Huzjan A. ${ }^{1}$, Budišić M. ${ }^{1}$, Plavec D. ${ }^{2}$, Demarin $V^{1}$

${ }^{1}$ Department of Neurology, University Hospital 'Sestre milosrdnice', Zagreb, Croatia, 'Department of Research, Childrens Hospital 'Srebrnjak', Zagreb, Croatia

Background: Chronic headache describes the presence of migraine and/or tension-type headache on $\geq 15$ days per month on average for $>3$ months and fulfills the rest of the IHS criteria. The prevalence of chronic headache is within the range of $1.4-7.3$ $\%$ worldwide. The aim of this study was to determine the 1 -year prevalence of chronic headache in adult Croatian population.

Methods: The data were collected from a cross-sectional survey of an adult population sample. Randomly selected patients from the general population in four Croatian cities and their suburbs were asked to fulfill a self-completed questionnaire. The study population included adults $>18$ years of age. The prevalence of chronic and daily headache was calculated in the sample representing 3383769 Croatian adults.

Results: The total sample included 1542 responders among which 616 with headache. Among 616 responders, 4.9\% had chronic headache. The 1-year prevalence of chronic headache was $1.9 \%$. The prevalence of daily headache was $0.9 \%$. According to these results, 64291 adult inhabitants in Croatia suffer from chronic headache from which 30454 from daily headache.

Conclusions: The prevalence of chronic headache in Croatia is comparable to other countries worldwide. Translated into absolute numbers, a significant number of patients suffer from chronic or daily headache. These patients require special attention and should be offered medical support.

\section{$\mathrm{P} 6 / 36$ \\ Headache Management in a Neurological Emergency Room}

Vuković V. ${ }^{1}$, Knežević-Pavlić M. ${ }^{2}$, Tumpić-Jaković J. ${ }^{3}$, Strineka M. ${ }^{1}$, Lovrenčić-Huzjan A. ${ }^{1}$, Demarin V. ${ }^{1}$

${ }^{1}$ Department of Neurology, University Hospital 'Sestre milosrdnice', Zagreb, Croatia, '2Department of Neurology, General Hospital Našice, Našice, Croatia, ${ }^{3}$ Department of Neurology, General Hospital Virovitica, Virovitica, Croatia

Introduction: Headache is a frequent complaint among patients in the ER. The aim of this study was to analyse the management of headache patients presenting to the emergency room (ER) at the University Hospital in Zagreb.

Methods: A retrospective analysis of all patients with headache was carried out during 2007. Patients were analyzed according to the diagnoses, diagnostic procedures, treatment and further refferal.

Resuls: Among 6225 patients, 1385 (22.3\%) complained of headache; 894 (64.9\%) women and 491 (35.4\%) men. Migraine with or without aura, tension-type headache or cervicogenic head- 
ache had $1009(72.9 \%)$ of patients (67.3\% women and $32.7 \%$ men); 84 (6\%) had intracranial haemorrhage, 33 (2.3\%) had primary tumour, 54 (3.8\%) metastases, 193 (13.9\%) head trauma, 7 $(0.5 \%)$ head trauma with heamorrhage and $5(0.4 \%)$ had an infective disease. A diagnostic procedure was indicated in $413(29.8 \%)$ of patients: $314(22.7 \%)$ had a CT scan, 85 (6.1\%) an EEG and 70 $(5 \%)$ had an ultrasound examination. Patient refferal was as following: 1022 (73.8\%) was dismissed home, 222 (16\%) was reffered to other clinics and $141(10.2 \%)$ was hospitalized. Among patients with primary and cervicogenic headaches a diagnostic procedure was performed in $235(23.2 \%)$ while $45(4.5 \%)$ was hospitalized.

Conclusions: Patients with primary headaches visit the ER in a relatively high number which represents a huge economical burden for the health system. Better treatment for these patients should be provided by GPs and neurologists in out-patient headache clinics.

\section{P6/37}

\section{Analgesic Overuse is More Common in Depressive Migraine Patients}

\section{Vuković V., Mikula I., Lovrenčić-Huzjan A., Budišić M., Demarin $V$. \\ Department of Neurology, University Hospital 'Sestre milosrdnice', Zagreb, Croatia}

Introduction: The aim of our study was to determine the relationship of depression, number of headache days/month and the number of used analgesics and triptans in a group of patients attending the outpatient-headache clinic.

Methods: 66 patients (58 women, 8 men) were leading a headache diary during 3 months and fulfilled the Beck depression questionnaire which classifies the score 1-13 as minimal depression (group A), 14-19 mild (B), 20-28 moderate (C) and 29-63 significant (D).

Results: There was 30 patients in group A, 17 in B, 10 in $\mathrm{C}$ and 9 in $\mathrm{D}$; Beck depression score was 6,$8 ; 14.9 ; 22.8$ and 42.7 respectively. Migraine with or without aura had 54 patients, tension-type headache 6 and a combination headache 6 patients. Mean number of headache days/month in group A was $11.3(+/-$ 7.2), in B $11.1(+/-7.2)$, in C $16.9(+/-10.1)$ and in D $14.2(+/-$ 9.3); the number of days with headache was significantly higher in group $\mathrm{C} \mathrm{P}<0.05$. Mean number of taken tablets in group $\mathrm{A}$ was $24.5(+/-32)$, in B $25.5(+/-30)$, in C $42.4(+/-29)$ and in D 42.4 $(+/-22)$. Patients in groups $\mathrm{C}$ and $\mathrm{D}$ were taking significantly more medications, $\mathrm{P}<0.05$.

Conclusions: Patients with the highest Beck depression score are more likely to take more medications for headache attacks compared to patients with lower scores although they do not differ significantly in the number of days with headache. Our results confirm earlier reports that medication overuse headache is a part of the spectrum of addictive disorders.

\section{P7 \\ Motor Neuron Disease Epidemiology}

P7/38

Amyotrophic Lateral Sclerosis in the Area of
Alt Empordà (Northeast of Catalonia):
An Epidemiologic Study

Pablo M.J., Moreno E., Carmona O., Ciudad R., Güell E., Osuna T., Martínez R., Paz M.

Fundació Salut Empordà, Figueres, Spain

Background: Amyotrophic Lateral Sclerosis is a degenerative motorneuron disease which produces progressive generalized weakness, muscular atophy and fasciculations, leading patients to death in few years. We performed an epidemyological study of ALS in the Alt Empordà area, a well-defined region in the North East of Catalonia, with a population of 124000 inhabitants. There are only two ALS epidemyological studies in Spain.

Objectives:

- To obtain the prevalence of ALS in $15^{\text {th }}$ September 2006.

- To calculate a retrospective dianose incidence data between 2004 and 2008.

Methods: We present a retrospective study using the diagnosis register of the reference area hospital. Inclusion criteria were: Definite ALS by El Escorial criteria. Clinical, demographic and electromyographic data were recorded.

Results: 18 patients fulfilled the criteria for ALS. Male/ female ratio was $1: 1$. The mean age at onset was 60.5 years, 57.3 years in men and 63.6 years in women. The clinical form of onset were bulbar in $33 \%$ of the patients, $27 \%$ spinal-EESS, $16 \%$ spinal-EEII and generalized in $22 \%$. No sex realted differences were found in bulbar form. Only 1 patient with familial ALS was found (5.55\%). The mean time latency of diagnosis was 6.17 months, and the median survival was 22.5 months. The prevalence rate in $15^{\text {th }}$ September 2006 was $4.03 / 100000$ inhabitants. The incidence rate during the selected period was 1.61/100000 inhabitants.

Conclusions: Prevalence and incidence rates in the Alt Empordà area (Northeast Catalonia) are similar to the obteined in other studies. A prospective study of incidence has started in 2009 to confirm our data. 
P8

\section{Multiple Sclerosis and Other Demyelinative Disorders Epidemiology}

\section{P8/39 \\ Cancer Risk and Multiple Sclerosis: Preliminary Results from the Malignancy in Multiple Sclerosis [MAMS] Study

\author{
Kingwell E. ${ }^{1}$, Bajdik C. ${ }^{2}$, Phillips N. ${ }^{2}$, Oger J. ${ }^{1}$, \\ Hashimoto S. ${ }^{1}$, Tremlett $H^{1}{ }^{1}$ \\ ${ }^{1}$ University of British Columbia, Medicine (Neurology), \\ Vancouver, Canada, ${ }^{2}$ BC Cancer Agency, Vancouver, \\ Canada
}

Background: There is a lack of consensus regarding the risk of cancer in multiple sclerosis (MS). The objective of this study was to investigate the incidence of cancer in a cohort of MS patients compared to the general population in British Columbia (BC), Canada.

Methods: Definite or probable MS patients residing in $\mathrm{BC}$ who visited a BC MS clinic before Dec/04 were selected from the BCMS database (estimated to capture $80 \%$ of MS patients in $\mathrm{BC}$ ) and linked to records from the BC Cancer Agency database (estimated to capture $>99 \%$ of invasive cancers in BC from 1969). Incidence of malignancy following MS onset was compared to the age- and sex-matched incidence in the BC population by calendar year.

Results: 6820 MS patients (mean age at MS onset $=32.2$ years; $72 \%$ women) met selection criteria with 105,744 personyears of follow-up. Standardized incidence ratios (SIRs) for all cancers combined were 0.55 (95\% CI, 0.41-0.73) for men and 0.62 (95\% CI, 0.52-0.72) for women. Reduced risk was observed for breast (SIR $=0.79,95 \% \mathrm{CI}, 0.61-0.99)$ lung (SIR $=0.19 ; 95 \%$ CI, 0.08-0.38) and colorectal (SIR $=0.38 ; 95 \% \mathrm{CI}, 0.20-0.66)$ cancers. The risk of other cancers, including prostate and brain were no different to expected rates.

Conclusions: Cancer incidence rates are significantly lower in BC patients with MS compared with the general population. Further study will investigate the influence of exposure to immunomodulatory treatments for MS on these cancer rates. The mechanism(s) responsible for the reduced risk also warrant further investigation.

\section{P8/40}

\section{Geographical Associations of Select Environmental Factors with the Multiple Sclerosis Prevalence in Värmland, Sweden: An Ecological Study}

\author{
Lauer K. ${ }^{1}$, Callander M. ${ }^{2}$, Boström I. ${ }^{2}$, Landtblom A.-M. ${ }^{2}$ \\ ${ }^{1}$ Epidemiologist, Griesheim, Germany, ${ }^{2}$ University \\ Hospital, Division of Neurology, Linköping, Sweden
}

Background: In epidemiology, ecological studies can be a useful method of exclusion of risk and protective factors, but their results must be definitely further investigated in individual-based studies. The multiple sclerosis (MS) prevalence in a small highrisk area gives a special opportunity to apply this intermediate step.

Methods: The MS prevalence 2002 in 16 municipalities of the high-risk county of Värmland, as reported by Callander (2006), were correlated with a number of variables, partly from an earlier period reflecting the childhood of MS patients, and partly from the era of prevalence. Univariate rank correlation analyses and multivariate factor analysis were applied.

Results: The MS prevalence 2002 was correlated $(\mathrm{p}<0.05)$ with 'low living density 1960' (rusticity), 'swine rearing 1968', and 'timber stock (by surface area) 2001'. In factor analysis, two factors were allowed. Only the first factor (eigenvalue 3.236; explaining $53.9 \%$ of total variance) was loaded by the MS prevalence $(\mathrm{p}<0.01)$ and, in addition, by 'rusticity 1960' $(\mathrm{p}<0.05)$, 'swine rearing 1968' ( $\mathrm{p}<0.01)$, and 'timber stock 2001' ( $\mathrm{p}<$ 0.002 ). The second factor (eigenvalue 0.585 ; explaining $9.8 \%$ of total variance) was loaded by 'surface area 1960' (inverse) ( $\mathrm{p}<$ $0.002)$, 'sheep rearing 1977 ' ( $\mathrm{p}<0.002)$, and also by 'swine rearing 1968', but was unrelated to MS (not significant).

Conclusions: The variables loading in factor analysis with the MS prevalence support earlier hypotheses on a role of dietary, pork-related factors and (coniferous) wood in the aetiology of MS, and deserve further investigation in case-control and cohort studies.

\section{P8/41 \\ Gender Ratio by Year of Birth in the National Swedish MS Register}

\author{
Boström I. ${ }^{1}$, Landtblom A.-M. ${ }^{1}$, Stawiarz L. ${ }^{2}$ \\ ${ }^{1}$ University of Linköping, Linköping, Sweden, ${ }^{2}$ Karolinska \\ University, Stockholm, Sweden
}

Background: Research groups from Canada have recently reported a female to male ratio of MS patients continuously increasing during the last 80 years. Also in South East Wales it have been reported an increasing gender ratio.

Aim: To analyse the gender ratio in Swedish MS patients by year-of-birth since 1931.

Methods: Data of 8508 patients with MS i.e. clinical definite MS, born between 1931 and 1980, were extracted from the Swedish MS Register (of total $\mathrm{N}=9392$ registered cases). 
Results: Gender ratio by year-of-birth was analysed. The female to male ratio with a mean value of 2.60 was generally stable throughout time. The highest ratio 3.11 was seen among patients born during the period of 1931-35. A peak of 2.97 during $1956-60$ and a smaller peak of 2.77 at $1976-80$ could be seen. There was an increase in incidence in both sexes up to and including 1956-60, albeit the curve for men was a little different.

Conclusion: The graph illustrating the female to male ratio in Sweden did not correspond to the gender ratio data in Canada and in South East Wales. (The female to male ratio in South East Wales based on year of diagnostic). We believe that the increase of incidence may be due to diagnostic procedures. The peak of female to male ratio in MS patients born 1976-80 in the Swedish data could be explained by an earlier onset of MS in women.

\section{P8/42 \\ Validity and Reliability of Persian Version of MSQoL-54}

Ghaem H. ${ }^{1}$, Borhani Haghighi A. ${ }^{2}$

${ }^{1}$ Shiraz University of Medical Sciences, Shiraz, Iran, Islamic Republic of Iran, ${ }^{2}$ Nemazi Hospital, Shiraz University of Medical Sciences, Neurology Department, Shiraz, Iran, Islamic Republic of Iran

Objective: To translate and to test the reliability and validity of 54-item multiple sclerosis quality of life questionnaire ( MSQoL-54) in Iranian MS patients.

Methods: Using a standard ' forward-backward' translation, cognitive debriefing and cultural adaptation procedure, the English version of the MSQoL-54 was translated to Persian ( the Iranian official language). The subjects were Multiple Sclerosis patients referred to Motaharri clinic, Shiraz, South of Iran. The reliability and internal consistency of the questionnaire were assessed by Cronbach's alpha coefficient and Spearman's correlation, respectively. Validity was assessed using convergent validity and factor analysis.

Results: 141 MS patients (female: 106(75.2\%), male:35 $(24.8 \%)$, with a mean \pm SD age of $32.2 \pm 9.8$ years enrolled in the study. Reliability analysis showed satisfactory result (Cronbach's $\alpha=0.962$ ). There were no significant difference between each item and the mean physical and mental scores of MSQoL-54 by sex, marital status, and education. The scaling success rates were $100 \%$ for convergent validity of each scale. Factor analysis was performed to determine that the Persian version is a two-dimensional measure including physical and mental parameters.

Conclusion: The Persian version of the MSQoL-54 questionnaire has a good structural characteristic, is a reliable and valid instrument, and can be used for measuring the effect of MS on the quality of life.

\section{P8/43 \\ Quality of Life in Multiple Sclerosis: The Impact of Disability, Fatigue and Sleep Quality}

Ghaem H. ${ }^{1}$, Borhani Haghighi A. ${ }^{2}$

${ }^{1}$ Shiraz University of Medical Sciences, Shiraz, Iran, Islamic Republic of Iran, ${ }^{2}$ Nemazi Hospital, Shiraz University of Medical Sciences, Neurology Department, Shiraz, Iran, Islamic Republic of Iran

Objectives: The aim of this study was to measure the quality of life in MS patients and the impact of disability, fatigue and sleep quality.

Methods: Data on health - related quality of life (MS QoL54), fatigue severity scale, sleep quality (PSQI) were obtained in 141 MS patients. Epidemiology data concerning MS type, MS functional system score, EDSS, ... of patients were also provided by a qualified neurologist. Pearson $\alpha$ coefficient, Mann-Whitney $\mathrm{U}$ test, Kruskal-Wallis and linear regression were used to analyze the data.

Results: the mean \pm SD age of 141 MS patients was $32.6 \pm 9.6$ year. Among them, 35(24.8\%) were male, and the others were female. $82(58.1 \%)$ of the patients had EDSS score $\leq 2,36(25.5 \%)$ between $2.5-4.5$ and $23(16.3 \%) \geq 5$. PSQI scores showed that $2(1.4 \%)$ of the patients had good sleep, $16(11.3 \%)$ of them moderate sleep and $123(87.2 \%)$ of them had poor sleep .There was a significant high positive correlation between the quality of mental and physical health composite scores $(r=0.791, \mathrm{P}<0.001)$. Linear regression analysis showed that PSQI score, EDSS, and fatigue score were predictors in the model between the quality of physical score and covariates $(p<0.000)$. Linear regression model showed that fatigue score and PSQI were predictors in the model between the quality of mental score and covariates $(\mathrm{p}<0.000)$.

Discussion: MS Patients had poor and moderate quality of mental and physical health. We suggest that these patients require the attention of health care professionals to observe those who may need further psychological support.

P8/44

Anxiety and Depression in Multiple Sclerosis. A Comparative Population-Based Study in Nord-Trondelag County, Norway

Dahl O.-P. ${ }^{1}$, Stordal E. ${ }^{2}$, Lydersen S. ${ }^{3}$, Midgard R. ${ }^{4}$

${ }^{1}$ Sykehuset Namsos, Neurology, Namsos, Norway, ${ }^{2}$ Sykehuset Namsos, Psychiatry, Namsos, Norway, ${ }^{3}$ The Norwegian University of Science and Technology, Cancer Research and Molecular Medisin, Tronheim, Norway, ${ }^{4}$ Molde Hospital, Neurology, Molde, Norway

Background: Anxiety and depression are widely distributed symptoms among MS patients and within the general population.

Objective: We assessed the prevalence of anxiety and depression in the MS population in Nord-Trøndelag County, Norway in comparison with the general county population. 
Methods: We applied the Hospital Anxiety and Depression Scale questionnaire. A cut off of $\geq 8$ in the questionnaire was used to define significant symptoms of anxiety and depression. Fatigue was measured by Krupp's Fatigue Severity Scale, with a mean cut off $>4$.

Results: The questionnaire was completed by 172 MS-patients and 56000 controls. Among women, $29.7 \%$ of the MS-patients reported anxiety, while $17.4 \%$ of the control population reported these symptoms $(p<0.001)$. For men, the prevalence of anxiety was $31.1 \%$ versus $12.1 \%(\mathrm{p}=0.002)$. Depression was reported by $26.2 \%$ of the men with MS compared to $10.8 \%$ of the controls $(\mathrm{p}<0.001)$. The corresponding figures for women were 25.2 $\%$ versus $10.4 \%(p<0.001)$. Anxiety and depression were not correlated with duration of disease or disability measured by the Expanded Disability Status Scale. Among women, fatigue was associated with anxiety $(\mathrm{p}=<0.010)$ and depression $(\mathrm{p}=0.007)$. No such association was found among men.

Conclusion: Anxiety and depression occur more frequent among MS-patients than in the general population. Fatigue was associated with these neuropsychiatric manifestations only in women.

\section{P8/45 \\ Physical Activity of Patients with Multiple Sclerosis in British Columbia, Canada}

Strehlau V., Tremlett H., Rieckmann P.

University of British Columbia, Neurology, Vancouver, Canada

Background: Exercise has been associated with enhanced brain plasticity. Optimization of exercise in MS requires knowledge of baseline capabilities, of which little is known. We aimed to investigate the association between levels of physical activity and employment status in British Columbia (BC), Canada.

Methods: A self-administered International Physical Activity Questionnaire (IPAQ) was mailed to MS patients visiting a BC MS clinic between 2006 and 2008. Activity levels and metabolic equivalent (MET)-minutes per week were calculated following the IPAQ protocol for patients able to walk unaided (EDSS $<6.0$ ). Employment status was defined as 'employed' (full/part-time) or 'unemployed related to MS'; other causes of unemployment were not considered in the analysis. Statistical analysis was conducted using SPSS 16.0. Ethical approval was gained.

Results: Of the 2482 MS patients mailed a questionnaire; $1126(45.4 \%)$ responded. Valid responders $(n=1116)$ were demographically similar to non-responders. In those able to walk unaided $(\mathrm{n}=785), 41.1 \%(\mathrm{n}=323)$ were employed, and $35.8 \%$ $(\mathrm{n}=282)$ were unemployed due to MS. The overall median MET$\mathrm{min} /$ week was higher for employed versus unemployed patients $(\mathrm{p}<0.001)$. This was primarily driven by commute/transportation $(p=0.043)$ and work-related $(p<0.001)$ activities; no differences were observed for household $(\mathrm{p}=0.133)$ or leisure activities $(\mathrm{p}=0.194)$. Gender, age, disability levels or disease duration could not explain these differences.

Conclusion: Physical activity was lower in patients with MS-associated unemployed compared to employed patients. This was unrelated to leisure or household activities. Consideration might be needed to help patients compensate for reduced work and commute-related physical activity associated with loss of employment.

\section{P8/46 \\ Non-Inferiority of Azathioprine Versus Interferon Beta in Relapsing-Remitting Multiple Sclerosis: The Protocol of a Multicenter, Randomized, Single-Blind Independent Italian Trial}

\begin{abstract}
Massacesi L. ${ }^{1}$, Solari A. ${ }^{2}$, Filippini G. ${ }^{2}$, Benedetti M.D. ${ }^{3}$, La Mantia L. ${ }^{4}$, Milanese C. ${ }^{4}$, M.A.I.N. TRIAL GROUP

${ }^{1}$ II Neurology Division, Department of Neurosciences, University of Florence, Florence, Italy, ${ }^{2}$ Unit of Neuroepidemiology, Foundation IRCCS Neurological Institute C. Besta, Milan, Italy, ${ }^{3}$ Section of Clinical Neurology, Department of Neurological and Vision Sciences, Verona, Italy, ${ }^{4} \mathrm{MS}$ Centre, Foundation IRCCS Neurological Institute C. Besta, Milan, Italy
\end{abstract}

Background: Several studies suggested that azathioprine efficacy in reducing both exacerbations frequency and new brain lesions in Relapsing-Remitting Multiple Sclerosis (RRMS) is comparable to that of Interferon beta (IFNB).

Methods: This is a phase III, multicenter, randomised, single-masked, actively controlled, non-inferiority trial directly comparing azathioprine vs IFNB in terms of disease activity, safety profile and impact on quality of life (QOL). RRMS outpatients enrolled from 30 italian MS centres, aged 18-55 years, with at least two clinical relapses in the preceding 2 years and Expanded Disability Status Scale (EDSS) of 1.0-5.5 are randomly assigned to two arms of treatment: azathioprine ( $3 \mathrm{mg} / \mathrm{kg} /$ day) or IFNB (any approved formulation). The primary end point is the number of relapses per patients over the study period. The secondary end-points are MRI changes, clinical details on relapses, EDSS changes, treatment failures, frequency and severity of adverse events, QOL measures.

Results: Clinical evaluations are performed every 3 months, while brain MRI is performed yearly, similarly to usual clinical practice. The study started on February 2007 and will last 3 years. We chose a non-inferiority design, which is highly efficient in improving statistical power by analyzing one side of the sample distribution, requiring a smaller sample size than the 'equivalence' design.

Conclusions: This is an ongoing trial supported by the Italian Drug Agency, which provides grants fo independent research. Should the hypothesized non-inferiority of azathioprine be confirmed, an additional therapeutic option with convenient route of administration and low cost will be available to patients with MS. 
P8/47

\section{The Association of the Multiple Sclerosis Mortality 1965-1994 in Canada with Food Retail Trade and Food-Related Occupations: an Ecological Study}

Lauer K.

Epidemiologist, Griesheim, Germany

Background: Dietary factors were repeatedly reported to play a role in the aetiology of multiple sclerosis (MS), but definite proof is still lacking due to small sample sizes and other methodological impediments. Ecological studies are an important screening method for variables that should later be included into large case-control and cohort studies.

Methods: The mean annual age-adjusted MS mortality 19651994 in 10 provinces of Canada, as reported by Warren et al.2003, were correlated with data on food retail trade and food-related occupations from the census of 1951. In addition, factor analysis was used to reduce the number of correlated variables to independent 'factors', and to disentangle these variables with respect to their true relation to the MS mortality rate.

Results: Altogether 10 out of 23 variables from food retail trade and food-related occupations were significantly correlated $(\mathrm{p}<0.05)$ with the MS mortality. Annual income, urbanity and an 'industry score' were additionally associated. In factor analysis, the age-adjusted MS mortality loaded significantly on one factor (eigenvalue $0.887 ; 7.4 \%$ of total variance) together with the number of 'fishermen' (inverse) and 'fish canners' (inverse), sale in 'meat stores', 'eating places', 'delicatessen stores', 'egg and poultry stores', and 'income' (all by population 1951). 'Bakeries', 'tobacco shops', 'urbanity' and 'industry score' loaded on another factor (eigenvalue $8.638 ; 72.0 \%$ of total variance), that was, however, unrelated to the MS mortality.

Conclusions: The MS-associated food variables are partly in line with prior hypotheses, and should be tested in forthcoming large case-control and cohort studies.

\section{P8/48}

\section{Acute Cardiovascular Dysfunction in Multiple Sclerosis: Report of Two Cases and Literature Review}

Vedovello M. ${ }^{1}$, Invernizzi P. ${ }^{2}$, Rossi $F .1$, Turatti M. ${ }^{1}$ '
Gajofatto A. ${ }^{1}$, Toniolo M. ${ }^{3}$, Vassanelli $C .{ }^{3}$, Morando ${ }^{3}{ }^{,}$
Benedetti M.D. ${ }^{1}$
${ }^{1}$ University of Verona, Neurology, Verona, Italy, ${ }^{2}$ Istituto
Ospedaliero Fondazione Poliambulanza, Neurology,
Brescia, Italy, ${ }^{3}$ University of Verona, Cardiology, Verona,
Italy

Background: Cardio-pulmonary dysfunction is an unusual event in multiple sclerosis (MS). The most common pathogenetic mechanism is mitoxantrone toxicity, but a few cases are reported in which cardiac involvement was associated to brainstem lesions.
Methods: We describe two MS cases with acute cardiac dysfunction not related to mitoxantrone toxicity. A young woman followed for relapsing-remitting (RR) MS was admitted to the Emergency Department for shock state, dyspnea, hypothermia, severe hypotension and tachycardia. Transthoracic echocardiography revealed left ventricular ejection fraction reduced to $10 \%$. A 35-year old woman with RR-MS developed right limbs sensory symptoms and right oculomotor nerve palsy. She started intravenous methylprednisolone, but on day 4th she developed dyspnea and sinus bradycardia (HR 38/min), lasting two days.

Results: In our first case, laboratory and instrumental signs of myocarditis were investigated, but serology for infections, rheumatologic markers, cardiac MRI and coronary angiography were normal. After inotropic therapy and intravenous immunoglobulin she fully recovered. Magnetic Resonance Imaging (MRI) two months later revelead a new lesion in posterior medulla. In the second case blood tests were normal. Two weeks later brain MRI showed a new enhancing lesion in the pons-midbrain junction.

Conclusions: In the first case the new brainstem MRI lesion probably involved the dorsal vagus nucleus and determined a cardiogenic shock. Although in our second case methylprednisolone cardiac effect cannot be excluded, acute demyelination in the brainstem might have interfered with pons and midbrain regulatory nuclei, resulting in bradycardia.

\section{P8/49 \\ Incidence of Pediatric Multiple Sclerosis (MS) in Sardinia, Insular Italy (1968-2007) \\ Pugliatti M. ${ }^{1}$, Cossu P. ${ }^{1}$, Leoni S. ${ }^{1}$, Sotgiu S. ${ }^{1}$, Manca S. ${ }^{2}$, Rosati . $^{1}$ \\ ${ }^{1}$ Department of Neurological Sciences, University of Sassari, Sassari, Italy, ${ }^{2}$ Department of Child \\ Neuropsychiatry, Sassari University Hospital, Sassari, Italy}

Sardinia is among the regions at highest risk for MS worldwide. Early childhood can be the age of susceptibility to MS in Sardinians. The incidence of MS in the Sardinian population aged $<18$ years was assessed by revising clinical and demographic multiple sources (Sassari province, 450,000 pop). In 1968-2007, 94 individuals (72 females, 22 males) had clinical onset of MS in the study area. The mean annual incidence rate was $0.54(95 \% \mathrm{CI}$ : $0.51-1.05)$ per $100,000,0.03(0.02-0.04)$ in males and $0.82(0.74-$ $1.56)$ in females. Of these, $98 \%$ had onset in post-pubertal age $(>9$ years); only two males had onset during pre-puberty. The highest rate was observed in individuals aged 15 years and older ( 7.6 per 100,$000 ; 3.5$ in males and 11.9 in females). Incidence fluctuated over study period with a peak of 1.2 in $1978-87$ due to increased number of only female cases. The distribution by gender changed significantly over time, with a gradual drop of the F:M ratio to 0.8 in 1998-2007 (overall mean ratio: 3.3). Age of onset did not change over time. Children presented with sensory (36\%), visual $(33 \%)$, motor (14\%) and other (18\%) symptoms. MS had relapsing-remitting initial onset in $97 \%$ of cases. Incidence of pediatric MS in Sardinians appears to be higher than reported elsewhere.

Acknowledgements: for financial support: Regione Autonoma Sardegna (Assessorato Igiene, Sanità e Assistenza 
Sociale, 2005); FISM (grants 69/R/97, 14/R/2007, 19/R/2008); Dr. PG Annicchiarico and coll. (SSI ASL 1 Sassari) for assistance in registry-based data collection.

\section{P8/50 \\ A Case of Marburg's Variant Multiple Sclerosis with Long Survival}

Turatti M., Rossi F., Gajofatto A., Vedovello M., Benedetti M.D.

University of Verona, Verona, Italy

Background: Marburg's disease is defined as an atypical form of multiple sclerosis, first described by Marburg in 1906, characterized by rapid and aggressive clinical course and large demyelinating lesions in the central nervous system, usually leading to death in short time.

Methods: We describe a case presenting with motor and sensory symptoms suggestive of acute myelopathy, rapidly followed by cerebellar dysfunction and consciousness impairment. Clinical, laboratory and radiological evaluations suggested a central nervous system demyelinating disease.

Results: The patient was diagnosed with Marburg's variant multiple sclerosis. The patient underwent different treatments, including steroids, cyclophosphamide, plasma-exchange and lastly interferon beta. He reached a clinical stability which persists at 3 years from onset.

Conclusions: Marburg's type multiple sclerosis has been typically considered a fatal illness within few weeks, but our case, in addition to the only other published case with a favourable outcome, suggests that patients with this condition might reach a long clinical stability, even though residual neurological disability is severe.

\section{P8/51}

\section{Factors Predicting Multiple Sclerosis Relapse} Location: A Prospective Study

Naldi P. ${ }^{1}$, Collimedaglia L. ${ }^{2}$, Bonissoni S. ${ }^{3}$, Ruggerone S. ${ }^{1}$, Vecchio D. ${ }^{2}$, Rosso M.G. ${ }^{4}$, Monaco F. ${ }^{2}$, Perla F.

Leone M.A. ${ }^{1}$

${ }^{1}$ Clinica Neurologica, Ospedale Maggiore della Carità,

Novara, Italy, ${ }^{2}$ Clinica Neurologica, Università del Piemonte Orientale, Novara, Italy, ${ }^{3}$ Ospedale S. Corona, Pietra

Ligure, Italy, ${ }^{4}$ Ospedale SS Croce e Carle, Cuneo, Italy

Background: Location and severity of relapses in multiple sclerosis (MS) vary from patient to patient. We aimed to prospectively evaluate predictors of relapse location and severity of the second attack in a cohort of MS patients.

Methods: Ninety-five consecutive patients with a first demyelinating event were recruited in two first-referral Hospitals from January 2001 to December 2003 and followed-up to July 31,
2005. Severity of the relapse was graded according to the difference between the scores at the day of maximum worsening and before the onset of the relapse, as: very mild-VM (0-1 point in one FS only), mild-M (2 points in one FS or 1 point in two FSs), moderate-MD ( 3 points in one FS or 2 in one FS and 1 in another, or 1 point in three FSs), severe-S (any other combination exceeding moderate).

Results: Sixty-four patients had a second demyelinating event. Patients with a S, MD, M first attack had a higher risk to have a $\mathrm{S}, \mathrm{MD}, \mathrm{M}$ second relapse compared to $\mathrm{VM}$ (Odds Ratio $=6.5,95 \%$ Confidence Limits $=2.2-18.3$ ). Patients with bior poly-symptomatic first attack had a higher risk of a bi- or polysymptomatic second attack (3.1, 1.1-9.1). Second attacks had a higher risk to localize in the same FS as the first attacks: motor (3.1, 1.1-8.9), sensitive (3.9, 1.4-11.3), visual (5.8, 1.6-21.4), cerebellar (7.8, 1.4-45.2), brainstem (3.4, 0.9-13.2).

Conclusions: the second attack shows a tendency to repeat localization and severity of the first attack in MS patients.

\section{$\mathrm{P} 8 / 52$ \\ Desmopressin-Associated Water Intoxication in a Patient with Multiple Sclerosis}

\author{
Giannikopoulos G., Stamoulis I., Karamouzos E., \\ Ververaki S., Tsouni P., Ydreos I., Tripodaki E., Giannoulos I., \\ Zorzou M.-P. \\ General Hospital of Chios, Chios, Greece
}

Background: To present a case of hyponatremia in a patient with multiple sclerosis.

Methods: A 44-year old patient from Norway was admitted to the hospital complaining of fatigue, dizziness and headache of recent onset. She had an 8-year history of multiple sclerosis. She reported receiving no medication. Her physical examination was unremarkable. The laboratory workup revealed only severe hyponaetremia $\left(\mathrm{Na}^{+} 111 \mathrm{mmol} / \mathrm{L}\right)$. After repetitive questions concerning medications the patient recalled receiving nasal desmopressin for the control of nocturia. Hyponatremia was due to increasing water intake in order to avoid dehydration throughout her summer holidays. The diagnosis of desmopressin-induced water intoxication was presumed. She received 1lt of normal saline iv daily, furosemide iv $20 \mathrm{mg}$ bid and fluid restriction. Her clinical picture improved soon after sodium increase.

Results: Desmopressin is an analogue of antidiuretic hormone indicated for the treatment of central diabetes insipidus, primary nocturnal enuresis and in bleeding disorders. Hyponatremia is a rare adverse event ( 1 every 10.000 patients). Two distinct mechanisms have been proposed: the first refers to otherwise stable patients, with an extrinsic factor (most likely increased water intake) interfering with desmopressin and resulting in water intoxication. The second mechanism is probably attributed to idiosyncratic factors resulting in severe hyponatremia during the first 3 weeks of desmopressin initiation.

Conclusions: Patients receiving desmopressin, should be cautious to the possible appearance of hyponatremia. According to the FDA safety labeling revision, desmopressin therapy should be interrupted during acute illnesses that interfere with fluid bal- 
ance and other conditions that increase water consumption (e.g. hot weather).

\section{LB-P8/92 \\ Increasing Frequency of Multiple Sclerosis in Catania, Sicily: A $\mathbf{3 0}$ Years Survey}

Nicoletti A. ${ }^{1}$, Patti F. 1, Lo Fermo S. ${ }^{1}$, Messina S. ${ }^{1}$, Bruno E., Quattrocchi G. ${ }^{1}$, Laisa P. ${ }^{1}$, Cilia S. ${ }^{1}$, Roberto M. ${ }^{2}$,

Maimone D. ${ }^{3}$, Zappia M. $^{1}$

${ }^{1}$ Department of Neurosciences, University of Catania, Catania, Italy, ${ }^{2}$ Cannizzaro Hospital, Neurology Division, Catania, Italy, ${ }^{3}$ Garibaldi Hospital, Neurology Division,

Catania, Italy

Objective: To determine the incidence of multiple sclerosis (MS) and its temporal profiles from 1975 to 2005 in the city of Catania.

Methods: Incidence of MS from 1975 to 31 December 1999 was previously investigated by our group. We studied the frequency of MS in the community of Catania from 1 January 2000 to 31 December 2004 in a population of 313,110 inhabitants (2001 census).

The primary sources for the case ascertainment were the neurological and motor-rehabilitation departments, the MS Centers, the Italian MS Association, private neurologists and family doctors. We considered as incident cases all patients who had experienced the onset of MS during the study period and fulfilled the Poser's criteria.

Results: From 2000 to 2004, 108 patients (with MS had the clinical onset of the disease;

The mean annual incidence was $7.0 / 100,000$ (C.I. 95\%5.713.7) and was higher in women

$(8.4 / 100,000 ; 95 \%$ C.I $6.4-10.5)$ than in men $(5.3 / 100,000$; CI $95 \% 3.7-7.2$ ) with a sex ratio (female/male) of 1.7 . The mean Lag-time was $1.4 \pm 1.7$.

During the last 30 years the incidence of MS in our population increased from 1.8/100,000 during the first quinuennium 19741979 to $7.0 / 100,000$ during 2000-2004. Incidence risk recorded in this last quinquennium is still increased from the risk recorded during 1995-1999 (5.5/100,000; 95\%CI 4.4-6.7).

Conclusions: Incidence rates have further increased in our population suggesting a possible increasing risk of MS our population.

\section{LB-P8/98 \\ The Course of Primary Progressive Multiple Sclerosis is Worse in Brazilians of African Descent}

\author{
Vasconcelos C.C.F.', Thuler L.C.S. ${ }^{1}$, Santos G.A.C. ${ }^{1}$, \\ Papais Alvarenga M. ${ }^{1}$, Alvarenga M.P. ${ }^{1}$, \\ Gomes Camargo S.M.G. ${ }^{2}$, Alvarenga R.M.P. ${ }^{1}$ \\ ${ }^{1}$ Universidade Federal do Estado do Rio de Janeiro, \\ Neurologia, Rio de Janeiro, Brazil, ${ }^{2}$ Hospital da Lagoa, \\ Neurologia, Rio de Janeiro, Brazil
}

Studies published suggest that the clinical evolution of the multiple sclerosis is worse in individuals of African descent ${ }^{1-3}$. Possible differences in the progression of disability between Brazilians of African descent and whites with primary progressive MS (PPMS) have yet to be evaluated.

Objective: to assess differences in the progression of the disease according to ethnic group.

Material and Methods: Sixty PPMS patients with at least five years of the disease were analyzed. According to existence of black individuals in the family up to the third generation, patients were classified as being of African descent. Progression was measured as the time in years to reach scores 3, 6 and 8 of Kurtzke's Scale (EDSS).

Results: $56 \%$ women, $61.6 \%$ whites (37/60) and $38.3 \%$ Brazilians of African descent (23/60); 91\% of African descent reached EDSS 3 after a mean of 2 years of the disease and $94.5 \%$ of the whites aftern a mean of 3 years $(p=0.04) ; 78 \%$ patients of African descent reached EDSS 6 after a mean of 3.8 years and $75.6 \%$ of white patients after a mean of 5.7 years $(\mathrm{p}=0.02)$; EDSS 8 was reached by $17 \%$ of Brazilians of African descent after a mean of 5 years, and $43 \%$ of whites after a mean of 9.8 years $(\mathrm{p}=0.08)$.

Conclusions: The poorer and more rapid evolution seen in these Brazilian patients of African descent, as also reported for Americans and Europeans of African descent, suggests a stronger ethnic rather than environmental effect on the progression of multiple sclerosis.

\section{LB-P8/99 \\ Survival Analysis and Intervention Analysis of Retrospectively Observed Multiple Sclerosis Relapse Rate}

\author{
Oikonen M. ${ }^{1}$, Erälinna J.-P. ${ }^{2}$ \\ ${ }^{1}$ University of Turku, Aerobiology Unit, Turun Yliopisto, \\ Finland, ${ }^{2}$ Department of Neurology, Turku University \\ Hospital, Turku, Finland
}

Background: Retrospective Multiple Sclerosis (MS) relapse occurrence data was studied by applying Kaplan-Meier survival analysis and ARIMA (Autoregression Integrated Moving Average) intervention analysis.

Methods: MS exacerbations from January 1995 - March 2001 were observed retrospectively of 309 MS patients with regu- 
lar visits at the Department of Neurology of the Turku University Hospital in Turku, Southwestern Finland. 116 patients had used interferon (21 Avonex, 22 Betaferon and 73 Rebif). Strict criteria were enforced for interferon treatment and neurological examinations were repeated annually. Relapse-free time in days were calculated from interferon beginning and in non-interferon treated patients from diagnosis and compared with survival analysis. The change in monthly relapse rate during the first 12 months of interferon use was compared to the 24 months before starting interferon with an ARIMA $(0,1,1)$ model.

Results: The relapse-free time was longer during interferon treatment compared to non-treated patients. No differences were detected between the interferon groups, but the groups were unequally censored. The total reduction in relapse rate during the first three months of interferon treatment was $-11 \%$ as compared to the time before treatment, and the relapse rate remained at the lower level until the end of the 12-month period.

Conclusions: Irregular relapse intervals can be studied with Kaplan-Meier survival analysis. ARIMA intervention analysis is a flexible tool for measuring treatment effect in autoregressive time-series. Reliable hospital records on MS relapse occurrence can be a cost-effective and ethically sound source of long-term patient data for specific time-series analyses.

\section{P9}

\section{Neurological Aspects of Aging}

\section{$\mathrm{P} 9 / 53$ \\ Cognitive Function and Nutrient Intakes in Community-Dwelling Elderly People of Low Socioeconomic Status}

German L. ${ }^{1}$, Kahana C. ${ }^{2}$, German I. ${ }^{3}$, Shahar D.R. ${ }^{2}$

1 University Ben-Gurion, Epidemiology and Health Services Evaluation, Beer-Sheva, Israel, ${ }^{2}$ The S.Daniel International

Center for Health and Nutrition, Beer-Sheva, Israel,

${ }^{3}$ Soroka Medical Center, Beer-Sheva, Israel

Background: People of low socioeconomic status (LSES) are known to suffer greater nutritional risk and may have higher rates of cognitive impairment as well.

Study objective: To examine the relation between cognition and nutrient intakes of LSES community-dwelling elderly.

Methods: A neighbourhood cluster sampling based on a list of welfare recipients was used to obtain a representative sample; 111 people aged $60+$ were enrolled.

The cognitive status was assessed with the Mini-Mental State Examination (MMSE), dietary pattern was evaluated using by 24-hour dietary recall.

Results: The prevalence of cognitive impairment (MMSE $<$ 24 ) in the studied population was $49.5 \%$. Although higher intakes of vitamin $\mathrm{B}_{1}, \mathrm{~B}_{2}, \mathrm{~B}_{6}$ and $\mathrm{C}$, magnesium and fiber were weakly associated with better cognitive function, significant differences between participants with vs without cognitive impairment were found in vitamin $\mathrm{B}_{6}$ consumption:0.8(0.4) vs1.1 (0.4) and fiber intake:10.5(5.6) vs12.9(5.8) respectively. Compared to elderly with mild cognitive impairment (MMSE 18-23), participants with severe (MMSE 0-17) cognitive impairment reported higher consumption of sodium and lower vitamin $\mathrm{B}_{6}$ intake. In elderly with mild cognitive impairment higher intakes of vitamin E and PUFAs were significantly correlated with better cognition (Spearman's $r$ 0.36 and 0.38 respectively).

Conclusions: LSES elderly people suffer from high rates of cognitive impairment that were associated with nutritional deficiencies. The findings suggest that health care system and social service should pay great attention to

LSES community-dwelling elderly to increase nutrient intakes and reduce the prevalence of nutrient inadequacy. In addition, prospective studies are needed to clarify the temporal relationship between the cognitive and nutritional domains.

\section{P11}

Neuropsychology
P11/54

Neuropsychological Deficits and Functional Abilities Five-Years Post Subarachnoid Haemorrhage

Chahal N.1, Barker-Collo S.1, Feigin V.2

${ }^{1}$ Unversity of Auckland, Department of Psychology and Clinical Research Trials Unit, Auckland, New Zealand,

${ }^{2}$ University of Auckland, Clinical Research Trials Unit, Auckland, New Zealand

Background: While neuropsychological deficits have been the focus of research in subarachnoid haemorrhage (SAH) patients, there is a lack of population based studies on long-term neuropsychological and functional impairment in SAH survivors.

Objectives: To report on and examine the relationship between areas of neuropsychological deficits and functional abilities/quality of life of a population based sample of 5-year survivors of $\mathrm{SAH}$ and to contrast their performance to a group of matched controls.

Method: This was a cross-sectional examination of long-term (5-years) SAH outcomes. Assessment included measures of neuropsychological functioning, neurological impairment, disability, handicap, and health related quality of life domains. Participants include 32 5-year survivors of SAH who were previously enrolled in the Auckland Regional Community Stroke Study (ARCOSS) (2002-2003) and 32 healthy controls matched in terms of age, gender, and ethnicity.

Results: Neuropsychological (e.g., memory, language, visuoperceptual reasoning, processing speed, and executive func- 
tioning) and functional impairments (neurological impairment, disability, handicap, and health related quality of life) in SAH survivors, 5-years post stroke are compared to performance of matched healthy controls. The independent contribution of neuropsychological functioning to predict quality of life over and above that of physical impairment is also examined.

Conclusion: The results of the study will lead to better understanding of the dynamics of long term recovery after SAH, and establish the importance of various neuropsychological factors to overall outcomes; thus help in designing appropriate rehabilitation programs.

\section{P13}

\section{Neuromuscular Disorders}

P13/55

Prevalence of Non-Specific Back Pain in a Rural Area in Northern Tanzania: A Community-Based Door-to-Door Survey

Dent W. ${ }^{1}$, Stelzhammer B. ${ }^{2}$, Kerschbaumsteiner K. ${ }^{2}$, Meindl M. ${ }^{2}$, Kaaya J. ${ }^{3}$, Schmutzhard E. ${ }^{2}$, Winkler A.S. ${ }^{4}$

${ }^{1}$ Department of Neurosurgery, University Hospital of Zürich, Zurich, Switzerland, '2Department of Neurology, Medical University of Innsbruck, Innsbruck, Austria, ${ }^{3}$ Babati District Hospital, Manyara, Tanzania, United Republic of, ${ }^{4}$ Ludwig-Maximilians-University, Interdisciplinary Centre for Palliative Care and Department of Neurology, Munich, Germany

Background: Back pain (BP) is the most prevalent musculoskeletal condition and one of the most common causes of disability in the developed world. Anecdotally, there is a general assumption that BP prevalence in Africa is comparatively lower than in developed countries. The aim of this survey was to assess the prevalence of non-specific overall BP in a rural African population in northern Tanzania.

Methods: From December 2003 until June 2004 a door-todoor study including 1,192 households with 7,412 people was done. After a screening dialogue with a representative family member, a face-to-face interview with the affected subject was performed, using a standardized and validated questionnaire.

Results: Three hundred ninety-four subjects met the case definitions of BP resulting in a crude lifetime prevalence of 5.3\% (95\% CI 4.8-5.9\%; age-adjusted prevalence rate: 9.1\%). Females were affected twice as often $(7.1 \%$, CI $6.3-8.0 \% ; 261 / 3,681)$ than males $(3.6 \% ; 3.0-4.2 \% ; 133 / 3,731$; male to female ratio: $\mathrm{p}<$ $0.001)$. There was a steady increase of BP with age resulting in prevalence rates of $23.1 \%$ in the seventh and $24.7 \%$ in the eighth decade. The peak prevalence rate of $29.6 \%$ was seen in people aged 80 years and older. The overall point prevalence was $3.2 \%$ (95\% CI 2.8-3.7\%; age-adjusted prevalence rate: 5.8\%).
Conclusions: This is the first 'community-based' BP prevalence survey in a rural population of East Africa. The study shows that in our population BP is less common compared to other African countries and by far less common compared to industrialized countries. Potential causes will be discussed.

\section{P13/56 \\ Population-Based Epidemiologic Study of Neurological Diseases in Taiwan Series II: Myasthenia Gravis and Wilson's Disease}

\author{
Lai C.-H. ${ }^{1}$, Tseng H.-F.T. ${ }^{2}$ \\ ${ }^{1}$ Department of Neurology, Changhua Christian Hospital, \\ Changhua, Taiwan, Republic of China, ${ }^{2}$ Department of \\ Research and Evaluation, Southern California Permanente \\ Medical Group, Pasadena, USA
}

Background: The purpose of the study is to investigate the epidemiology of Myasthenia gravis (MG) and Wilson's disease, two diseases in the list of Serious Accidents and Diseases, in Taiwan.

Methods: Cases of MG and Wilson's disease were identified according to corresponding International Classification of Diseases, Ninth Revision, codes from the National Health Insurance Research Database from January 2000 to December 2005. Age and sex specific incidences of these diseases were estimated by dividing the incidence number by population data obtained from the Department of Statistics, Ministry of the Interior.

Results: During the study period, 4,266 cases of MG were identified. The male to female ratio was 0.7 . The average annual incidence was 2.1 per 100,000 . The age specific incidence of MG approximately increased with age from age group of 10-14 to age group of 65-69 for male and 70-74 for female, and then declined. There were 495 cases of Wilson's disease. Male to female ratio was 1.28 . The average annual incidence was 0.27 per 100,000 . The incidence of Wilson's disease peaked at age group of 15-19. The prevalence of both MG and Wilson's disease increased steadily during the study period.

Conclusion: This is the first population-based epidemiologic data of MG and Wilson's disease in Taiwan. Compared with previous epidemiologic studies, the present study shows higher incidence of MG, which may result from better ascertainment. The medical expenses of these two diseases are expected to increase over time due to gradually increased prevalence. 
P13/57

\section{Prevalence of Myopathic Patients in Akita Prefecture in Northern Japan}

Kobayashi M. ${ }^{1}$, Sugawara M. ${ }^{2}$, Abe E. ${ }^{1}$, Obara K.1, Mamiya S. ${ }^{1}$, Ohnishi H. ${ }^{2}$, Toyoshima I. ${ }^{2}$

${ }^{1}$ National Hospital Organization Akita National Hospital, Yurihonjo, Japan, ${ }^{2}$ Akita University School of Medicine,

Akita, Japan

Background: It is preferable that many patients are able to participate in clinical trials of myopathy that are expected to be conducted in the near future. As a first step, survey of the current status of myopathic patients is needed.

Methods: A questionnaire survey of the number of myopathic patients and the grounds for the diagnosis was sent to a total of 134 departments at hospitals listed in the directory of Akita Medical Association.

Results: Responses were received from $70 \%$ of departments. The total number of myopathic patients was 217 . The first was 65 myotonic dystrophy (MD), 42 inflammatory myopathies, 26 Duchenne muscular dystrophy (DMD), 13 limb-girdle muscular dystrophy, 12 mitochondrial myopathies, 10 congenital myopathies, 9 congenital muscular dystrophy, 8 distal myopathy with rimmed vacuole, 7 distal muscular dystrophy (Miyoshi), 5 facioscapulohumeral muscular dystrophy, and 4 Becker type progressive muscular dystrophy. The prevalence of MD was 5.8, and that of DMD was 2.3 (cases per 100,000 people). Deletion/ duplication was only found in $6(23 \%)$ of the DMD patients. The prevalence of DMD (per 100,000 men) was 9.3 in 1-10 years old, 11.1 in 11-20 years old, 16.4 in 21-30 years old, and 11.1 in 31-40 years old.

Conclusions: The prevalence of myopathies in Akita Prefecture was similar to that described in previous reports. But DMD patients appeared to decrease. Accurate definite diagnosis is necessary for their participation in clinical trials.
P13/58

\section{Epidemiology of Hereditary Myotonic Dystrophy in Populations of Some Provinces in Mongolia}

\author{
Baasanjav D., Ya E. \\ Mongolian Medical Research Institute, Neurology, \\ Ulaanbaatar, Mongolia
}

Background: Epidemiological studies of hereditary neurological diseases in Mongolia are being conducted since 1997. This study introduces myotonic dystrophy spread among the population of seven out of 21 provinces in the country.

Method: Diagnosis was established by neurological clinic and hereditary anamnesis using special questionnaire form working out by except group.

Result: The number of patients and family with Myotonic dystrophy and its prevalence for 100.000 population in every and total for all adducing below.

Conclusion: The prevalence of myotonic dystrophy per 100.000 of population varies widely: from 0 (Dornod) to 15,35 (Khuvsgul), a general prevalence among total population studied being 5,76 . The total number of patients was 64 , living in 20 households. This is relatively high rate, despite the uneven distribution among localities. However, more precision diagnosis is required in order to reveal the prevalence that would be comparable to international standards.

Table for P13/58

\begin{tabular}{llccc}
\hline Provinces and their population & Year of investigations & Number of households & Number of patients & Prevalence for 100.000 \\
\hline 1. Khovd pop. 91500 & 2000 & 1 & 3 & 3.28 \\
2. Dornod pop. 68200 & 2002 2004 & - & - & - \\
3. Selenge pop. 92834 & 2002 & 3 & $30(\mathrm{~m}=18 ; \mathrm{f}=12)$ & 7.54 \\
4. Ulaanbaatar- capital pop. 605292 & 1997 2000 & 11 & $19(\mathrm{~m}=7 ; \mathrm{f}=12)$ & $15.35(\mathrm{~m}=11.10 ; \mathrm{f}=19.77)$ \\
5. Khuvsgul pop.123740 & 2005 & 2 & $2(\mathrm{~m}=2 ; \mathrm{f}=0)$ & 2.45 \\
6. Uvs pop. 81609 & 2005 & 2 & $3(\mathrm{~m}=1 ; \mathrm{f}=2)$ & $6.27(\mathrm{~m}=4.26 ; \mathrm{f}=8.22)$ \\
7. South Gobi pop. 47821 & 2005 & 1 & 64 & 5.76 \\
Total pop. 1.110996 & & 20 & & \\
\hline
\end{tabular}




\section{P14}

Parkinson's Disease and Other Movement Disorders

P14/59

\section{Adherence to the Antiparkinsonian Drug Treatment: The CUMPLIPARK Study}

Martinez-Martin P.1, Balaguer-Martinez E. ${ }^{2}$, Campos Arillo V. ${ }^{3}$, Grandas-Perez F. , The CUMPLIPARK Study Group

${ }^{1}$ National Center for Epidemiology and CIBERNED, Carlos III Institute of Health, Area of Applied Epidemiology, Madrid, Spain, ${ }^{2}$ Department of Neurology, Hospital General de Catalunya, Barcelona, Spain, ${ }^{3}$ Department of Neurology, Xanit International Hospital, Benalmadena, Spain, ${ }^{4}$ Department of Neurology, Hospital Gregorio Marañón, Madrid, Spain

Background: Some studies have demonstrated frequent problems of treatment adherence in patients with PD. Present study was aimed at determining the treatment adherence in PD patients assisted in Departments of Neurology across Spain.

Methods: Each participating neurologist included five consecutive patients who were receiving stable treatment for PD. Pill counts, Morisky questionnaire, and electronic drug monitoring bottles were used for controlling medication adherence during a 3-month follow-up period. Demographic data were recorded. A survey related to experience in clinical practice and estimations of treatment compliance was completed by the participant neurologists.

Results: 108 neurologists included 549 PD patients (531, $96.7 \%$ fully computable) in the study. Mean $( \pm \mathrm{SD})$ age was $68.9 \pm 10.1$ years; $57.3 \%$, males; $10.9 \%$ illiterates; $75.3 \%$ in Hoehn and Yahr stage II or III. As per the SPMSQ, patients with cognitive impairment were not included. At 3 months follow-up, $71.3 \%, 43.2 \%$, and $95.0 \%$ of patients were not adherent as per the aforementioned monitoring procedures. There were not significant differences in adherence level by gender, age, or number of anti-parkinsonian drug treatments. Neurologists $(64.5 \%$ with $\geq 10$ years as practitioners; $64.1 \%$ attending regularly $\geq 100$ PD patients) estimated that less than $30 \%$ of patients would be moderately or importantly non-adherent (compliance $\leq 20 \%$ of the doses). Regular planned visits (98.1\%) and one-daily dose drugs were considered by doctors the most efficacious way to assure treatment adherence.

\section{Conclusion:}

1) Adherence to drug treatment is low in PD patients;

2) Neurologists opinion about therapeutic compliance by patients is not realistic;

3) Adherence should be closely monitored.

\section{P14/60 \\ Impaired Olfaction and Colour Discrimination in Genetic and Non-Genetic Parkinson Disease}

Kertelge L. ', Brüggemann N. ${ }^{1}$, Schmidt A. ', Tadic V. ${ }^{1}$, Wisse C. ${ }^{1}$, Drude L. ${ }^{1}$, van der Vegt J. ${ }^{2}$, Schneider S.A. ${ }^{1}$, Siebner H. ${ }^{2}$, Lohmann K. ${ }^{1}$, Buhmann C. ${ }^{3}$, Hagenah J. ${ }^{1}$, Klein C. ${ }^{1}$, Kasten M. ${ }^{4}$

${ }^{1}$ University of Luebeck, Neurology, Luebeck, Germany, ${ }^{2}$ University of Kiel, Neurology, Kiel, Germany, ${ }^{3}$ University of Hamburg, Neurology, Hamburg, Germany, ${ }^{4}$ University of Luebeck, Psychiatry and Psychotherapy, Luebeck, Germany

Background: Impaired olfaction is typical of idiopathic Parkinson disease (IPD), but its potential role is uncertain in genetic (GPD) and atypical PD (APD). Diminished colour discriminiation has been suggested as another early sign of a dopaminergic deficit but has not been systematically studied to date. Furthermore, it is unknown whether both are linked.

Methods: We examined 88 IPD, 43 GPD (23 affected, 18 unaffected mutation carriers), 8 APD, recruited from consecutive outpatients and family studies, and 96 unrelated controls. All participants underwent a standardised neurological examination, the University of Pennsylvania Smell Identification Test (UPSIT) and the Farnsworth-Munsell Hue (FM) colour discrimination test. Participants were tested for mutations in the Parkin and PINK1 and for known mutations in the SNCA and LRRK2 genes.

Results: See table below.

High UPSIT percentiles (age- and gender-adjusted) $=$ good olfaction, high FM error score = poor colour discrimination.

Using unadjusted UPSIT and FM scores, both were correlated $(\mathrm{r}=-0.205, \mathrm{p}=0.003$, Spearman-Rho test) in the entire and the IPD group $(\mathrm{r}=-0.308, \mathrm{p}=0.01)$.

Conclusions: First, we confirmed olfaction and colour discrimination to be impaired in IPD. Second, in APD, olfaction was better than in IPD but colour discrimination was severely impaired. Third, olfaction appears to be independent of clinical status of mutation carriers, suggesting it to be a premotor sign of PD. Colour discrimination varied widely obscuring group differences.

Table (for P14/60) UPSIT percentiles and FM error score

\begin{tabular}{|c|c|c|c|c|c|c|}
\hline & IPD & GPD, affected & GPD, unaffected & APD & Controls & p-value* \\
\hline
\end{tabular}

Mean \pm standard deviation, *two-way ANOVA 


\section{P14/61}

\section{Parkinson Psychosis Rating Scale: Cross- Cultural Evaluation and Content Validity}

Virues-Ortega J. ${ }^{1}$, Rodriguez-Blazquez C. ${ }^{2}$, Michelli F.3, Carod-Artal F.-J. ${ }^{4}$, Serrano-Dueñas M. ${ }^{5}$, Meza-Rojas G. ${ }^{6}$, Ruiz-Galeano G. ${ }^{6}$, Velazquez C. ${ }^{6}$, Martinez-Martin P.7

${ }^{1}$ Consortium for Biomedical Research in Neurodegenerative Diseases (Centro de Investigación Biomédica en Red sobre Enfermedades

Neurodegenerativas - CIBERNED), Madrid, Spain, ${ }^{2}$ CIBERNED, Madrid, Spain, 3Jose de San Martin Clinical Hospital, Department of Neurology, Buenos Aires, Argentina, ${ }^{2}$ Sarah-Brasilia Hospital, Department of Neurology, Brasilia, Brazil, ${ }^{5}$ Carlos Andrade Marín Hospital and Medicine Faculty of Pontifical Catholic University of Ecuador, Movement Disorders Unit, Neurological Service, Quito, Ecuador, ${ }^{6}$ Neurocenter, Central Social Security Hospital, Asunción, Paraguay, ${ }^{7}$ Instituto de Salud Carlos III (National Center of Epidemiology) \& CIBERNED, Madrid, Spain

Background: This study assessed the psychometric attributes of the Parkinson Psychosis Rating Scale (PPRS) in four Latin American countries (Argentina, Brazil, Ecuador, and Paraguay).

Methods: Consecutive PD patients with stable caregiver. In addition to demographic and historical data, Hoehn and Yahr staging, SCOPA-Motor, MiniMental State Examination (MMSE), Hospital Anxiety and Depression Scale (HADS), and Clinical Impression of Severity Index-PD (CISI-PD) were applied.

Acceptability, internal consistency, factor structure, construct validity, and precision were explored. Content validity was determined through the Lynn's method, including 6 experts and the following criteria: (a) proportion of items relevant and concise (criterion $\geq 75 \%$ ), and (b) proportion of experts rating items as relevant and concise (criterion $\geq 85 \%$ )

Results: 388 PD patients were included (mean age, $64.5 \pm 10.7$ years; $59.8 \%$ males; PD duration, $8.2 \pm 4.9$ years; HY, 1 to 5 ). PPRS Cronbach's alpha was 0.66 ; corrected item-total correlation, $0.14-0.55$; and item homogeneity, 0.23 . Factor analysis identified two factors $(58.5 \%$ of the variance). Low correlation coefficients were found between PPRS and SCOPA-Motor, MMSE, HADS, and disease severity $\left(r_{\mathrm{S}} \leq 0.30\right)$. PPRS scores significantly increased with disease duration, HY stage, and CISI-PD severity level ( $p<0.001)$. The SEM was 1.06. PPRS items were judged to be relevant relevant in $80.6 \%$ of the panel's evaluations. With the exception of item 2 (endorsed by only 4 experts) 5 of the 6 experts endorsed the complete scale and the individual items.

Conclusion: Overall, the results suggest that the PPRS is a useful tool for evaluation of psychosis in PD, although displays limitations in content validity.

\section{P14/62 \\ Epidemiology of Parkinson Disease in Moscow}

\author{
Bezdolniy Y., Katunina E., Gusev E., Avakyan G. \\ Russian State Medical University, Moscow, Russian \\ Federation
}

The aim of our research was to estimate clinical and epidemiological indexes of Parkinson disease (PD) in Moscow population.

Materials and Methods: The study was spent in neurological department of regional clinic of northeast Moscow district from 01.07.06 till 31.06.08. The population of northeast Moscow district is 1237899 people (12.4\% from Moscow population). Organization of neurological department work consists of information collection about PD cases from 22 clinics of northeast Moscow district, examination of patients for confirmation of PD and treatment. The analysis of database was performed.

Results. 947 cases of PD were analyzed. Prevalence of PD was 76.5/100,000. Age analysis represented male domination in all age groups with the greatest indexes older 70 years. General incidence of PD was 7.63/100,000 a year. Incidence of PD was $9.85 / 100,000$ for female a year , 5.18/100,000 for male a year. The incidence among female is more than male, but standardization of age structure population revealed domination of male incidence at the age of 60-69. The middle age of incidence of PD among male was $62.35 \pm 9.59$, among female $64.12 \pm 9.81$.

Cognitive disorders among patients with PD made 64\%: $34 \%$ from this sum - was dementia. Light cognitive disorders were marked in $30 \%$ cases, light and middle dementia in $31 \%$ cases. Hard dementia was fixed in $3 \%$ cases.

$71 \%$ of patients had depression disorders. Light and middle depression was marked in $32 \%$ cases. Strong and hard depression was revealed in $39 \%$ cases. There was no correlation between prevalence of depression and length of disease.

\section{P14/63 \\ Has Tremor Dominant Type of Parkinson's Disease a Better Prognosis or Not?}

Bokor M., Faragó A.

Municipal Hospital Nyírő Gyula, Neurology, Budapest, Hungary

Our aim was to investigate whether the prognosis of the tremor dominant type (TD) of Parkinson's disease (PD) is better or not compared to the other two subtypes of the disease.

A population based case control study was conducted with a follow up period of 18 years. In 1990, all PD patients were investigated in one district of Budapest (with 131.563 inhabitants). Among the 203 suspect cases 162 were diagnosed as PD by a specialist in the field of movement disorders. After having completed an interviewer-administered structured questionnaire - containing data about many tasks - the patients were followed for 6,12 and 18 years, and the mortality data were analyzed. 
The mean age of the TD patients was higher - 84,8(5.8) years - than rigid-akinetic (RA) patients - 81,2(5,7) years - and the equivalent (RAT) patients - 81,0(6,3) years. The mean duration of the illness in the TD type was longer - 14,5(7,7) years - than that of the RA - 10,3 $(7,7)$ years and the RAT patients - 11,2(11,1) years. The differences were not significant. After 6 years, the survival rates were the following: TD 70,4\%, RA 3,7 \%, RAT 25,9\% respectively. After 12 years, the survival rates were the following: TD $60,0 \%$, RA $0 \%$, RAT $40,0 \%$ respectively. By the end of the follow-up period 5 patients were alive in the last distribution.

The results showed a little better prognosis for patients of the TD group, compared to the other two subgroups - although the difference was not significant.

\section{P14/64}

\section{Validity and Reliability of the Persian Version of the Parkinson Disease Quality of Life (PDQL)}

\author{
Ghaem H. ${ }^{1}$, Borhani Haghighi A. ${ }^{2}$, Zeighami B. ${ }^{3}$, \\ Dehghan A. ${ }^{4}$ \\ 1Shiraz University of Medical Sciences, Shiraz, Iran, \\ Islamic Republic of Iran, ${ }^{2}$ Shiraz University of Medical \\ Sciences, Neurology, Shiraz, Iran, Islamic Republic of Iran, \\ ${ }^{3}$ Shiraz University of Medical Sciences, Epidemiology, \\ Shiraz, Iran, Islamic Republic of Iran, ${ }^{4}$ Health Center, Lar, \\ Iran, Islamic Republic of Iran
}

Introduction: To translate and test the reliability and validity of the Parkinson disease quality of life Questionnaire (PDQL) in Iranian patient with Parkinson disease.

Material Method: using a standard 'Forward-Backward' translation, cognitive debriefing and cultural adaptation procedure, the English version of PDQL translated to Persian which is the Iranian official language.

The subjects were patients with Parkinson referred to Motaharri clinic in Shiraz. Demographic data and Epidemiological data concerning disease stage, Quality of sleep, stage of disease were recorded..the reliability of the questionnaire was assessed by Coronbach's alpha coefficient. Construct validity was assessed through factor analysis. Convergent and Discriminant validity was assessed by sperman's correlation coefficient.

Results: patient with Parkinson (male: 67(62.03\%) and female $41(37 / 97 \%)$ ), with mean \pm SD age of $64 / 49 \pm 11 / 73$ Were enrolled in study. Coronbach`s alpha was 0.95 . Range of convergent validity was $0 / 42-0 / 83$ and range of Discriminate validity was 0/15-/70. Rang of Factorial analysis load was 0/119-0/801.

Conclusion: The Persian version of the PDQL Questionnaire has a good structure characteristic; it is a reliable and valid instrument and can be used for measuring the effect of Parkinson on the quality of life.

\section{P14/65 \\ Parkinson Disease Mortality in Japan, 1995- 2004: Analysis of Clustering}

\author{
Doi Y., Yokoyama T., Takahashi K., Tango T. \\ National Institute of Public Health, Wako, Japan
}

Background: Parkinson disease (PD) is of great concern as a social and economic burden in Japan's super-aging society. Our present study examined the geographical clustering of deaths from PD on a national basis in the past 10 years.

Methods: Data on underlying cause-of-death for PD were taken from the national death certificate database (coded as G20 for ICD-10). A total of 27,685 PD deaths (13,033 male and 14,652 female) during the period of 1995 to 2004 were used for analysis using empirical Bayes estimates of standardized mortality ratios and the flexible spatial scan statistic to detect clusters. To detect geographically-accumulated clusters, $\mathrm{P}$ values were obtained using Monte Carlo hypothesis testing (with $\mathrm{P}<0.05$ as statistical significance).

Results: After adjustment for age, four statistically significant clusters were detected from the central to western parts of the mainland for females: Osaka-Hyogo-Kyoto-Wakayama-Nara $(\mathrm{P}<0.01, \mathrm{RR}=1.30)$, Yamanashi-Nagano-Shizuoka $(\mathrm{P}<0.01$, $\mathrm{RR}=1.52)$, Tokyo $(\mathrm{P}<0.01, \mathrm{RR}=1.32)$, and Hyogo-ShimaneTottori-Okayama-Kyoto $(\mathrm{P}<0.05, \mathrm{RR}=1.29)$. For males, two more clusters were found in the northeast of the mainland and the southern tip of Kyushu island: Osaka-Hyogo-Kyoto-ShigaNara $(\mathrm{P}<0.01, \mathrm{RR}=1.21)$, Nagano-Yamanashi-Gifu $(\mathrm{P}<0.01$, $\mathrm{RR}=1.41)$, Tokyo-Kanagawa-Saitama $(\mathrm{P}<0.01, \mathrm{RR}=1.18)$, Shimane-Hiroshima-Yamaguchi-Okayama-Tottori $\quad(\mathrm{P}<0.05$, $R R=1.28)$, Sendai $(P<0.01, R R=1.66)$ and Kagoshima $(P<0.05$, $\mathrm{RR}=2.51$ ).

Conclusion: The present study identified several statistically significant clusters of deaths from PD mainly located in the mainland of Japan, and suggested some of them as candidate locations for future epidemiological studies on PD.

\section{P14/66 \\ Incidence of Focal Dystonia in Belarus}

Rushkevich Y., Likhachev S., Charnukha T.

National Neurology and Neurosurgery Recearch Center, Neurology, Minsk, Belarus

Background: To investigate focal dystonia (FD) epidemiological parameters in Belarus from 2005 to 2008.

Methods: We assembled the database of patients with FD $(n=459)$ in Belarus. We included into the database patients with diagnosed FD with age range from 18 up to 70 years permanently live in Belarus. Cases were verified by dystonia specialists, who performed them botulinum toxin treatment. Incidence parameters such as age of onset, male/female ratio were studied.

Results: total number of FD cases - 510 patients, among them $400(78,4 \%)$ - spasmodic torticollis (ST), $82(16,1 \%)$ - blefarospasmus and $28(5,5 \%)$ patients with other forms of FD. The 
incidence ratio of FD were: 2008 - 5, 27; 2007- 5,48; 2006 - 5,5; 2005- 5,3 per 100000 of population. ST is the most common form of FD and constituted near $80 \%$. The incidence of ST was in 2005 - 4,10; $2006-4,2 ; 2007-4,12$ and in $2008-4,13$ per 100000 of population. The male/female ratio among the patients with FD was 1:0,75.

Conclusion: No differences as among sex ratio as incidence parameters was found. The incidence of FD In Belarus is lesser in comparison with European countries (Warner et al., 2000). Possibly, it's connected with low specialized medical aid availability or difficulties with FD diagnosis.

\section{P15}

\section{Peripheral Neuropathy}

P15/67

Epidemiology and Characteristics of Guillain-Barré Syndrome in the Northwest of Iran

Yazdchi M. ${ }^{1}$, Arami M.A. ${ }^{2}$

${ }^{1}$ Tabriz University of Medical Sciences, Tabriz University Neuroscience Research Center, Tabriz, Iran, Islamic Republic of Iran, ${ }^{2}$ Milad General Hospital, Neurology, Tehran, Iran, Islamic Republic of Iran Barré

Background: Little is known about the incidence of Guillain-

syndrome (GBS) in Iran. We determined the incidence and evaluated prognostic factors for GBS in a prospective, population-based study.

Patients and Methods: We evaluated and followed all patients with a diagnosis of GBS admitted to three referral neurology centers in East Azerbaijan province over a 1-year period. Clinical and electrophysiological characteristics of cases were reviewed and analyzed.

Results: A total of 76 patients were found, corresponding to a crude annual incidence rate of $2.11 / 100000$ population. Six patients $(7.9 \%)$ died acutely within 21 days from the onset of the disease. Acute mortality was due to respiratory involvement, sepsis and acute autonomic system dysfunction. The persistence of disability after the acute phase was related to axonal involvement $(\mathrm{OR}=3.19,95 \% \mathrm{CI}, 1.65$ to 6.16$)$. There was a significant correlation between a history of diarrhea and a further need for mechanical ventilation $(\mathrm{P}<0.05)$. Mechanically ventilated patients had a low GBS disability score on discharge compared with patients not mechanically ventilated $(\mathrm{P}=0.05)$.

Conclusions: The incidence rates of GBS in our province is similar to that in other countries. Acute mortality in GBS was mostly due to poor respiratory care of patients and infective complications, but disability and probably late mortality were due to axonal nerve injury.

\section{P17 \\ Stroke and Other Cerebrovascular Disorders}

\section{P17/68 \\ Association Between Renal Function and Outcomes in Patients with Acute Stroke}

Hao Z., Liu M., Wu B., Yang W., Lin S., Wang D., Tao W., Kong F.

Department of Neurology, West China Hospital, Sichuan University, Chengdu, China

Background: Several studies suggested that renal dysfunction was an independent predictive factor of poor outcome in patients with myocardial infarction or coronary surgery. Few studies investigated influence of renal dysfunction on outcomes in acute stroke.

Methods: We examined the association between baseline renal function and one year outcomes in 1758 consecutive Chinese patients with acute stroke within 24 hours of symptom onset by using multivariate logistical regression model. The estimate glomerular filtration rate (eGFR) was calculated by the Modification of Diet in Renal Disease equation. Renal dysfunction was defined as $\mathrm{eGFR}<60 \mathrm{~mL} / \mathrm{min} / 1.73 \mathrm{~m}^{2}$. We followed up patients at $12 \mathrm{month}$ after stroke for outcomes (death, disability).

Results: The distribution of eGFR on admission was similar to normal distribution and the mean was $75.87 \pm 38.31$. Of the 1758 cases, 463 cases had decreased eGFR, which accounted for $26.3 \%$. The eGFR on admission was the independent prognostic factor for death/disability at 12 th month, but not for death at 12 th month. The patients with renal dysfunction had a significant higher risk of death/disability than those with eGFR $>90 \mathrm{~mL} /$ $\mathrm{min} / 1.73 \mathrm{~m}^{2}(\mathrm{OR}=1.864,95 \% \mathrm{CI}, 1.170-2.970)$. In addition to renal function, NIHSS at admission and age were independent prognostic factors of death/disability at the end of 12 th month.

Conclusions: Our study indicated that more than $1 / 4$ of acute stroke patients had Renal dysfunction. Renal dysfunction measured by eGFR is a independent predictive factor for one year outcomes in acute stroke patients.

Poster Presentations 


\section{P17/69 \\ Profiles of Acute CVD Patients in A/E Department of a Hospital in Jakarta, Indonesia January-June 2005}

Wreksoatmodjo $B$.

Atmajaya Catholic University, Neurology, Jakarta Utara, Indonesia

Background: Cerebrovascular disease (CVD) is one of the most frequent neurological diseases. CVD often presents as acute attack and seen in accident/emergency (A/E) department; the earlier the diagnosis and management, the better the prognosis. We want to survey the incidence of CVD and the use of CT scan as early diagnostic tool.

Methods: Survey in a hospital in Jakarta during 6-month period (January-June 2005)

Results: During study period, 97 cases were registered comprising $1.24 \%$ of all cases in $\mathrm{A} / \mathrm{E}$ department; $80.41 \%$ are $\geq 50$ years, with equal proportion of gender/sex. The type of most cases (50 - 64.10\%) was not clinically diagnosed until brain CT scan was done; but this may be due to the 24-hour availability of CT scan facility, so diagnosis was deferred until the result of CT scan was available.Non hemorrhagic type was more common (48 vs. 30 cases); this is in accordance with earlier reports. Subarachnoid hemorrhage was diagnosed in only 5 cases. Three cases formerly suspected as CVD were finally diagnosed as brain tumor (2 cases) and subdural hematoma (1 case).

Conclusion: CVD cases was mostly non-hemorrhagic type. Other cases that mimic CVD should serve as a reminder that there are clinically similar other diseases, and consequently brain $\mathrm{CT}$ scan should serve as an important diagnostic tool.

P17/70

\section{Netrin-1 Improves the Effect of Mesenchymal Stem Cells in Angiogenesis}

\section{Ding $X$ \\ Nanjing Medical University, Neurology, Nanjing, China}

We examined the impacts of application of netrin-1 protein and mesenchymal stem cell (MSC) implantation on therapeutic angiogenesis in a rat model of hind limb ischemia. Co-injection of MSCs with a recombinant netrin-1 protein markedly augmented the angiographic score and capillary density, improved function of the ischemic limb and increased levels of vascular endothelial growth factor in the plasma and damaged tissues. Further studies suggested that netrin-1 promoted MSC migration and enhanced its ability to participate in tube formation. These results demonstrated that transplantation of MSCs by co-application with netrin-1 protein significantly could improve therapeutic effect of MSCs, hence may provide a novel therapeutic approach for ischemic disease

\section{$\mathrm{P} 17 / 71$ \\ Transition in Stroke Incidence Between 1985 and 2005 in Akita, Japan}

\author{
Suzuki K. ${ }^{1}$, Nagata K. ${ }^{2}$, Izumi M. ${ }^{3}$ \\ ${ }^{1}$ Research Institute for Brain and Blood Vessels, \\ Epidemiology, Akita, Japan, ${ }^{2}$ Research Institute for Brain \\ and Blood Vessels, Neurology, Akita, Japan, ${ }^{3}$ Research \\ Institute for Brain and Blood Vessels, Cardiology, Akita, \\ Japan
}

Background: Akita stroke onset registry (ASOR) has a long history since 1973 and is now the biggest epidemiological stroke registry in Japan. The ASOR is targeting 1.2 million cases, that are equivalent to the whole population of Akita prefecture. Based on the ASOR data, we analyzed the transition of the stroke incidence between 1985 and 2005.

Methods: Prefecture-wide stroke registry covered 67276 cases between 1985 and 2005, and 53921 cases of first-ever stroke cases was used for the analysis. The number of cases, and ageadjusted incidence were compared by subtypes in every 5 years from 1985.

Results: Although the total number of stroke cases increased from 892 to 3133 between 1985 and 2005, the age-adjusted stroke incidence was approximately $160 / 100,000$ in men and $100 / 100,000$ in women throughout the observation period. This can be explained by the aging effect, because the senior population has been increasing in Akita prefecture. These figures suggest that the stroke incidence was never improved in last two decades. A similar trend was observed in the incidence of stroke subtypes. There was no significant change in the means blood pressure among the population.

Conclusion: There was no significant change in the ageadjusted stroke incidence and the main risk factors in the last two decades in Japan. As the hypertension is regarded as a main risk factor of stroke, management of high blood pressure among the population will be the best strategy for preventing stroke in all subtypes.

\section{P17/72}

\section{Stroke Severity and Three-Year Mortality}

Suto Y. ${ }^{1}$, Kowa H. ${ }^{1}$, Nakayasu H. ${ }^{2}$, Nakashima K. ${ }^{1}$

${ }^{1}$ Department of Neurology, Institute of Neurological Sciences, Faculty of Medicine, Tottori University, Yonago, Japan, ${ }^{2}$ Department of Neurology, Tottori Prefectural Central Hospital, Tottori, Japan

The present study estimates mortality rates in patients with ischemic stroke by comparing the ability to perform routine daily activities after discharge from acute care hospitals. Patients with acute ischemic stroke were registered between December 1999 and November 2003. Nine hundred and two patients (mean age $72.2 \pm 11.6$ ) with a first-ever ischemic stroke were followed up for a mean period of $771.0 \pm 415.6$ days. Mortality was assessed from the viewpoint of the routine daily activities when they left an 
acute care hospital. Data were statistically analyzed using KaplanMeyer estimates, and hazard ratios were analyzed using the Cox proportional hazard model. Mortality rates were $8.4 \%$ in walking independently, $10.4 \%$ in walking with support, $30.2 \%$ in using a wheelchair, and $58.4 \%$ in moving with a stretcher. Prognostic factors for increased mortality were advanced age and male gender. After adjusting for these factors, the hazard ratios of poor survival compared with patients who could walk independently were $1.16(\mathrm{p}=0.59)$ for those who could walk with support, $3.61(\mathrm{p}<$ $0.05)$ for those who were wheelchair-bound and $4.35(\mathrm{p}<0.05)$ for those who moved with a stretcher, respectively. Mortality rates are high among patients with severe stroke.

\section{$\mathrm{P} 17 / 73$ \\ Long Term Mortality in Persons Suffering from Stroke. Data from the NEDICES Cohort Study}

Bermejo- Pareja F.1, Rodriguez Rodriguez C. ${ }^{1}$, Trincado R. ${ }^{2}$, Benito-Leon J. ${ }^{1}$, Villarejo A. ${ }^{2}$, Boix R. ${ }^{3}$, Puertas-Martin V. ${ }^{2}$, Medrano M. ${ }^{4}$, Vega S. ${ }^{5}$, Diaz- Guzman J. ${ }^{2}$, For Neurologica I Disorders in Central Spain (NEDICES) Study Group

${ }^{1}$ UH 12 de Octubre and Ciber Neurodegenerative Disorders, Neurology, Madrid, Spain, 2UH 12 de Octubre, Neurology, Madrid, Spain, ${ }^{3}$ Instituto de Salud Carlos III, Epidemiology, Madrid, Spain, ${ }^{4}$ Carlos III Institute of Health, Epidemiology, Madrid, Spain, ${ }^{5}$ Espinar Health Center, El Espinar, Spain

Objective: To evaluate the long term mortality (5 and 10 years) after the baseline wave (1994) of stroke participants in the Neurological Disorders in Central Spain (NEDICES) Cohort Study.

Background: The NEDICES Study is a population-based cohort study in the elderly (65 years and more) with 5, 278 screened participants at baseline. The mortality of this cohort has been evaluated by means of the National Death Registry of Spain from 1994 to 2004.

Methods: At baseline, diagnosis of stroke was performed by neurologists. The mortality risk was analysed with Cox proportional hazards model with time-dependent variable for age, adjustments for potential covariates such as educational level and co-morbidity. Survival study was performed with Kaplan-Meier analysis.

Results: The mortality at ten years in the 5,024 participants without stroke was $33.9 \%$ (1,705 persons). In 254 stroke participants it was $52.9 \%$ (136 persons). When adjusted for sociodemographic variables and co-morbidity in the Cox proportional hazards model, suffering from stroke was associated with an increased risk of mortality in males (HR $=1.47$; CI 95\%: 1.11 $1.92)$ but not in females at 10 years. Using the Kaplan-Meier method, mean survival was 8.71 years (CI 95\%: 8.61 - 8.80) for participants without stroke and 6.94 years $(6.45-7.43)$ for stroke participants. At 5 years, mortality analyses were performed.
Conclusions: In stroke patients long term mortality is clearly increased in men but not in women in the NEDICES cohort (at ten years after baseline wave).

\section{P17/74 \\ Community-Based Study of Stroke Incidence: The Care-Cerebrovascular Aosta Registry Methodology}

\author{
Reggiani M., Bottacchi E., Corso G., Di Giovanni M., \\ Giardini $G$. \\ Ospedale Regionale, Stroke Unit, Department of \\ Neurology, Aosta, Italy
}

Background: Accurate measurement of stroke incidence is difficult and can be attempted only by community based studies. Here we report methodology of ascertainment of stroke cases in Aosta Valley (AV), Italy.

Methods: The AV is a Region in the North-West of Italy with only one main town Aosta (1/3 population) and only one Hospital with one ER. Ascertainment of cases was conducted from $1^{\text {st }}$ January 2004 to $31^{\text {st }}$ December 2008 and based on 5 sources: 1) daily review of ER admissions; 2) GP referrals; 3) neurological out-patient clinic; 4) review of hospital diagnostic codes (430 to 438 ICD9-CM); 5) review of mortality list. Notification was collected on a form. A clinical panel of stroke experts reviewed all notified cases diagnosis. Confirmed strokes were collected in a digital database, which is at the moment complete for a total of 1366 patients (data inclusion for the second 2008 semester is on going).

Results: During five years of study a diagnosis of stroke was supposed for 1830 patients, of these 195 were not residents in Aosta valley and therefore excluded. For the remaining 1635 patients a diagnosis of incident stroke was confirmed by the clinical panel for 1366 . Notification sources distribution was the following: 1) notificated 978, confirmed 788 (81\%); 2) notificated 426 , confirmed $376(88 \%)$; 3 ) notificated 71 , confirmed $62(87 \%)$; 4) notificated 138, confirmed $118(86 \%)$; 5) 22 confirmed cases.

Conclusion: Our analysis suggests the need for broad-based strategies of ascertainment to obtain reliable stroke incidence data. 


\section{$\mathrm{P} 17 / 75$}

\section{Hospital Arrival Time After Onset of Stroke and Associated Factors in a Japanese Population: Takashima Stroke Registry 1988-2002}

Kita Y. ${ }^{1}$, Turin $T^{1}{ }^{1}$, Rumana ${ }^{1}{ }^{1}$, Nakamura $Y^{2}{ }^{2}$, Takashima ${ }^{1}{ }^{1}$, lchikawa M. ${ }^{3}$, Sugihara $H^{3}{ }^{3}$, Morita $Y^{4}$, Hirose $\mathrm{K}^{5}$,

Okayama A. ${ }^{6}$, Miura K. ${ }^{1}$, Ueshima H. ${ }^{1}$

${ }^{1}$ Department of Health Science, Shiga University of Medical Science, Otsu City, Japan, ${ }^{2}$ Kyoto Women's University, Kyoto, Japan, ${ }^{3} T a k a s h i m a$ General Hospital, Takashima, Shiga, Japan, ${ }^{4}$ Makino Hospital, Takashima, Shiga, Japan, ${ }^{5}$ Otsu Red Cross Hospital, Otsu City, Shiga, Japan, ${ }^{6}$ The First Inst. for Health Promotion and Health Care, Tokyo, Japan

Objective: Successful acute stroke intervention depends on early hospitalization Time interval between stroke onsets to hospitalization was examined to identify the factors influencing the interval and also to determine whether treatment time window expansion will translate into more treatment.

Methods: Data was obtained from Takashima Stroke Registry covering approximately 55,000 residents in central Japan. During 1988-2002, the interval between stroke onset to hospitalization was available for 1446 registered first-ever stroke patients (men:761 and women:685). Multivariate regression analyses were performed to evaluate the factors influencing early and late admission.

Results: The proportions of the patients who arrived within 3 hours of onset were $61.4 \%$. Only $8.7 \%$ of the patients arrived during the 3-6 hours interval. Substantial (29.9\%) patients reached after 6 hours. For moderate and severe cases, $67.7 \%$ and $81.4 \%$ patients arrived within $\leq 3$ hours, whereas only $45.1 \%$ of mildly effected patients presented early. Early stroke hospitalization was significantly associated with presence of focal neurological deficit during onset, severity of the stroke event, and time of stroke onset. On the other hand presence of drinking habit was related to delayed hospitalization.

Conclusion: Clinically more severe stroke patients were hospitalized earlier and nocturnal strokes had delayed admission. Even expanding the therapeutic time window from $\leq 3$ to $\leq 6$ hours, there are possibilities that a substantial proportion of patients would stay out of the benefit of acute intervention. These results stress the need for health promotion intervention focusing on immediate hospitalization for acute stroke events.

\section{P17/76 \\ Optimal Intensity of International Normalized Ratio in Warfarin Therapy for Primary Prevention of Stroke in Chinese Patients with Atrial Fibrillation}

Chiang C.-E.

Taipei Veterans General Hospital/National Yang-Ming University, Division of Cardiology, Department of Medicine, Taipei, Taiwan, Republic of China

Background: The optimal intensity of warfarin therapy for primary prevention in non-valvular atrial fibrillation (AF) in western countries is within 2.0-3.0 of international normalized ratio (INR). The optimal INR ratio for Chinese remains unclear.

Methods: The study consisted of 394 patients with a CHADS2 score $\geq 2$. They were followed up for 2 years and were divided into 4 groups according to the INR reached: $\leq 1.49$ (group 1), 1.50-1.99 (group 2), 2.00-2.59 (group 3), $\geq 2.60$ (group 4). The incidence of intra-cranial hemorrhage, all hemorrhage, and TE was compared in the 4 groups.

Results: There were 36 patients in group 1 (9.1\%), 210 in group $2(53.3 \%), 110$ in group $3(27.9 \%)$, and 38 in group 4 $(9.6 \%)$. Intra-cranial hemorrhage occurred in 0 patient in group 1,0 in group 2, 2 in group $3(3.6 \%, \mathrm{P}<0.05$ vs group 1 and group $2)$, and 3 in group $4(7.9 \%, P<0.01$ vs group 1 and group 2$)$. All bleeding occurred in 0 patient in group 1,3 in group $2(1.4 \%)$, 16 in group $3(14.5 \%, \mathrm{P}<0.01$ vs group 1 and group 2$)$, and 9 in group $4(23.7 \%, \mathrm{P}<0.001$ vs group 1 and group 2$)$. TE occurred in 1 patient in group $1(2.8 \%), 3$ in group $2(1.4 \%), 2$ in group 3 $(1.8 \%)$, and 0 in group 4 (all $\mathrm{P}>0.05$ ).

Conclusions: The optimal INR for Chinese patients with AF is 1.50-1.99 in this study. Large-scale prospective study is needed to confirm our findings.

P17/77

\section{High Incidence of Ischemic Stroke Is Responsible for the Higher Incidence of All Strokes in Hungary}

Óváry C. ${ }^{1}$, Szabó G. ${ }^{2}$, Nagy Z. ${ }^{3}$

${ }^{1}$ National Institute of Neuroscience, Budapest, Hungary,

${ }^{2}$ Kenessey Albert Hospital, Balassagyarmat, Hungary,

${ }^{3}$ Semmelweis Medical University, Budapest, Hungary

Background: The incidence of stroke in Hungary dramatically exceeds that of in the Western European countries. The aim of our study was to compare subtype-specific stroke incidence rates to analyze what drives the variation.

Methods: We analyzed our standardized, prospective stroke register in 11 stroke centers with a catchment area of 1.5 million during 18 months. Crude and age-standardized incidence rates were calculated for 6667 first strokes. The denominators for the calculation of the incidence rates were the 1991 census data. 
Subtype-specific incidence rates were also calculated and standardized to the European population.

Results: The age-standardized incidence of ischemic stroke (200.3/ 100 000/year) was about twice of the incidence published in Western European stroke registries, meanwhile the incidence of intracerebral hemorrhage (11.9/100 000/year) was similar.

Conclusion: High stroke incidence in Hungary, and consequently higher stroke mortality, is due to the very high incidence of ischemic strokes. Further investigations should focus on the prevalence of vascular risk factors in our population to understand the variation.

$\mathrm{P} 17 / 78$

\section{Antiplatelet Therapy is Associated with a Lower Risk of Death and Recurrent Cerebrovascular Events After Ischemic Stroke}

Ding D., Lu C.-Z., Fu J.-H., Hong Z., The China Ischemic Str oke Registry Study (C/SRS) Investigators

Institute of Neurology, Fudan University, Shanghai, China

Background: Evidence of the beneficial effects of antiplatelet therapy after ischemic stroke is currently lacking in China. This multi-center, prospective study aimed to provide data using a large sample size to gain insights into the association between antiplatelet therapy and the decrease risk of death and recurrent cerebrovascular event after ischemic stroke in Chinese patients.

Methods: We enrolled 1951 ischemic stroke patients. Demographic data, pre-stroke risk factors, severity of neurological deficits, and disability graded on National Institutes of Health Stroke Scale (NIHSS) and Modified Rankin Scale (MRS) of each patient were measured and recorded. Regular follow-ups by interview were performed for 12 months post-recruitment. Clinical end-points were all-cause death and recurrence of cerebrovascular event.

Results: The all-cause mortality was 1.88 per 100 personyears during the follow-up period. Recurrent fatal and nonfatal cerebrovascular events were detected in 90 patients, accounting for 4.24 per 100 person-years of cumulative incidence. After adjustment by other variables, antiplatelet therapy was identified as the independent protective predictor for all-cause death (HR, $0.42 ; 95 \% \mathrm{CI}, 0.21-0.86 ; \mathrm{P}=0.017)$ and recurrent cerebrovascular event (HR, 0.58; 95\% CI, 0.36-0.92; P = 0.021). Among the survival cases, antiplatelet therapy was also the independent predictor for improvement in NIHSS (HR, 1.27; 95\% CI, 1.07-1.51; $\mathrm{P}=0.006)$ and $\mathrm{MRS}(\mathrm{HR}, 1.25 ; 95 \% \mathrm{CI}, 1.02-1.52 ; \mathrm{P}=0.031)$ scores.

Conclusions: The data from our population is consistent with the benefit expected from antiplatelet therapy demonstrated in other populations.

\section{P17/79}

\section{The Study of the Association Between Angiotensinogen Gene T704C, C521T Polymorphism and Cerebral Infarction}

\author{
Sun H.Y., He J.L., Yang G.A., Wu L.E. \\ The First Affiliated Hospital of Baotou Medical College, \\ Baotou, China
}

Objective: This study is intended to study the relationship between the AGT gene T704C, C521T mononucleotide polymorphism and cerebral infarction.

Methods: A case-control study of 82 cases and 89 controls was carried out with polymerase chain reaction-restriction fragment length polymorphism (PCR-RFLP), the AGT gene 704 and 521 sites were tested. The study compared and analyzed the differences of the distribution rate of genetype and the allelic as well as analying the effect the genetic factor has on cerebral infarction. Independent fatalness toward cerebral infarction were analyzed by Logistic regression. Meanwhile, by observing the objectives' haplotype structure, polymorphism combination related to cerebral infarction was expected to be found.

Results: In the cerebral infarction group, AGT 704 site CC genotypic frequency $63.4 \%$ and $\mathrm{C}$ allele frequency $79.9 \%$ were significantly higher than the control group $34.8 \%$ and $61.2 \%(\mathrm{P}<$ $0.05)$; AGT gene 521 site TT genotypic frequency $22.0 \%$ and T allele frequency $28.0 \%$ were significantly higher than the control group6.7\%, and11.8\% $(\mathrm{P}<0.05)$; By multiple factor Logistic regression analysis, AGT 704 CC genetype, AGT 521 TT genetype could increase the rate of the cerebral infarction, pathogenetic relative risk rates(OR) were respectively3.667, 3.492;4. The distribution rate of the haplotype 521T-704C was significantly higher than the control group $(\mathrm{P}=0.001)$.

Conclusion: 1 . AGT gene 704CC genetype and $\mathrm{C}$ allele might be susceptible factors. 2. AGT gene 521 TT genetype and T allele might be susceptible factors.3. haplotype 521T - 704C might be the heredity risk factor of the development of cerebral infarction.

P17/80

\section{Analysis of the Seasonal Variability in Spontaneous Cervical Artery Dissection}

Evers S., Milz R.U., Akova-Öztürk E., Ringelstein E.-B.

University of Muenster, Neurology, Muenster, Germany

There is an evidence for seasonal variability in spontanous cervical artery dissection (SCAD) with a significant peak in wintertime

Methods: We analysed the seasonal variability of 223 Patients between 16 and 72 Years with sCAD. 155 of them had consecutive ischaemic stroke. Vascular risk factors were also recorded. We analysed the seasonality of all patients and compared the seasonal variability of patients with arterial hypertension to these without arterial hypertension.

Results: We found a significant seasonal peak of sCAD in winter (November 1st until January 31st). The highest number of 
sCAD was found in January. The analysis of patients with arterial hypertension was significant $(\mathrm{p}=0.022)$. The analysis of patients without arterial hypertension was not. Other vascular risk factors, such as cigarette smoking, diabetes mellitus hyperlipidaemia, investigated in our study failed to show any significant influence on the seasonal variability of sCAD.

Conclusions: We conclude that SCAD shows a seasonal variability with a peak in wintertime and that the only conventional risk factor associated with this observation is arterial hypertension. There might be a pathophysiological connection between hypertension and SCAD. Temperature- and air pressure-related changes in blood-pressure, coagulation parameters, rheological factors, physical activity, diet, and air pollution have been considered as the underlying mechanism. Further studies are warranted to clarify these issues.

\section{P17/82 \\ Conventional Vascular Risk Factors in Spontaneous Cervical Artery Dissection: A Case-Control Study}

\author{
Evers S., Milz R.U., Akova-Öztürk E., Ringelstein E.-B.
}

University of Muenster, Neurology, Muenster, Germany

Background: Although the aetiology of spontaneous cervical artery dissection remains unclear, several constitutional and environmental risk factors have been discovered to be associated with spontaneous carotid or vertebral artery dissection (SCAD and sVAD). However, conventional vascular risk factors in patients with SCAD or SVAD have not been studied in a case-control design.

Methods: We analysed the incidence of conventional vascular risk factors in patients with SCAD and sVAD. The data were compared in a case-control design to 358 sex and age matched healthy control subjects.

Results: The analysis of all patients compared to the healthy control group showed arterial hypertension $(p<0.001)$, smoking $(\mathrm{p}=0.039)$, hyperlipidaemia $(\mathrm{p}<0.001)$, and fibromuscular dysplasia $(p<0.001)$ to be significantly more prevalent in patients with SCAD or sVAD. The occurrence of venous disease in patients themselves $(p<0.001)$ or in any first degree relative $(p<0.001)$ and the use of oral contraceptives $(p=0.002)$ turned out to be significantly more prevalent in the healthy control group. All subgroup analyses showed similar results.

Conclusions: We found arterial hypertension, smoking, hyperlipidaemia, and fibromuscular dysplasia to be significant risk factors for sCAD and sVAD. All subgroup analyses showed similar results suggesting that these risk factors are relevant for the development of SCAD or SVAD and not for the infarction. Venous diseases in patients themselves or in their family members are possibly protective factors for sCAD or sVAD. Oral contraceptive use also seems to be protective.

\section{P17/83}

Gender Comparisons of Subarachnoid Hemorrhage Incidence in Northern Greece: The Evros Registry of Subarachnoid Hemorrhage (Evro-SAH)

\author{
Vadikolias K. ', Tsivgoulis G. ${ }^{1}$, Heliopoulos I. ${ }^{1}$, \\ Papaioakim M. ${ }^{2}$, Aggelopoulou C. ${ }^{1}$, Serdari A. ${ }^{1}$, Birbilis T. ${ }^{3}$, \\ Piperidou C. $^{1}$ \\ ${ }^{1}$ Department of Neurology, Democrition University of \\ Thrace, Alexandroupolis, Greece, ${ }^{2}$ Department of \\ Emergency Medicine, Democrition University of Thrace, \\ Alexandroupolis, Greece, ${ }^{3}$ Department of Neurosurgery, \\ Democrition University of Thrace, Alexandroupolis, \\ Greece
}

Background: Epidemiological studies have shown discrepant results concerning potential gender disparities in the incidence of subarachnoid hemorrhage (SAH) across different countries. Epidemiological data on subarachnoid hemorrhage (SAH) incidence and case fatality rates are scarce in the SouthEastern Mediterranean region. We conducted a population-based study in Evros province, located in north eastern Greece, to SAH incidence over a 5-year period and compared incidence and case fatality rates (CFR) between genders.

Methods: Evros province has a well-defined, largely-homogeneous population with healthcare organized around a single tertiary-care University Hospital. We organized a prospective computerized registry of permanent Evros residents admitted or transferred to our hospital with a diagnosis of SAH. Standard WHO definitions and overlapping case-finding methods were used to identify all cases of first-ever in a lifetime SAH in all age-groups, occurring during the study period. The diagnosis was confirmed by CT-scan in all hospitalized cases. Sudden deaths attributable to SAH were systematically recorded province-wide by our Forensic Department.

Results: During the 5-year period 51 cases of SAH were recorded (28 men/23 women). The crude annual incidencerates were 8.3/100.000persons (95\%CI:5.5-12.0) for men and 7.5/100.000 (95\%CI:4.8-11.3) for women. The standardized incidence rates for groups aged 45-84 years to the European population were 9.3/100.000 (5.8\%-12.8\%) for men and 6.5/100.000 (3.7\%-9.4\%) for women. The 28-day CFR was 36\% (21\%-54\%) for men and $35 \%(19 \%-55 \%)$ for women. No differences were noted in CFR $(p=0.945)$ and incidence $(p=0.611)$ between men and women.

Conclusions: No gender-differences in SAH incidence and CFR were documented in North-Eastern Greece. 


\section{P17/84}

\section{Seizures and EEG Patterns in the Acute Stroke Phase}

Correnti A. ${ }^{1}$, Dispenza S. ${ }^{2}$, Puca E. ${ }^{3}$, Lorenzano S. ${ }^{1}$, Toni D. ${ }^{3}$, Mecarelli O. ${ }^{2}$, Prencipe M. $^{3}$

1'Sapienza'-Università di Roma, Scienze Neurologiche, Roma, Italy, ${ }^{2}$ Sapienza'-Università di Roma,

Neurofisiopatologia, Roma, Italy, ${ }^{3 '}$ Sapienza'-Università di

Roma, Roma, Italy

Background: Stroke is the most common cause of acute symptomatic seizures in the elderly. Aim of this study was to analyze the occurrence of early epileptic seizures and status epilecticus (SE) in patients with stroke and correlate them with risk factors, stroke subtypes and mortality. We also analyzed the patterns of EEG.

Methods: We studied 232 stroke patients admitted to our stroke unit. EEG tracings were recorded within 24 hours after stroke.

Results: Overall, 125 patients were women (53.8\%) and 107 men $(46.2 \%)$ with a mean ( \pm S.D.) age of 71 years $( \pm 12)$. Hemorrhagic stroke was diagnosed in $55(23.3 \%)$ patients and ischemic stroke in $177(76.7 \%)$. The location of stroke was cortical in 117 patients $(50.4 \%)$, subcortical in $78(33,6 \%)$ and cortico-subcortical in 37 cases $(20 \%)$. Early sporadic seizures or SE were reported in $15(6.5 \%)$ patients, all occurring within 24 hours of stroke onset. Seizures were detected in $4(7.3 \%)$ patients with hemorrhagic stroke and in $11(6.2 \%)$ with ischemic stroke. EEG tracings showed focal or diffuse slowings of background activity in 195 patients (84\%), epileptiform sporadic abnormalities in 23 (10\%), Periodic Lateralized Epileptiform Discharges (PLEDs) in 14 patients $(6 \%)$. Mortality is not different between patients with seizures and those without.

Discussion: Our data confirm that in acute stroke phase early epileptic seizures are relatively rare and mainly represented by SE. The better described patterns are PLEDs, probably underestimated. Hence, EEG monitoring would be helpful to study this transient abnormality and to quantify its impact on prognosis.

\section{P17/85 \\ Pathogenetic Value of Nitric Oxide (NO) at Ischemic Stroke on a Background of Treatment with Gliatilin}

\section{Madjidova Y., Rasulova K. \\ Tashkent Medical Academy, Neurology, Tashkent, Uzbekistan}

Background: It is known, that NO synthesis is protective reaction that may comprehend all organism to what detection of inducible isoform of NO generation practically in all organs and cells testifies. The purpose of our research was to study pathogenetical mechanisms of NO-system at ischemic stroke (II) in patients with II admitted on acute period depending on stroke pathogenetical heterogeneity.
Methods: 30 patients with II aged 45 to 70 years were examined. NO metabolites levels were investigated in blood serum, using Griss reactant. Blood for research was taken from ulnar veins in quantity 3-5 $\mathrm{ml}$. For allocation of all total metabolites a nitrite-ion restored with the help of metabolic cadmium, impregnated with copper, up to a nitrite-ion. Patients took a natural membrane-stabilizator Gliatilin, choline alfoscerate. The data of 14 patients with discirculatory encephalopathy (DE) were the control group.

Results: Patients with DE had NO metabolites average levels $0.903 \pm 0.042 \mathrm{mcmol} / \mathrm{ml}$. At II and expressed hypoxemia NO levels were authentically increased in comparison with the control (up to $5.02 \pm 0.30 \mathrm{mcmol} / \mathrm{ml} ; \mathrm{P}<0.05$ ). Administration of Gliatilin reduced hypoxemia, and NO levels were authentically reduced, but nevertheless remained above control values. The marked changes in NO metabolites levels in blood at II can be considered as reflection of hypoxic stress, which treated with Gliatilin.

\section{P17/86}

\section{Temperature and Stroke Incidence}

Peters A. ${ }^{1}$, Radon K. ${ }^{2}$, Schenkel J. ${ }^{3}$, Hajat S. ${ }^{4}$

'University Hospital, Ludwig-Maximilians-University, Institute and Outpatient Clinic for Occupational, Social and Environmental Medicine, Department of Neurology, Campus Grosshadern, Munich, Germany, ${ }^{2}$ University Hospital, Ludwig-Maximilians-University, Institute and Outpatient Clinic for Occupational, Social and Environmental Medicine, Munich University Hospital, Munich, Germany, ${ }^{3}$ Department of Neurology, Neurological Rehabilitation, Neurophysiology and Stroke Unit, Community Hospital Munich-Harlaching, Munich, Germany, ${ }^{4}$ London School of Hygiene and Tropical Medicine, Public and Environmental Health Research Unit, London, United Kingdom

Background: Studies resulting from the heat wave 2003 examined the association between temperature and change in the incidence of cardiovascular events. As similar pathophysiological pathways could also lead to cerebrovascular strokes (CS), we aimed to assess the risk of CSs with regard to temperature in Southern Germany.

Methods: Time series analyses for stroke frequencies were performed for Munich (M) with regard to the local temperature $\left(^{\circ}\right.$ Celsius), additionally we adjusted for particular matter (PM10) as a marker for air pollution.

The dataset included 2808 stroke patients in 8 years (2000 2007 ) with admission to one community hospital. Only cases classified as stroke or transient ischaemic attack according to ICD10 criteria on discharge were taken for further analyses. Different time lags were evaluated to discuss prolonged pathophysiological mechanisms that could lead to cerebral ischaemia.

Results: Cold temperature showed to increase the risk for cerebrovascular events in both areas. Every one degree Celsius drop in temperature below a value of zero degrees lead to an increased risk for cerebrovascular events by $2.70 \%$ (95\% confidence interval $-0.73 ; 6.25)$. 
Conclusion: In contrast to the analyses performed during the heat wave 2003 with increased hospital admissions due to cardiovascular events, our dataset revealed elevated CEs during cold temperature periods in Germany. Increased arterial pressure, blood viscosity and red cell counts resulting from exposure to cold temperatures might lead to this increased stroke incidence. Due to the small case count further analyses with larger datasets are required.

\section{P17/87 \\ The Effect of Acupuncture on MBP mRNA Expression in Ischemic Stroke Rats}

\section{Duan J. ${ }^{1}$, Liu M. ${ }^{2}$}

${ }^{1}$ Clinic, Neurology Medicine, Tianjin, China, ${ }^{2}$ Clinic, Neurology Medicine, Chengdu, China

To observe neuroprotective effect of acupuncture on injured cerebral myelin in ischemic stroke rats from the expression of myelin basic protein (MBP) mRNA.

Methods: Adult male Sprague-Dawley rats were employed in the study, which were divided into normal, ischemia-reperfusion model, early acupuncture and late acupuncture groups by random digits table. Focal cerebral ischemia-reperfusion models were established with thread embolism method. Acpuncture was applied on different time after ischemia. The expression of MBP mRNA were observed continuously by using the methods of RT-PCR and Real-time fluorescent quantitative PCR (FQ-PCR) in all groups before operation and on different time after operation respectively.

\section{Results:}

1. In model group the expression of ischemic focus MBP mRNA rose gradually, the data on 7th day was significant $(\mathrm{P}<$ 0.05 ) by RT-PCR and FQ-PCR methods.

2. Compared with model group, the expression of ischemic focus MBP mRNA of acupuncture group rose rapidly on different time $(\mathrm{P}<0.05)$.

3. Compared with late acupuncture group, the expression of ischemic focus MBP mRNA rose higher and faster in the early acupuncture group $(\mathrm{P}<0.05)$.

Conclusion: The acupuncture treatment could promote remyelination by stimulating transcription of MBP mRNA obviously and increasing the synthesis of MBP mRNA of the ischemic focus after focal cerebral ischemia. Early acupuncture treatment more contribute to stimulating transcription of MBP mRNA

\section{P17/88}

The Study of the Association Between Renin Gene, Angiotensinogen Gene Polymorphism and Cerebral Infarction

\author{
He J.L., Sun H.Y., Yang G.A.
}

The First Affiliated Hospital of Baotou Medical College, Baotou, China

Objective: This study is intended to study the relationship between the REN gene G10631A, AGT gene C521T mononucleotide polymorphism and cerebral infarction.

Methods: A case-control study of 82 cases and 89 controls was carried out with polymerase chain reaction-restriction fragment length polymorphism (PCR-RFLP), and the REN gene 10631 site, AGT gene site were tested. The study compared and analyzed the differences of the distribution rate of genetype and the allelic as well as analying the effect the genetic factor has on cerebral infarction.

Results: 1 . In the cerebral infarction group, REN 10631 site AA genotypic frequency $31.7 \%$ and A allele frequency $49.4 \%$ were significantly higher than the control group $10.1 \%$ and $30.3 \%$ $(\mathrm{P}<0.05)$; AGT gene 521 site TT genotypic frequency $22.0 \%$ and $\mathrm{T}$ allele frequency $28.0 \%$ were significantly higher than the control group 6.7\%, and $11.8 \%(\mathrm{P}<0.05)$; By multiple factor Logistic regression analysis, REN 10631 AA genetype, AGT 521 TT genetype could increase the rate of the cerebral infarction, pathogenetic relative risk rates(OR) were respectively 2.798,3.492; The distribution rate of the haplotype 521T-10631A was significantly higher than the control group $(\mathrm{P}=0.001)$.

\section{Conclusion:}

1. REN gene 10631AA genetype and A allele might be susceptible factors.

2. AGT gene 521 TT genetype and T allele might be susceptible factors.

3. haplotype 521T - 10631 A might be the heredity risk factor of the development of cerebral infarction.

\section{LB-P17/93 \\ A Cross Sectional Survey to Determine the Impact of Educational Level on Awareness of Stroke}

\author{
Kamran S. ${ }^{1}$, Benner A.B. ${ }^{2}$ \\ ${ }^{1}$ Hamad General Hospital, Neurology, Doha, Qatar, \\ ${ }^{2}$ Hamad General Hospital, Doha, Qatar
}

Background: Stroke recognition is decisive in reducing delays to treatment. The aim of study was to determine the level of stroke awareness and risk factors among the middle aged population of Qatar.

Methods: A Prospective cross-sectional study involving primary health care centers where 1050 subjects were randomly approached for interview. Questionnaire included age, gender, marital status, education, occupation, income, knowledge of stroke (recognition, risk factors, revention, family history of 
stroke, common source of knowledge). Univariate and multivariate analyses were performed to identify variables independently associated with better interpretation of stroke symptoms.

Results: The response was 73\%, 767 (55.4\%) recognized the term stroke. Those who recognized the term stroke majority were $<40$ years. Awareness of stroke was seen mostly amongst the educated and it increased with level of education. Majority of the retired $(16.5 \%)$, housewives $(27.0 \%)$ and manual workers $(12.9 \%)$ were not aware of stroke. The clerical staff (39.5) and professional workers (11.5) were more likely to be aware. Those with hypertension $(25.1 \%)$, diabetics $(21.6 \%)$ and hypercholesteremia $(25.1 \%)$ were not aware of the term stroke. Commonly recognized symptoms were weakness, speech disorder, decreased sensation and decreased vision. Majority of the individuals who recognized stroke had obtained information from doctors and nurses $(79.1 \%)$.

Conclusion: This study highlights the need for a comprehensive stroke awareness program in Qatar to increase the awareness on the risk factors as well as recognizing the early signs and symptoms of stroke.

\section{LB-P17/95 \\ Hypertension and Risk of Stroke in Novosibirsk Population (HAPIEE Project)}

Simonova G., Shishkin S., Malyutina S., Skurihina Y., Nikitin Y.

Institute of Internal Medicine SB RAMS, Novosibirsk, Russian Federation

Purpose: To evaluate association between hypertension, isolated systolic hypertension, high pulse pressure and risk of stroke in prospective cohort 45-69 years old.

Methods and materials: Purpose population - 300000 citizens (Novosibirsk, Russia). Criteria of hypertension and isolated systolic hypertension were according to European recommendations (ESH, 2007). Stroke diagnosis was performed according WHO criteria. High pulse pressure was determined by difference between systolic and diastolic blood pressure $>60$. Total number of subjects: 7802 .

Results: Prevalence of hypertension in cohort during the primary screening was $59.4 \%$; isolated systolic hypertension $-10.1 \%$; high pulse pressure - $28.4 \%$.Prevalence of hypertension, isolated systolic hypertension, high pulse pressure in men and women was 27.8 and $31.5 \%, 12.3$ and $16.9 \%, 4.6$ and $5.6 \%$ respectively. Age adjusted average systolic BP in hypertensive men was 157.9 and $160.3 \mathrm{~mm}$ in hypertensive $(\mathrm{p}<0.001)$; diastolic BP - 98.6 and $98.7 \mathrm{~mm}$ respectively. Age adjusted stroke morbidity in cohort was 1.76 and 1.0 per 1000 man/years in men and women respectively. Stroke RR was 1.5 with hypertension, 2.1 with isolated systolic hypertension and 1.4 with high pulse pressure. Stroke PR for men/women correlation was 1.5, 2.2, 1.4 with hypertension, isolated systolic hypertension, high pulse pressure respectively. Average levels of total cholesterol, HDL cholesterol and triglycerides were 230.6 and $250.2 \mathrm{mmol} / \mathrm{l} ; 58.2$ and $60.6 \mathrm{mmol} / \mathrm{l} ; 130.3$ and $138.5 \mathrm{mmol} / \mathrm{l}$ in men and women respectively $(\mathrm{p}<0.001)$.
Conclusion: With high prevalence of hypertension and higher average levels of systolic BP, total cholesterol and triglycerides in women, men have higher risk of stroke.

\section{LB-P17/96 \\ Stroke Emergency Management: The Experience in Northern Lombardy, Italy \\ Vidale S. ${ }^{1}$, Longoni M. ${ }^{2}$, Gerardi F. ${ }^{3}$, Arnaboldi M. ${ }^{1}$, Guidotti M. ${ }^{4}$, Bezzi G. ${ }^{5}$, Bono G. ${ }^{6}$, Grampa G. ${ }^{7}$, Porazzi D. ${ }^{8}$, Zarcone D. ${ }^{9}$, Massei R. ${ }^{10}$, Landriscina M. ${ }^{11}$, Beghi E. ${ }^{3}$, Agostoni E. 12, on behalf of Northern Lombardia Emergenc y Stroke Study Group \\ 'Sant'Anna Hospital, Neurology Department, Como, Italy, ${ }^{2}$ Manzoni Hospital, Neurology Department, Lecco, Italy, ${ }^{3}$ Mario Negri Institute, Milan, Italy, ${ }^{4}$ Valduce Hospital, Neurology Department, Como, Italy, ${ }^{5}$ Valtellina Valchiavenna Hospital, Neurology Department, Sondrio, Italy, ${ }^{6}$ University of Insubria, Neurology Department, Varese, Italy, ${ }^{7}$ Circolo Hospital, Neurology Department, Saronno, Italy, ${ }^{8}$ Circolo Hospital, Neurology Department, Busto Arsizio, Italy, ${ }^{9} \mathrm{~S}$ Antonio Abate Hospital, Neurology Department, Gallarate, Italy, ${ }^{10}$ Manzoni Hospital, Emergency Department, Lecco, Italy, ${ }^{11}$ Sant'Anna Hospital, Emergency Department, Como, Italy, \\ ${ }^{12}$ Università degli Studi Bicocca- Milano, Department of \\ Neurosciences- Ospedale di Lecco, Lecco, Italy}

Background: The prognosis of stroke depends on the timing of medical interventions. Thrombolytic therapy is indicated when the patient is seen within 3 hours from the onset of symptoms. However, in clinical practice, in up to $80 \%$ of cases this time limit is exceeded.

Aims: To calculate the time spent during pre- and in-hospital steps in an advanced model of stroke care management in Northern Lombardy (total population: 2.100.000).

Methods: All the patients with stroke (WHO criteria) admitted to one of the 17 hospitals of the study area during a 4-months period were registered along with the time elapsed from the start of symptoms until the completion of the diagnostic work-up.

Results: 572 patients (mean age $73 \pm 12$ years; male/female ratio $1.2 / 1$ ). The mean (SD) time spent from symptom onset to ER admission was $236 \pm 291$ minutes (pre hospital time): $116 \pm$ 179 minutes for the arrival of the ambulance and $31 \pm 17$ minutes to reach the nearest hospital. The in- hospital time was 171 \pm 141 minutes: $81 \pm 130$ minutes to obtain a CT scan and $141 \pm$ 172 minutes for neurological consultation. The overall time spent varied across hospitals and favored patients using Emergency Services $(\mathrm{p}<0.001)$.

Conclusions: The time elapsed from the onset of stroke to the completion of diagnostic work-up varies across Italian hospitals and can be largely attributed to underuse of Emergency Services. These results prompt educational projects about stroke awareness and use of Emergency Services. 


\section{LB-P17/100}

\section{Looking into Stroke in Patients with a Clinical Diagnosis of Dementia: A Regional Population Based Study [Mapo Dementia Center]}

Han S.J., Yang D.W., Cho A.H.

Catholic University of Korea, Neurology, Seoul, Korea, Republic of Korea

Background: As two highly prevalent neurological diseases in the elderly, Alzheimer's disease and stroke were recently reported to have similar vascular risk factors in common. We aimed to investigate the prevalence of coexisting stroke and it's characteristics in patients with dementia.

Methods: We consecutively included patients referred from Mapo regional center for dementia in Seoul, Korea for work-up of dementia. The previous history of stroke was interviewed. Brain MRI, blood tests and risk factors were evaluated. The presence of lacunar infarcts, territorial or borderzone infarcts, previous hemorrhage, and leukoaraiosis were analyzed.

Results: Between February 2008 and March 2009, 80 patients were included. The most common type of dementia was Alzheimer's disease (AD) in 31(38\%), followed by vascular dementia in 21 and fronto-temporal dementia in 4. Seventeen $(21.3 \%)$ patients reported previous stroke history. However, MRI revealed that as much as $46(57.6 \%)$ patients had stroke lesion and $30(65.2 \%)$ of them had not recognized their stroke before this study. The stroke lesions were mostly lacunar infarcts in 41 patients and territorial infarcts or hemorrhage in 5. Old age and the presence of leukoaraiosis were associated with the presence of stroke lesion $(\mathrm{p}=0.012, \mathrm{p}=0.004)$. Even among 31patients with $\mathrm{AD}, 23(74.2 \%)$ patients had cerebral small vessel disease such as lacunar infarcts and leukoaraiosis.

Conclusions: About a half of patients who were screened as having dementia had stroke lesion on MRI.

Even in patients with definite diagnosis of degenerative dementia such as $\mathrm{AD}$, there was coexisting small vessel disease demanding further management for stroke prevention.

\section{LB-P17/101 \\ Circadian Variation in the Onset of Ischemic and Hemorrhagic Stroke}

Vidale S., Sacco L., Tancredi L., Arnaboldi M.

Sant'Anna Hospital, Neurology Department, Como, Italy

Background and purpose: While a circadian pattern of onset of acute myocardial infarction and sudden death has been observed in several studies, only few reports suggest that ischemic strokes (IS) occur more frequently in the early morning than in other times of the day. This variation is not observed for intracerebral hemorrhages (ICH). Aim of the study is to determine whether there is a circadian pattern of onset of the two main types of stroke.
Methods: A retrospective evaluation of patients hospitalised in the Stroke Unit of Como between 1 January 2006 and 31 December 2006 was conducted. Strokes were classified as IS and ICH. Diagnosis was confirmed by brain CT or MRI. Gender, date and time of onset were also registered. Time of onset was divided into three categories: morning ( 6 am to $12 \mathrm{pm}$ ), afternoon (12 pm to $8 \mathrm{pm}$ ) and night ( $8 \mathrm{pm}$ to $6 \mathrm{am})$. Statistical analysis was performed using the $\mathrm{chi}^{2}$ test.

Results: 340 patients were admitted for stroke. Men were $178(52.4 \%)$. IS was present in 282 cases $(82.9 \%)$ and ICH in $58(17.1 \%)$. Most strokes occurred during the morning $(58.8 \%)$ and only $9.4 \%$ in the night. While IS occurred mostly during the morning (196 pts), ICH peaked in the afternoon (42 pts) (p < 0.01 ). No associations were observed between week days of onset and stroke type.

Conclusion: These results confirm circadian trend of cerebrovascular disease onset and a distribution in the occurrence of ischemic and hemorrhagic strokes at different times.

\section{LB-P17/105 \\ Stroke Morbidity and Mortality in Asian Part of Russia}

Nikitin Y., Simonova G., Feigin V., Vinogradova T., Shishkin S., Skurihina Y., Pilipenko P.

Institute of Internal Medicine SB RAMS, Novosibirsk, Russian Federation

Purpose: To study stroke morbidity and mortality in Siberia and the Far East.

Methods: Stroke register in Novosibirsk 1982-2006 years (WHO program). Official census of enumeration.

Results: Stroke morbidity and mortality of Novosibirsk citizens 25-74 years old is registered since 1982, and also comparing with CVD risk factor prevalence registered on periodical screenings of the representative sample. Among men stroke morbidity increased during 25 years from 380 to 470 cases for 100000 citizens, in women - from 300 to 370 cases. Average annual trends of stroke morbidity and mortality were $1.1 \%$ in men and $1.2 \%$ in women. In Siberian and the Far East stroke morbidity vary from 206 to 450 cases for 100000 citizens, stroke mortality - from 74 to 200 cases (all ages, both gender). This indexes increase from West to East. Cerebral and ischemic stroke ratio changes in the same direction. Among some aboriginal ethnicities of Siberian North stroke is registered more rarely than among Caucasoids. We also studied prevalence of hypertension and other CVD risk factors in Siberia. These results were compared with stroke and CVD mortality. 


\section{Traumatic Brain Injury}

\section{P18/89 \\ Prospective Outcomes of Injury Study (POIS): Provisional Results from Phase One of a Qualitative Study}

\author{
Butler M., Derrett S. \\ Injury Prevention Research Unit, Dunedin School of \\ Medicine, Dunedin, New Zealand
}

Injuries are costly to New Zealand individuals and society. We currently know little about injured people's outcomes. The Prospective Outcomes of Injury Study (POIS) is a multi-method study that aims to: 1) Determine the injury, health and social rehabilitation, personal, social and economic factors leading to disability outcomes after injury for $2500 \mathrm{New}$ Zealanders (quantitative); and 2) Explore with 20 participants the lived experiences and perceptions of injury and outcomes (qualitative) in two interviews six months apart. This paper reports the initial results from the first phase of the qualitative component (Aim 2).

An interpretive phenomenological approach has been used to understand the meaning of injury in the context of a) work, family and community life and b) the macro-social environment created by a no-fault compensation scheme in New Zealand. Analysis has demonstrated a wide range of outcomes in terms of disability and biographical disruption following injury. The results fall into three groups: those who have a significant injury without any sense of biographical disruption, which includes those who have a prior neurological condition; those who have used the injury as a platform to improve their lives; and a small number of those for whom life has deteriorated since the injury. The latter group is primarily made up of those who have had a brain injury and young males. Findings have potential significance in elucidating ways that relative youth compounds the effect of brain injury by offering comparisons with other injuries.

P18/90

\section{Helmet Type and Nonstandard Use on Head Injuries in Motorcyclists}

\author{
Lin M.-R. ${ }^{1}, Y u$ W. $-Y^{2}$ \\ ${ }^{1}$ Institute of Injury Prevention and Control, Taipei Medical \\ University, Taipei, Taiwan, Republic of China, ${ }^{2}$ Department \\ of Emergency, Taipei Medical University Hospital, Taipei, \\ Taiwan, Republic of China
}

Background: The effectiveness of different helmet types in the prevention of head injuries may be considerably different, and protection of some helmet types as well as nonstandard helmet use may not be adequate.
Methods: A case-control study examined the effect of different helmet types (i.e. full-face, full- coverage, and partialcoverage) and non-standard helmet use on head injury. Logistic regression analysis was used to determine the roles of helmet type and non-standard use in the risk of head injury.

Results: During an 8-month period, 271 injured motorcyclists who visited the emergency room of a university hospital and were diagnosed to have a head injury were included as cases, and 271 who visited the same emergency room and had no head injury as controls. After adjustment potential confounders, compared with the full-face helmet, the full-coverage (odds ratio $(\mathrm{OR})=1.2$; 95\% confidence interval (CI): 0.7-2.2), partial-coverage helmet $(\mathrm{OR}=3.2 ; 95 \% \mathrm{CI}: 1.7-6.3)$, and unhelmeted $(\mathrm{OR}=7.2 ; 95 \% \mathrm{CI}$ : 2.7-19.3) increased the risk for a greater level of head injury the latter two results were statistically significant. Non-standard helmet use didn't significantly increase the risk of head injury.

Conclusions: The risk of head injury among motorcyclists was significantly increased by wearing a partial-coverage helmet when compared with the full-face helmet. In addition, it is unexpected that non-standard helmet use did not increase the risk of head injury among motorcyclists. Motorcycle riders are recommended to use full-face helmets only.

\section{P19 \\ Other Neurological Disorders}

\section{P19/91 \\ Epidemiological Research of Huntington Disease in Belarus}

Likhachev S., Pleshko I.

National Neurology and Neurosurgery Research Center, Minsk, Belarus

Background: It is very important to study the epidemiology of the Huntington disease (HD) disease in order to organize up-todate diagnostics, medical and social aid to the patients and their families.

Methods: Data over the last four years on 97 HD patients was studied retrospectively. The data was achieved through information dissemination to medical institutions of the country about $77(86.6 \%)$ patients, $20(13.4 \%)$ patients were treated in the hospital of the Centre.

Results: The prevalence of HD in the Republic of Belarus constituted 1 case per 100000 population. Out of 97 patients $72(74.2 \%)$ reside in cities, $25(25.8 \%)$ reside in rural regions. Women prevail among all patients $(53.6 \%)$, the ratio with male patients constituted 1.3:1. The disease onset in age under 20 was detected in 1 case (a female patient, the disease is manifested as choreic hyperkinesia).

Out of $20 \mathrm{HD}$ patients who were treated in the hospital of the Centre, there were 9 men, 11 women aged from 22 to 63 (average age $45 \pm 12.0$ ). In 19 patients the disease onset was manifested

Neuroepidemiology 2009;33:131-223 217 
through choreic hyperkinesia, in 1 case (a man, 26) the akineticorigid syndrome with athetoid movements in hands was detected. Intellectual-mnestic impairment was found in all patients. In 7 families out of $20 \mathrm{HD}$ was detected in 3 and more relatives in 2 generations.

Conclusion: Epidemiological research of the disease will make it possible to organize timely medical and social aid to HD patients and their families as well as medical and genetic consulting.

\section{LB-P19/94 \\ Urinary Tract Infection: Most Common Complications in Patients with Spinal Cord Injury \\ Milicevic S., Babovic R. \\ Clinic of Rehabilitation Dr M. Zotovic, Spinal Cord Injury, Belgrade, Serbia}

Introduction: injury to the spinal cord can lead to dysfunction of the urinary system, which in turn leads to an increased risk for the development of conditions such as urinary tract infection (UTI) and stones, vesicoureteral reflux, bladder diverticula, increased urinary residue, urinary obstruction and renal failure.

Methods: A retrospective study of 230 patients with spinal cord injury admitted to the Clinic of rehabilitation Dr M. Zotovic in Belgrade, Serbia from January 2000 to December 2004. A total of 250 patients were reviewed: 162 paraplegic (43 women and 119 men) and 88 tetraplegic (16 women and 72 men). Complete laboratory tests, including urine culture and antibiogram were performed for all patients.

Results: The most frequent complications were urinary infection $(78,4 \%)$, spasticity $(55,6 \%)$, pressure ulcers $(2,9 \%)$, pneumonia $(1,9 \%)$, renal calculi $(1,4 \%)$, autonomic dysreflexia $(0,9 \%)$, deep venous thrombosis (2\%). Escherichia coli (24,3\%), Proteus mirabilis (21\%), Pseudomonas aerug. (18,4\%) were the most common findings. Escherichia coli was the most common organism resistant to routine drugs. Maximum resistance existed towards Amoxicillin (87,4\%), Cephalexin (72,3\%), and Trimetoprim sulphometoxasol $(54,2 \%)$ and least resistance towards Imipenem (3\%) and Gentamicin (24,4\%).

Conclusion: Urinary tract infections (UTIs) in the SCI population frequently are asymptomatic, polymicrobial, caused by antibiotic-resistant bacteria, and very likely to recur or relapse. However, there are no data to support or justify treatment of asymptomatic bacteriuria.

\section{LB-P19/103 \\ Epidemiology of Prion Diseases in Humans in Uruguay}

\begin{abstract}
Perna A. ${ }^{1}$, Lewin S. ${ }^{2}$, Salinas D. ${ }^{3}$, Rodriguez M.M. ${ }^{4}$, Vernengo L. ${ }^{4}$, Mañana G. ${ }^{5}$, Ketzoian C. ${ }^{1}$, Salamano R. ${ }^{2}$

${ }^{1}$ Institute of Neurology, Neuroepidemiology Section., Montevideo, Uruguay, ${ }^{2}$ Institute of Neurology,

Neuroinfectology Section, Montevideo, Uruguay, ${ }^{3}$ Institut of Neurology, Montevideo, Uruguay, ${ }^{4}$ Faculty of Medicine, Genetic Department, Montevideo, Uruguay, ${ }^{5}$ Hospital of Clinics, Neuropathology Department, Montevideo, Uruguay
\end{abstract}

Prion diseases are degenerative diseases of the central nervous system, which invariably lead to death. Their course is progressive over months and they have long periods without clinical manifestations. The aim of this retroprospective study was to perform the survey of diagnosed prion diseases in Uruguay between 1984 and 2009. In total, 38 cases of Creutzfeldt - Jacob disease (CDJ) were identified (6 possible, 27 probable and 5 definitive cases); 7 of them corresponded to the hereditary form and 31 to the sporadic form. The estimated incidence rate from 1997 to date was 0.68 cases per million inhabitants per year. The clinical features of the disease were similar to those reported in the literature. So far no single case of the new variant of Creutzfeldt - Jacob disease (nvCJD) or other types of human prion diseases have been reported in the Uruguayan population. Uruguay has one hundred neurologists distributed all over the country who are members of the Uruguayan Neurologists Society; therefore it is easy to collect almost all the cases and submit a good and updated survey to the authorities of the Public Health Ministry.

\section{LB-P19/104 \\ Functional Impairment is Associated with Increased Mortality in the NEDICES Cohort Study}

Vega-Quiroga S. ${ }^{1}$, Benito-León J. ${ }^{2}$, Bermejo-Pareja F.', Vega Valderrama L.M. ${ }^{3}$, Pinlla Sanchez J.M. ${ }^{4}$,

Valderrama Valderrama M.L. ${ }^{5}$, Trincado R. ${ }^{6}$, Medrano M.J. ${ }^{7}$, Boix R. ${ }^{7}$, Villarejo A. ${ }^{6}$, Rodriguez $C^{8}{ }^{8}$

${ }^{1}$ Health Center/ SACYL, Primary Care, El Espinar, Spain, 'University Hospital '12 de Octubre',, Department of Neurology, CIBERNED, Madrid, Spain, ${ }^{3}$ Clinic Hospital, Internal Medicine, Valladolid, Spain, ${ }^{4} \mathrm{SACYL}$, Primary Care Administration, Segovia, Spain, ${ }^{5}$ Health Center/SACYL, Primary Care, Nava de la Asunción, Spain, ${ }^{6}$ University Hospital '12 de Octubre', Department of Neurology, Madrid, Spain, ${ }^{7}$ Institute Carlos III, Epidemiology National Centre, Madrid, Spain, ${ }^{8}$ University Hospital '12 de Octubre', CIBERNED, Madrid, Spain

Objective: To evaluate the mortality, ten years after the baseline wave, of participants with functional impairment from 
the Neurological Disorders in Central Spain (NEDICES) Cohort Study, a population-based cohort study in the elderly ( $\geq 65$ years).

Methods: A prospective, population-based design was used to compare the risk of mortality in participants with functional impairment (those subjects with a Pfeffer scale score $\geq 6$ points) vs. participants without functional impairment in three communities in central Spain (NEDICES). We obtained mortality data 10 years later (2004) of the baseline evaluation (1994 to 1995). The relative risk (RR) of mortality (participants with functional impairment vs. controls) was estimated using Cox proportional hazards models that excluded participants with dementia or questionable dementia.
Results: There were 210 (56.5\%) deaths among 372 participants with functional impairment and 884 (25.7\%) among 3,442 participants without functional impairment. In a Cox model that adjusted for baseline age, gender, educational category, and comorbidity, risk of mortality was increased in participants with functional impairment $(\mathrm{RR}=1.86 ; 95 \% \mathrm{CI}=1.58-2.20$; $\mathrm{p}=0.001)$. Using the Kaplan-Meier method, mean survival was 7.03 years $(95 \% \mathrm{CI}=6.64-7.42)$ for participants with functional impairment and 9.33 years $(95 \% \mathrm{CI}=9.24-9.42)$ for participants with normal function.

Conclusions: In this longitudinal, prospective study, functional impairment was independently associated with 10-year mortality among community dwelling older persons, even after adjusting for other risk factors. 
Abdulwahab, A. 184

Abe, E. 203

Abholz, H.-H. 171

Aboyans, V. 173

Adeleye, A. 184

Adeoye, O. 151

Agarwal, D. 176

Aggelopoulou, C. 212

Agostoni, E. 215

Aitken, R. 150, 164

Akova-Öztürk, E. 211, 212

Akshulakov, S. 143

Alamanos, Y. 179

Albers, K. 161

Albert, A. 181

Ali, H.M. 176

Alvarenga, M. 149

Alvarenga, M.P. 200

Alvarenga, R. 154

Alvarenga, R.M.P. 200

Alwell, K. 151

Amensi, A. 164

Aminisani, N. 175

Anand, A. 156

Andelic, N. 142

Andersen, K.K. 135, 151

Antequera, D. 185

Appelros, P. 134, 150

Arami, M.A. 207

Aridon, P. 137, 167, 173

Arnaboldi, M. 215, 216

Artzoglou, V. 179

Ashtari, F. 141

Asplund, K. 134, 150

Ausin Villaverde, V. 137

Avakyan, G. 205

Awaad, Y. 138, 156, 188

Azarpazhooh, M.R. 169

Ažman, D. 167

Baasanjav, D. 203

Babovic, R. 218

Bajdik, C. 195

Bakhtazad, A. 166

Balaguer-Martinez, E. 204

Balata, A. 188

Baldin, E. 140

Bandzouzi, B. 173

Banerjee, T.K. 148

Bankl, H.-C. 165

Barakova, A. 175

Barker-Collo, S. 135, 201

Barreira, A. 149

Bar-Shira, A. 157

Bartko, D. 163, 175

Basic-Kes, V. 168, 191
Beghi, E. 147, 153, 160, 176, 215

Bejot, Y. 145

Bell, G.S. 148, 153

Benedetti, M.D. 140, 197, 198, 199

Benito, J. 137

Benito-León, J. 154, 155, 182 , 184, 185, 209, 218

Benner, A.B. 214

Benuskova, L. 178

Beran, R. 136, 152

Bermejo-Pareja, F. 154, 155, 182, 184, 185, 209, 218

Bernard-Bourzeix, L. 172

Bernard, M. 141

Bezdolniy, Y. 205

Bezzi, G. 215

Bickel, H. 171, 180, 181

Birbilis, T. 212

Biserni, P. 153

Björk, J. 134

Blair, A. 137

Blanco-Centurion, C. 169

Blazicek, P. 163, 175

Boix, R. 154, 182, 184, 209, 218

Bokor, M. 205

Bonissoni, S. 199

Bono, G. 215

Borhani Haghighi, A. 196, 206

Boström, I. 195

Bottacchi, E. 209

Bougioukou, A. 186

Boyd, L. 163

Boysen, G. 167

Brady, J. 139

Brainin, M. 145

Brandis, A. 179

Breitenfeld, T. 168

Briesenick, C. 180

Broderick, J. 151

Broenner, M. 180, 181

Brown, A. 141

Brüggemann, N. 204

Brum, D. 149

Bruno, E. 200

Budišić, M. 193, 194

Buhmann, C. 204

Butler, M. 217

Cabral, N. 146

Callander, M. 195

Callari, G. 137

Camargo, S. 149
Campos Arillo, V. 204

Cardwell, C. 177

Carmona, O. 140, 194

Carod Artal, F.J. 138, 205

Carro, E. 185

Carwell, C. 178

Catberro, A. 160

Caula, J. 140

Chahal N. 201

Chandra, S. 176

Charnukha, T. 206

Chaudhuri, A. 148

Chen, C.-P. 192

Chen, H. 137

Chen, L. 190

Chen, Q. 190

Chiang, C.-E. 210

Chiappedi, M. 153

Chiò, A. 160

Cho, A.H. 216

Christe, W. 174

Cilia, S. 200

Ciudad, R. 194

Clément, J.P. 172, 173, 183

Colic, G. 185

Collimedaglia, L. 199

Combor, I. 163, 175

Consoli, D. 147

Cooray, M. 164

Cordato, D. 136, 152, 164

Cordero Guevara, J. 137

Correnti, A. 213

Corso, G. 209

Cossu, P. 188, 198

Couratier, P. 160, 161, 172

Crespi, V. 147

Cubo, E. 137

Cvitanović Šojat, L. 191

Dahl, O.-P. 196

Dalbem, J. 154

D'Alessandro, R. 140, 147

D'Amelio, M. 137, 167, 173

Danisova, J. 163

Dantoine, T. 172

da Silveira Ribeiro, L. 138

Das, S.K. 148

Dastgiri, S. 175

Daumer, M. 139

Dechamps, N. 172

De Deyn, P.P. 181

Degenhardt, A. 139

Dehghan, A. 206

Delaj, L. 140, 147

De Lepeleire, J. 181

Delgado Benito, V. 137
Delgado, I. 158

Demarin, V. 143, 167, 168, 191, 193, 194

Dent, W. 192, 202

De Reuck, J. 174

Derrett, S. 217

Desport, J.C. 161

Diaz- Guzman, J. 209

Díaz, L. 140

Diaz, V. 158

Di Benedetto, N. 137, 167

Diener, H.-C. 144

Di Giovanni, M. 209

Ding, D. 211

Ding, X. 170, 171, 208

Dispenza, S. 213

Djientcheu, V.D.P. 159, 162, 165

Dobi, D. 176

Doi, Y. 206

Dommes, P. 144

Dong, Z. 189

Drude, L. 204

Duan, J. 214

Duggan, M. 139

Durier, J. 145

Eatemadi, M.M. 169

Ebers, G. 139

Eckhardt, R. 145

Edwards, J. 163

Eluf-Neto, J. 146

Epstein, D. 136

Erälinna, J.-P. 200

Eriksson, M. 150

Escobar, A. 162

Esiene, A. 165

Essono Mba, R. 159

Etgen, T. 180

Evers, S. 211, 212

Faiss, J. 174

Famoso, G. 137, 167

Fang, Y. 192

Faragó, A. 205

Farzadfard, M.T. 169

Feichtinger, J. 165

Feigin, V. 135, 201, 216

Ferioli, S. 151

Ferrara, A. 161

Ferrari-Ginevra, O. 153

Ferrari, J. 145

Ferst, P. 146

Filippini, G. 197

Fitzpatrick, A. 177, 178

Fitzpatrick, P. 139

\section{KARGER}

(C) 2009 S. Karger AG, Basel

Fax +41613061234

E-Mail karger@karger.ch

www.karger.com

Accessible online at:

www.karger.com/ned 
Flaherty, M. 151

Foerstl, H. 180, 181

Fonseca, L.A.M., 146

Forment, J.C. 140

Förster, A. 146

Fučić, A. 191

Fu, J.-H. 211

Fujimura, M. 187

Gajofatto, A. 198, 199

Galán, J.M. 137

Galeotti, M. 140

Gandolfo, C. 147

Gao, Y. 158

García-Ramos, R. 185

Gattellari, M. 150, 164

Gerardi, F. 215

German, I. 201

German, L. 201

Ghaem, H. 196, 206

Ghandehary, K. 169

Ghayour-Mobarhan, M. 169

Ghezzo, A. 153

Ghia, D. 136, 152

Giacone, S. 160

Giannikopoulos, G. 199

Giannoulos, I. 199

Giardini, G. 209

Gigineishvili, D. 152, 187

Giladi, N. 157

Giroud, M. 145

Gjergja Juraški, R. 191

Golabi, M. 170

Goldbourt, U. 172

Goldstein, J. 177

Golubovic, S. 185, 186

Gomes Camargo, S.M.G. 200

Gonçalves, A. 146

Gonzalez-Hervias, L. 183

Goto, Y.-I. 186

Gousias, K. 179

Grampa, G. 215

Grandas-Perez, F. 204

Granella, F. 140

Greco, G. 147

Grønbæk, M. 167

Gruber-Mösenbacher, U. 165

Guekht, A. 168

Güell, E. 194

Guerchet, M. 173

Guidolin, L. 140

Guidotti, M. 215

Gulyaeva, N. 168

Gundersen, M.D. 160

Gusev, E. 168, 205

Gutiérrez-Álvarez, A.M. 189

Hackembruch, H. 158

Hackl, M. 165

Hagenah, J. 204

Hainfellner, J.A. 165

Hajat, S. 213

Han, S.J. 216

Hao, Z. 207
Harner, N. 139

Hashemilar, M. 175

Hashimoto, S. 195

Hassanpour, H. 167

Hauser, W.A. 168

Hazra, A. 148

Heinzl, H. 165

He, J.L. 211, 214

Heliopoulos, I. 212

Hennerici, M. 146

Hernández Ramos, F.J. 184

Hirose, K. 147, 210

Hodgkinson, S. 136, 152

Hofer, C. 145

Hollenbeck, A. 137

Hong, J. 189

Hong, Z. 135, 211

Houinato, D. 173

Huang, X. 137

Hulter Asberg, K. 134, 150

Hussein, K. 179

Hussein, S. 179

Hutchinson, M. 139

$\mathrm{Hu}, \mathrm{Y} .171$

Hylkema, M. 189

Hyson, M. 191

Ichikawa, M. 147, 210

Idriceanu, C. 165

Invernizzi, P. 198

Ivanković, M. 143

Ivanoiu, A. 181

Ivanovic, N. 156

Ivashynka, A. 153, 180

Ivičević Bakuli, T. 191

Izumi, M. 157, 208

Jacquin, A. 145

Jadue, L. 158

Jakobsson, K. 134

Jalaludin, B. 150, 164

Janculjak, D. 143

Janghorbani, M. 141

Jasse, L. 141

Jaw, F.-S. 192

Jemea, B. 159

Jena, S. 148

Ji, X. 190

Jongen, P.J.H. 149

Jonsson, F. 134, 150

Jordan, S. 139

Jung, K.-Y. 188

Kaaya, J. 136, 155, 192, 202

Kahana, C. 201

Kajeu, P.-J. 161

Kamga, Y. 165

Kamran, S. 214

Kapisyzi, M. 176

Karamouzos, E. 199

Kasabov, N. 171, 178

Kassubek, J. 136, 155

Kasten, M. 204

Katsarava, Z. 144
Katunina, E. 205

Kern, R. 146

Kerschbaumsteiner, K. 192, 202

Kertelge, L. 204

Ketzoian, C.N. 158, 218

Khaimovsky, I. 168

Khan, M. 176

Khatri, P. 151

Khayat Nouri, M.H. 170

Khoury, J. 151

Khullar, M. 156

Kiani, R. 169

Kiefer, A. 165

Kim, J.M. 182

Kim, S.W. 182

Kingwell, E. 195

Kinsella, K. 139

Kisic Tepavcevic, D. 156

Kissela, B. 151

Kita, Y. 147, 210

Klein, C. 204

Kleindorfer, D. 151

Knežević-Pavlić, M. 193

Kobayashi, M. 203

Kobela, M. 165

Kong, F. 207

König, H.-H. 183

Koopmans, P. 149

Kostic, V. 156

Kountouris, D. 186

Kovacik, M. 163, 175

Kowa, H. 208

Kreipe, H. 179

Kruja, J. 176

Kuchtova, Z. 175

Kulatunga, A. 164

Kummer, W. 138

Kuqo, A. 176

Kurtis, M. 183

Kyriakides, T. 166

Kyritsis, A. 179

Lai, C.-H. 202

Laisa, P. 200

La Mantia, L. 197

Landriscina, M. 215

Landtblom, A.-M. 195

Lang, W. 145

Lauer, K. 195, 198

Lavados, P. 158, 162

Lawes, C. 135

Lebedeva, A. 168

Lechowicz, W. 173

Lee, I.K. 188

Lee, S.-Y. 188

Leibson, C. 141

Leijten, F. 189

Lei, X. 171

Lekoubou Looti, A.Z. 159, 162, 165

Leone, M.A. 140, 153, 199

Leoni, S. 188, 198

Levy, A. 163

Lewin, S. 218
Liang, L. 171

Li, D. 159

Lietava, J. 175

Likhachev, S. 180, 206, 217

Likhterman, L. 143

Lin, L. 190

Lin, M.-R. 217

Lin, S. 207

Li, S. 148,158

Li, S.C. 135

Liu, H.M. 159

Liu, M. 145, 169, 177, 207, 214

Li, X. 192

Li, Z. 158

Lo Fermo, S. 200

Logroscino, G. 160

Lohmann, K. 204

Lonergan, R. 139

Long, N. 189

Longo, A. 146

Longoni, M. 215

Lorenzano, S. 213

Louis, E.D. 137, 155

Lovrenčić-Huzjan, A. 143, 193, 194

Luck, T. 171, 183

Lu, C.-Z. 211

Lugaresi, A. 140

Luis Marott, J. 167

Luppa, M. 183

Lydersen, S. 196

Macarrón Vicente, J. 137

Mccarron, P. 177, 178

Mcconville, J. 177, 178

Mc Kenna, M. 139

Madarasz, S. 163, 175

Madjidova, Y. 213

Maggioni, E. 153

Maier, H. 165

Maier, W. 171

Maimone, D. 200

Majdi, M.R. 169

Makaya, M. 164

Malec, J. 141

Malyutina, S. 215

Mamiya, S. 203

Mañana, G. 218

Manca, S. 198

Mandrekar, J. 141

Manfredi, M. 147

Marin, B. 160, 161

Markou, M. 179

Martinez-Martin, P. 138, 183, 204, 205

Martínez, R. 194

Martín Santidrián, A. 137

Massacesi, L. 197

Massei, R. 215

Masuet, C. 140

Matschinger, H. 183

Mattana, F. 147, 153

Matuja, W.B.P. 192 
Mazzola, M.A. 167, 173

M'Belesso, P. 173

Mecarelli, O. 213

Medrano, M. 209

Medrano, M.J. 154, 155, 182, 218

Meehan, S. 163

Meindl, M. 136, 155, 192, 202

Mendez-Robles, N. 183

Messina, S. 200

Mets, T. 181

Meza-Rojas, G. 205

Michelli, F. 205

Midgard, R. 160, 196

Mignon, A. 181

Mikula, I. 194

Milanese, C. 197

Milicevic, S. 218

Millul, A. 160, 176

Milz, R.U. 211, 212

Miškov, S. 191

Miura, K. 147, 210

Mokhber, N. 169

Moldovanu, I. 144

Molina, J.A. 185

Monaco, F. 199

Montero, C. 140

Moomaw, C. 151

Morales, J.M. 154

Morando, G. 198

Moreno, E. 194

Moreno, T. 185

Morita, Y. 147, 210

Moro, C. 146

Motti, L. 140

Motz, R. 165

Mouanga, A.M. 173

Mu, L. 189

Müller, D. 144

Muraro, P. 139

Murray, B. 139

Musolino, R. 147

Mygland, A. 142

Nagata, K. 157, 208

Nagy, Z. 210

Nakagawa, E. 186

Nakamura, Y. 147, 210

Nakashima, K. 208

Nakayasu, H. 208

Naldi, P. 199

Namvaran, A. 170

Ndoumbe, A. 159

Neligan, A. 148, 153

Nepomnyashy, V. 143

Neri, W. 140

Neuhaus, A. 139

Nghemmogne Takam, C. 162

Nguefack, S. 165

Nicholas, R. 139

Nicoletti, A. 200

Nikitin, Y. 215, 216

Njamnshi, A.K. 165

Njamnshi, K.A. 159, 162
Nkam, M. 159

Norrving, B. 134, 150

Nubukpo, P. 172, 173, 183

Obara, K. 203

Obermann, M. 144

Obiakor, O.R. 184

Ogboi, J.S. 184

Oger, J. 195

Ohnishi, H. 203

Oikonen, M. 200

Okayama, A. 147, 210

Olazarán, J. 182

Oliveira, F. 146

Olsen, T.S. 135, 151

Orr-Urtreger, A. 157

Ortiz Salas, P. 189

Osa-Ruiz, E. 183

Osseby, G.-V. 145

Osuna, T. 194

Oudin, A. 134

Óváry, C. 210

Pablo, M.J. 194

Palau, D. 189

Pals, P. 181

Panahandeh, M. 169

Papacostas, S. 166

Papaioakim, M. 212

Papais-Alvarenga, M. 149, 200

Papais-Alvarenga, R.M. 149

Parag, V. 135

Paraïso, M.N. 173

Park, Y. 137

Pasedag, T. 179

Pathmeswaran, A. 164

Patti, F. 200

Pavelin, S. 143

Paz, M. 140, 194

Pechanova, O. 175

Pekmezovic, T. 156

Pélisson, D. 141

Peretz, C. 157

Perkal, H. 140

Perla, F. 199

Perna, A. 158, 218

Peters, A. 213

Peters, G. 149

Petrik, O. 163

Petrovic, I. 156

Phillips, N. 195

Phillips, T. 172

Pilipenko, P. 216

Pinlla Sanchez, J.M. 218

Pinzone, F. 167

Piperidou, C. 212

Piras, C. 141

Platel, M. 178

Plavec, D. 143, 193

Pleshko, I. 217

Polyzoidis, K. 179

Porazzi, D. 215

Posada, I.J. 155

Prabhakar, S. 156
Prado-Jean, A. 172

Prencipe, M. 213

Preusser, M. 165

Preux, P.M. 160, 161, 173, 183

Pribisynscki, V. 183

Prieto-Jurcynska, C. 183

Prina, L. 162

Provinciali, L. 147

Puca, E. 213

Puertas-Martin, V. 182, 209

Puertas, V. 185

Pugliatti, M. 188, 198

Pupillo, E. 160, 176

Quattrocchi, G. 200

Quesenberry, C. 161

Quintero Cusguen, P. 189

Radon, K. 213

Ragonese, P. 137, 167, 173

Ramió-Torrentà, L. 140

Ramirez Moreno, J.M. 184

Ranawaka, U.K. 164

Ransom, J. 141

Rasulova, K. 213

Razmkon, A. 166

Reggiani, M. 209

Reiner-Concin, A. 165

Ren, L. 192

Richling, B. 165

Rieckmann, P. 197

Riedel-Heller, S.G. 171, 183

Riise, T. 188

Ringelstein, E.-B. 211, 212

Roberto, M. 200

Rodriguez-Blazquez, C. 205

Rodríguez, C. 154, 155, 182, 218

Rodriguez, M.M. 218

Rodriguez Rodriguez, C. 209

Rohalova, J. 175

Rosati, G. 188, 198

Rossi, F. 198, 199

Rössler, K. 165

Rosso, M.G. 199

Rouaud, O. 145

Roy, B. 148

Roy, T. 148

Ruggerone, S. 199

Ruiz-Galeano, G. 205

Rumana, N. 147, 210

Rushkevich, Y. 206

Ryglewicz, D. 173

Sabitu, K. 184

Sacco, L. 216

Sacks, C. 162

Saez Velasco, S. 137

Salamano, R. 218

Salemi, G. 173

Salinas, D. 218

Salinas, R. 162

Sanchez-Ferro, A. 184

Sander, D. 180,181
Sander, J.W. 148, 153

Sanders, E. 149

Sandhaug, M. 142

Santangelo, M. 140, 147

Santos, G.A.C. 200

Sarno, C. 167

Savadi Oskuei, D. 175

Savettieri, G. 137, 167, 173

Savoj, M.R. 141

Scalfari, A. 139

Scarpatetti, M. 165

Schatzkin, A. 137

Schelosky, L. 174

Schenkel, J. 213

Schliebs, S. 178

Schmidt, A. 204

Schmutzhard, E. 136, 155, 192, 202

Schneider, S.A. 204

Schwartz, R. 172

Scott, J. 174

Sedivy, R. 165

Seemueller, S. 139

Seiler, S. 142

Serdari, A. 212

Serrano-Dueñas, M. 205

Shahar, D.R. 201

Shakarishvili, R. 152

Shamshirgaran, S.M. 175

Shan, J. 161

Shaygannejad, V. 141

Shen, X. 190

Shimamoto, T. 187

Shin, I.S. 182

Shiromani, P. 169

Shishkin, S. 215, 216

Shorvon, S.D. 148,153

Siebner, H. 204

Simonova, G. 215, 216

Siqueira, H. 154

Skurihina, Y. 215, 216

Sobolev, B. 163

Solari, A. 197

Song, B. 158

Sotgiu, S. 198

Specchio, L. 147

Spelta, P. 153

Stamoulis, I. 199

Stawiarz, L. 195

Stefanini, M.C. 153

Stegmayr, B. 134, 150

Stelzhammer, B. 192, 202

Stiglbauer, W. 165

Stinear, C. 163

Stordal, E. 196

Strehlau, V. 197

Strineka, M. 193

Stroh, E. 134

Strömberg, U. 134

Sugawara, M. 203

Sugihara, H. 147, 210

Sun, H.Y. 211, 214

Supanc, V. 168

Surana, A. 176 
Suto, Y. 208

Suzuki, K. 157, 208

Svetel, M. 156

Szabó, G. 210

Szabo, K. 146

Tadic, V. 204

Taghipour, M. 166

Takahashi, K. 206

Takashima, N. 147, 210

Tancredi, L. 216

Tang, J. 170

Tango, T. 206

Tanne, D. 172

Tan, S. 158

Tao, W. 207

Tapias, E. 185

Tatschl, C. 145

Teal, P. 163

Terént, A. 134, 150

Terruso, V. 137, 167

Tetsuya, M. 164

Thomas, P. 136, 152

Thrift, A.G. 169

Thuler, L.C.S. 200

Tilikete, C. 141

Tissera, N. 164

Tola, M.R. 140

Tonali, P.A. 153

Toni, D. 213

Tonini, C. 153

Toniolo, M. 198

Tossi, C. 162

Toyoshima, I. 203

Trejo, G. 137

Tremlett, H. 195, 197

Trendafilova, A. 136, 155

Trincado, R. 154, 155, 182, 184, 185, 209, 218
Tripodaki, E. 199

Truelsen, T. 167

Tseng, H.-F.T. 202

Tsivgoulis, G. 212

Tsouni, P. 199

Tubridy, N. 139

Tumpić-Jaković, J. 193

Turatti, M. 198, 199

Turin, T. 147, 210

Tütüncü, E. 136, 155

Ueshima, H. 147, 210

Uijl, S. 189

Vadikolias, K. 212

Valderrama Valderrama, M.L. 218

van den Bussche, H. 171

Van Den Eeden, S.K. 161

van der Tol, M. 149

van der Vegt, J. 204

Vandewoude, M. 181

Van Maele, G. 174

van Noort, E. 149

Vardanian, C. 141

Vargas, T. 185

Vargek-Solter, V. 168

Varona, F. 184

Vasconcelos, C. 149

Vasconcelos, C.C.F. 200

Vassanelli, C. 198

Vatne, A. 142

Vecchio, D. 199

Vedovello, M. 198, 199

Vega-Quiroga, S. 218

Vega, S. 154, 182, 184, 185, 209

Vega Valderrama, L.M. 218

Velazquez, C. 205
Velez van Meerbeke, A. 189

Ventura, M. 181

Vernengo, L. 218

Ververaki, S. 199

Vidale, S. 215, 216

Vieira, C. 146

Vighetto, A. 141

Vignatelli, L. 140

Villarejo, A. 155, 182, 185, 209, 218

Vinish, M. 156

Vinogradova, T. 216

Virues-Ortega, J. 205

Visser, L. 149

Voulgaris, S. 179

Vuković, V. 143, 167, 191, 193, 194

Vukusic, S. 141

Waldhör, T. 165

Wang, D. 177, 207

Wang, M. 190

Wang, W. 148

Wang, W.Z. 135, 159

Wang, X. 192

Wang, Z. 190

Wassenaar M. 189

Weinstein, G. 172

Weyerer, S. 171

Wickramaratna, C. 164

Wickramasinghe, R. 164

Winkler, A. 136, 155, 192, 202

Wisse, C. 204

Wissel, J. 174

Wöhrer, A. 165

Wolf, M. 146

Woo, D. 151

Worthington, J. 136, 150, 152, 164
Wreksoatmodjo, B. 142, 208

Wu, B. 145, 207

Wu, J. 148

Wu, L. 190

Wu, L.E. 211

Xu, Q. 137

$\mathrm{Xu}, \mathrm{Y} .158$

Ya, E. 203

Yang, C. 189

Yang, D.W. 216

Yang, G.A. 211, 214

Yang, Q.D. 135

Yang, W. 177, 207

Yaseen, R. 160

Yazdchi, M. 207

Ydreos, I. 199

Yie, S.-D. 188

Yin, Z. 192

Yokoyama, T. 206

Yoon, J.S. 182

Yoon, M.-S. 144

Yu, W.-Y. 217

Yuhua, Z. 189

Zappia, M. 200

Zarcone, D. 215

Zavoreo, I. 168

Zeighami, B. 206

Zerbi, D. 176

Zhang, A. 192

Zhang, R. 158

Zhao, L. 158

Zoccolella, S. 160

Zorzou, M.-P. 199

Zou, Z. 190

Zwanikken, C. 149 